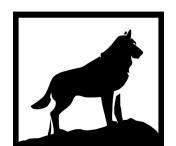

Michigan

Technological

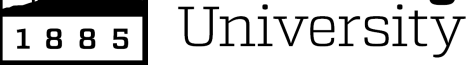

Michigan Technological University

Digital Commons @ Michigan Tech

\title{
AMBIENT NOISE SEISMIC INTERFEROMETRY THROUGH SURFACE SEISMIC DATA ACQUISITION VS ZERO-OFFSET VERTICAL SEISMIC PROFILING (VSP)
}

Erdi Apatay

Michigan Technological University, eapatay@mtu.edu

Copyright 2020 Erdi Apatay

Recommended Citation

Apatay, Erdi, "AMBIENT NOISE SEISMIC INTERFEROMETRY THROUGH SURFACE SEISMIC DATA ACQUISITION VS ZERO-OFFSET VERTICAL SEISMIC PROFILING (VSP)", Open Access Master's Thesis, Michigan Technological University, 2020.

https://doi.org/10.37099/mtu.dc.etdr/1113

Follow this and additional works at: https://digitalcommons.mtu.edu/etdr

Part of the Geological Engineering Commons 
AMBIENT NOISE SEISMIC INTERFEROMETRY THROUGH SURFACE SEISMIC DATA ACQUISITION VS ZERO-OFFSET VERTICAL SEISMIC PROFILING (VSP)

\title{
By
}

Erdi Apatay

\author{
A THESIS \\ Submitted in partial fulfillment of the requirements for the degree of \\ MASTER OF SCIENCE \\ In Geophysics
}

MICHIGAN TECHNOLOGICAL UNIVERSITY

2020

(C) 2020 Erdi Apatay 
This thesis has been approved in partial fulfillment of the requirements for the Degree of MASTER OF SCIENCE in Geophysics.

Department of Geological and Mining Engineering and Sciences

Thesis Advisor: Dr. Roohollah Askari

Committee Member: $\quad$ Dr. Gregory P. Waite

Committee Member: Dr. Nathan D. Manser

Department Chair: Dr. Aleksey Smirnov 


\section{Table of Contents}

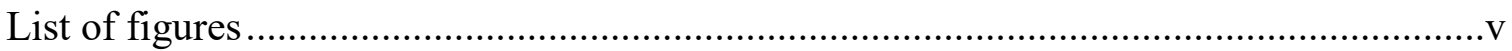

List of tables....................................................................................................... xix

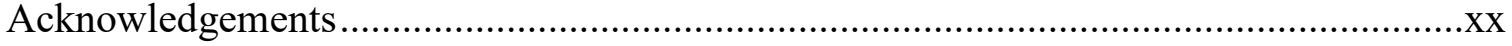

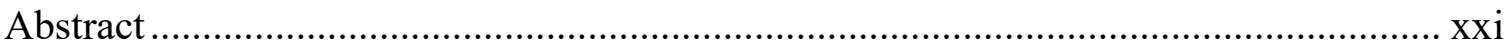

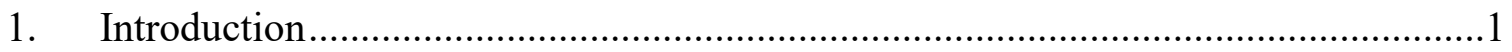

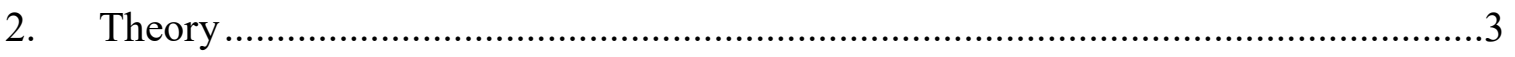

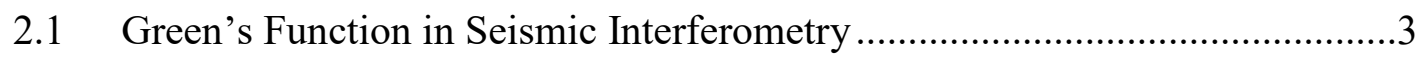

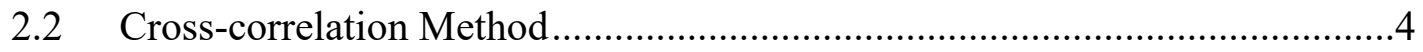

2.3 2D Finite-Difference Wavefield Modeling Software.......................................5

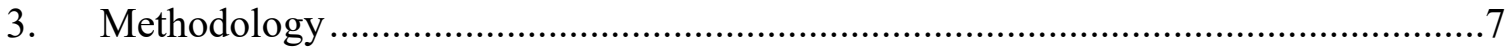

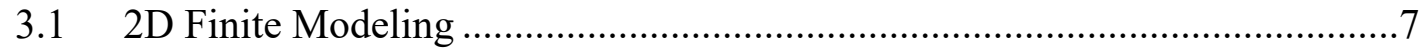

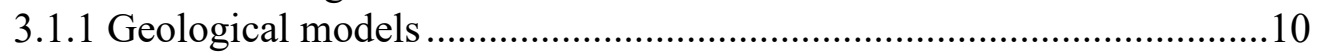

3.1.2 Surface data acquisition and processing ..............................................14

3.1.3 Zero-Offset VSP ..............................................................................15

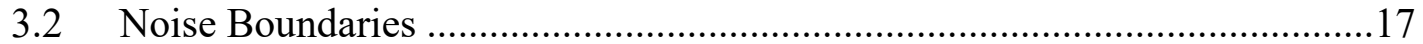

3.3 The Cross-correlation of Zero-Offset VSP and Seismic Interferometry........19

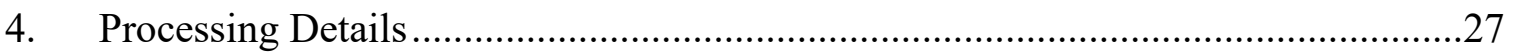

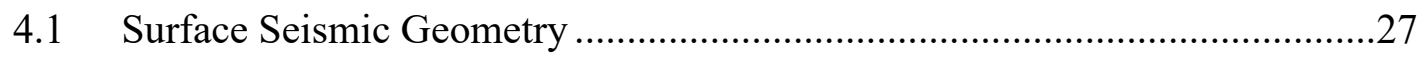

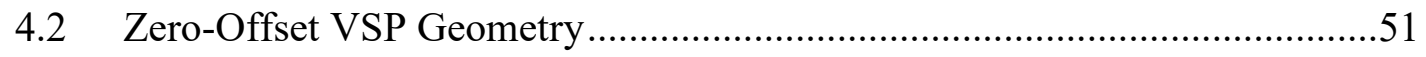

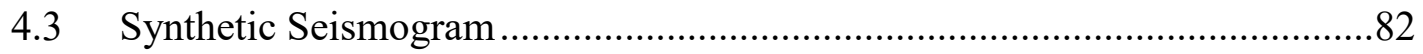




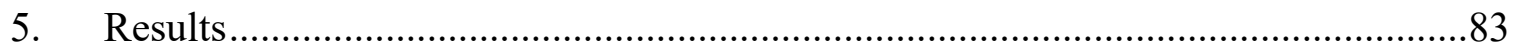

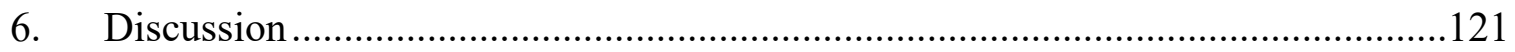

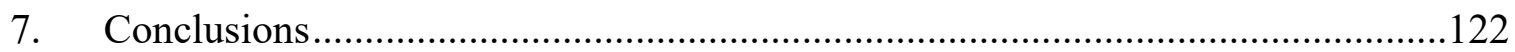

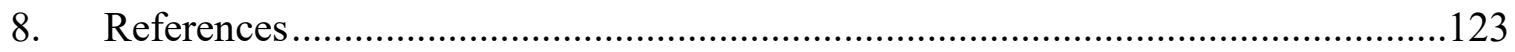

9. Appendix A) Zero-Offset VSP Cross-correlation MATLAB Code ......................128

10. Appendix B) Zero-Offset VSP Processing on Seismic Unix...............................129

11. Appendix C) Generating Synthetic Seismogram MATLAB Code.........................134 


\section{List of figures}

Figure 1. The fundamental principle of reflected-wave interferometry (Schuster, 2001, 2009). (a) The subsurface source is emitted a signal from underground to the surface and is recorded by a geophone. (b) A second geophone receives the signal by an underground scatterer. (c) The cross-correlation cancels the common path from source to the first receiver.

Figure 2. (a) The 20 random source signatures with varying source duration. (b) One source signature. (c) The amplitude spectrum of the source signature in (b) ...................... 8

Figure 3. (a) shows the beginning and (b) shows the end of the signal amplitude presented

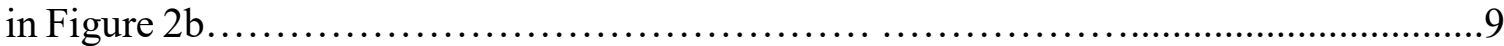

Figure 4. An acoustic velocity model with flat layers that are combined surface seismic and zero-offset VSP geometry. The green lateral line shows geophone array for surface seismic geometry. Blue triangles (V) correspond to 201 geophones that are placed on the free surface. L3 (covered by yellow-dashed area) corresponds to the coal layer. The geophones are layered on the free surface between $0 \mathrm{~m}$ to $10,000 \mathrm{~m}$. The vertical orange line shows VSP line, the red triangles ( $(101$ geophones) show the array of the geophones, which are layered between $1,000 \mathrm{~m}$ and $3,000 \mathrm{~m}$, used in the VSP geometry............... 11

Figure 5. An acoustic velocity model with anticlined layers that are combined surface seismic and zero-offset VSP geometry. The green lateral line shows geophone array for surface seismic geometry. Blue triangles (V) correspond to 201 geophones that are placed on the free surface. L3 (covered by yellow-dashed area) corresponds to the coal layer. The geophones are layered on the free surface between $0 \mathrm{~m}$ to $10,000 \mathrm{~m}$. The vertical orange line shows VSP line, the red triangles (V) (101 geophones) show the array of the geophones, which are layered between $1,000 \mathrm{~m}$ and $3,000 \mathrm{~m}$, used in the VSP geometry...........12

Figure 6. An acoustic velocity model with 3 anticlines and 1synclines that are combined surface seismic and zero-offset VSP geometry. The green lateral line shows geophone array for surface seismic geometry. Blue triangles (V) correspond to 201 geophones that are placed on the free surface. L3 (covered by yellow-dashed area) corresponds to the coal layer. The geophones are layered on the free surface between $0 \mathrm{~m}$ to $10,000 \mathrm{~m}$. The vertical orange line shows VSP line, the red triangles (V) (101geophones) show the array of the geophones, which are layered between $1,000 \mathrm{~m}$ and $3,000 \mathrm{~m}$, used in the VSP

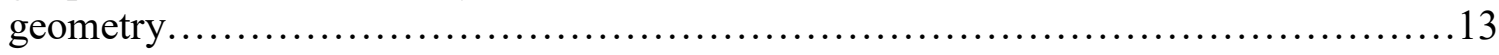

Figure 7 . The processing flow of the surface seismic interferometry................ 14

Figure 8 . The processing flow of the zero-offset VSP interferometry.................. 16

Figure 9. Four different noise boundaries for surface seismic geometry and zero-offset VSP geometry used in this study. The lateral horizontal dark purple line on the surface shows 
the surface seismic geometry. The vertical thick yellow line shows the VSP tool positioned $(5,000 \mathrm{~m}, 3,000 \mathrm{~m})$. The noise boundary 1 (vertical red dashed lines) laterally extends from $4,500 \mathrm{~m}$ to $5,500 \mathrm{~m}$; the noise boundary 2 (vertical green dashed lines) laterally extends from $4,000 \mathrm{~m}$ to $6,000 \mathrm{~m}$; the noise boundary 3 (vertical blue dashed lines) laterally extends from $2,000 \mathrm{~m}$ to $8,000 \mathrm{~m}$, and the noise boundary 4 (vertical orange dashed lines)

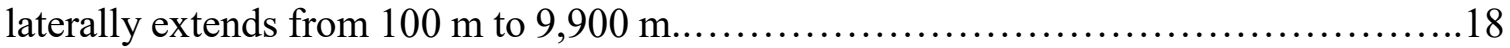

Figure 10. The surface seismic cross-correlation procedure in seismic interferometry. ( ) represents the geophone, while $\left({ }_{n}\right)$ corresponds to the source. $\mathrm{N}$ denotes the last geophone.

Figure 11. (a) The $120 \mathrm{~s}$ long acoustic transmission response (noisy data). (b) The master trace 101 among all 201 traces. (c) The reconstructed reflection response or virtual shot gather as if from a source at 5,000 $\mathrm{m}$

Figure 12. The cross-correlation procedure for the zero-offset VSP interferometry. This figure was generated utilizing Matsushima (2016). .23

Figure 13. The retrieving VSP reflection response from noisy VSP data by crosscorrelation method. (a) shows uncorrelated noisy VSP data. (b) shows the first trace used in the cross-correlation as a master trace. (c) shows the virtual VSP shot gather after crosscorrelation method .26

Figure 14. A raw data of 120 seconds long of boundary 1 for 20 sources. The noise sources starting from $100 \mathrm{~m}$ to 3,900 $\mathrm{m}$ depth and extend from 4,500 $\mathrm{m}-5,500 \mathrm{~m}$ lateral distance

Figure 15. The comparison of virtual shot gathers of 5 sources (left-hand side), 20 sources (right-hand side), and 500 sources (bottom) for boundary 1, 2, 3, and 4 in Figure 15a, Figure $15 \mathrm{~b}$, Figure 15c, and Figure 15d in the flat model, respectively. The yellow arrows

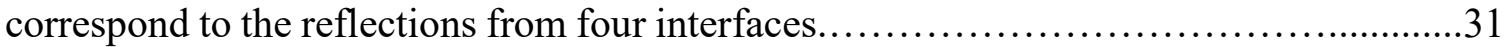

Figure 16. The comparison of virtual shot gathers of 5 sources (left-hand side), 20 sources (right-hand side), and 500 sources (bottom) for boundary 1, 2, 3, and 4 in Figure 16a, Figure $16 \mathrm{~b}$, Figure 16c, and Figure 16d in the anticline model, respectively. The yellow arrows correspond to the reflections from four interfaces. .35

Figure 17. The comparison of virtual shot gathers of 5 sources (left-hand side), 20 sources (right-hand side), and 500 sources (bottom) for boundary 1, 2, 3, and 4 in Figure 17a, Figure $17 \mathrm{~b}$, Figure 17c, and Figure 17d in the anticline - syncline model, respectively. The yellow arrows correspond to the reflections from four interfaces.............................

Figure 18. (a) Before velocity picking of CMP gather 2000 for 500 sources boundary 1. (b) After velocity picking of CMP gather 2000 for 500 sources boundary 1 in the flat model as an example. 
Figure 19. (a), (b), (c) and (d) shows the unmigrated zero-offset sections obtained from boundaries 1, 2, 3, and 4 for 5 sources about the flat model,

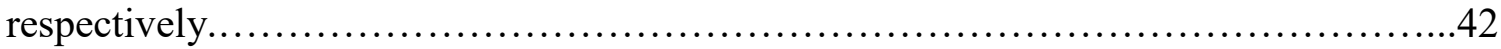

Figure 20. (a), (b), (c) and (d) shows the unmigrated zero-offset sections obtained from boundaries 1, 2, 3, and 4 for 20 sources about the flat model,

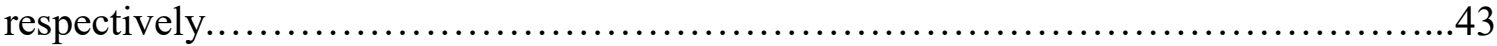

Figure 21. (a), (b), (c) and (d) shows the unmigrated zero-offset sections obtained from boundaries 1, 2, 3, and 4 for 500 sources about the flat model,

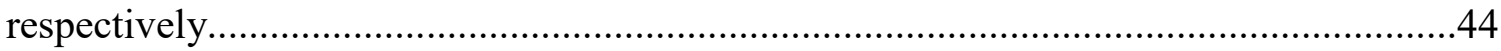

Figure 22. (a), (b), (c) and (d) shows the unmigrated zero-offset sections obtained from boundaries $1,2,3$, and 4 for 5 sources about the anticline model, respectively.

Figure 23. (a), (b), (c) and (d) shows the unmigrated zero-offset sections obtained from boundaries 1, 2, 3, and 4 for 20 sources about the anticline model, respectively

Figure 24. (a), (b), (c) and (d) shows the unmigrated zero-offset sections obtained from boundaries 1, 2, 3, and 4 for 500 sources about the anticline model, respectively.

Figure 25. (a), (b), (c) and (d) shows the unmigrated zero-offset sections obtained from boundaries 1, 2, 3, and 4 for 5 sources about the anticline - syncline model, respectively.

Figure 26. (a), (b), (c) and (d) shows the unmigrated zero-offset sections obtained from boundaries 1, 2, 3, and 4 for 20 sources about the anticline - syncline model, respectively.

Figure 27. (a), (b), (c) and (d) shows the unmigrated zero-offset sections obtained from boundaries 1, 2, 3, and 4 for 500 sources about the anticline - syncline model, respectively.

Figure 28. The noisy VSP data for the flat model using 20 randomly distributed sources in boundary 1 as an example. The horizontal axis shows the geophone number while the

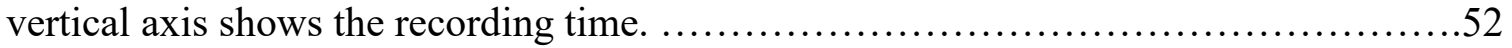

Figure 29. (a), (b), (c), and (d) show virtual VSP shot gather after cross-correlation for boundaries 1, 2, 3, and 4 for the flat model using 5 sources, respectively. The red arrow shows downgoing primary wave or first-break curve, the yellow arrows show downgoing multiples, and the blue arrows show upgoing waves. .54 
Figure 30. (a), (b), (c), and (d) show virtual VSP shot gather after cross-correlation for boundaries 1, 2, 3, and 4 for the flat model using 20 sources, respectively. The red arrow shows downgoing primary wave or first-break curve, the yellow arrows show downgoing multiples, and the blue arrows show upgoing waves.

Figure 31. (a), (b), (c), and (d) show virtual VSP shot gather after cross-correlation for boundaries 1, 2, 3, and 4 for the flat model using 500 sources, respectively. The red arrow shows downgoing primary wave or first-break curve, the yellow arrows show downgoing multiples, and the blue arrows show upgoing waves. .58

Figure 32. (a), (b), (c), and (d) show virtual VSP shot gather after cross-correlation for boundaries 1, 2, 3, and 4 for the anticline model using 5 sources, respectively. The red arrow shows downgoing primary wave or first-break curve, the yellow arrows show downgoing multiples, and the blue arrows show upgoing waves.....................60

Figure 33. (a), (b), (c), and (d) show virtual VSP shot gather after cross-correlation for boundaries 1(a), (b), (c), and (d) show virtual VSP shot gather after cross-correlation for boundaries 1, 2, 3, and 4 for the anticline model using 20 sources, respectively. The red arrow shows downgoing primary wave or first-break curve, the yellow arrows show downgoing multiples, and the blue arrows show upgoing waves. .62

Figure 34. (a), (b), (c), and (d) show virtual VSP shot gather after cross-correlation for boundaries 1, 2, 3, and 4 for the anticline model using 500 sources, respectively. The red arrow shows downgoing primary wave or first-break curve, the yellow arrows show downgoing multiples, and the blue arrows show upgoing waves.....................64

Figure 35. (a), (b), (c), and (d) show virtual VSP shot gather after cross-correlation for boundaries 1, 2, 3, and 4 for the anticline-syncline model using 5 sources, respectively. The red arrow shows downgoing primary wave or first-break curve, the yellow arrows show downgoing multiples, and the blue arrows show upgoing waves.

Figure 36. (a), (b), (c), and (d) show virtual VSP shot gather after cross-correlation for boundaries 1, 2, 3, and 4 for the anticline-syncline model using 20 sources, respectively. The red arrow shows downgoing primary wave or first-break curve, the yellow arrows show downgoing multiples, and the blue arrows show upgoing waves. ................68

Figure 37. (a), (b), (c), and (d) show virtual VSP shot gather after cross-correlation for boundaries 1, 2, 3, and 4 for the anticline-syncline model using 500 sources, respectively. The red arrow shows downgoing primary wave or first-break curve, the yellow arrows show downgoing multiples, and the blue arrows show upgoing waves.

Figure 38. (a) shows the VSP data containing 20 sources for boundary 1 in the flat model. The red line shows downgoing primary, the yellow arrows show downgoing multiples, and the blue arrows show upgoing waves or reflections. (b) shows the rectangular section closer. This section was chosen as an example in terms of showing the central points of 
maximum energy (red dashes) picked on the traces between the 21 st and 31 st traces. $\mathrm{X}$-axis

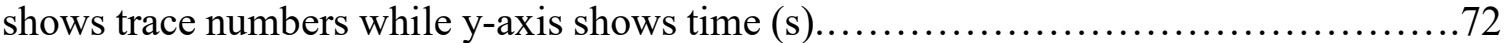

Figure 39. The VSP data obtained by adding geophone depth values in the borehole. The red line shows the picked traces in Figure 38a. The red line shows downgoing primary wave, the yellow arrows show downgoing multiples, and the blue arrows show upgoing waves.

Figure 40. Separation of the downgoing wavefield and the upgoing wavefield. (a) shows before frequency-wavenumber filtering. (b) shows the downgoing wavefield positioned in the positive quadrant. (c) shows the upgoing wavefield positioned in the negative quadrant. The data that was used in the fdelmodc for VSP was $24 \mathrm{~Hz}$, which can be seen in the Figure. $\mathrm{x}$-axis shows wavenumber $(1 / \mathrm{m})$ while the $\mathrm{y}$-axis $(\mathrm{Hz})$ shows frequency.................74

Figure 41. The separated upgoing wavefield. The red line shows the P-wave downgoing

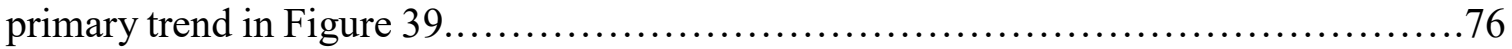

Figure 42. The separated downgoing wavefield. The red line shows the P-wave downgoing

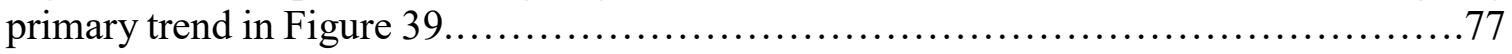

Figure 43. The flattened upgoing wavefield. The red narrow corridor corresponds to the outside corridor stack, containing 10 traces. The receivers on the VSP in the borehole are

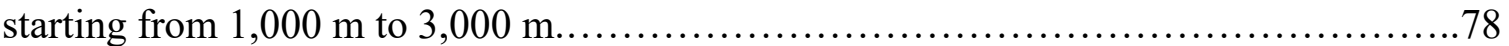

Figure 44. The corridor stack that consists of 10 traces corresponding to the red narrow

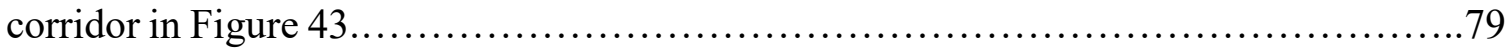

Figure 45. The combined surface seismic and zero-offset VSP data. The yellow arrows show the reflections from interfaces in the surface seismic flat model. The rectangular area in the middle corresponds to the 10 traces of the zero-offset VSP positioned offset between $5,025 \mathrm{~m}$ and $5,250 \mathrm{~m}$ on the surface.

Figure 46. The wiggle Figure of splicing the corridor stack into the surface seismic between $5,025 \mathrm{~m}$ and 5,250 $\mathrm{m}$ offset.

Figure 47. The illustration of comparison of the seismic, the VSP, and the synthetic seismograms of a flat model created using 5 sources for boundary 1. (a), (b), (c), and (d) show the retrieved stacked section, the VSP seismogram, the seismic seismogram, and the synthetic seismogram, respectively. In (c), the 2 nd red rectangular box shows traces corresponding to the reflection from coal layer. The yellow arrow shows the interface of the coal layer. The coal layer has been detected by the seismic method. The 1st reflection (the 1st red rectangular boxes in (b) and (c)) has been detected by the VSP method and the seismic method together. The 3rd reflection (the 3rd red rectangular box in (c)) has been detected by the seismic method. The 4th reflection has not been detected by both methods .85 
Figure 48. The illustration of comparison of the seismic, the VSP, and the synthetic seismograms of a flat model created using 5 sources for boundary 2. (a), (b), (c), and (d) show the retrieved stacked section, the VSP seismogram, the seismic seismogram, and the synthetic seismogram, respectively. In (c), the 2nd red rectangular box shows traces corresponding to the reflection from coal layer. The yellow arrow shows the interface of the coal layer. The coal layer has been detected by the seismic method. The 1 st reflection (the 1st red rectangular boxes in (b) and (c)) has been detected by the VSP method and the seismic method together. The 3rd reflection (the 3rd red rectangular box in (c)) has been detected by the seismic method. The 4th reflection has not been detected by both

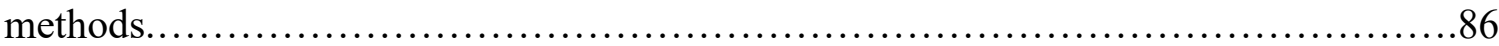

Figure 49. The illustration of comparison of the seismic, the VSP, and the synthetic seismograms of a flat model created using 5 sources for boundary 3. (a), (b), (c), and (d) show the retrieved stacked section, the VSP seismogram, the seismic seismogram, and the synthetic seismogram, respectively. In (c), the 2 nd red rectangular box shows traces corresponding to the reflection from coal layer. The yellow arrow shows the interface of the coal layer. The coal layer has been detected by the seismic method. The 1 st reflection (the 1st red rectangular boxes in (b) and (c)) has been detected by the VSP method and the seismic method together. The 3rd reflection (the 3rd red rectangular box in (c)) has been detected by the seismic method. The 4th reflection has not been detected by both methods

Figure 50. The illustration of comparison of the seismic, the VSP, and the synthetic seismograms of a flat model created using 5 sources for boundary 4. (a), (b), (c), and (d) show the retrieved stacked section, the VSP seismogram, the seismic seismogram, and the synthetic seismogram, respectively. In (c), the 2 nd red rectangular box shows traces corresponding to the reflection from coal layer. The yellow arrow shows the interface of the coal layer. The coal layer has been detected by the seismic method. The 1 st reflection (the 1st red rectangular boxes in (b) and (c)) has been detected by the VSP method and the seismic method together. The 3rd reflection (the 3rd red rectangular box in (c)) has been detected by the seismic method. The 4th reflection has not been detected by both methods .88

Figure 51. The illustration of comparison of the seismic, the VSP, and the synthetic seismograms of a flat model created using 20 sources for boundary 1. (a), (b), (c), and (d) show the retrieved stacked section, the VSP seismogram, the seismic seismogram, and the synthetic seismogram, respectively. In (c), the 2 nd red rectangular box shows traces corresponding to the reflection from coal layer. The yellow arrow shows the interface of the coal layer. The coal layer has been detected by the seismic method. The 1 st reflection (the 1st red rectangular boxes in (b) and (c)) has been detected by the VSP method and the seismic method together. The 3rd reflection (the 3rd red rectangular box in (c)) has been detected by the seismic. The 4th reflection has been detected by the seismic method (the 
4th red rectangular box in (c)), but the VSP method has been unsuccessful to detect this reflection.

Figure 52. The illustration of comparison of the seismic, the VSP, and the synthetic seismograms of a flat model created using 20 sources for boundary 2. (a), (b), (c), and (d) show the retrieved stacked section, the VSP seismogram, the seismic seismogram, and the synthetic seismogram, respectively. In (c), the 2 nd red rectangular box shows traces corresponding to the reflection from coal layer. The yellow arrow shows the interface of the coal layer. The coal layer has been detected by the seismic method. The 1st reflection (the 1st red rectangular boxes in (b) and (c)) has been detected by the VSP method and the seismic method together. The 3rd reflection (the 3rd red rectangular box in (c)) has been detected by the seismic method. The 4th reflection has been detected by the seismic method (the 4th red rectangular box in (c)), but the VSP method has been unsuccessful to detect this reflection. .90

Figure 53. The illustration of comparison of the seismic, the VSP, and the synthetic seismograms of a flat model created using 20 sources for boundary 3. (a), (b), (c), and (d) show the retrieved stacked section, the VSP seismogram, the seismic seismogram, and the synthetic seismogram, respectively. In (c), the 2 nd red rectangular box shows traces corresponding to the reflection from coal layer. The yellow arrow shows the interface of the coal layer. The coal layer has been detected by the seismic method. The 1 st reflection (the 1st red rectangular boxes in (b) and (c)) has been detected by the VSP method and the seismic method together. The 3rd reflection (the 3rd red rectangular box in (c)) has been detected by the seismic method. The 4th reflection has been detected by the seismic (the 4th red rectangular box in (c)), but the VSP method has been unsuccessful to detect this reflection.

Figure 54. The illustration of comparison of the seismic, the VSP, and the synthetic seismograms of a flat model created using 20 sources for boundary 4. (a), (b), (c), and (d) show the retrieved stacked section, the VSP seismogram, the seismic seismogram, and the synthetic seismogram, respectively. In (c), the 2 nd red rectangular box shows traces corresponding to the reflection from coal layer. The yellow arrow shows the interface of the coal layer. The coal layer has been detected by the seismic method. The 1 st reflection (the 1st red rectangular boxes in (b) and (c)) has been detected by the VSP method and the seismic method together. The 3rd reflection (the 3rd red rectangular box in (c)) has been detected by the seismic method. The 4th reflection has been detected by the seismic method (the 4th red rectangular box in (c)), but the VSP method has been unsuccessful to detect this reflection.

Figure 55. The illustration of comparison of the seismic, the VSP, and the synthetic seismograms of a flat model created using 500 sources for boundary 1. (a), (b), (c), and (d) show the retrieved stacked section, the VSP seismogram, the seismic seismogram, and the synthetic seismogram, respectively. In (c), the 2 nd red rectangular box shows traces 
corresponding to the reflection from coal layer. The yellow arrow shows the interface of the coal layer. The coal layer has been detected by the seismic method. The 1st reflection (the 1st red rectangular boxes in (b) and (c)) has been detected by the VSP method and the seismic method together. The 3rd reflection (the 3rd red rectangular box in (c)) has been detected by the seismic method. The 4 th reflection has been detected by the seismic method (the red rectangular box in (c)), but the VSP method has been unsuccessful to detect this reflection .93

Figure 56. The illustration of comparison of the seismic, the VSP, and the synthetic seismograms of a flat model created using 500 sources for boundary 2. (a), (b), (c), and (d) show the retrieved stacked section, the VSP seismogram, the seismic seismogram, and the synthetic seismogram, respectively. In (c), the 2nd red rectangular box shows traces corresponding to the reflection from coal layer. The yellow arrow shows the interface of the coal layer. The coal layer has been detected by the seismic method. The 1 st reflection (the 1st red rectangular boxes in (b) and (c)) has been detected by the VSP method and the seismic method together. The 3rd reflection (the 3rd red rectangular box in (c) has been detected by the seismic method. The 4th reflection has been detected by the seismic method (the red rectangular box in (c)), but the VSP method has been unsuccessful to detect this reflection.

Figure 57. The illustration of comparison of the seismic, the VSP, and the synthetic seismograms of a flat model created using 500 sources for boundary 3. (a), (b), (c), and (d) show the retrieved stacked section, the VSP seismogram, the seismic seismogram, and the synthetic seismogram, respectively. In (c), the 2 nd red rectangular box shows traces corresponding to the reflection from coal layer. The yellow arrow shows the interface of the coal layer. The coal layer has been detected by the seismic method. The 1st reflection (the 1st red rectangular boxes in (b) and (c)) has been detected by the VSP method and the seismic method together. The 3rd reflection (the 3rd red rectangular box in (c)) has been detected by the seismic method. The 4 th reflection has been detected by the seismic method (the 4th red rectangular box in (c)), but the VSP method has been unsuccessful to detect this reflection.

Figure 58. The illustration of comparison of the seismic, the VSP, and the synthetic seismograms of a flat model created using 500 sources for boundary 4. (a), (b), (c), and (d) show the retrieved stacked section, the VSP seismogram, the seismic seismogram, and the synthetic seismogram, respectively. In (c), the 2nd red rectangular box shows traces corresponding to the reflection from coal layer. The yellow arrow shows the interface of the coal layer. The coal layer has been detected by the seismic method. The 1st reflection (the 1st red rectangular boxes in (b) and (c)) has been detected by the VSP method and the seismic method together. The 3rd reflection (the 3rd red rectangular box in (c) has been detected by the seismic method. The 4 th reflection has been detected by the seismic method 
(in (c)), but the VSP method has been unsuccessful to detect this reflection.

Figure 59. The illustration of comparison of the seismic, the VSP, and the synthetic seismograms of an anticline model created using 5 sources for boundary 1. (a), (b), (c), and (d) show the retrieved stacked section, the VSP seismogram, the seismic seismogram, and the synthetic seismogram, respectively. In (b), the red rectangular box shows traces corresponding to the reflection from coal layer. The yellow arrow shows the climax of the interface of the coal layer. The coal layer has been detected by the VSP method. The 1st reflection (the 1st red rectangular box in (c)) has been detected by the seismic method. The 3 rd reflection (the 3rd red rectangular box in (c)) has been detected by the seismic method. The 4th reflection has not been detected by both methods

Figure 60. The illustration of comparison of the seismic, the VSP, and the synthetic seismograms of an anticline model created using 5 sources for boundary 2. (a), (b), (c), and (d) show the retrieved stacked section, the VSP seismogram, the seismic seismogram, and the synthetic seismogram, respectively. In (b), the red rectangular box shows traces corresponding to the reflection from coal layer. The yellow arrow shows the climax of the interface of the coal layer. The coal layer has been detected by the VSP method. The 1st reflection (the 1 st red rectangular box in (c)) has been detected by the seismic method. The 3 rd reflection (the 3rd red rectangular box in (c)) has been detected by the seismic method. The 4 th reflection has not been detected by both methods............................98

Figure 61. The illustration of comparison of the seismic, the VSP, and the synthetic seismograms of an anticline model created using 5 sources for boundary 3. (a), (b), (c), and (d) show the retrieved stacked section, the VSP seismogram, the seismic seismogram, and the synthetic seismogram, respectively. In (b), the red rectangular box shows traces corresponding to the reflection from coal layer. The yellow arrow shows the climax of the interface of the coal layer. The coal layer has been detected by the VSP method. The 1st reflection (the 1st red rectangular box in (c)) has been detected by the seismic method. The 3 rd reflection (the $3 \mathrm{rd}$ red rectangular box in (c) has been detected by the seismic method. The 4th reflection has not been detected by both methods.

Figure 62. The illustration of comparison of the seismic, the VSP, and the synthetic seismograms of an anticline model created using 5 sources for boundary 4. (a), (b), (c), and (d) show the retrieved stacked section, the VSP seismogram, the seismic seismogram, and the synthetic seismogram, respectively. The reflection from the coal layer has been detected by the seismic method (the 2 nd red box in (c)). The yellow arrow shows the climax of the interface of the coal layer. The 1st and the 3rd reflections have been detected by the seismic method. The 4th reflection has been detected by the VSP method (in (a)) and the seismic method (in (c)). 100 
Figure 63. The illustration of comparison of the seismic, the VSP, and the synthetic seismograms of an anticline model created using 20 sources for boundary 1. (a), (b), (c), and (d) show the retrieved stacked section, the VSP seismogram, the seismic seismogram, and the synthetic seismogram, respectively. The reflection from the coal layer has been detected by the VSP method only (the 2nd red box in (b)). The yellow arrow shows the climax of the interface of the coal layer. The 1st reflection has been detected by the seismic method (in (c) and by the VSP method (in (a)). The 3rd reflection has been detected by the seismic method (the 3rd red rectangular box in (c)). Both methods have been unsuccessful to detect the 4th reflection.

Figure 64. The illustration of comparison of the seismic, the VSP, and the synthetic seismograms of an anticline model created using 20 sources for boundary 2. (a), (b), (c), and (d) show the retrieved stacked section, the VSP seismogram, the seismic seismogram, and the synthetic seismogram, respectively. The reflection from the coal layer has been detected by the VSP method (the 2 nd red box in (b)). The yellow arrow shows the climax of the interface of the coal layer. The 1st reflection has been detected by both methods (in (b) and in (c)). The 3rd reflection has been detected by the seismic method (the 3rd red rectangular box in (c)). Both methods have been unsuccessful to detect the 4th reflection.

Figure 65. The illustration of comparison of the seismic, the VSP, and the synthetic seismograms of an anticline model created using 20 sources for boundary 3. (a), (b), (c), and (d) show the retrieved stacked section, the VSP seismogram, the seismic seismogram, and the synthetic seismogram, respectively. The reflection from the coal layer has been detected by the VSP method (the 2 nd red box in (b)). The yellow arrow shows the climax of the interface of the coal layer. The 1st reflection has been detected by both methods (in (b) and in (c)). The 3rd reflection has been detected by the seismic method (the 3rd red rectangular box in (c)). Both methods have been unsuccessful to detect the 4th reflection. 103

Figure 66. The illustration of comparison of the seismic, the VSP, and the synthetic seismograms of an anticline model created using 20 sources for boundary 4. (a), (b), (c), and (d) show the retrieved stacked section, the VSP seismogram, the seismic seismogram, and the synthetic seismogram, respectively. The reflection from the coal layer has been detected by the VSP method (the 2 nd red box in (b)). The yellow arrow shows the climax of the interface of the coal layer. The 1st reflection has been detected by both methods (in (b) and in (c)). The 3rd reflection has been detected by the seismic method (the 2nd red rectangular box in (c)). The 4th reflection has been detected by the VSP method (the 4th red rectangular box in $b)$ ). 104

Figure 67. The illustration of comparison of the seismic, the VSP, and the synthetic seismograms of an anticline model created using 500 sources for boundary 1. (a), (b), (c), and (d) show the retrieved stacked section, the VSP seismogram, the seismic seismogram, 
and the synthetic seismogram, respectively. The reflection from the coal layer has been detected by the VSP method (the 2nd red box in (b)). The yellow arrow shows the climax of the interface of the coal layer. The 1st reflection has been detected by both methods (in (b) and in (c)). The 3rd reflection has been detected by the seismic method (the red rectangular box in (c)). Both methods have been unsuccessful to detect the 4th reflection. 105

Figure 68. The illustration of comparison of the seismic, the VSP, and the synthetic seismograms of an anticline model created using 500 sources for boundary 2. (a), (b), (c), and (d) show the retrieved stacked section, the VSP seismogram, the seismic seismogram, and the synthetic seismogram, respectively. The reflection from the coal layer has been detected by the VSP method (the 2 nd red box in (b)). The yellow arrow shows the climax of the interface of the coal layer. The 1st reflection has been detected by both methods (in (b) and in (c)). The 3rd reflection has been detected by the seismic method (the 2nd red rectangular box in (c)). Both methods have been unsuccessful to detect the 4th reflection................................................................ 106

Figure 69. The illustration of comparison of the seismic, the VSP, and the synthetic seismograms of an anticline model created using 500 sources for boundary 3. (a), (b), (c), and (d) show the retrieved stacked section, the VSP seismogram, the seismic seismogram, and the synthetic seismogram, respectively. The reflection from the coal layer has been detected by the VSP method (the 2 nd red box in (b)). The yellow arrow shows the climax of the interface of the coal layer. The 1st reflection has been detected by both methods (in (b) and in (c)). The 3rd reflection has been detected by the seismic method (the 3rd red rectangular box in (c)). The 4th reflection has been detected by the seismic method (in (c))

Figure 70. The illustration of comparison of the seismic, the VSP, and the synthetic seismograms of an anticline model created using 500 sources for boundary 4. (a), (b), (c), and (d) show the retrieved stacked section, the VSP seismogram, the seismic seismogram, and the synthetic seismogram, respectively. The reflection from the coal layer has been detected by the VSP method (the 2 nd red box in (b)). The yellow arrow shows the climax of the interface of the coal layer. The 1st reflection has been detected by both methods (in (b) and in (c)). The 3rd reflection has been detected by the seismic method (the 3rd red rectangular box in (c)). The 4th reflection has been detected by the seismic method (in (c)) 108

Figure 71. The illustration of comparison of the seismic, the VSP, and the synthetic seismograms of an anticline-syncline model created using 5 sources for boundary 1. (a), (b), (c), and (d) show the retrieved stacked section, the VSP seismogram, the seismic seismogram, and the synthetic seismogram, respectively. The reflection from the coal layer has been detected by the seismic method only (the 2 nd red rectangular box in (c)). The yellow arrow shows the climax of the interface of the coal layer. The 1st reflection has 
been detected by the seismic method (the 1 st red rectangular box in (c)). The 3rd reflection has been detected by the VSP method (the red rectangular box in (b)). The 4th reflection has not been detected by both methods.

Figure 72. The illustration of comparison of the seismic, the VSP, and the synthetic seismograms of an anticline-syncline model created using 5 sources for boundary 2. (a), (b), (c), and (d) show the retrieved stacked section, the VSP seismogram, the seismic seismogram, and the synthetic seismogram, respectively. The reflection from the coal layer has been detected by the seismic method only (the 2nd red rectangular box in (c)). The yellow arrow shows the climax of the interface of the coal layer. The 1st reflection has been detected by the seismic method (the 1st red rectangular box in (c)). The 3rd reflection has been detected by the VSP method (the 3rd red rectangular box in (b)). The 4th reflection has not been detected by both methods.

Figure 73. The illustration of comparison of the seismic, the VSP, and the synthetic seismograms of an anticline-syncline model created using 5 sources for boundary 3. (a), (b), (c), and (d) show the retrieved stacked section, the VSP seismogram, the seismic seismogram, and the synthetic seismogram, respectively. The reflection from the coal layer has been detected by the seismic method (the 2 nd red rectangular box in (c)). The yellow arrow shows the climax of the interface of the coal layer. The 1st reflection has been detected by the seismic method (the 1st red rectangular box in (c)). The 3rd reflection has been detected by the seismic method (the 3 rd red rectangular box in (c)). The 4th reflection has not been detected by both methods....

Figure 74. The illustration of comparison of the seismic, the VSP, and the synthetic seismograms of an anticline-syncline model created using 5 sources for boundary 4. (a), (b), (c), and (d) show the retrieved stacked section, the VSP seismogram, the seismic seismogram, and the synthetic seismogram, respectively. The reflection from the coal layer has been detected by the seismic method (the 2 nd red rectangular box in (c)). The yellow arrow shows the climax of the interface of the coal layer. The 1st reflection has been detected by the seismic method (the 1st red rectangular box in (c)). The 3rd and the 4th reflections have not been detected by both methods

Figure 75. The illustration of comparison of the seismic, the VSP, and the synthetic seismograms of an anticline-syncline model created using 20 sources for boundary 1. (a), (b), (c), and (d) show the retrieved stacked section, the VSP seismogram, the seismic seismogram, and the synthetic seismogram, respectively. The reflection from the coal layer has been detected by the seismic method (the 2nd red rectangular box in (c)). The yellow arrow shows the climax of the interface of the coal layer. The 1st reflection has been detected by the seismic method (the red rectangular box in (c)). The 3rd reflection has been detected by the VSP method (the 3rd red rectangular box in (b)). The 4th reflection has not been detected by both methods... 113 
Figure 76. The illustration of comparison of the seismic, the VSP, and the synthetic seismograms of an anticline-syncline model created using 20 sources for boundary 2. (a), (b), (c), and (d) show the retrieved stacked section, the VSP seismogram, the seismic seismogram, and the synthetic seismogram, respectively. The reflection from the coal layer has been detected by the seismic method (the 2nd red rectangular box in (c)). The yellow arrow shows the climax of the interface of the coal layer. The 1st reflection has been detected by the seismic method (the red rectangular box in (c)). The 3rd reflection has been detected by the VSP method (the red rectangular box in (b)). The 4th reflection has not been detected by both methods................................................ 114

Figure 77. The illustration of comparison of the seismic, the VSP, and the synthetic seismograms of an anticline-syncline model created using 20 sources for boundary 3. (a), (b), (c), and (d) show the retrieved stacked section, the VSP seismogram, the seismic seismogram, and the synthetic seismogram, respectively. The reflection from the coal layer has been detected by the seismic method (the 2 nd red rectangular box in (c)). The yellow arrow shows the climax of the interface of the coal layer. The 1st reflection has been detected by the seismic method (the 1st red rectangular box in (c)). The 3rd reflection has been detected by the seismic method (the 3 rd red rectangular box in (c)). The 4 th reflection has not been detected by both methods........................................... 115

Figure 78. The illustration of comparison of the seismic, the VSP, and the synthetic seismograms of an anticline-syncline model created using 20 sources for boundary 4. (a), (b), (c), and (d) show the retrieved stacked section, the VSP seismogram, the seismic seismogram, and the synthetic seismogram, respectively. The reflection from the coal layer has been detected by the seismic method (the 2 nd red rectangular box in (c)). The yellow arrow shows the climax of the interface of the coal layer. The 1st reflection has been detected by the seismic method (the 1st red rectangular box in (c)). The 3rd reflection has been detected by the seismic method (the 3rd red rectangular box in (c)). The 4th reflection has not been detected by both methods......................................... 116

Figure 79. The illustration of comparison of the seismic, the VSP, and the synthetic seismograms of an anticline-syncline model created using 500 sources for boundary 1. (a), (b), (c), and (d) show the retrieved stacked section, the VSP seismogram, the seismic seismogram, and the synthetic seismogram, respectively. The reflection from the coal layer has been detected by the seismic method (the 2 nd red rectangular box in (c)). The yellow arrow shows the climax of the interface of the coal layer. The 1st reflection has been detected by the seismic method (the 1st red rectangular box in (c)). The 3rd reflection has been detected by both methods (the 3rd red rectangular box in (c) and in (b)). The 4th reflection has not been detected by both methods...................................117

Figure 80. The illustration of comparison of the seismic, the VSP, and the synthetic seismograms of an anticline-syncline model created using 500 sources for boundary 2. (a), (b), (c), and (d) show the retrieved stacked section, the VSP seismogram, the seismic 
seismogram, and the synthetic seismogram, respectively. The reflection from the coal layer has been detected by the seismic method (the 2 nd red rectangular box in (c)). The yellow arrow shows the climax of the interface of the coal layer. The 1st reflection has been detected by the seismic method (the 1st red rectangular box in (c)). The 3rd reflection has been detected by the VSP method (the 3rd red rectangular box in (b)). The 4th reflection has not been detected by both methods.

Figure 81. The illustration of comparison of the seismic, the VSP, and the synthetic seismograms of an anticline-syncline model created using 500 sources for boundary 3. (a), (b), (c), and (d) show the retrieved stacked section, the VSP seismogram, the seismic seismogram, and the synthetic seismogram, respectively. The reflection from the coal layer has been detected by the seismic method (the 2 nd red rectangular box in (c)). The yellow arrow shows the climax of the interface of the coal layer. The 1st reflection has been detected by the seismic method (the 1st red rectangular box in (c)). The 3rd reflection has been detected by the seismic method (the 3 rd red rectangular box in (c)). The 4th reflection has not been detected by both methods....

Figure 82. The illustration of comparison of the seismic, the VSP, and the synthetic seismograms of an anticline-syncline model created using 500 sources for boundary 4. (a), (b), (c), and (d) show the retrieved stacked section, the VSP seismogram, the seismic seismogram, and the synthetic seismogram, respectively. The reflection from the coal layer has been detected by the seismic method (the 2 nd red rectangular box in (c)). The yellow arrow shows the climax of the interface of the coal layer. The 1st reflection has been detected by the seismic method (the 1st red rectangular box in (c)). The 3rd reflection has been detected by the seismic method (the 3 rd red rectangular box in (c)). The 4 th reflection has been detected by the VSP method (the 4th red rectangular box in (b)) ..............120 


\section{List of tables}

Table 1. Acquisition parameters used in surface seismic ..............................................15

Table 2. Acquisition parameters used in zero-offset VSP ..........................................17

Table 3. Noise boundaries used for 5, 20, and 500 sources........................................19 


\section{Acknowledgements}

I would like to thank my advisor, Prof. Radwin Askari for his support and guidance through my research. His suggestions played an important role in shaping my work. Also, I would like to thank my committee members, Prof. Gregory P. White, and Dr. Nathan D. Manser for being on my thesis committee.

I also would like to thank Haitao Cao, who helped me and shared his scripts with me, and my friend Mohamed Shawky, who did not withhold his support in challenging times. I would like to thank my friend Erdem Cetin from Turkish Petroleum (TP), especially. He always helped me whenever I need some help about anything.

I am grateful to Dr. Jan Thorbecke at Delft University of Technology, Netherlands, who is the creator of the 2D Finite-Difference Wavefield Modeling computer code I used throughout my research. I consulted him many times and he responded to my questions with patience and sincerity.

I am thankful to Fahdi Maula from Schlumberger for his support regarding the generating Seismic Unix script for VSP processing. I would not be able to the VSP part without his assistance. I would like to thank also Utku Harmankaya and Ali Cankurtaranlar who always gave their support.

I would like to thank my beautiful country, The Republic of Turkey, for providing financial support to me, and the General Directorate of Mineral Research and Exploration (MTA) for their scholarship.

Finally, and most importantly, I would like to endless thank my family, who brought me up and who always supported me in challenging times. 


\section{Abstract}

Ambient noise seismic interferometry (ANSI) is a method that uses passive noise created by unknown sources such as local or teleseismic earthquakes, waves, wind, fracturing and fault movement in a reservoir to obtain an image of subsurface. ANSI has been used in some various disciplines, including earthquake engineering, time-lapse monitoring, borehole seismology and seismic exploration for years. Compared to conventional active seismic surveys, it is advantageous in terms of cost-efficiency as no active source such dynamite or vibroseis is used. Although many promising results have been obtained from surface wave ANSI, body wave ANSI is still challenging. Such a challenge might stem from the inherent lower amplitude of the body waves compared to the surface waves. To improve body ANSI, many methodologies based on seismic data processing and acquisition have been proposed.

The main objective of this study is to answer this question whether the zero-offset vertical seismic profiling (VSP) can provide additional information of subsurface in ANSI surveys if it is included along the conventional surface seismic data acquisition. Thus, I evaluate two different data acquisition techniques, surface seismic and zero-offset VSP data acquisitions for recovering body wave data from ANSI. To assess each of these data acquisition approaches on ANSI results, I created three different geological models (from more homogenous (simple) to more complex). In addition, for each geological model, I assumed different numbers of noise sources and noise boundaries using the finitedifference method. After retrieving reflection responses, the results obtained for surface seismic and zero-offset VSP were compared with the synthetic seismogram.

According to the results, when the geology is homogeneous (i.e., flat layering), the source distribution is wide and the number of point sources is high, the surface seismic data acquisition offers a better image of subsurface compare to VSP. However, once the geology becomes more complex, the source distribution is narrow, and the number of sources is limited, the VSP method can detect some of layers that have not been identified by the surface seismic method. Nevertheless, as I show, by combining the results from the two methods, we can obtain a better idea about subsurface layering. In other words, the two methods can complement each other. Thus, I propose the simultaneous surface seismic and zero-offset VSP data acquisitions for body wave ANSI. 


\section{Introduction}

Ambient noise seismic interferometry (ANSI) is a method based on constructing Green's function or seismic impulse response via the signals recorded by a pair of geophones (Schuster 2004; Wapenaar and Fokkema, 2006). Conventionally, the signal recorded by one receiver is cross correlated with the signal recorded by the other receiver. The resulted signal is a virtual seismic signal when the location of one of the receivers is regarded as the virtual source location and the location of the other receiver as the virtual receiver location.

The idea of seismic imaging using ANSI goes back to Claerbout (1968) who conjectures that signals from seismic sources randomly distributed in subsurface can provide an image of subsurface geological structures. Following Claerbout (1968), many studies have been conducted on ANSI. Weaver and Lobkis (2001) verified the possibility of seismic imaging via ANSI for ultrasound waves by assuming wavefield diffusivity. Wapenaar (2004) showed the possibility of ANSI imaging without the assumptions of wave field diffusivity. Derode et al. (2003) investigated the relationship between interferometry and time-reversed wavefields. Snieder (2004) demonstrated that direct source-receiver arrivals occur when the signal is stationary. Schuster et al. $(2001,2004)$ extended Claerbout's acoustic daylight imaging to arbitrary source distributions or any number of sources. Campillo and Paul (2003) conducted studies on the correlation of coda waves and impulse response reconstruction. Bakulin and Calvert (2004) performed a new approach that created a virtual source in borehole location. More developments of ANSI include surface tomography imaging (Shapiro and Campillo, 2004; Sabra et al., 2005), regional tomography imaging (Shapiro et al., 2005; Yao et al., 2006), and monitoring the inner parts of volcanoes (SensSchönfelder and Wegler, 2006; Brenguier et al., 2008).

Researchers also used seismic interferometry to retrieve body waves through passive seismic data (e.g., Ravi Kumar and Bostock, 2006; Tonegawa et al., 2009; Ruigrok et al., 2011; Roux et al., 2005; Hohl and Mateeva, 2006; Draganov et al., 2007; Draganov et al., 2009; Zhan et al., 2010; Ruigrok et al., 2011; Xu et al., 2012). Recently, Sager et al. (2018) investigated the full waveform ambient noise inversion that aims to extract the Green's function. Sager et al. (2020) presented the first application about full-waveform ambient noise inversion to image subsurface. Tkalčić et al. (2020) utilizes coda wavefield to investigate the earth's deep structure in earthquake seismology. Fichtner at al. (2016) developed an extensive full-waveform theory that can be used for seismological data.

Recently, some remarkable studies on ANSI have been carried out, particularly regarding $\mathrm{CO}_{2}$ sequestration. One of these studies was carried out at the Ketzin $\mathrm{CO}_{2}$ storage site, Germany, and the ANSI method succeeded in obtaining a reflection image from body wave noise (Paap et al., 2014, Xu et al., 2012). Boullenger et al. (2015) verified the ability of the ANSI method to monitor the impedance changes initiated from $\mathrm{CO}_{2}$ injection. Tsuji et al. (2016) applied three correlation techniques to passive data during water injection operations reservoir monitoring. Zhou et al. (2017) retrieved both P-waves and S-waves for reservoir monitoring from ANSI data. Cao and Askari (2019) compared three methods 
of cross-correlation, deconvolution, and cross-coherence in ambient noise seismic interferometry (ANSI) to monitor the impedance variations during $\mathrm{CO}_{2}$ sequestration.

Seismic interferometry is also used in borehole seismology, which might provide a higher resolution of subsurface compared to the surface seismic acquisition method. He et al. (2006) and Jiang et al. (2007) investigated interferometric migration using VSP multiples. Several works are conducted to image flanks using VSP data (Lu et al., 2008; Hornby and Yu et al., 2007; Xiao et al., 2006; Willis et al., 2006). Wang et al. (2010) noted that the primary waves and ghosts together can display subsurface reflectors using the walk away VSP data. Matsushima et al. (2016) examined the attenuation prediction with the help of zero-offset VSP data. Bharadwaj et al. (2018) developed a new method called focused blind deconvolution (FBD), demonstrating the advantages of this method, which uses synthetic seismic during drilling works nearby and ahead of borehole.

Numerical modeling is an important tool to optimize various acquisition scenarios to improve seismic imaging via ANSI. Thorbecke and Draganov (2011) investigated the effects of several parameters such as a number of sources, noise recording time, and source locations through a 2D finite-difference wavefield modeling code (fdelmode) (Thorbecke, 2011). Given the promising results of VSP ANSI, the purpose of this study is to determine when and how the zero-offset VSP data acquisition can contribute to subsurface imaging and complement the results obtained from conventional surface seismic data acquisition. To this aim, I assume four different noise boundaries, and the three geological models. In addition, I consider various number of sources for a given geological model and source distribution. I use fdelmode to generate ambient noise data. To retrieve body wave data, I apply the conventional cross-correlation technique. After doing the necessary data processing steps for surface seismic and zero offset VSP methods, the reflection responses of the subsurface layers are obtained. The outputs of two methods are compared to evaluate their performances to image subsurface structures. 


\section{Theory}

\subsection{Green's Function in Seismic Interferometry}

Seismic interferometry is a method predicting the Green's function between two receivers by cross-correlation of the traces recorded in these receivers and stacking of these traces (Wapenaar and Fokkema, 2006). For an inhomogeneous and lossless acoustic medium if we denote Green's function by $G\left(x, x_{A}, t\right)$, where $x$ and $x_{A}$ are the source position and geophone locations at the surface respectively, and $t$ denotes time, then the Fourier Transform is of Green's function is

$$
\widehat{G}\left(x, x_{A}, t\right)=\int_{-\infty}^{\infty} \exp (-j w t) G\left(x, x_{A}, t\right) d t
$$

If we assume that there is a point source in $x_{A}$, the acoustic pressure $G\left(x, x_{A}, t\right)$ is given by

$$
\rho \partial_{i}\left((1 / \rho(x)) \partial_{i} \hat{G}\left(x, x_{A}, w\right)\right)+\left(w^{2} / c^{2}(x)\right) \hat{G}\left(x, x_{A}, w\right)=-j w \rho \delta\left(x-x_{A}\right)
$$

where $c(x)$ and $\rho(x)$ are wave propagation velocity and mass density, respectively. $\partial_{i}$ denotes partial derivative in the $\mathrm{x}_{\mathrm{i}}$ direction. Using the Rayleigh reciprocity theorem (Rayleigh, 1878; Bojarski, 1983; Wapenaar et al., 2004, 2005), $\hat{G}$ 's representation is given by

$$
\begin{gathered}
\widehat{G}_{h}\left(x_{A}, x_{B}\right)=\oint_{\partial D} \frac{-1}{j \omega \rho(x)}\left(\hat{G}^{*}\left(x_{A}, x\right) \partial_{i} \hat{G}\left(x_{B}, x\right)-\left(\partial_{i} \hat{G}^{*}\left(x_{A}, x\right)\right) \hat{G}\left(x_{B}, x\right)\right) n_{i} d^{2} x \\
\hat{G}_{h}\left(x_{A}, x_{B}\right) \triangleq \hat{G}\left(x_{A}, x_{B}\right)+\hat{G}^{*}\left(x_{A}, x_{B}\right)=2 \mathfrak{R}\left\{\widehat{G}\left(x_{A}, x_{B}\right)\right\}
\end{gathered}
$$

where $\partial D$ is a closed surface, $n$ is a normal vector, $*$ denotes complex conjugation, and $x_{A}$ and $x_{B}$ are presented inside $\partial D$. However, Equation 4 is applied for lossless inhomogeneous acoustic medium. To make Equation 4 more suitable, some approaches have been introduced. For example, it is assumed that the medium is homogeneous and the parameters around this surface change smoothly, outside at the closed surface and along the closed surface (Wapenaar and Fokkema, 2006) that can be parameterized as

$$
2 \Re\left\{\widehat{G}\left(x_{A}, x_{B}\right)\right\} \approx \frac{2}{c \rho} \oint_{\partial D} \hat{G}^{*}\left(x_{A}, x\right) \hat{G}\left(x_{B}, x\right) d^{2} x
$$

Such an assumption might cause amplitude errors (Ramirez and Weiglein, 2009) and spurious issues (Wapenaar and Fokkema, 2006). However, since the phase is not affected, this approach can be used for seismic interferometry. If the sources are uncorrelated in time and space, the observed wavefields can be written as in equation 6 as follows: 


$$
\begin{aligned}
& \hat{u}^{o b s}\left(x_{A}\right)=\oint_{\partial D} \widehat{G}\left(x_{A}, x\right) \widehat{N}(x) d^{2} x \\
& \hat{u}^{o b s}\left(x_{B}\right)=\oint_{\partial D} \widehat{G}\left(x_{B}, x\right) \widehat{N}(x) d^{2} x
\end{aligned}
$$

where $\widehat{N}(x, \omega)$ is the noise spectrum that fulfills Equation 7

$$
\left\langle\widehat{N}(x) \widehat{N}^{*}\left(x^{\prime}\right)\right\rangle=\delta\left(x-x^{\prime}\right) \hat{S}(\omega)
$$

where $\langle$.$\rangle corresponds to the spatial ensemble average, \hat{S}(\omega)$ is the power spectrum of the sources. Since $\widehat{N}(x)$ and $\widehat{N}^{*}\left(x^{\prime}\right)$ are mutually uncorrelated noises, and $\hat{S}(\omega)$ is the same for all $x$ cartesian coordinates. Using Equation 6 and 7 in equation 5 results in

$$
2 \Re\left\{\hat{G}\left(x_{A}, x_{B}\right)\right\} \hat{S}(\omega) \approx \frac{2}{c \rho}\left\langle\hat{u}^{o b s *}\left(x_{A}\right) \hat{u}^{o b s}\left(x_{B}\right)\right\rangle
$$

Equation 8 is the fundamental equation of the cross correlation of the observed wavefields for generating the Green's function or retrieve reflection response from passive source measurements for a receiver at point A with a source at point B (Wapenaar and Fokkema, 2006).

\subsection{Cross-correlation Method}

There are some different correlation methods used in seismic interferometry like crosscorrelation, cross-coherence, deconvolution, focused blind deconvolution, coda correlation, and waveform cross-correlation. Some differences between cross-correlation, cross-coherence, and deconvolution methods regarding resolution are shown by Cao and Askari (2019). In our study, the cross-correlation method was used. With the crosscorrelation method, signals from underground sources are recorded by two stations (geophones), and Green's function is extracted (Weaver and Lebkis, 2002; Derode et al., 2003; Wapenaar, 2004; Larose et al., 2006; Curtis et al., 2006; Nakahara, 2006).

Figure 1 shows the concept described above simply. A wavefield emitted from a subsurface source is recorded by the geophone when the wavefield reaches the earth's surface (Figure 1a). Then, when the wave reflected from the earth's surface back to the subsurface, it is again reflected to the earth's surface by a subsurface reflector and recorded by the other geophone (Figure 1b). The traces recorded by two geophones are cross-correlated, the common path is canceled and the path from the first receiver to the reflector and from the reflector to the second receiver remains. Thus, the seismic reflection response or Green's 
function observed by the second geophone is obtained as if there was a source in the location of the first geophone (Figure 1c). Therefore, a virtual shot gather is resulted in a single geophone location (Wapenaar et al., 2010a, and 2010b).
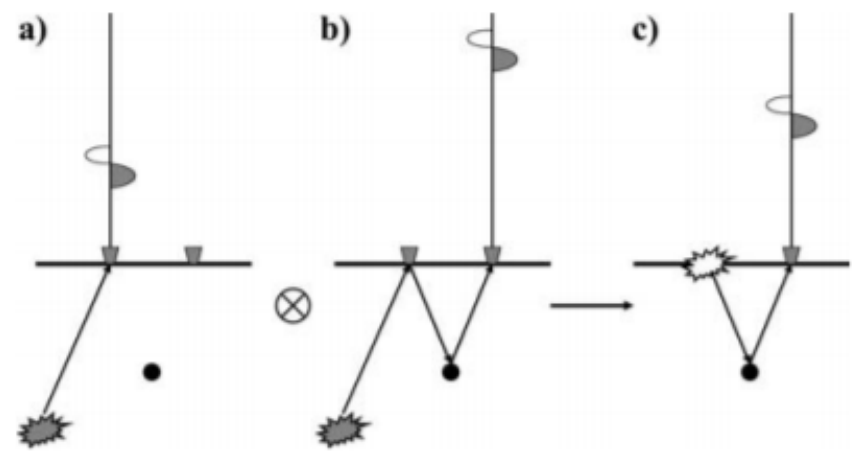

Figure 1. The fundamental principle of reflected-wave interferometry (Schuster, 2001, 2009). (a) The subsurface source is emitted a signal from underground to the surface and is recorded by a geophone. (b) A second geophone receives the signal by an underground scatterer. (c) The cross-correlation cancels the common path from the source to the first receiver.

\subsection{D Finite-Difference Wavefield Modeling Software}

I used an open source 2D finite-difference wavefield modeling (fdelmodc) program developed to produce seismic waves from source points that are located below the surface. This code is based on the use of Seismic Unix interface. The fdelmodc can be used for acoustic, elastic, visco-acoustic, and visco-elastic modeling. In this study, I used acoustic modeling. When the fdelmodc performing modeling, it calculates the 2D wave equation (Equations 9-10-11) approximating the derivatives in the wave equation with the help of finite differences. In the 2D wave equation, first order linearized components are defined by the Newton and Hooke law. The equations used for the acoustic medium are

$$
\begin{gathered}
\frac{\partial V x}{\partial t}=-\frac{1}{\rho} \frac{\partial P}{\partial x^{\prime}} \\
\frac{\partial V z}{\partial t}=-\frac{1}{\rho} \frac{\partial P}{\partial z^{\prime}} \\
\frac{\partial P}{\partial t}=-\frac{1}{\kappa}\left\{\frac{\partial V x}{\partial x}+\frac{\partial V z}{\partial z}\right\},
\end{gathered}
$$


where $V_{x}$ and $V_{z}$ the components of the particle velocities in the $\mathrm{x}$ and $\mathrm{z}$ directions, $P$ is the acoustic pressure, $\rho$ is the density of the medium, and $\kappa$ is the compressibility.

To calculate the partial derivative in a grid point, four grid numbers are required (Thorbecke, 2019) and is given by

$$
\frac{27 D_{1}-D_{2}}{24 \Delta x} \approx \frac{\partial P}{\partial x}+0 \Delta x^{4} \approx \frac{27\left(P\left(x+\frac{\Delta x}{2}\right)-P\left(x-\frac{\Delta x}{2}\right)\right)-P\left(x+\frac{3 \Delta x}{2}\right)+P\left(x-\frac{3 \Delta x}{2}\right)}{24 \Delta x}+0 \Delta x^{4}
$$

The medium parameters used in the fdelmodc program are given by

$$
\begin{aligned}
& (\lambda+2 \mu)=c^{2}{ }_{P} \rho=\frac{1}{\kappa} \\
& \mu=c^{2}{ }_{S} \rho
\end{aligned}
$$

where $\rho$ is the medium density, $c_{P}$ is the P-wave velocity, $c_{S}$ is the $\mathrm{S}$-wave velocity, $\lambda$ and $\mu$ are the Lame parameters, and $\kappa$ is the compressibility. Fdelmodc can simulate an ANSI data acquisition for given recording time, source distribution and number of sources for (Thorbecke and Draganov, 2011). 


\section{Methodology}

In this section, first, I explain the parametrization used in 2D finite-difference wavefield modeling code (Thorbecke, 2011). Then, I present three geological models, which are a flat-layered model, anticline model with a moderate steep and an anticline with a high steep. In addition, I show different sources distributions that I used for my modeling from deep to the surface. While doing this, four different noise boundaries are created, and models are retrieved with these randomly distributed noise sources in these boundaries. These four boundaries are used for two different geometries, which are surface and zerooffset VSP. Each trace in the noise panel obtained after modeling is cross correlated with all other traces. Finally, different data processing steps that I applied to ANSI data for surface and VSP data acquisitions are presented.

\subsection{D Finite Modeling}

In the 2D finite-difference (FD) method, we should be aware of the possibility of generating some artifacts such as dispersion. If the conditions given in Equation 15 are not met, FD will produce dispersed waves. This is controlled by the $f_{\text {max }}$ parameter used in the program. In such a case, by choosing a frequency higher than the maximum frequency in the source wavelet, the dispersion issue can be resolved (Thorbecke, 2019).

$$
\begin{gathered}
\Delta h<\frac{c_{\min }}{5 f_{\max }} \\
\Delta h<\frac{\lambda_{\min }}{5}
\end{gathered}
$$

where $c_{\min }$ denotes the minimum $\mathrm{P}$-wave velocity, $f_{\max }$ represents the maximum frequency of the signal coming from sources, $\lambda_{\min }$ corresponds to minimum wavelength, and $\Delta h$ corresponds to the discretization step.

For a given maximum frequency, the FD code can generate seismic wavelet with different durations. Figure $2 \mathrm{a}$ shows 20 random source signatures with varying time duration (the maximum time is $5 \mathrm{~s}$ ) and Figure $2 \mathrm{~b}$ shows one source's signature, Figure $2 \mathrm{c}$ shows the amplitude spectrum of the random source signature. We note that in this case, the maximum frequency is set $24 \mathrm{~Hz}$ to avoid dispersion. In addition, the beginning and end of the signals are smoothly extrapolated to further avoid dispersion and suppress high frequencies (see Figures $3 \mathrm{a}$ and $3 \mathrm{~b}$ ). Noise signals are generated by assigning random values to the amplitude and phase values of the source signal according to the given maximum frequency $\left(f_{\max }\right)$ (Thorbecke and Draganov, 2011). The source signature of the noises is starting from zero amplitude and ending at zero amplitude (Figure $2 \mathrm{~b}$ ). 
(a)

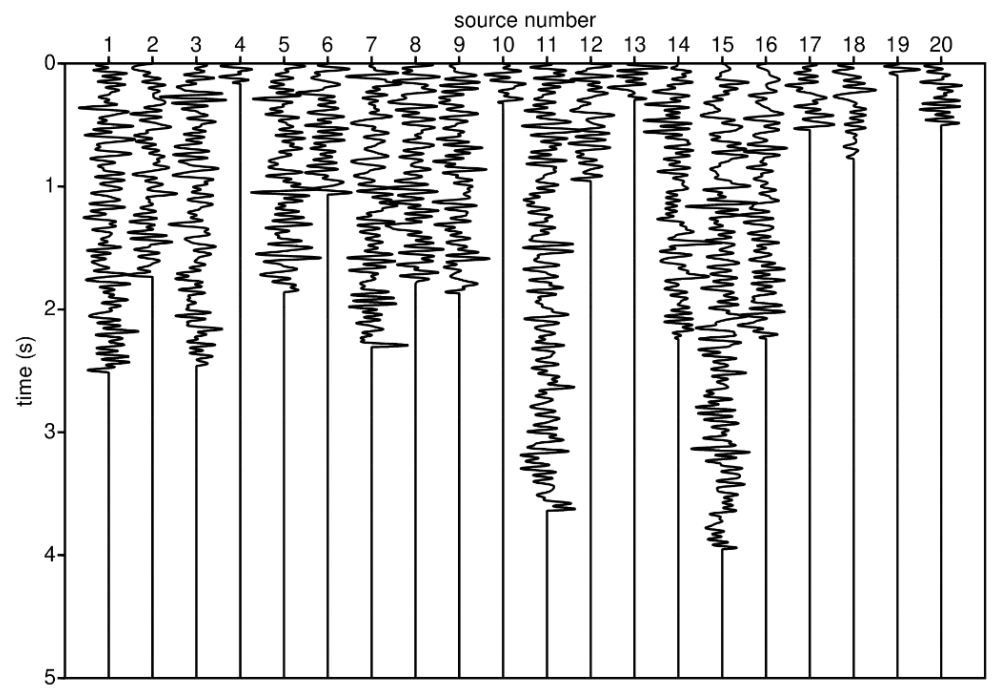

(b)

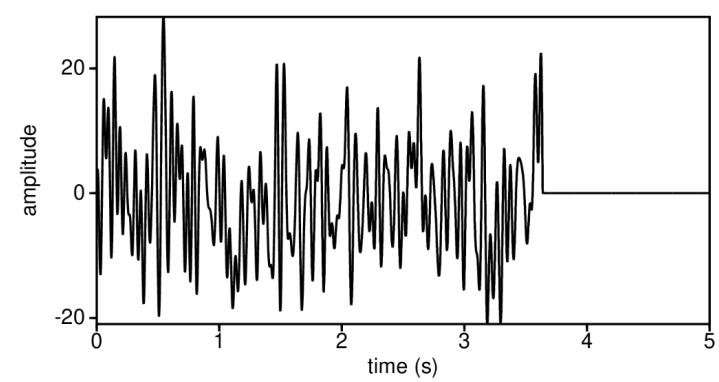

(c)

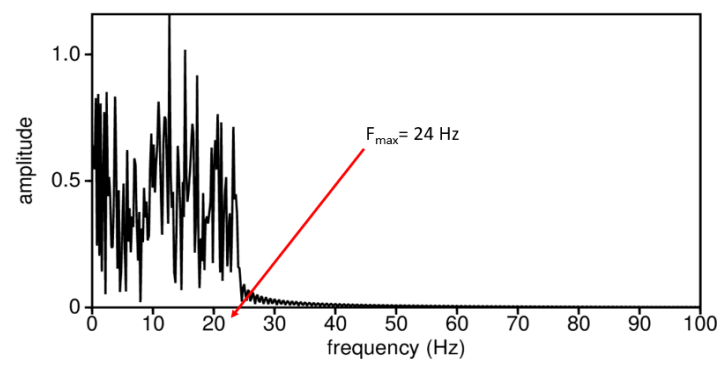

Figure 2. (a) The 20 random sources signatures with varying source duration. (b) One source signature. (c) The amplitude spectrum of the source signature in (b). 
(a)

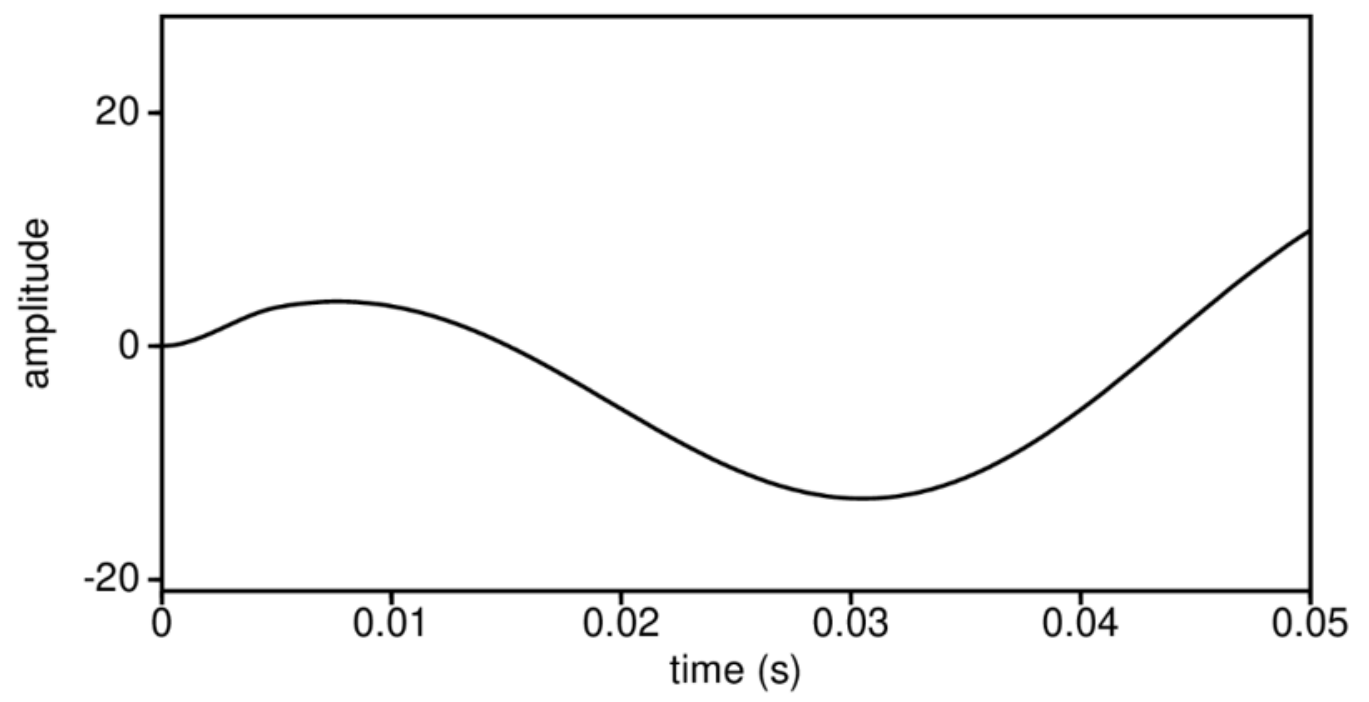

(b)

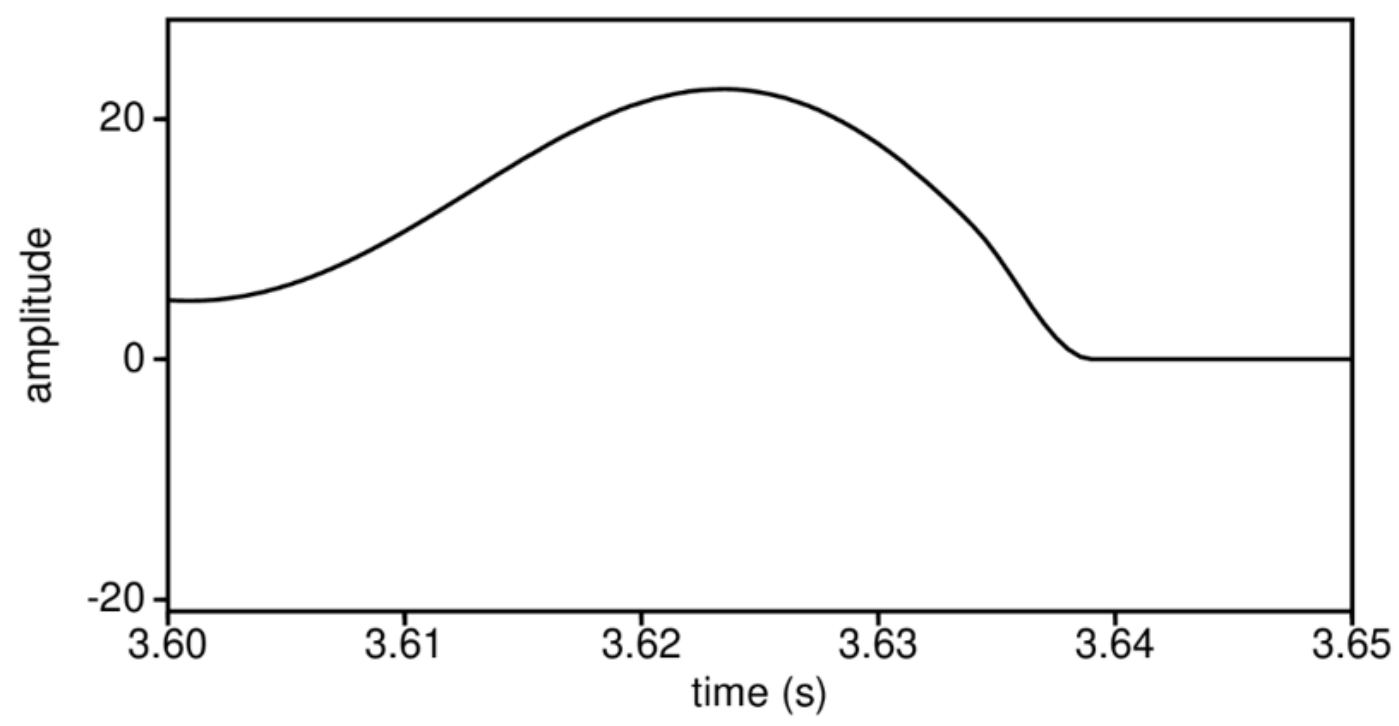

Figure 3. (a) shows the beginning and (b) shows the end of the signal amplitude presented in Figure 2b. 


\subsubsection{Geological models}

I consider three different geological models (Figure 4, Figure 5, and Figure 6), which are flat, anticline and anticline-syncline models. These models have different level of complexity from a homogenous (i.e., flat layering) to more complex (i.e., anticlinesyncline). Layer 3 is assigned as a coal/reservoir layer in these models with proper velocity and density values (the area indicated by a yellow box in Figures 4, 5, and 6). 


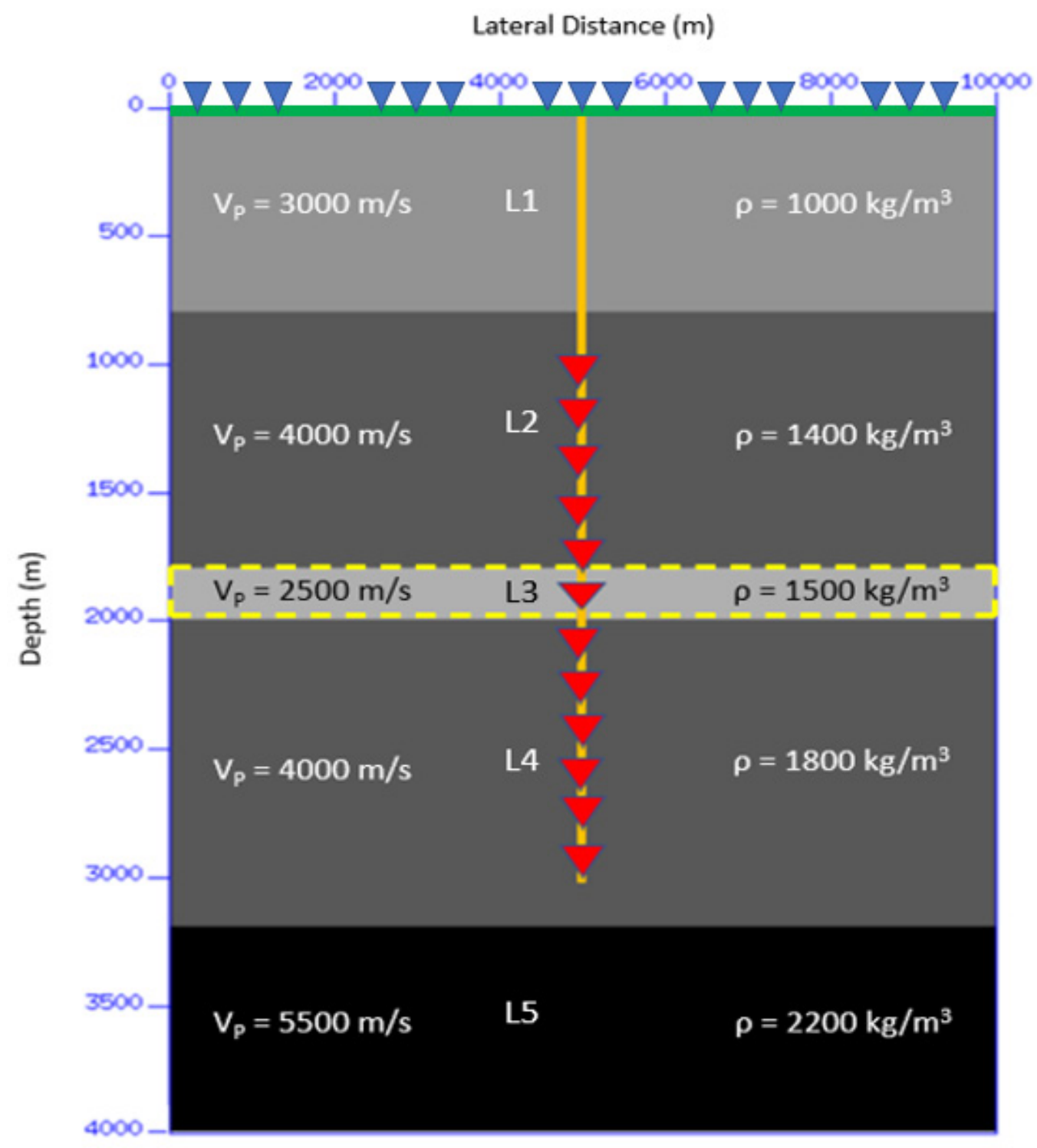

Figure 4. An acoustic velocity model with flat layers that are combined surface seismic and zero-offset VSP geometry. The green lateral line shows geophone array for surface seismic geometry. Blue triangles $(\nabla)$ correspond to 201 geophones that are placed on the free surface. L3 (covered by yellow-dashed area) corresponds to the coal layer. The geophones are layered on the free surface between $0 \mathrm{~m}$ to $10,000 \mathrm{~m}$. The vertical orange line shows VSP line, the red triangles $(\nabla)$ (101geophones) show the array of the geophones, which are layered between 1,000 $\mathrm{m}$ and 3,000 $\mathrm{m}$, used in the VSP geometry. 


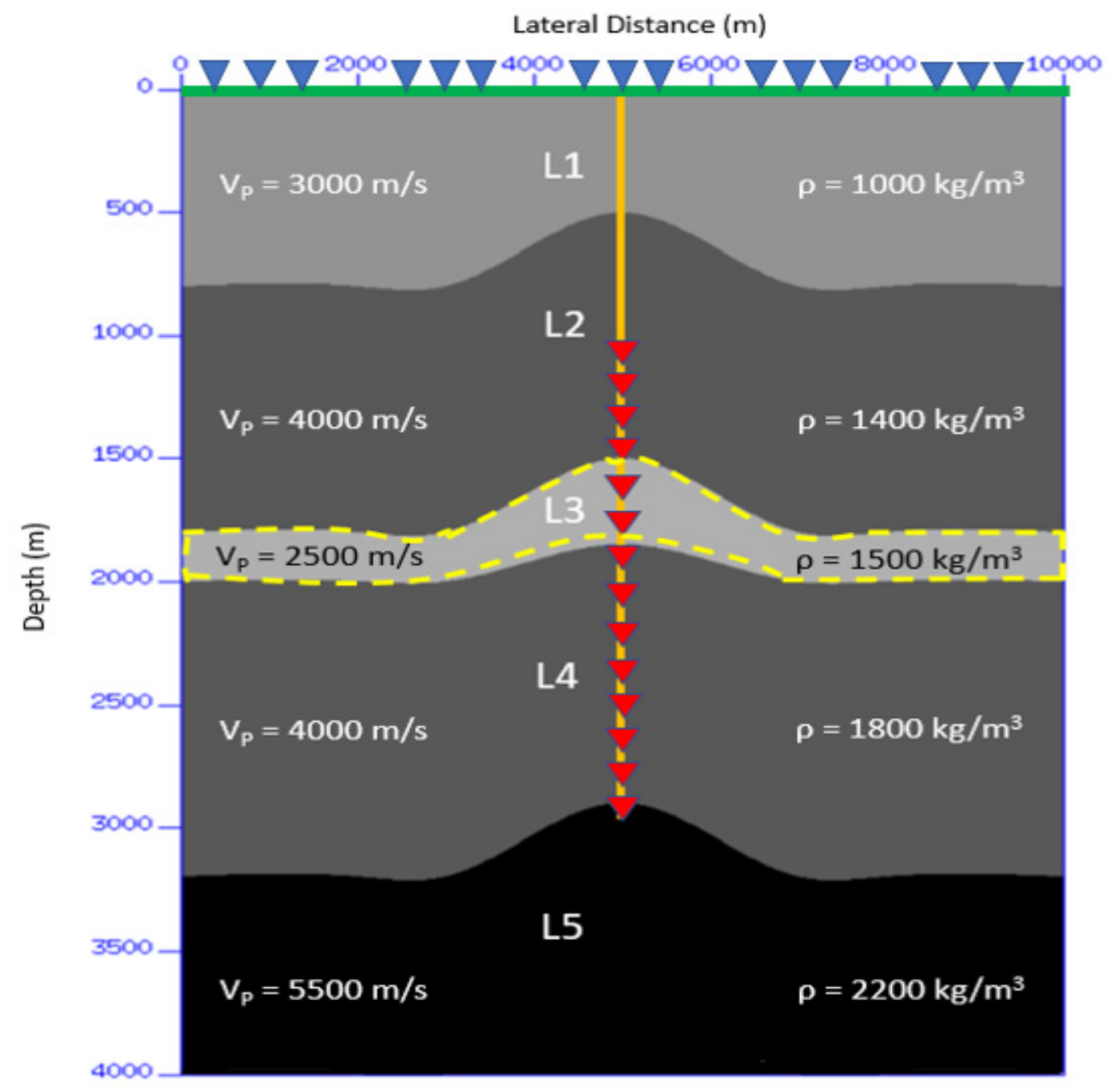

Figure 5. An acoustic velocity model with anticline layers that are combined surface seismic and zero-offset VSP geometry. The green lateral line shows geophone array for surface seismic geometry. Blue triangles $(\nabla)$ correspond to 201 geophones that are placed on the free surface. L3 (covered by yellow-dashed area) corresponds to the coal layer. The geophones are layered on the free surface between $0 \mathrm{~m}$ to $10,000 \mathrm{~m}$. The vertical orange line shows VSP line, the red triangles $(\nabla)$ (101 geophones) show the array of the geophones, which are layered between 1,000 $\mathrm{m}$ and 3,000 $\mathrm{m}$, used in the VSP geometry. 


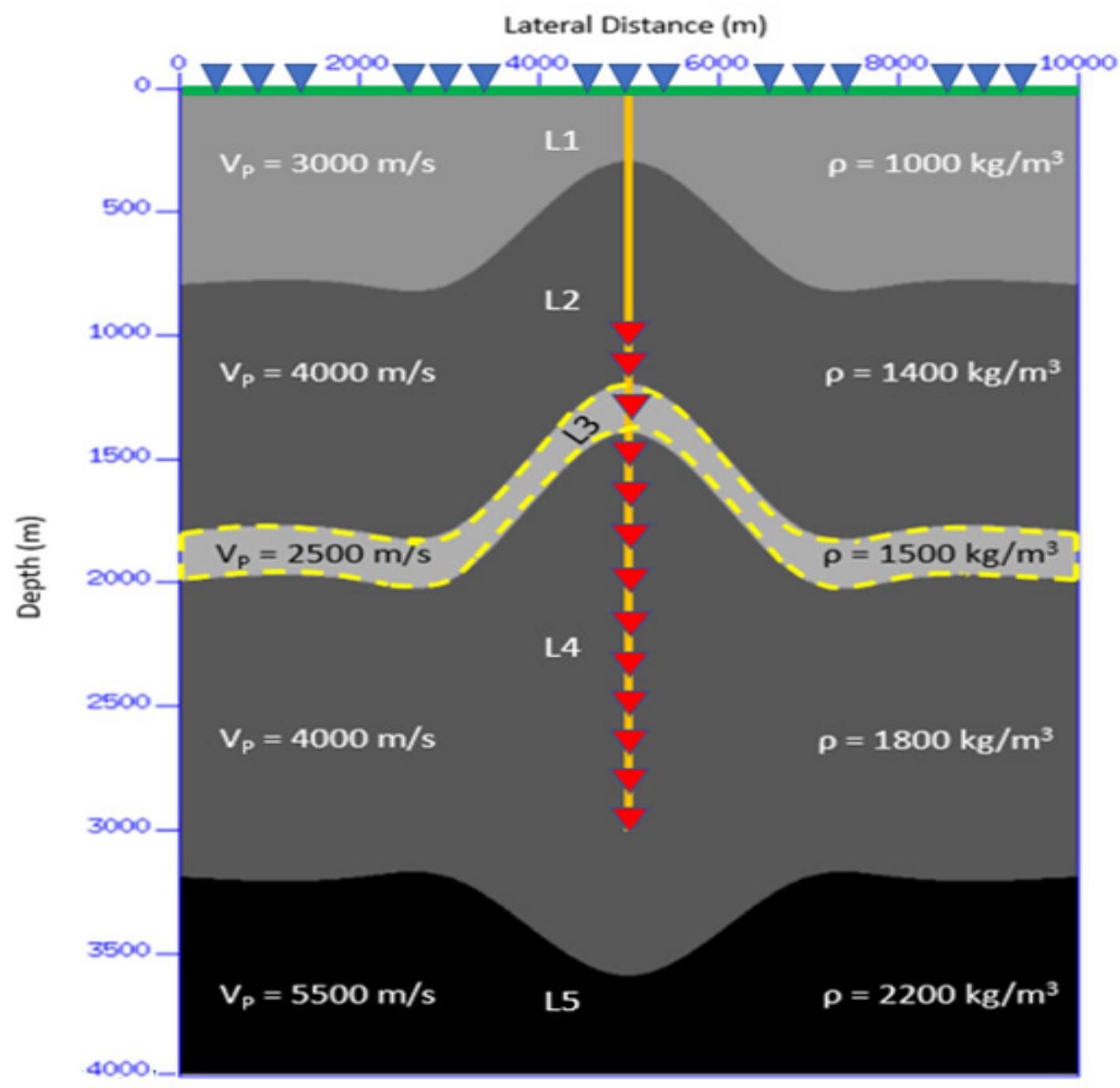

Figure 6. An acoustic velocity model with 3 anticlines and 1 syncline that are combined surface seismic and zero-offset VSP geometry. The green lateral line shows geophone array for surface seismic geometry. Blue triangles $(\nabla)$ correspond to 201 geophones that are placed on the free surface. L3 (covered by yellow-dashed area) corresponds to the coal layer. The geophones are layered on the free surface between $0 \mathrm{~m}$ to $10,000 \mathrm{~m}$. The vertical orange line shows VSP line, the red triangles $(\nabla)$ (101geophones) show the array of the geophones, which are layered between 1,000 $\mathrm{m}$ and 3,000 $\mathrm{m}$, used in the VSP geometry. 


\subsubsection{Surface data acquisition and processing}

In surface seismic data processing, for a given geological model, I created noise panels. Then, by applying cross-correlation, I obtained virtual shot gathers. In the next step, the virtual shot gathers were merged and sorted into CMP gathers. After performing velocity analysis, NMO correction was applied and events in CMP gathers were flattened. In the last step, all traces were stacked and retrieved reflection response of the underground (for anticline model, flat model, and anticline-syncline model). All these steps performed are shown in Figure 7. The acquisition parameters used for the surface seismic are also shown in Table 1.

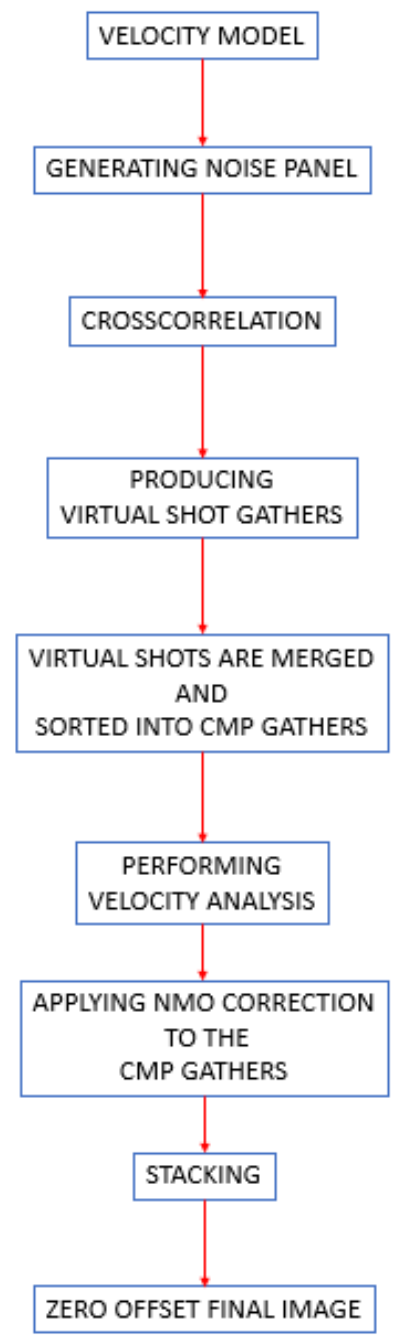

Figure 7. The processing flow of the surface seismic interferometry 
Table 1. Acquisition parameters used in surface seismic

\begin{tabular}{ll} 
PARAMETERS & VALUES \\
\hline Number of Sources & $5-20-500$ \\
Source Spacing & $10 \mathrm{~m}$ \\
The First Source's Depth & $100 \mathrm{~m}$ \\
The Last Source's Depth & $3,900 \mathrm{~m}$ \\
Number of Receivers & 201 \\
Receiver Interval & $50 \mathrm{~m}$ \\
Sampling Rate & $0.008 \mathrm{sec}$ \\
High-cut Frequency & $24 \mathrm{~Hz}$ \\
Recording Time & $120 \mathrm{sec}$ \\
\hline
\end{tabular}

\subsubsection{Zero-Offset VSP}

For the zero-offset VSP data acquisition, the geophones are assumed to be in a borehole within the centers of the geological models. The VSP line has an extension on from 1,000 $\mathrm{m}$ to $3,000 \mathrm{~m}$ below the surface. The number of receivers and receiver interval change with respect to the acquisition parameters used in the zero-offset VSP (Table 2). The vertical distance between geophones is $20 \mathrm{~m}$, resulting in 101 geophones. As a result of cross correlation, 101 traces and 101 virtual shot gathers will be generated.

I applied several data processing steps to the data from zero-offset VSP interferometry. First, I picked energy centers on the zero-amplitude line corresponding to peak time. Then, I separated wavefields by frequency-wavenumber filtering method. After separation, static correction was done. In the next step, upgoing wavefield was flattened. Thereby, the upgoing waves are shifted by the picked times to obtain two-way travel time. Finally, corridor stacking was performed. After these processing steps, the reflection response of the underground is obtained. The workflow for the zero-offset VSP has been outlined in in Figure 8 . 


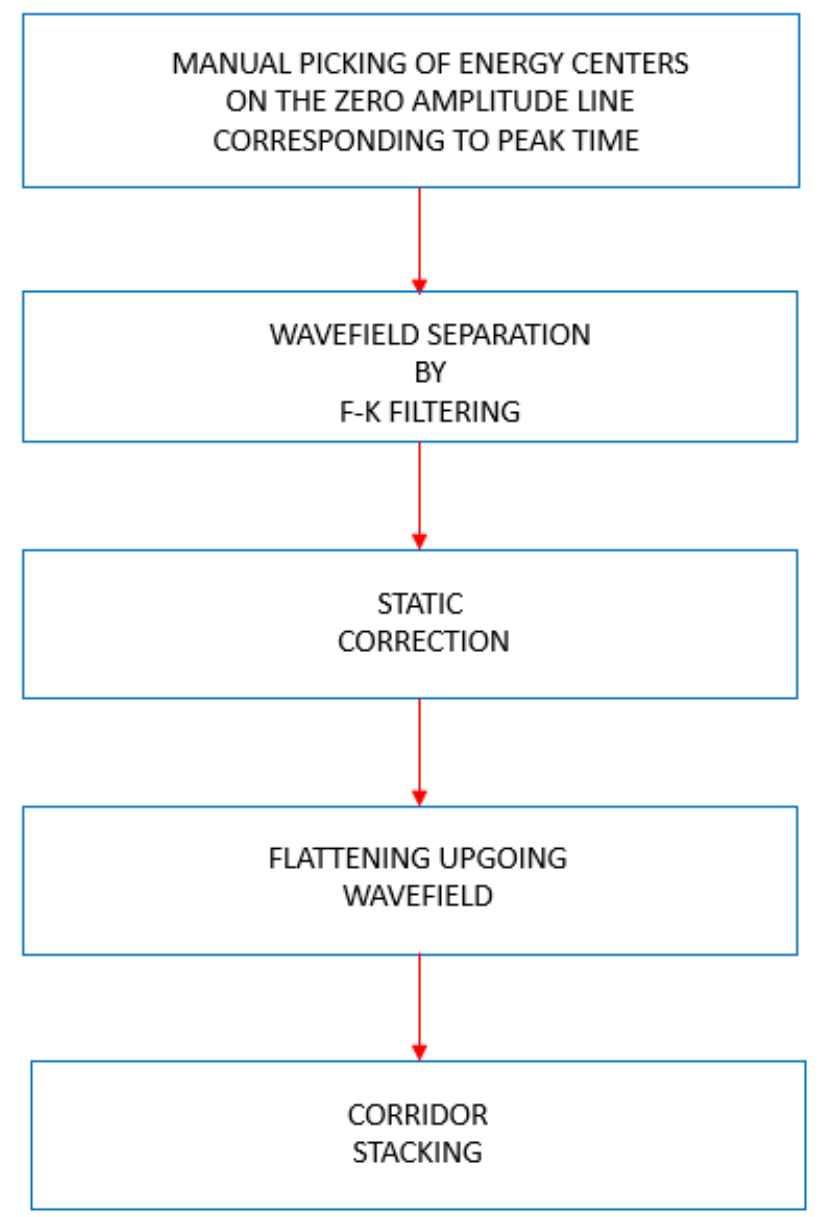

Figure 8 . The processing flow of the zero-offset VSP interferometry 
Table 2. Acquisition parameters used in zero-offset VSP

\begin{tabular}{ll} 
PARAMETERS & VALUES \\
\hline Number of Sources & $5-20-500$ \\
Source Spacing & $10 \mathrm{~m}$ \\
The First Source's Depth & $100 \mathrm{~m}$ \\
The Last Source's Depth & $3,900 \mathrm{~m}$ \\
Number of Receivers & 101 \\
Receiver Interval & $20 \mathrm{~m}$ \\
Sampling Rate & $0.008 \mathrm{sec}$ \\
High-cut Frequency & $24 \mathrm{~Hz}$ \\
Recording Time & $120 \mathrm{sec}$ \\
\hline
\end{tabular}

\subsection{Noise Boundaries}

In study, I consider four noise boundaries in which I assume the noise sources are located. While the depth ranges of these boundaries are the same $(100 \mathrm{~m}$ to $3,900 \mathrm{~m})$, their horizontal ranges are different (Figure 9 and Table 3). Noise boundary 1 ranges from 4,500 $\mathrm{m}$ to $5,500 \mathrm{~m}$ (horizontal direction); noise boundary 2 from 4,000 $\mathrm{m}$ to $6,000 \mathrm{~m}$; noise boundary 3 from $2,000 \mathrm{~m}$ to $8,000 \mathrm{~m}$, and noise boundary 4 from $100 \mathrm{~m}$ to $9,900 \mathrm{~m}$. For a given geological model and noise boundary, I consider three numbers of source $(5,20$, and 500). Sources are simultaneously excited and recorded by geophones for a duration of 120 seconds.

72 seismic sections are obtained from four noise boundaries, three number of noise sources, and three geological models based on surface and zero-offset VSP data acquisitions. 


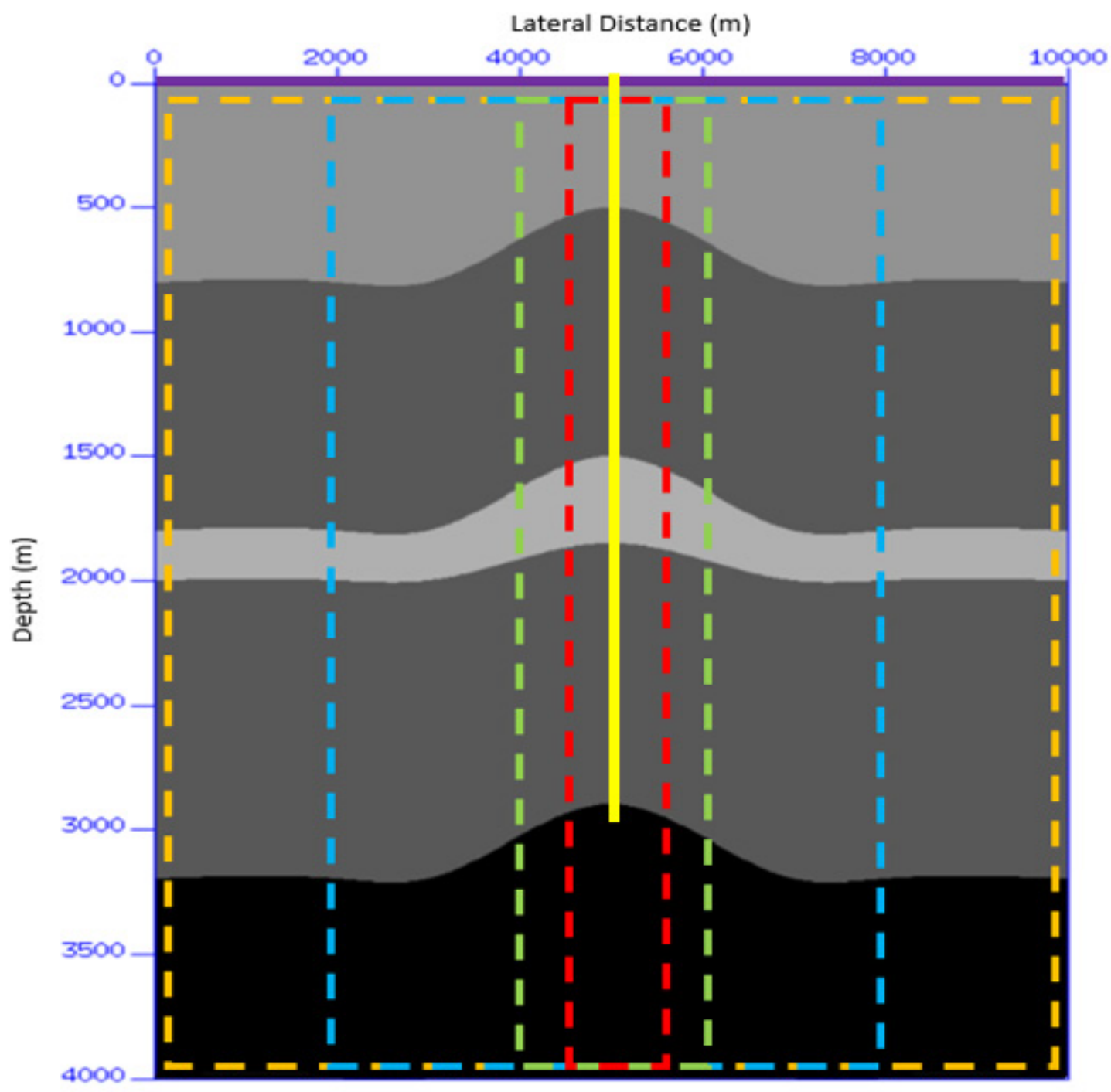

Figure 9. Four different noise boundaries for surface seismic geometry and zero-offset VSP geometry used in this study. The lateral horizontal dark purple line on the surface shows the surface seismic geometry. The vertical thick yellow line shows the VSP tool positioned $(5,000 \mathrm{~m}, 3,000 \mathrm{~m})$. The noise boundary 1 (vertical red dashed lines) laterally extends from $4,500 \mathrm{~m}$ to $5,500 \mathrm{~m}$; the noise boundary 2 (vertical green dashed lines) laterally extends from $4,000 \mathrm{~m}$ to $6,000 \mathrm{~m}$; the noise boundary 3 (vertical blue dashed lines) laterally extends from $2,000 \mathrm{~m}$ to $8,000 \mathrm{~m}$, and the noise boundary 4 (vertical orange dashed lines) laterally extends from $100 \mathrm{~m}$ to $9,900 \mathrm{~m}$. 
Table 3: Noise boundaries used for 5, 20, and 500 sources

Noise Boundary

1

2

3

4
Lateral Extent $(\mathrm{m})$

$4,500-5,500$

$4,000-6,000$

$2,000-8,000$

$100-9,900$
Vertical Extent (m)

$100-3,900$

$100-3,900$

$100-3,900$

$100-3,900$

\subsection{The Cross-correlation of Zero-Offset VSP and Seismic Interferometry}

After running the model, a cross-correlation procedure is carried out. At this stage, noise panels are produced for surface seismic geometry. In surface seismic geometry, before cross-correlation, RMS normalization is performed to smooth by the amplitude variations in noisy data. Thus, an equal contribution is obtained from each trace. Then, cross correlation is applied to the normalization data. To obtain the correlation panel, a trace from the data is extracted as a master trace, which is the trace in the position in which a virtual source is located. The virtual shot gathers are obtained by cross correlating this master trace with all other traces. Figure 10 shows the cross-correlation procedure in surface seismic geometry.
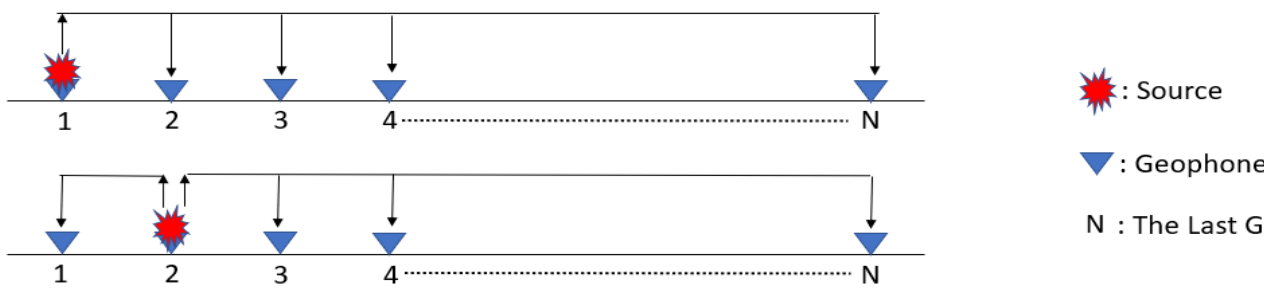

$\mathrm{N}$ : The Last Geophone

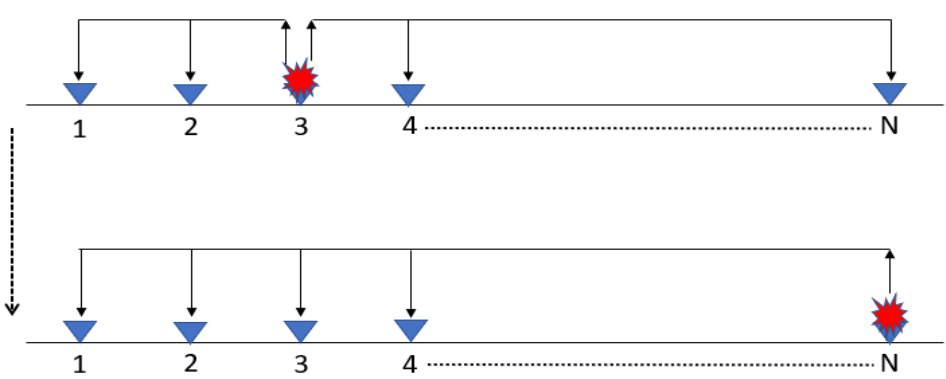

Figure 10. The surface seismic cross-correlation procedure in seismic interferometry. $(\nabla)$ represents the geophone, while $\left({ }_{m}\right)$ corresponds to the source. $\mathrm{N}$ denotes the last geophone. 
To show how a virtual shot record from surface data is generated, I obtained a noise panel by using 20 sources within boundary 1 in the anticline seismic model (Figure 11a). From noise panel, a master trace, which is trace 101, is selected (Figure 11b). Then, the master trace is cross correlated with other traces to obtain a virtual shot record (Figure 11c).

(a)

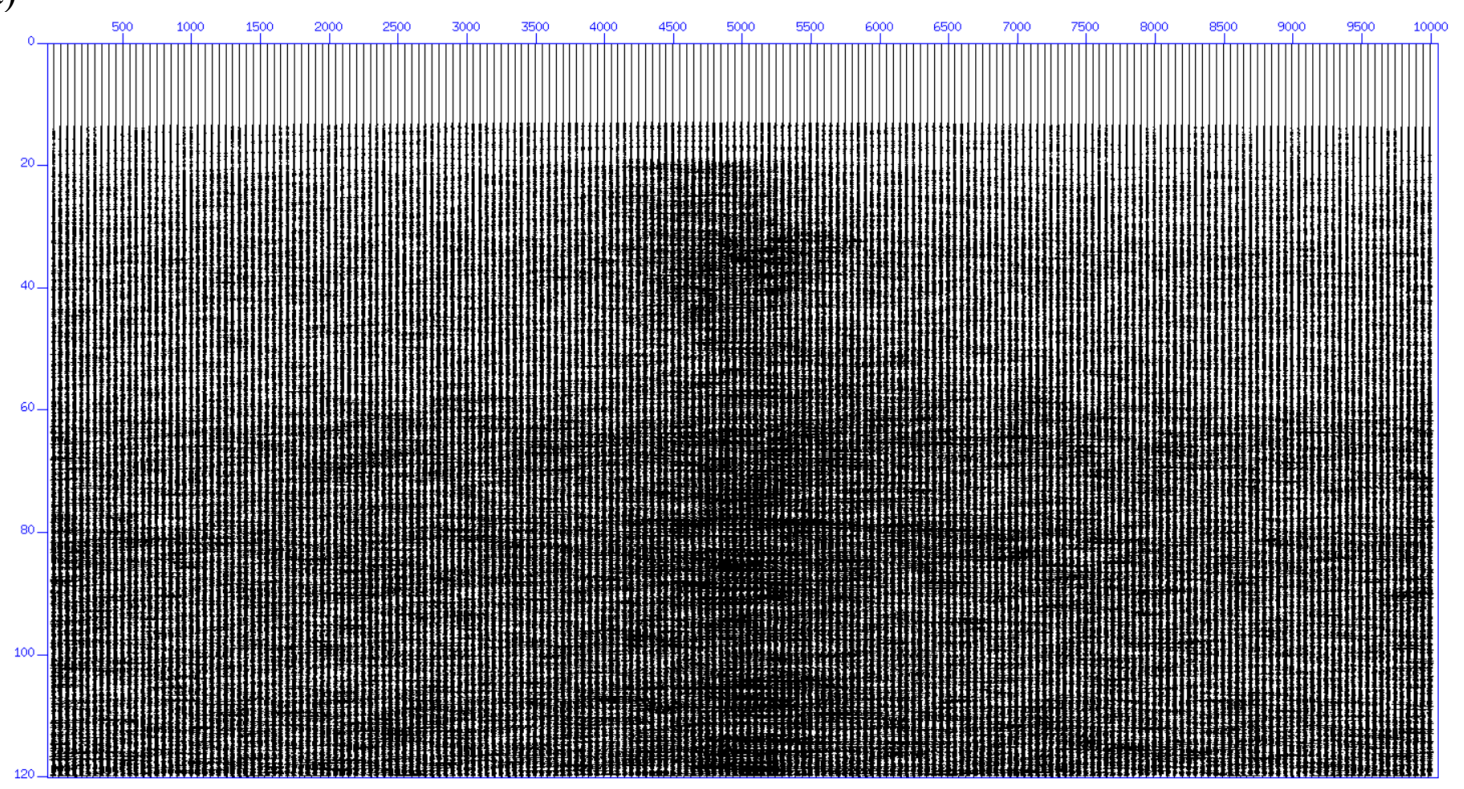


(b)

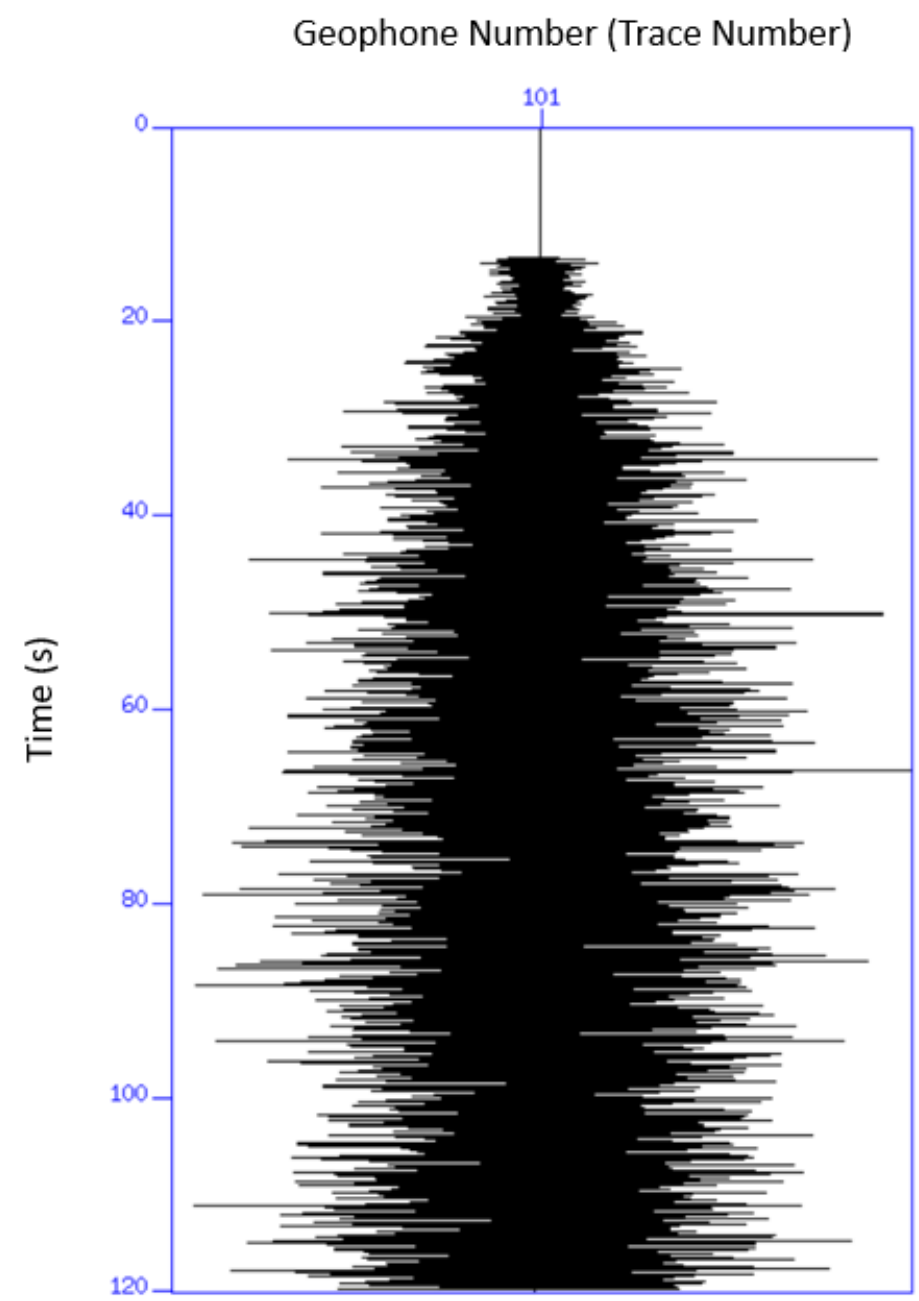


(c)

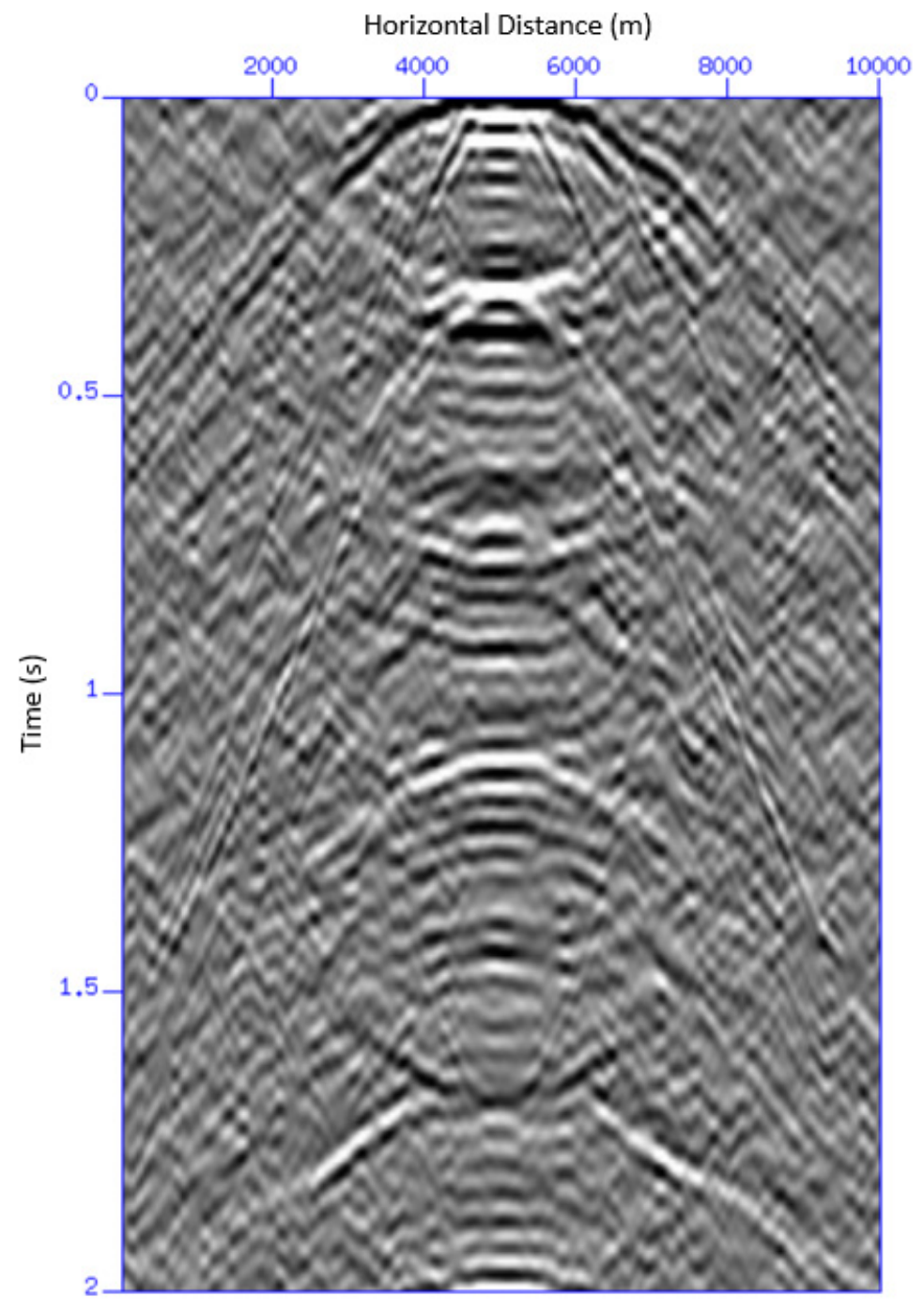

Figure 11. (a) The $120 \mathrm{~s}$ long acoustic transmission response (noisy data). (b) The master trace 101 among all 201 traces. (c) The reconstructed reflection response or virtual shot gather as if from a source at $5,000 \mathrm{~m}$. 
The same procedure is applied to the zero-offset VSP method. After creating a noise panel, the RMS normalization is performed to avoid amplitude changes. Then, the cross correlation is applied to the normalized noisy VSP data as a pre-processing. In the zerooffset VSP method, geophones are vertically located inside the borehole. While performing the cross-correlation process, the first geophone inside the borehole is selected as the master trace and is cross-correlated with all other traces. After, the second geophone is selected, and the same process is applied. In this way, the cross-correlation process is applied until all geophones inside the borehole are completed (Figure 12).
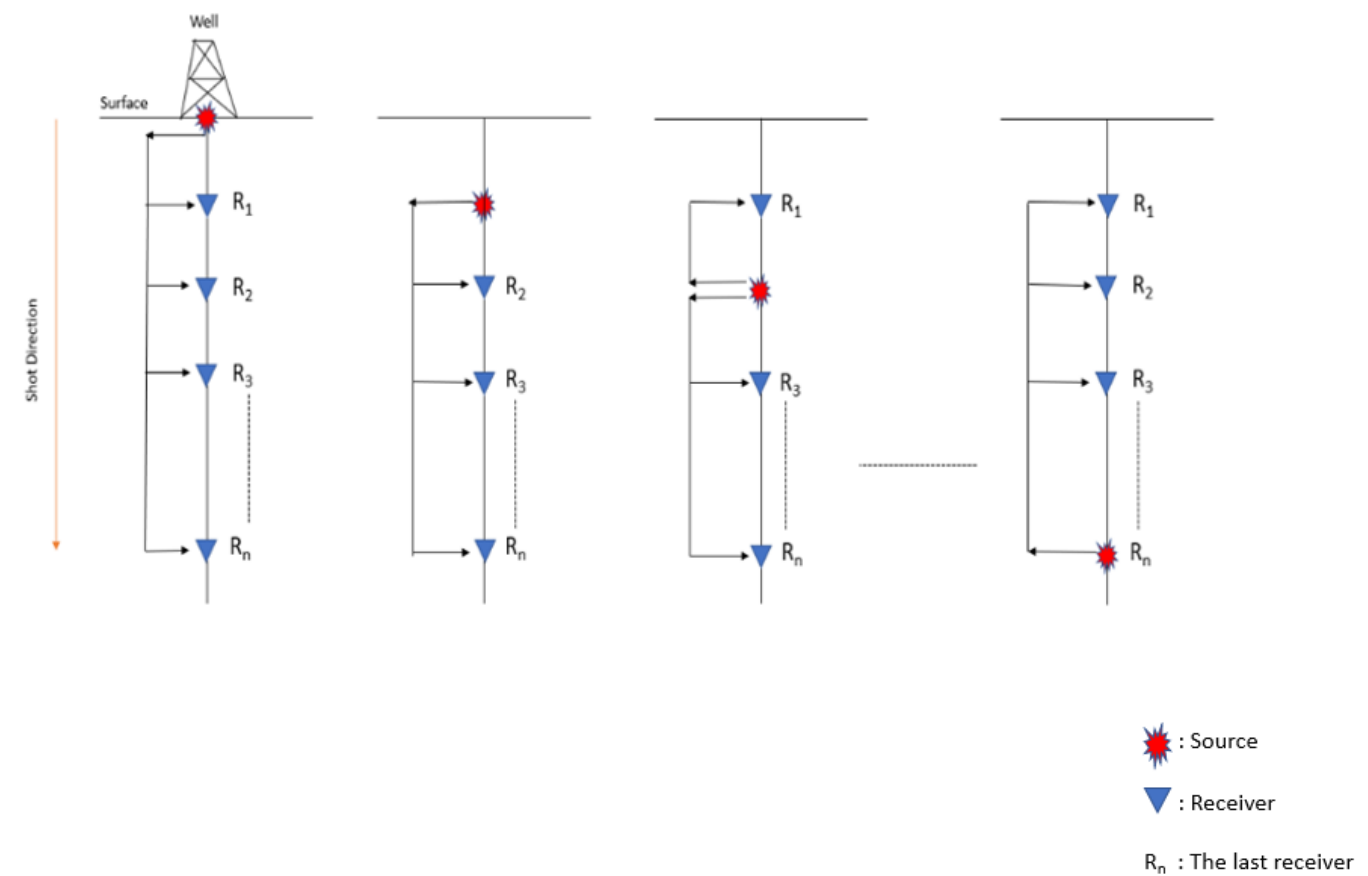

Figure 12. The cross-correlation procedure for the zero-offset VSP interferometry. This figure was generated utilizing Matsushima (2016).

To show how a VSP virtual shot produced, again, I utilized 20 sources inside boundary 1 in a flat VSP model to obtain a raw noise panel by the borehole geophone (Figure 13a). From this data, a master trace is selected (trace 4 out of the 101, Figure 13b). Then, the master trace is cross-correlated with other traces in the borehole to a virtual VSP shot gather is obtained (Figure 13c). 
(a)

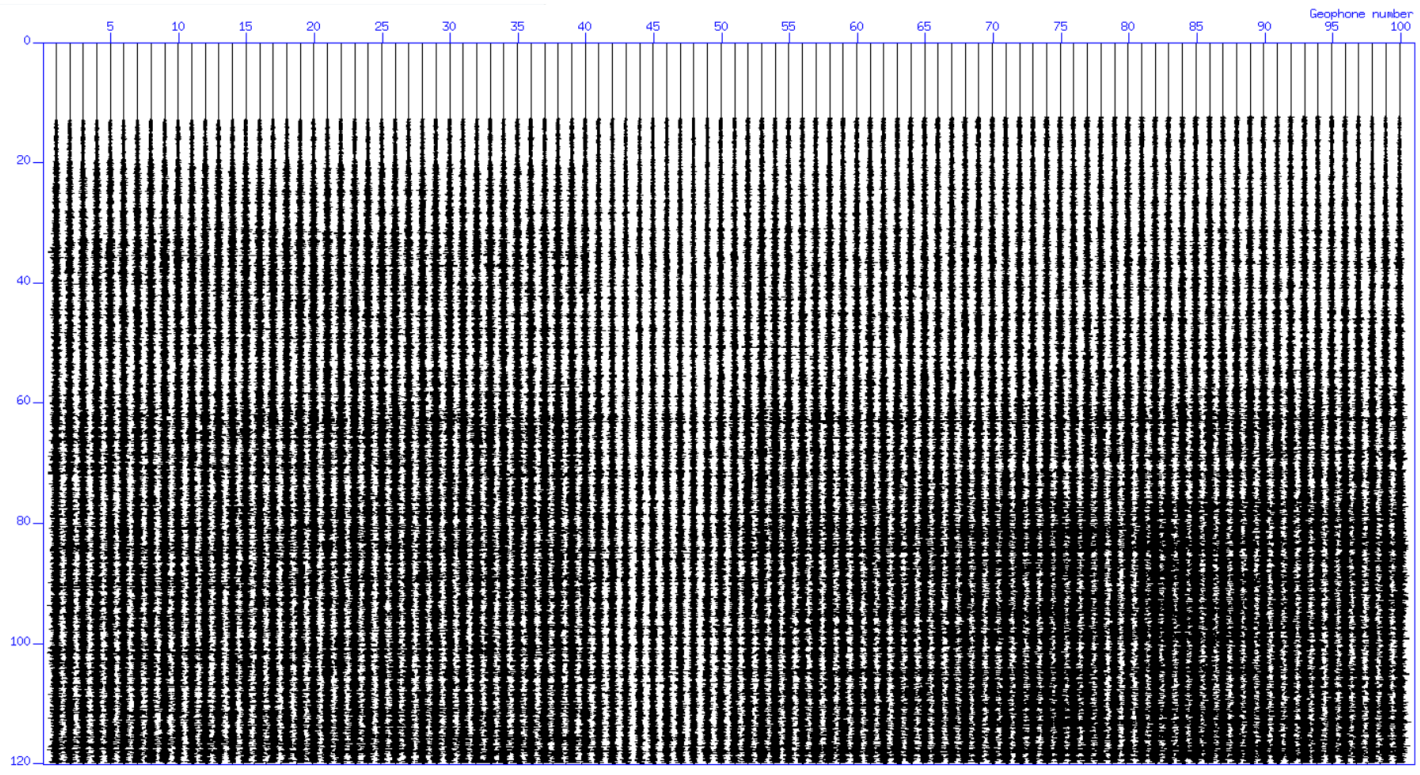


(b)

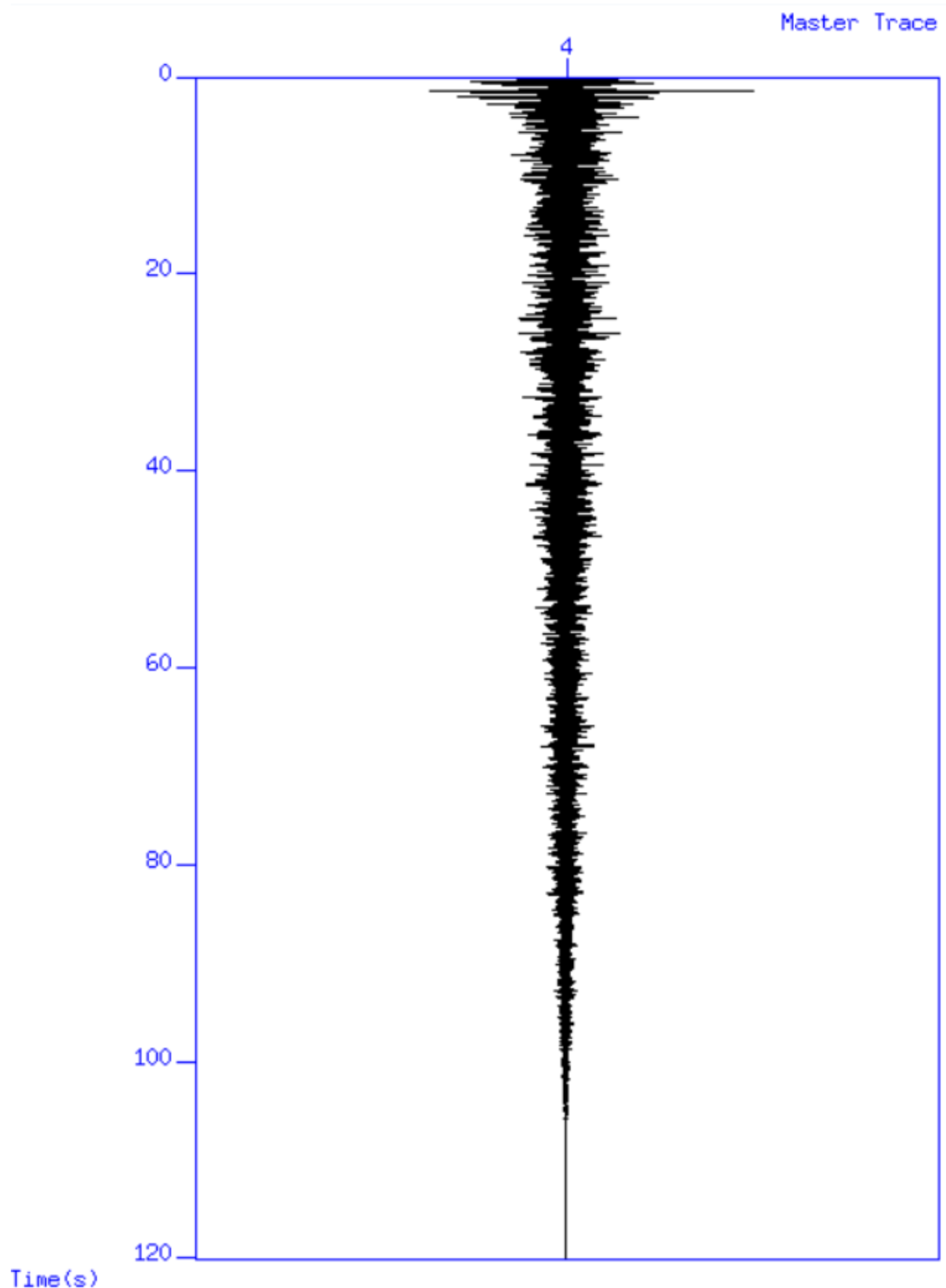


(c)

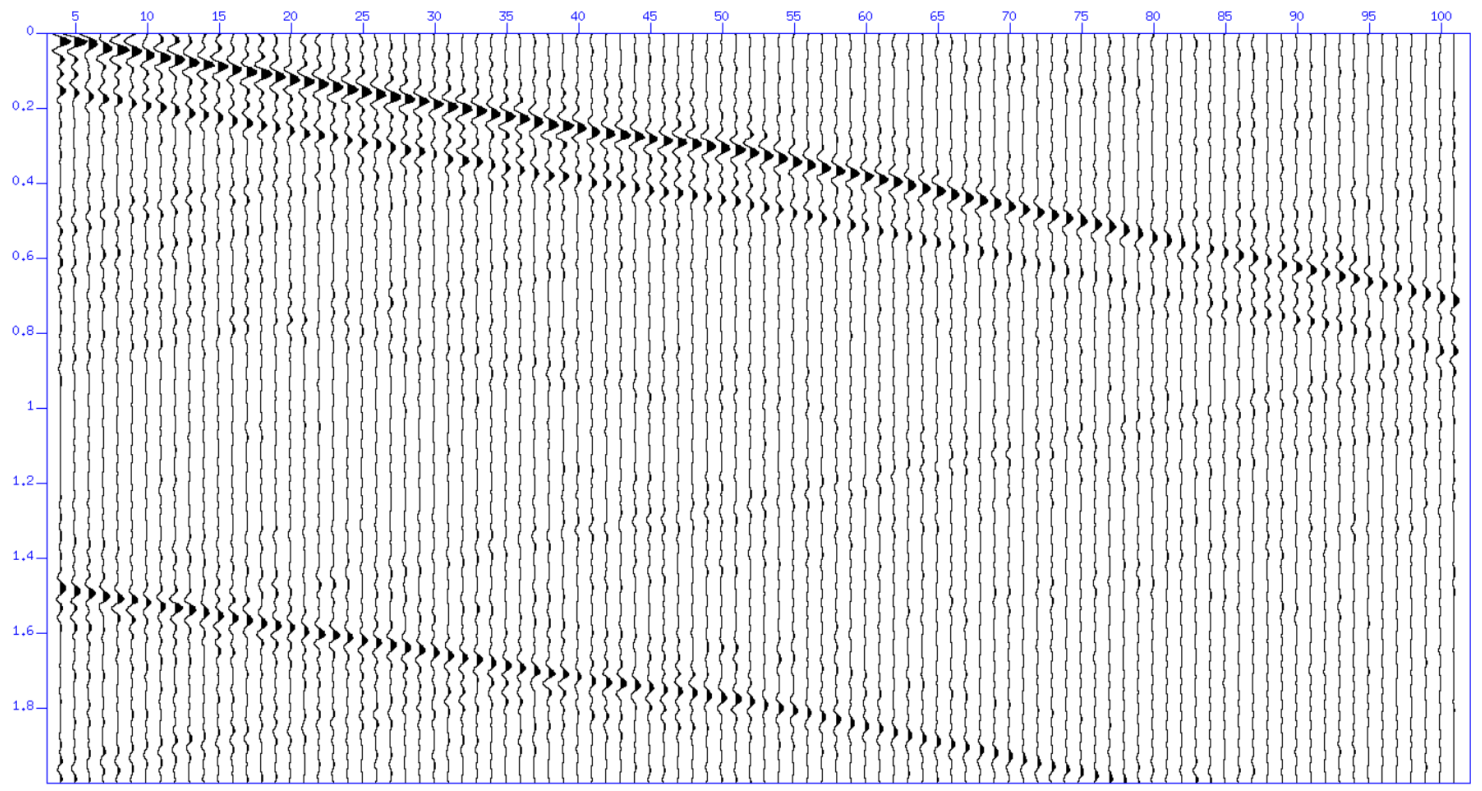

Figure 13. The retrieving VSP reflection response from noisy VSP data by crosscorrelation method. (a) shows uncorrelated noisy VSP data. (b) shows the first trace used in the cross-correlation as a master trace. (c) shows the virtual VSP shot gather after crosscorrelation method. 


\section{Processing Details}

\subsection{Surface Seismic Geometry}

Using the 2D finite-difference wavefield modeling (fdelmodc) code, 2D velocity models have been created. Randomly distributed uncorrelated underground noise sources at a depth between $100 \mathrm{~m}$ and 3,900 m were recorded for 120 seconds, and by recording these sources by geophones, noisy data or known as acoustic transmission responses were obtained. Figure 14 shows the noisy or raw data, which is not correlated, of boundary 1 that contains 20 sources in the anticline model as an example.

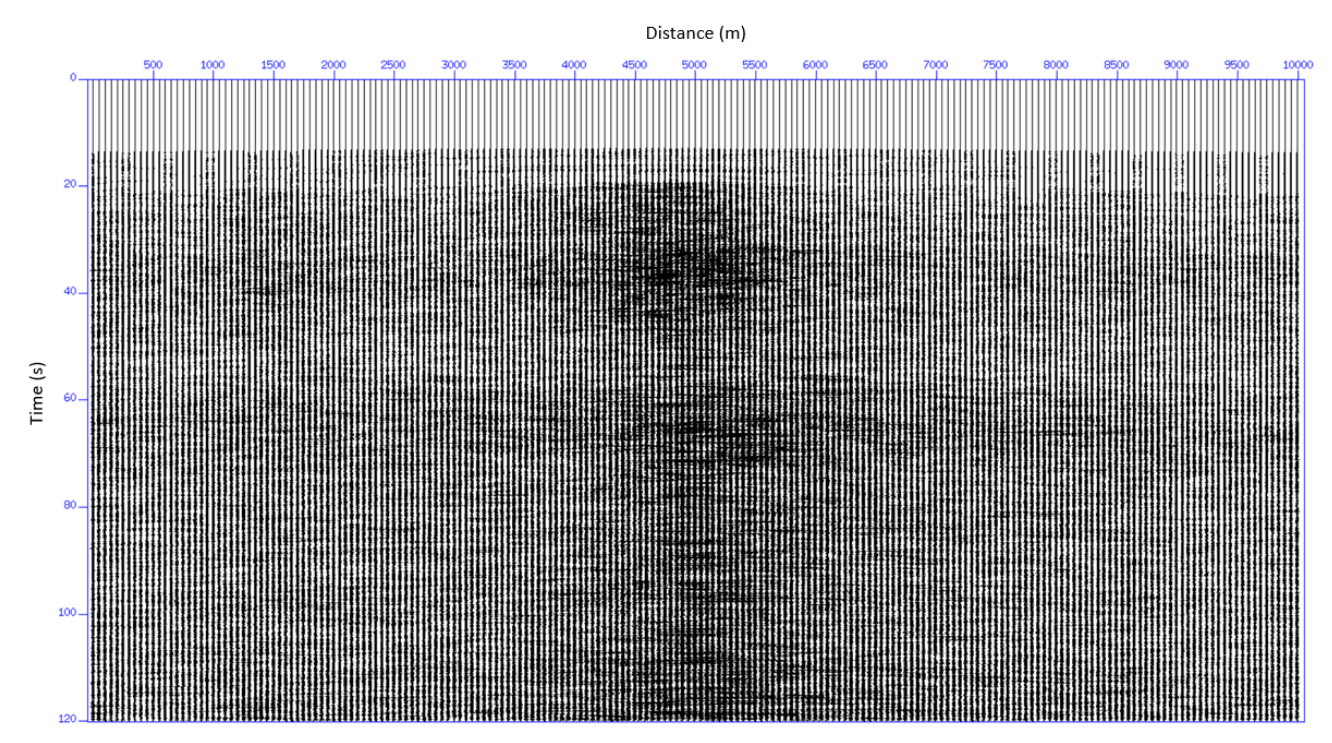

Figure 14. A raw data of 120 seconds long of boundary 1 for 20 sources. The noise sources starting from $100 \mathrm{~m}$ to $3,900 \mathrm{~m}$ depth and extend from 4,500 m-5,500 m lateral distance.

In Figure 14, we note uneven amplitudes. To make the cross-correlation contributions of all traces equal, RMS normalization is applied. Then, cross-correlation is applied to obtain virtual shot gather. In surface seismic geometry, 101 out of a total of 201 geophones, that is, the geophone in the middle was used as the master trace and this was cross-correlated with all the other remaining traces. The virtual shot gathers, as known as reconstructed reflection response, obtained as a result of cross-correlation is compared corresponding to the different noise boundaries in Figures 15 (flat-layered model, Figures 16 (anticline model) and Figures 17 (anticline-syncline model) for 5, 20 sources, and 500 sources. In Figure 15, the reflection hyperbolas corresponding to the 4 flat layers in the flat-layered velocity model are seen at around $0.53 \mathrm{~s}, 1.03 \mathrm{~s}, 1.19 \mathrm{~s}$, and $1.79 \mathrm{~s}$ (yellow). Reflection hyperbolas corresponding to the four anticlines in the anticline model are seen at around $0.33 \mathrm{~s}, 0.83 \mathrm{~s}, 1.11 \mathrm{~s}, 1.63 \mathrm{~s}$ (yellow arrows in Figure 16). In Figure 17, the reflection hyperbolas corresponding to the 3 anticlines and 1 syncline in the anticline-syncline model are seen at around $0.2 \mathrm{~s}, 0.65 \mathrm{~s}, 0.81 \mathrm{~s}$, and 1.91s (yellow arrows in Figure 17). 
(a)
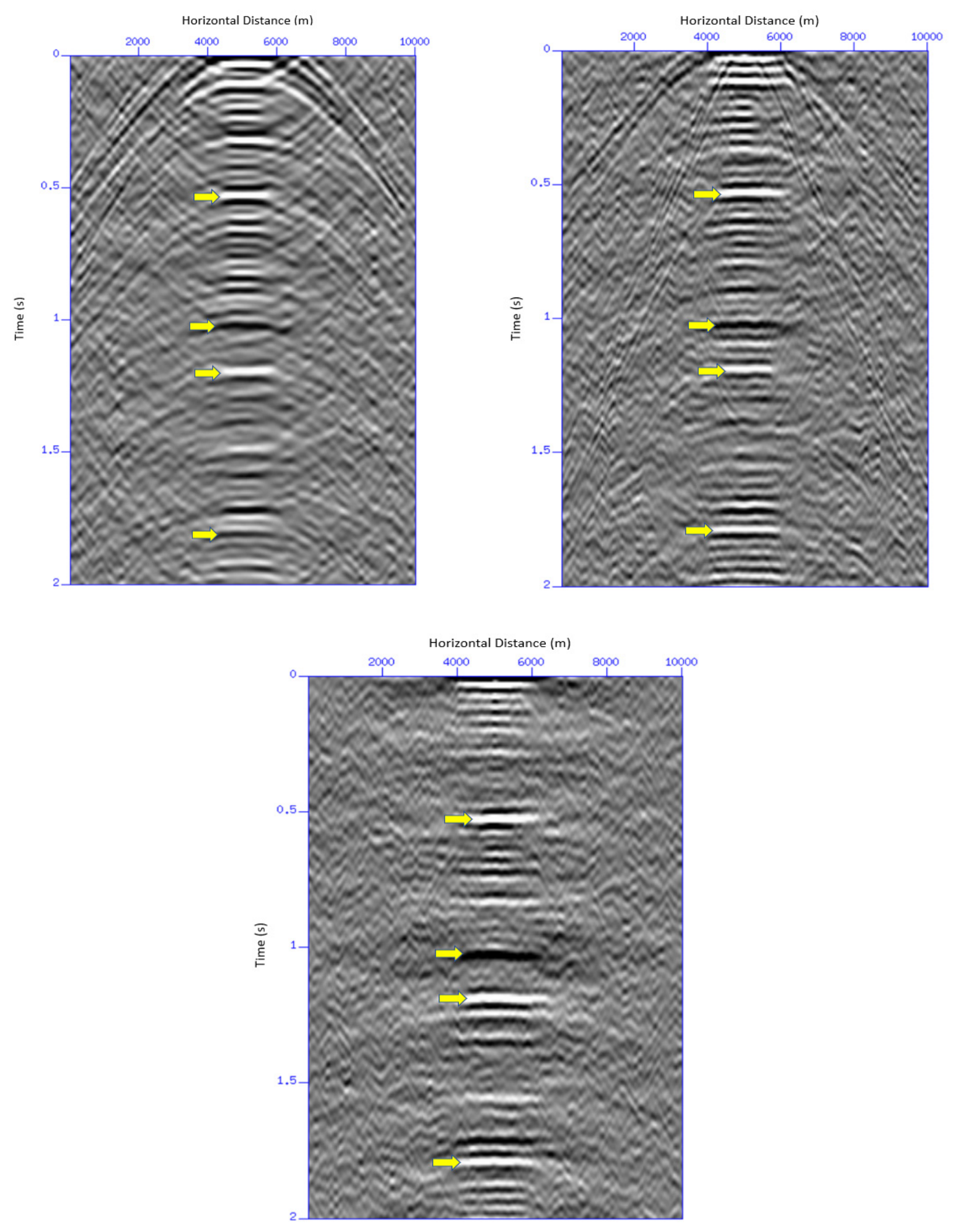
(b)
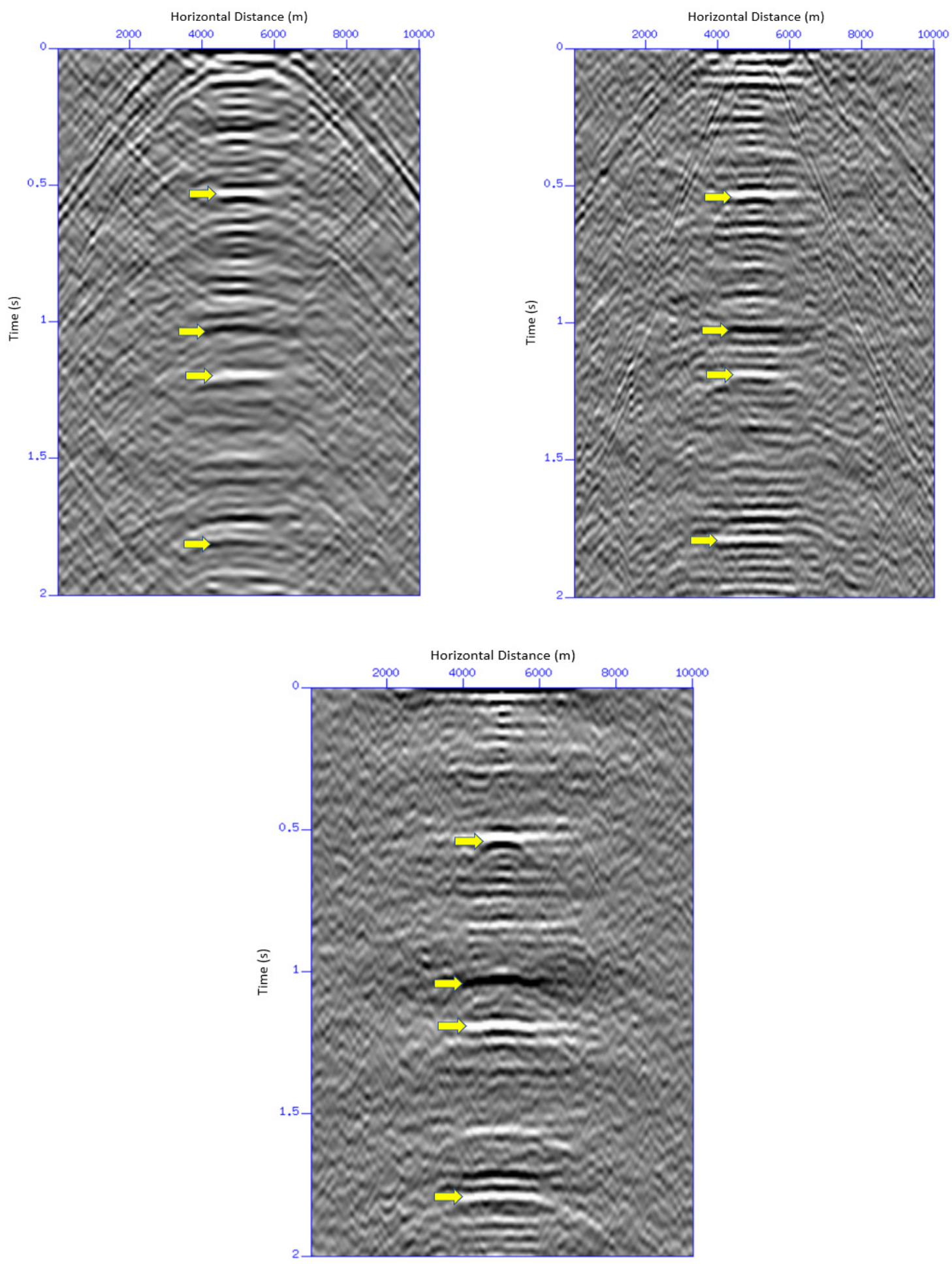
(c)
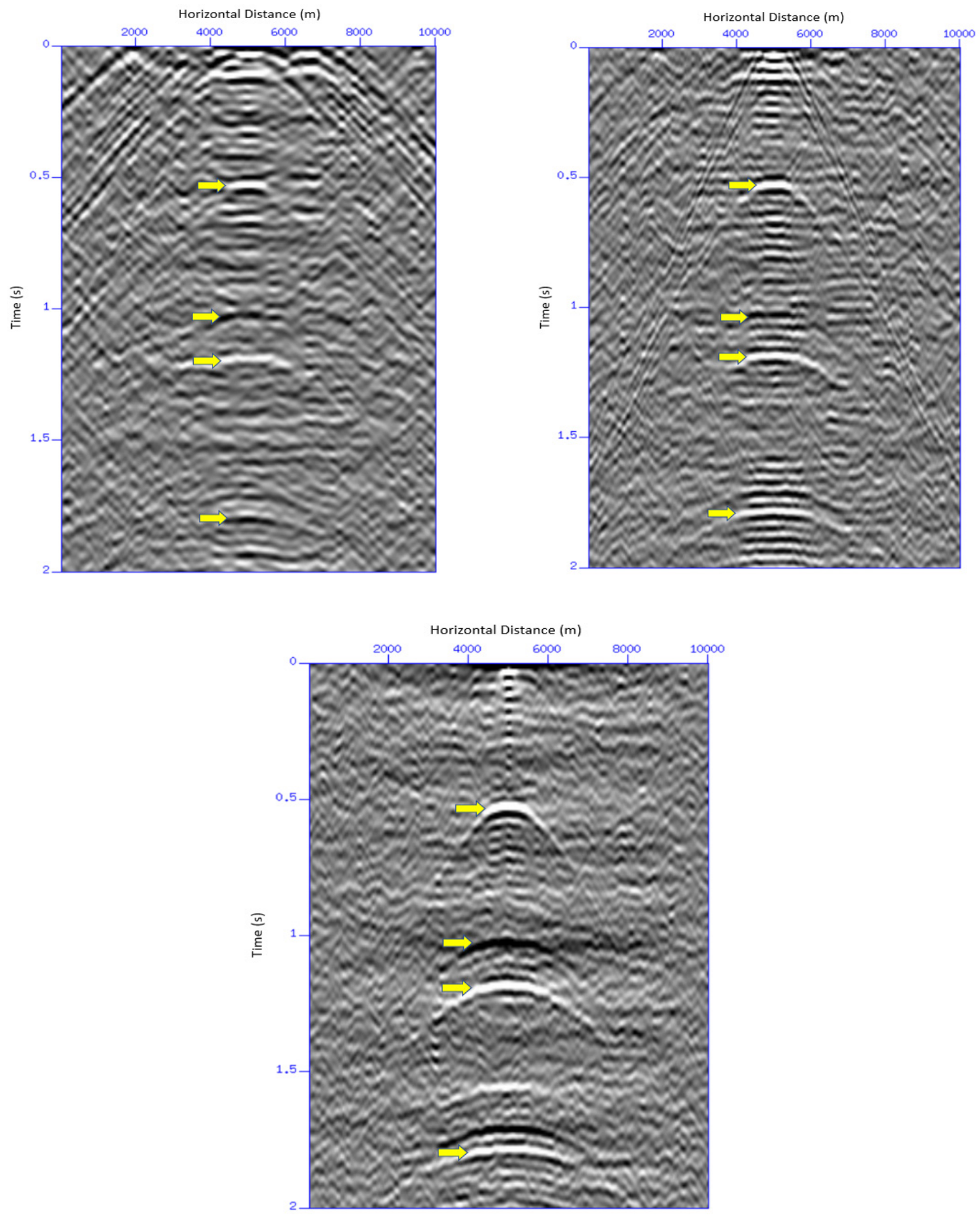
(d)
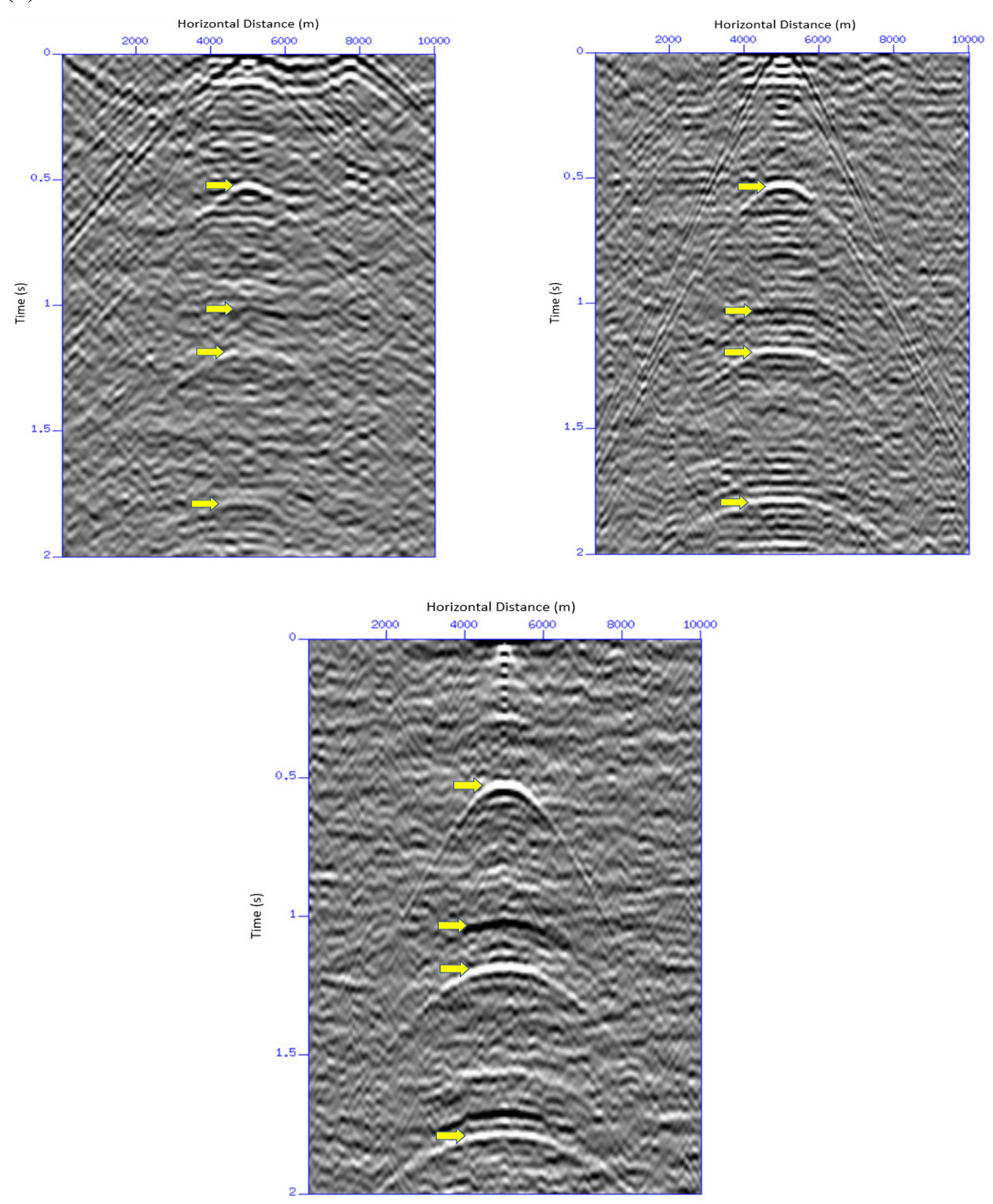

Figure 15. The comparison of virtual shot gathers of 5 sources (left-hand side), 20 sources (right-hand side), and 500 sources (bottom) for boundary 1, 2, 3, and 4 in Figure 15a, Figure $15 \mathrm{~b}$, Figure 15c, and Figure 15d in the flat model, respectively. The yellow arrows correspond to the reflections from four interfaces. 
(a)
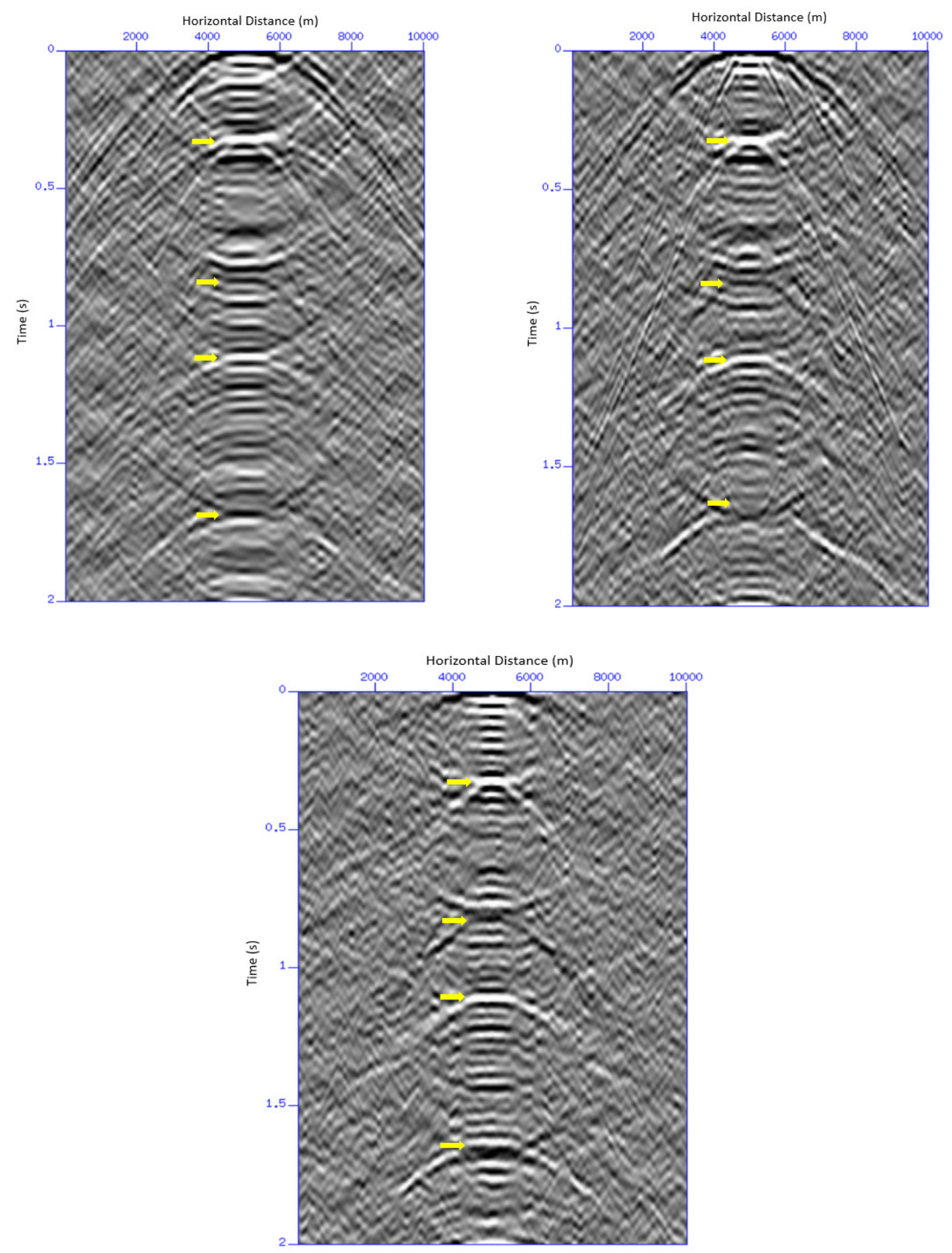
(b)
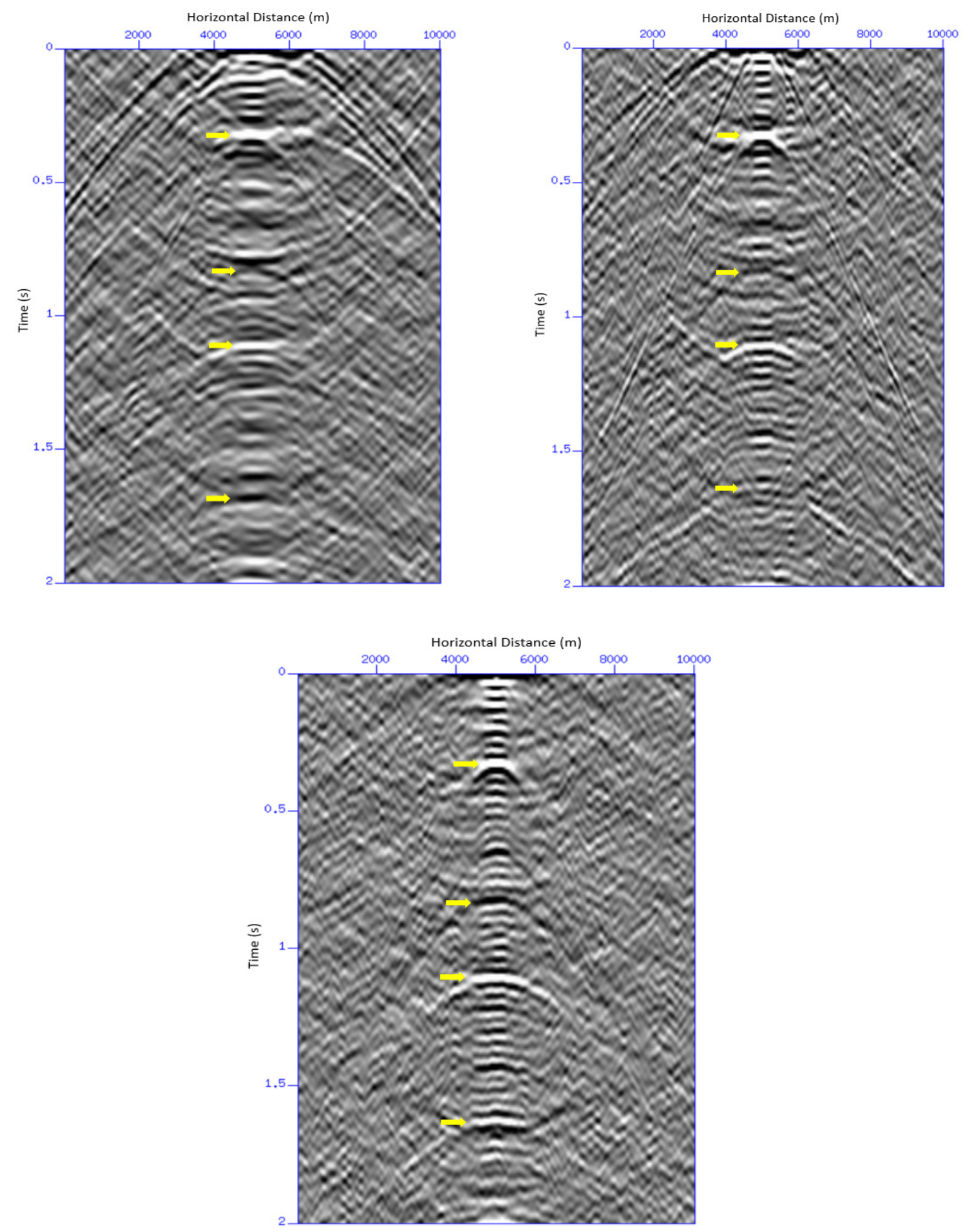
(c)
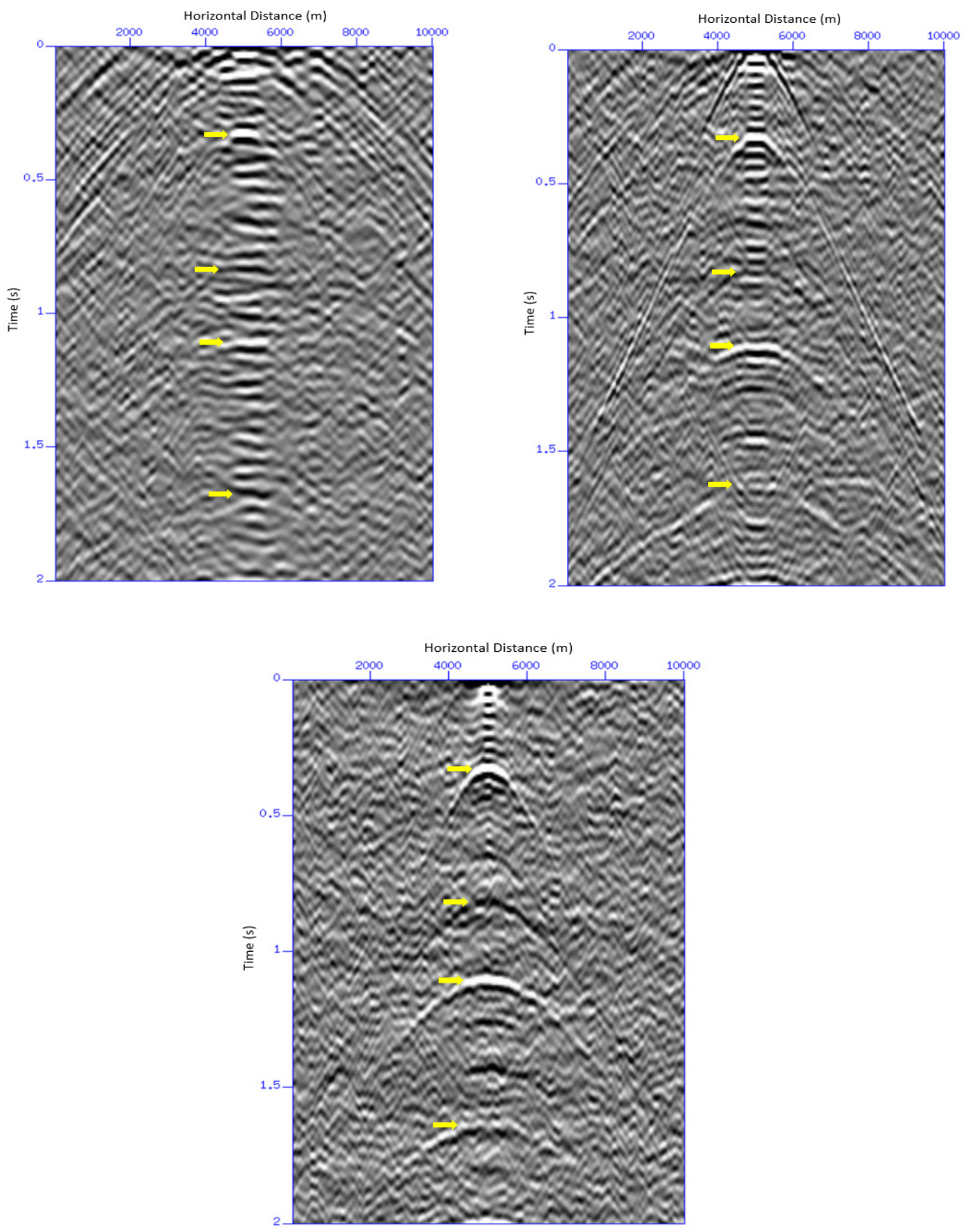

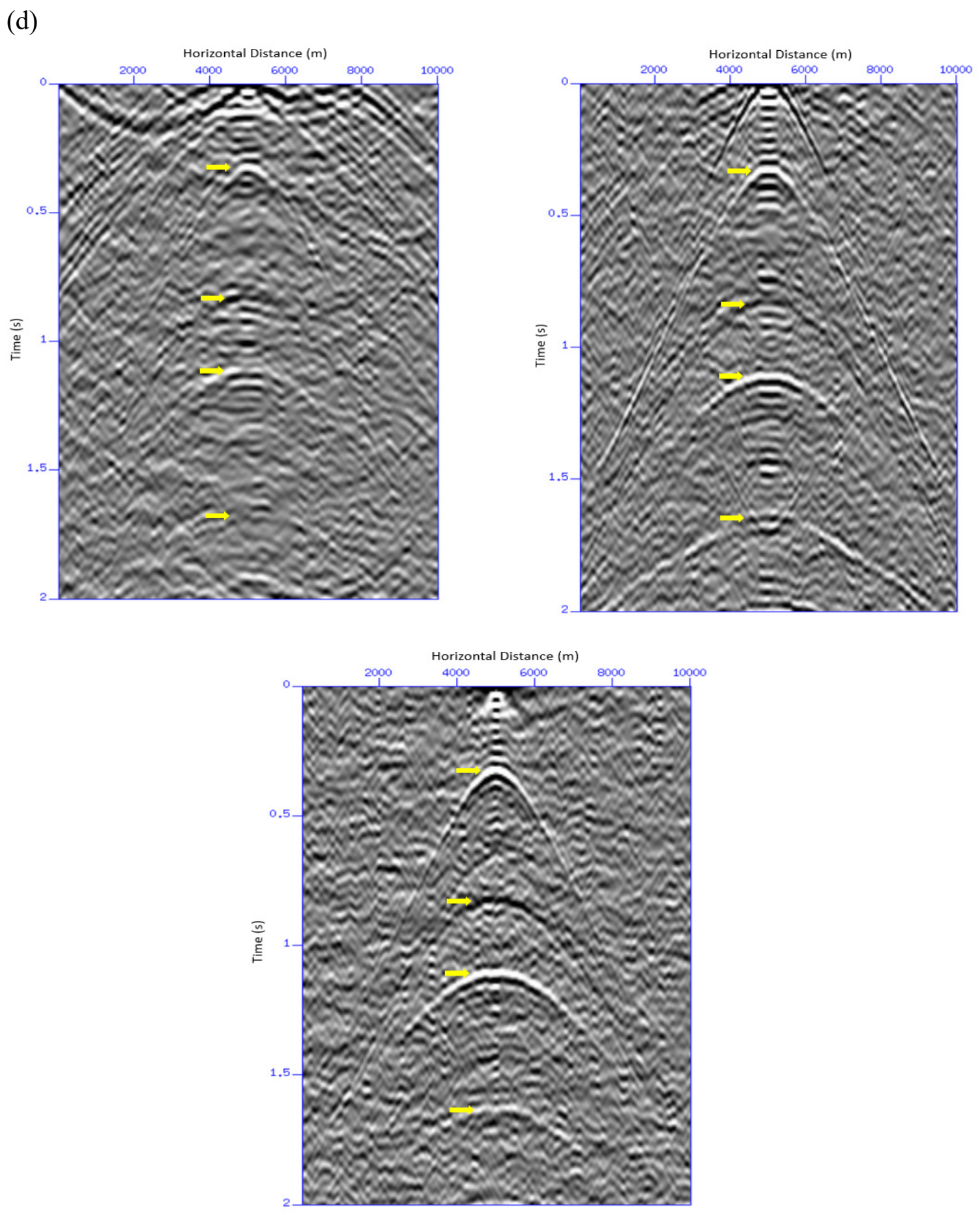

Figure 16. The comparison of virtual shot gathers of 5 sources (left-hand side), 20 sources (right-hand side), and 500 sources (bottom) for boundary 1,2, 3, and 4 in Figure 16a, Figure 16b, Figure 16c, and Figure 16d in the anticline model, respectively. The yellow arrows correspond to the reflections from four interfaces. 
(a)
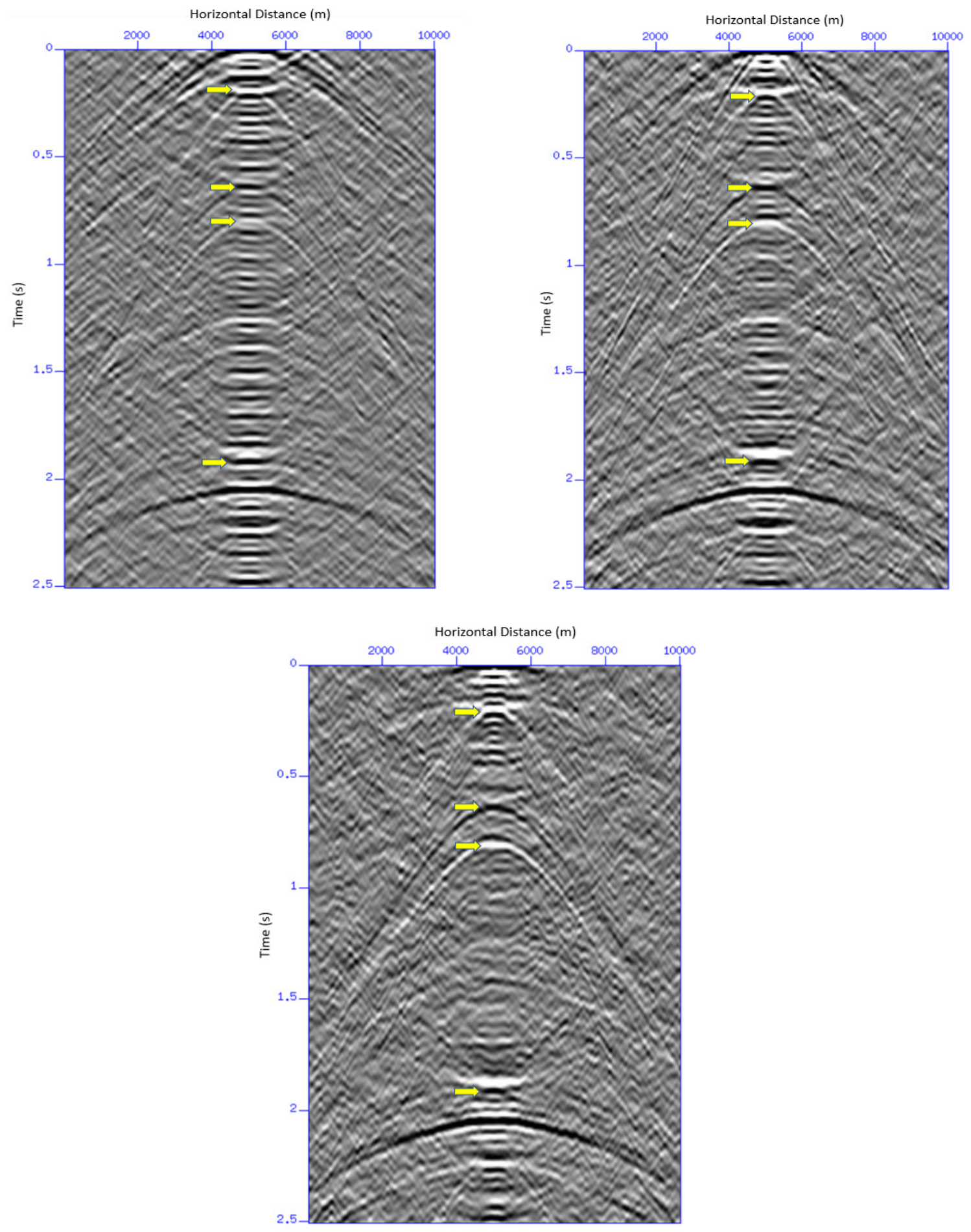
(b)
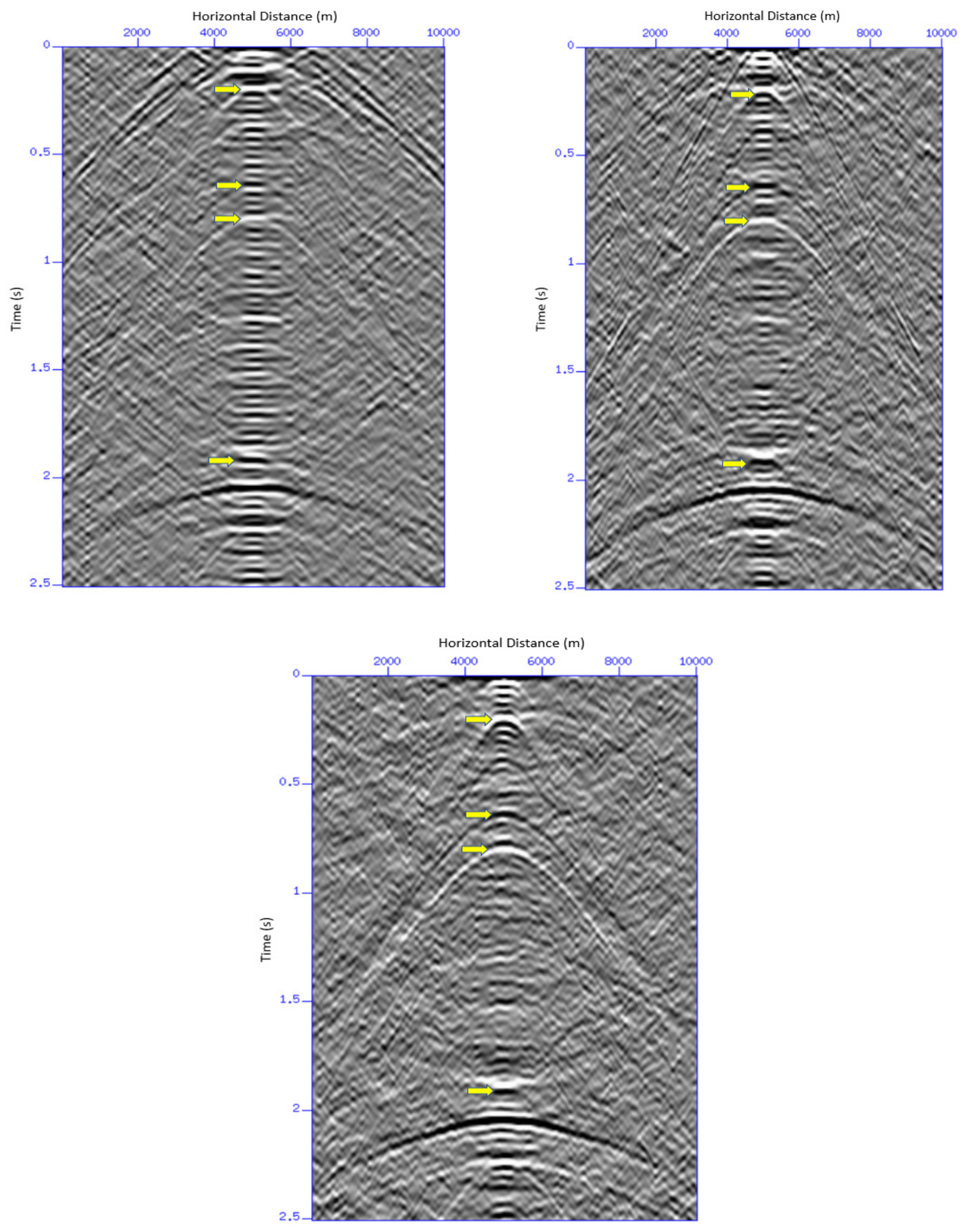
(c)
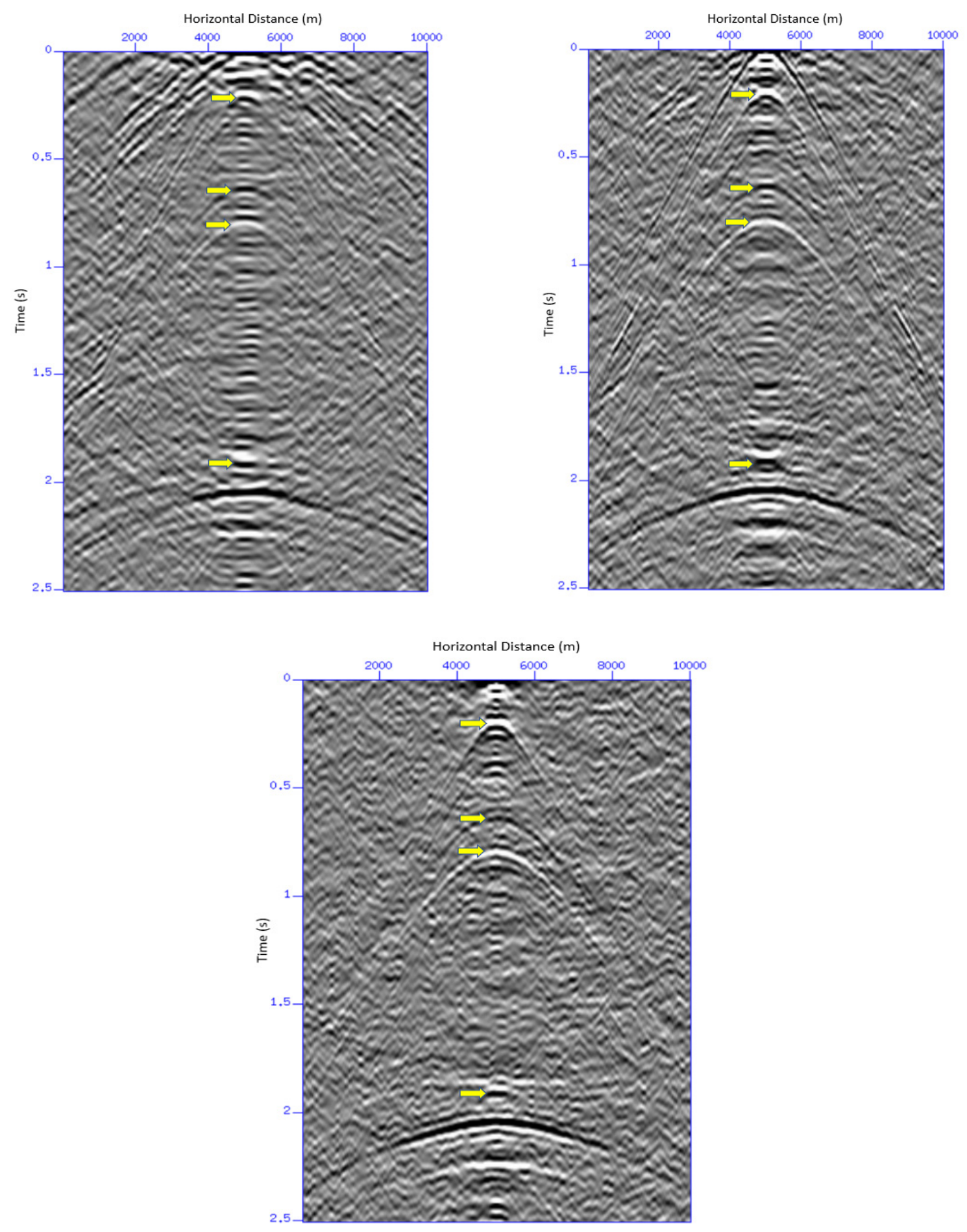
(d)
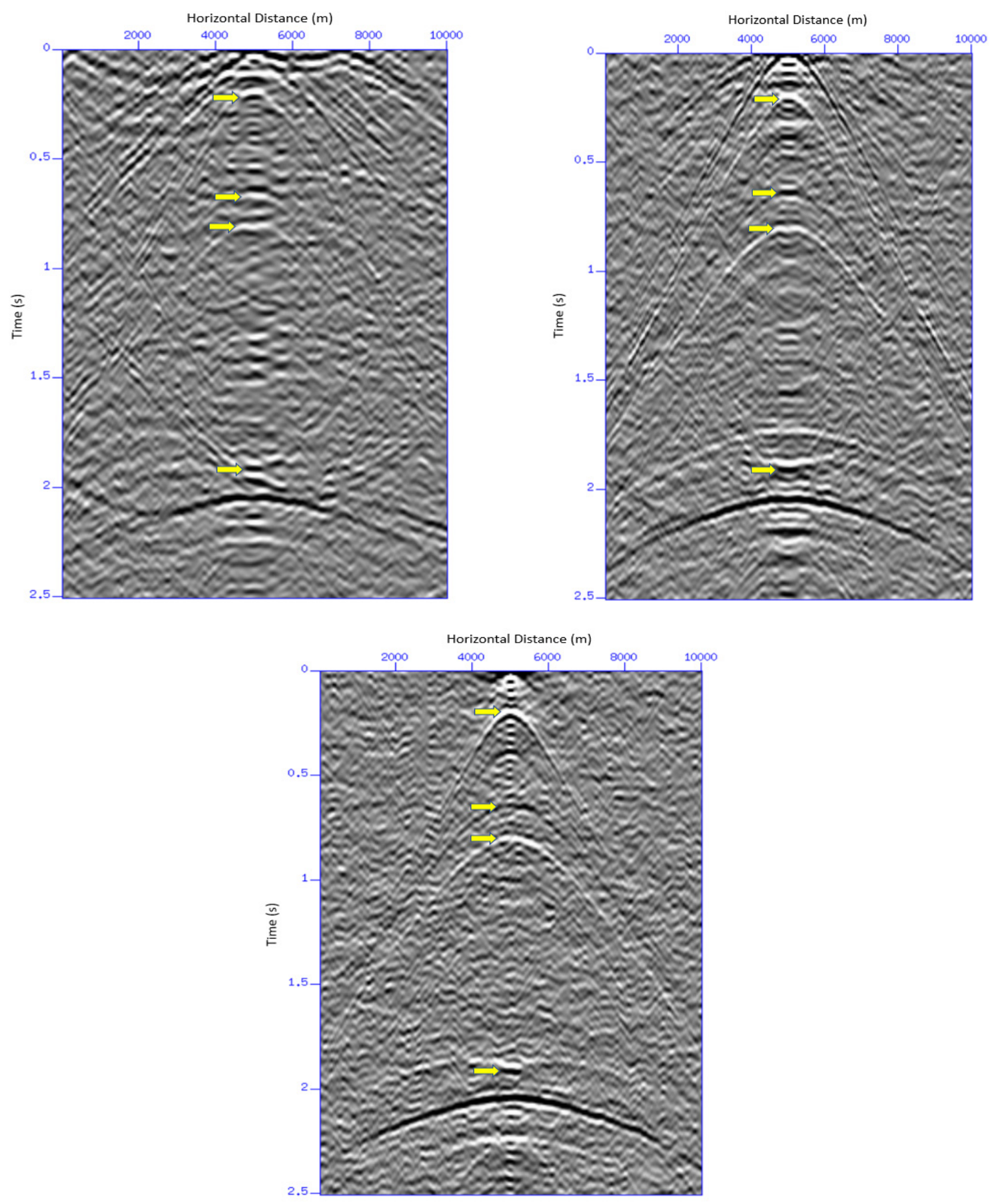

Figure 17. The comparison of virtual shot gathers of 5 sources (left-hand side), 20 sources (right-hand side), and 500 sources (bottom) for boundary 1, 2, 3, and 4 in Figure 17a, Figure $17 \mathrm{~b}$, Figure 17c, and Figure 17d in the anticline-syncline model, respectively. The yellow arrows correspond to the reflections from four interfaces. 
After obtaining virtual shot gathers, they were sorted into common midpoint (CMP) gathers. Then, a velocity analysis was performed on a selected group of CMP gather. Figure 18 shows CMP gather 2000 for 500 sources boundary 1 for flat model regarding velocity analysis as an example. As a result of the velocity analysis, reflections showing hyperbolic behavior in CMP gathers were flattened by NMO correction and all wavelets were lined up to zero-offset times.

By stacking after NMO correction, the ambient noise effect in the data is removed and the signal to noise ratio of the seismic data is enhanced. In other words, quality is increased. By repeating this process for all selected groups of CMP gathers in seismic data, a zero offset stacked image is obtained, which is the image of the model that was initially created with the fdelmode program.

Figures from 19 to 27 show unmigrated zero-offset figures for four boundaries about 5, 20 sources, and 500 sources for flat, anticline and anticline-syncline models, respectively. In these figures, the $\mathrm{x}$-axis shows offset (m), and the y-axis shows the time (s). As a result, underground structures have been successfully retrieved by surface seismic interferometry. 
(a) Before Velocity Picking
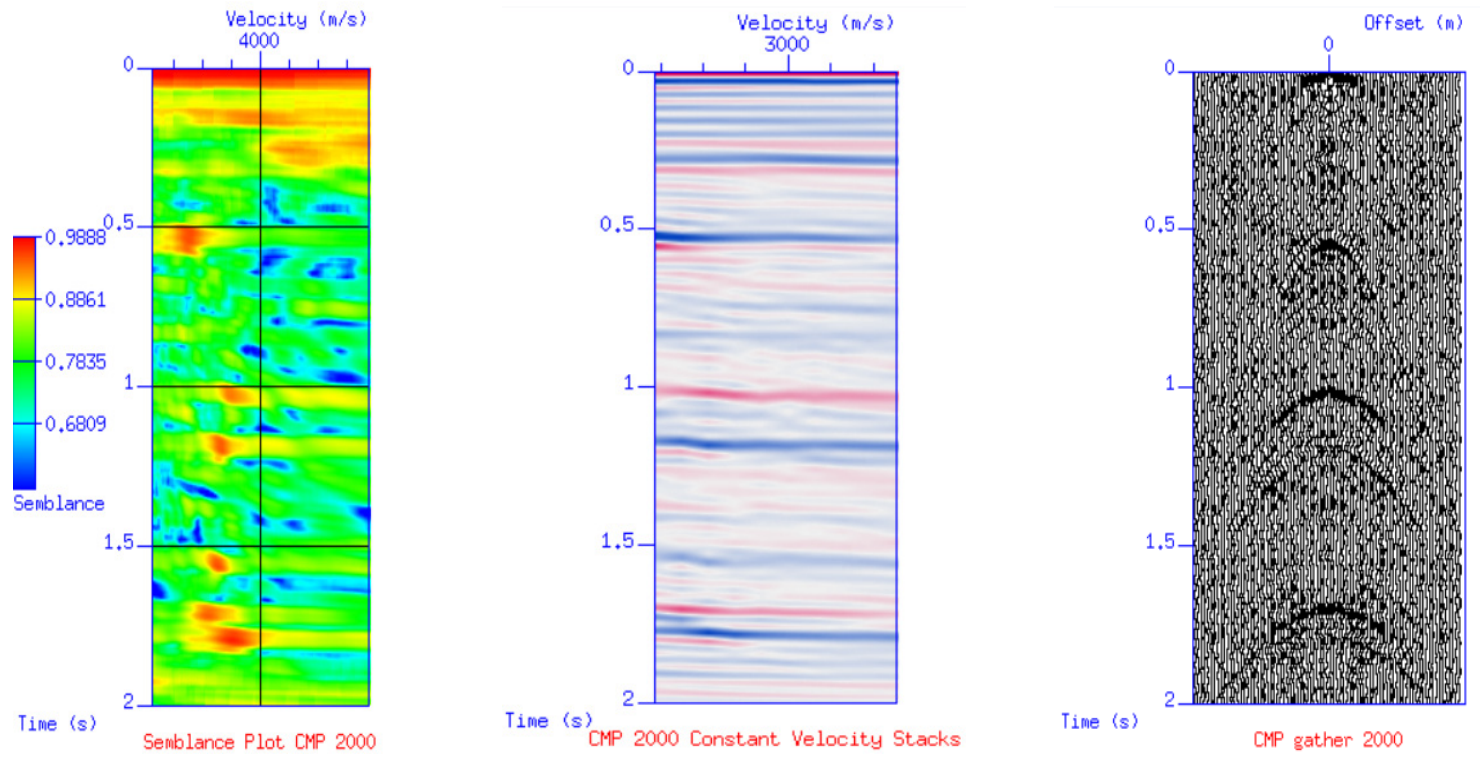

CMP gather 2000

(b) After Velocity Picking

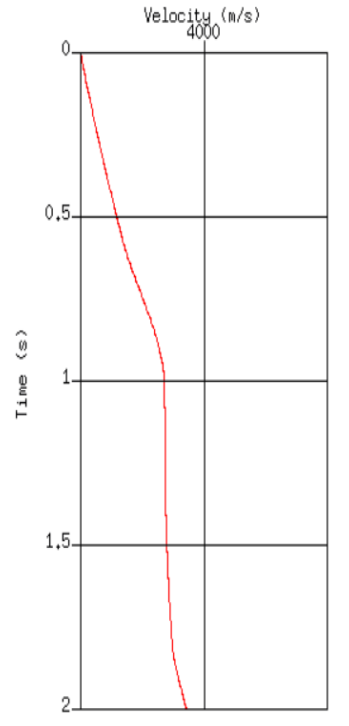

CMP 2000 Stacking Velocity Function

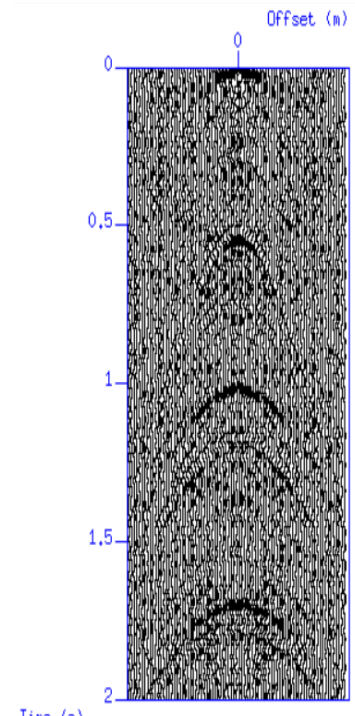

CMP gather 2000

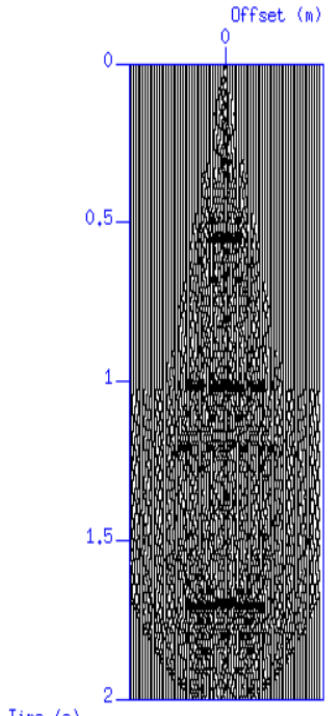

CMP 2000 after NMO

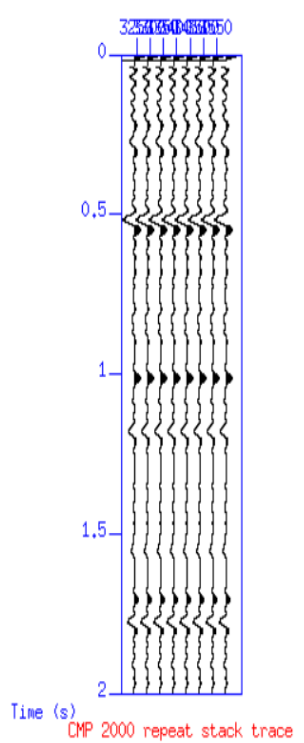

Time $\langle s\rangle$ CMP 2000 repeat stack trace

Figure 18. (a) Before velocity picking of CMP gather 2000 for 500 sources boundary 1. (b) After velocity picking of CMP gather 2000 for 500 sources boundary 1 in the flat model as an example. 

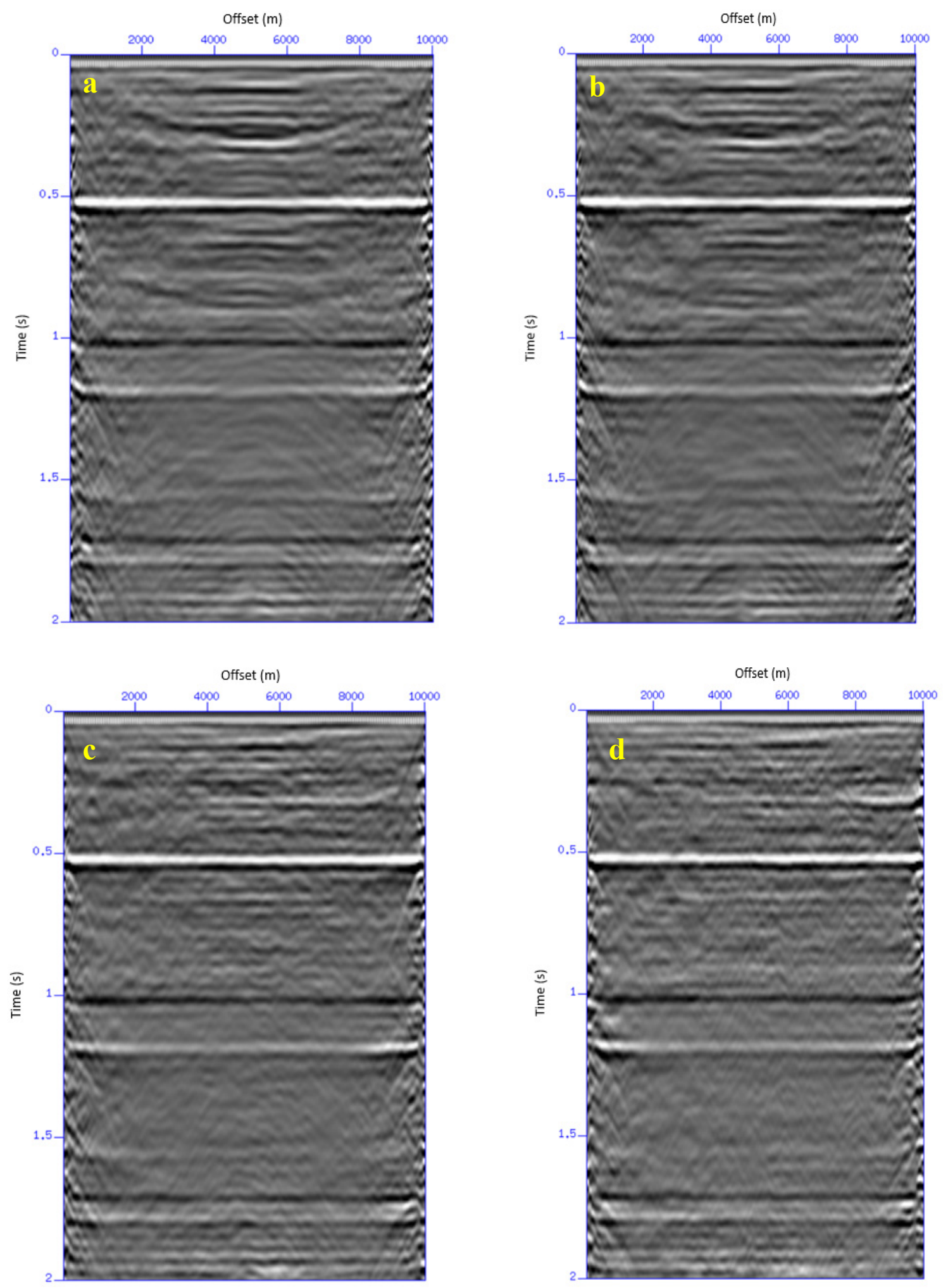

Figure 19. (a), (b), (c) and (d) shows the unmigrated zero-offset sections obtained from boundaries 1, 2, 3, and 4 for 5 sources about the flat model, respectively. 

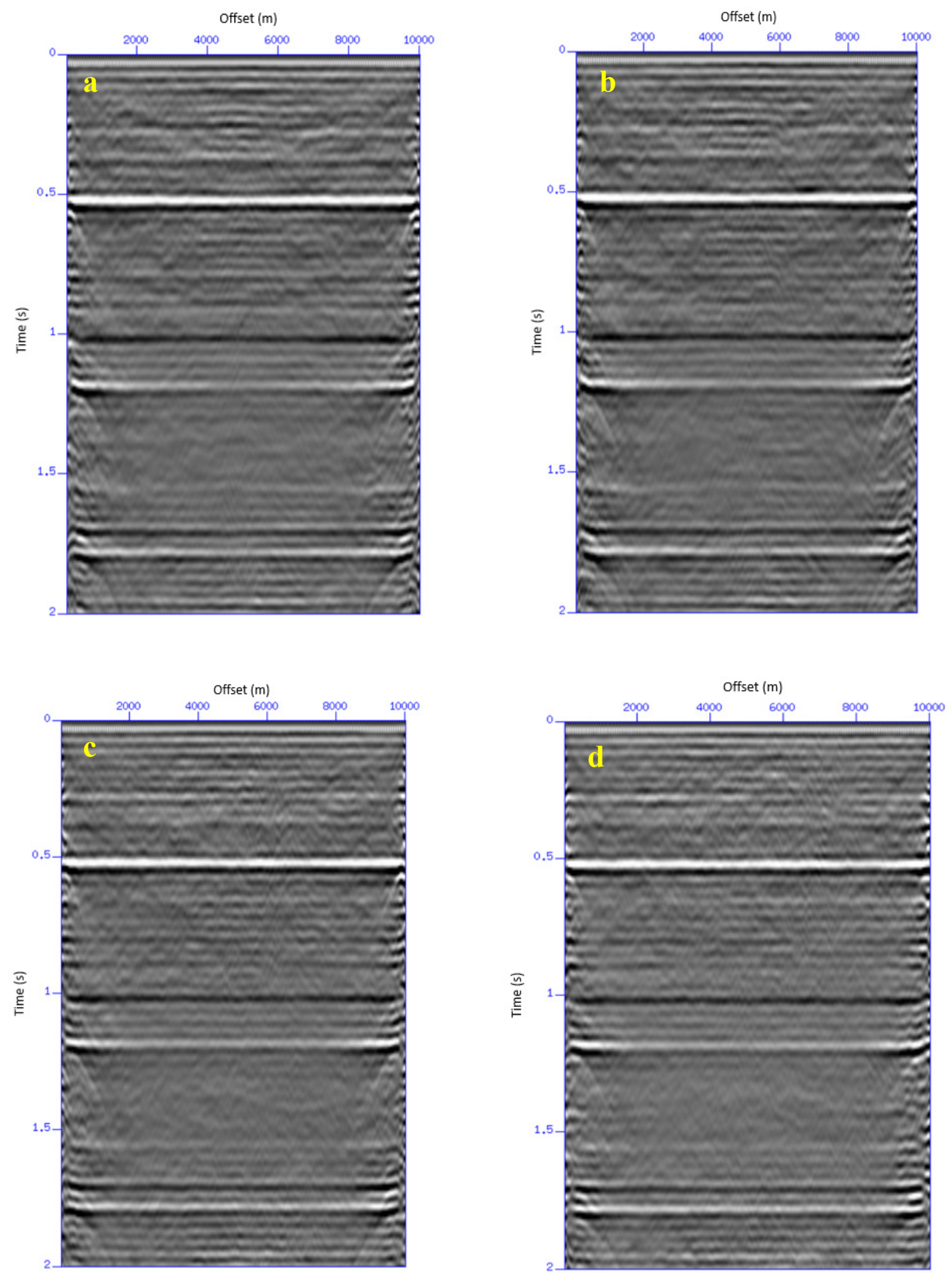

Figure 20. (a), (b), (c) and (d) shows the unmigrated zero-offset sections obtained from boundaries 1, 2, 3, and 4 for 20 sources about the flat model, respectively. 

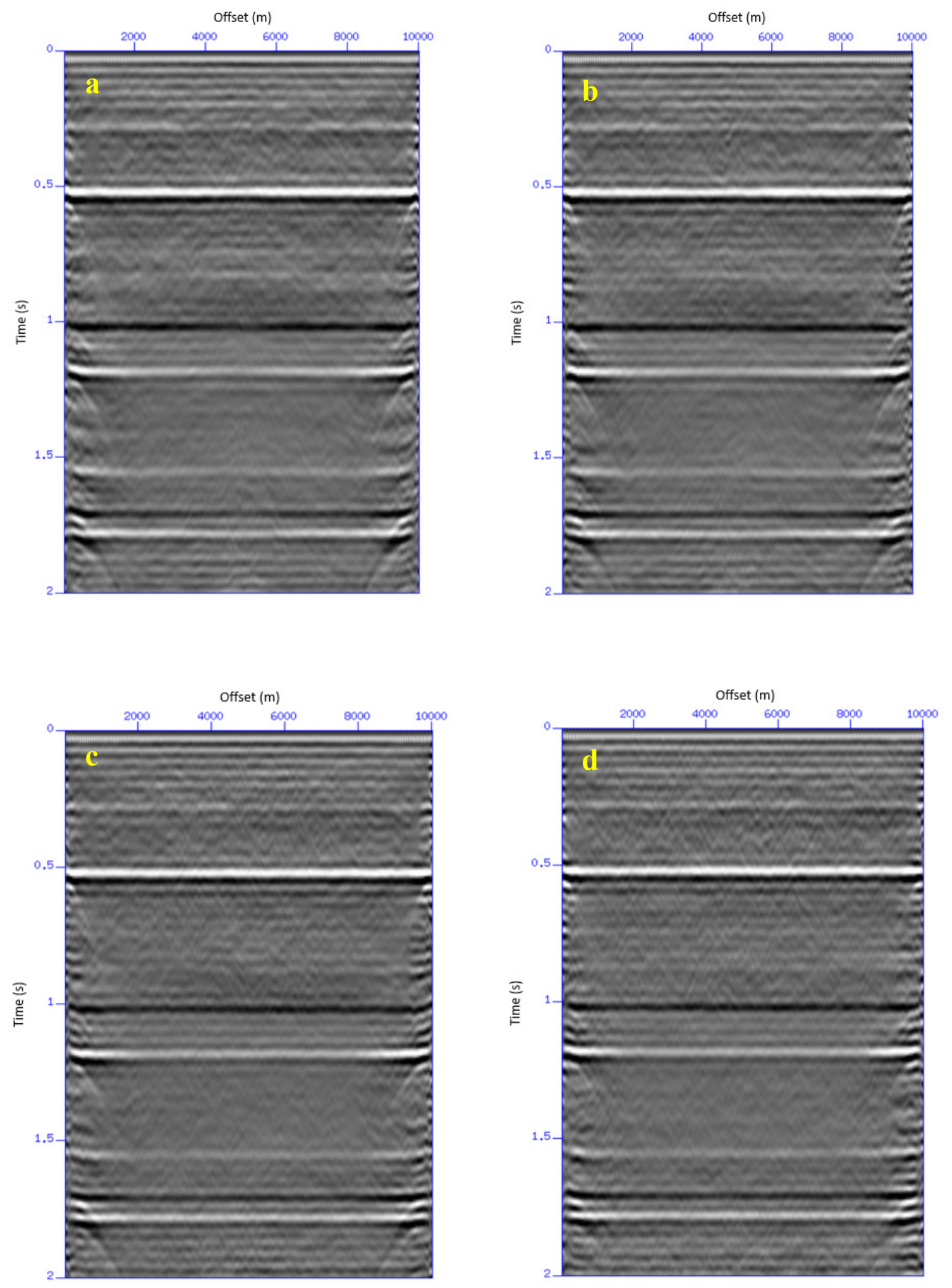

Figure 21. (a), (b), (c) and (d) shows the unmigrated zero-offset sections obtained from boundaries 1, 2, 3, and 4 for 500 sources about the flat model, respectively. 

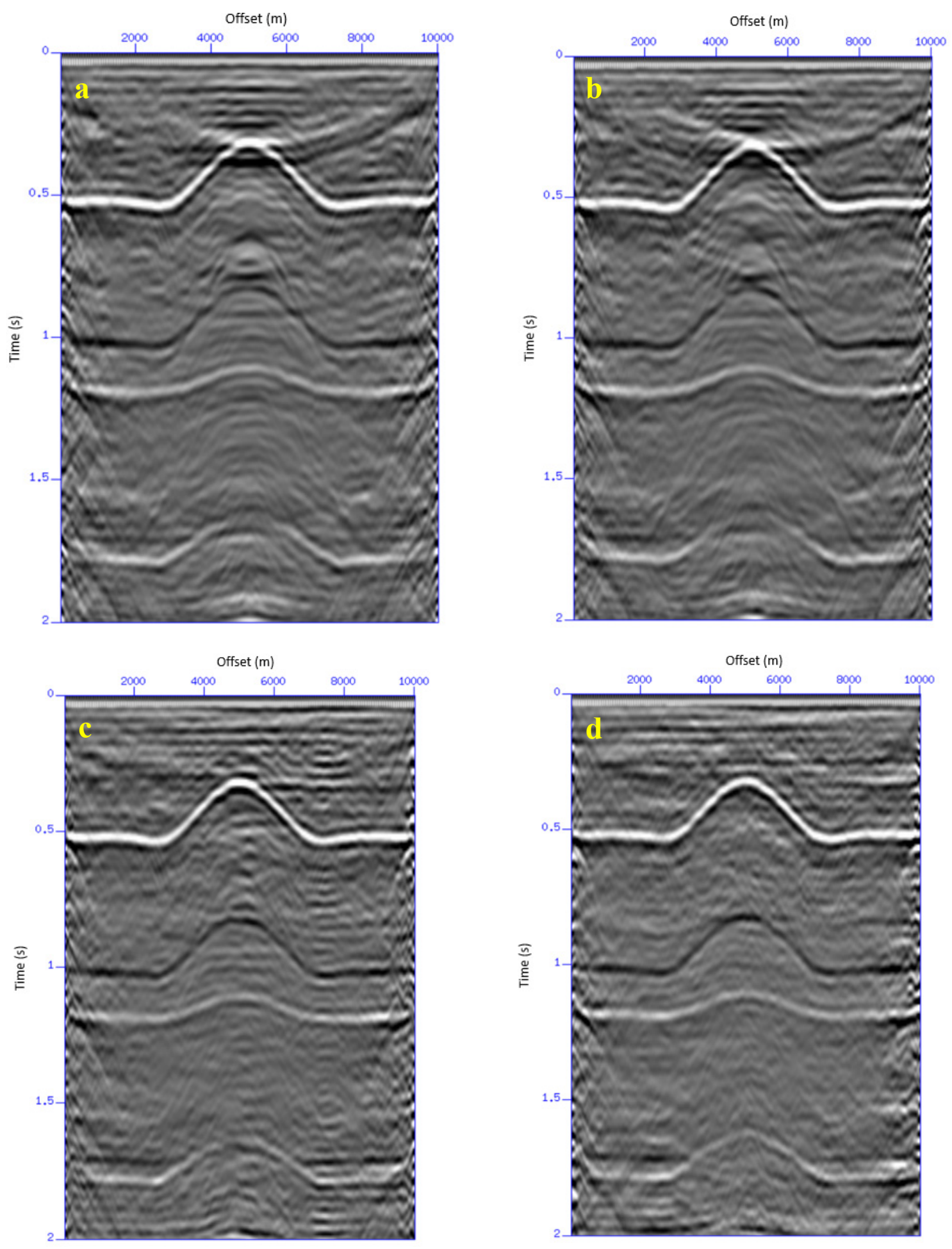

Figure 22. (a), (b), (c) and (d) shows the unmigrated zero-offset sections obtained from boundaries 1, 2, 3, and 4 for 5 sources about the anticline model, respectively. 

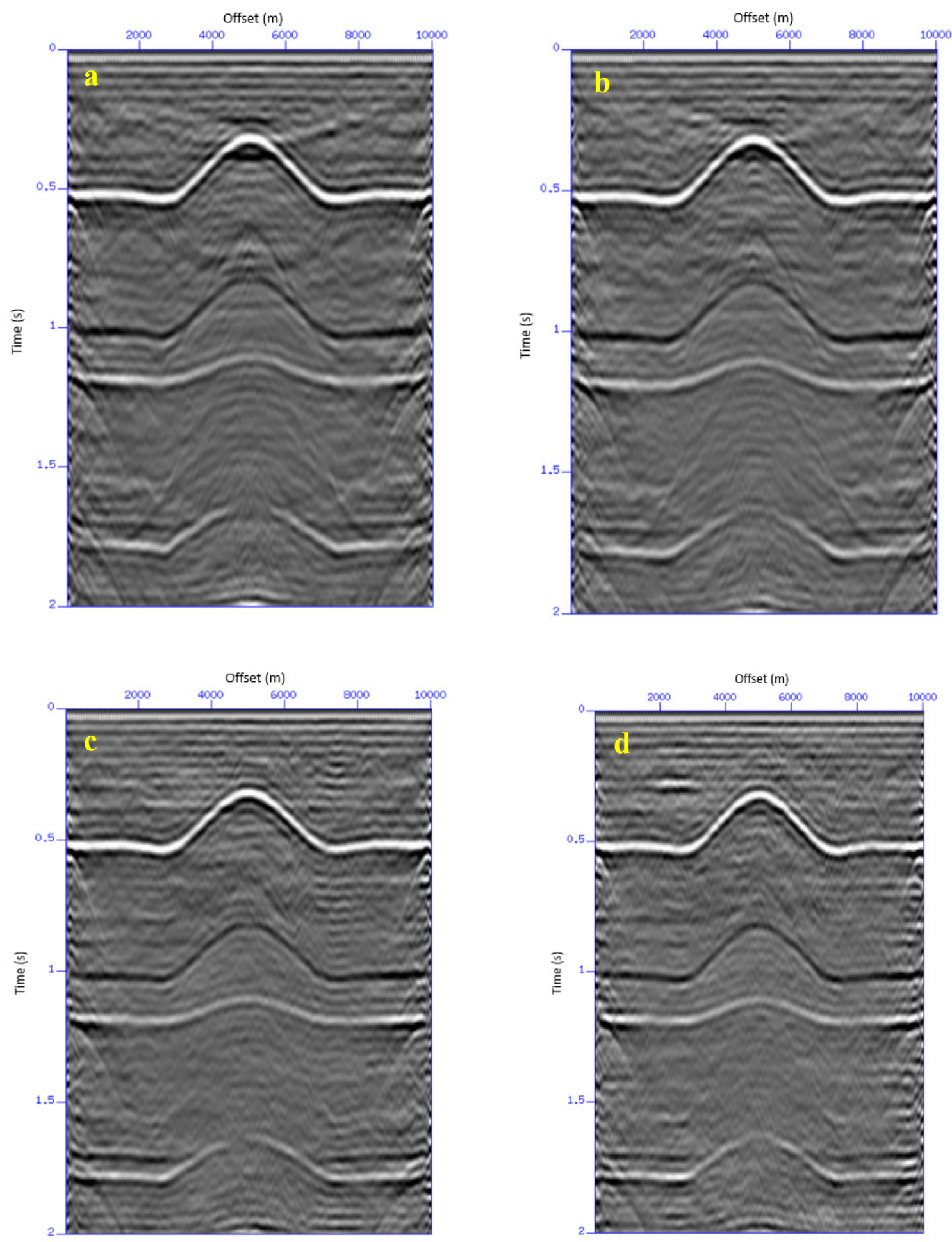

Figure 23. (a), (b), (c) and (d) shows the unmigrated zero-offset sections obtained from boundaries 1, 2, 3, and 4 for 20 sources about the anticline model, respectively. 

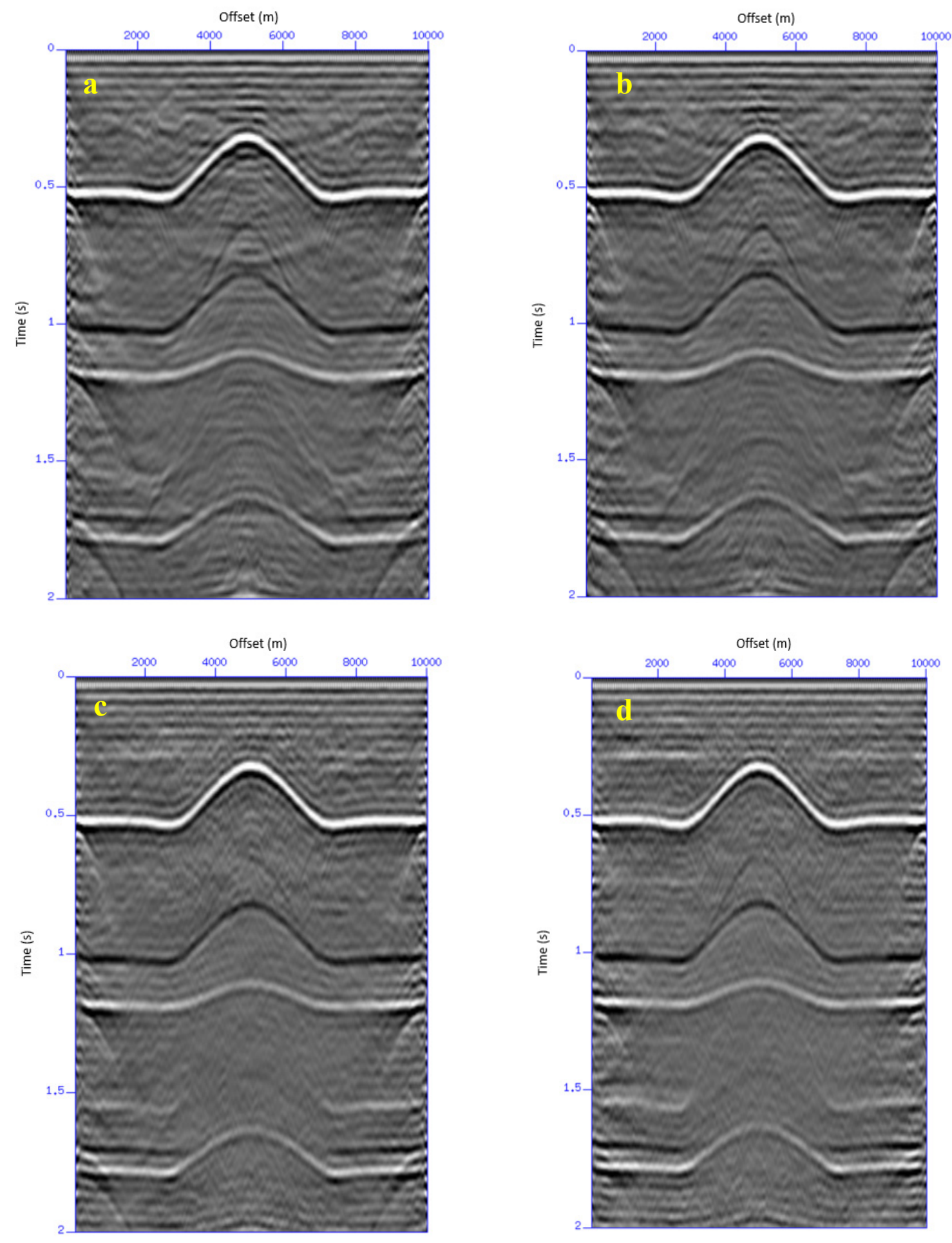

Figure 24. (a), (b), (c) and (d) shows the unmigrated zero-offset sections obtained from boundaries 1, 2, 3, and 4 for 500 sources about the anticline model, respectively. 

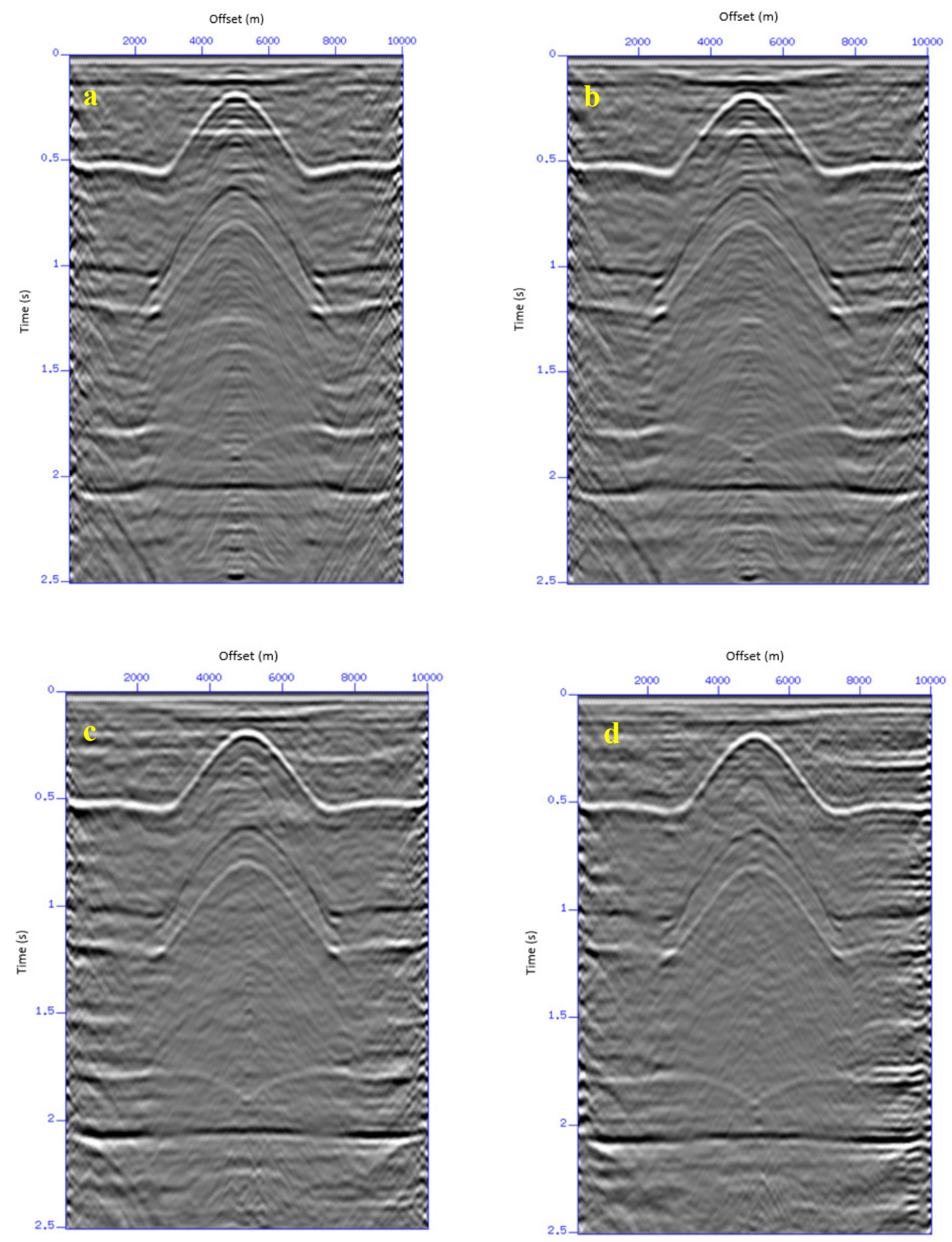

Figure 25. (a), (b), (c) and (d) shows the unmigrated zero-offset sections obtained from boundaries 1, 2, 3, and 4 for 5 sources about the anticline-syncline model, respectively. 

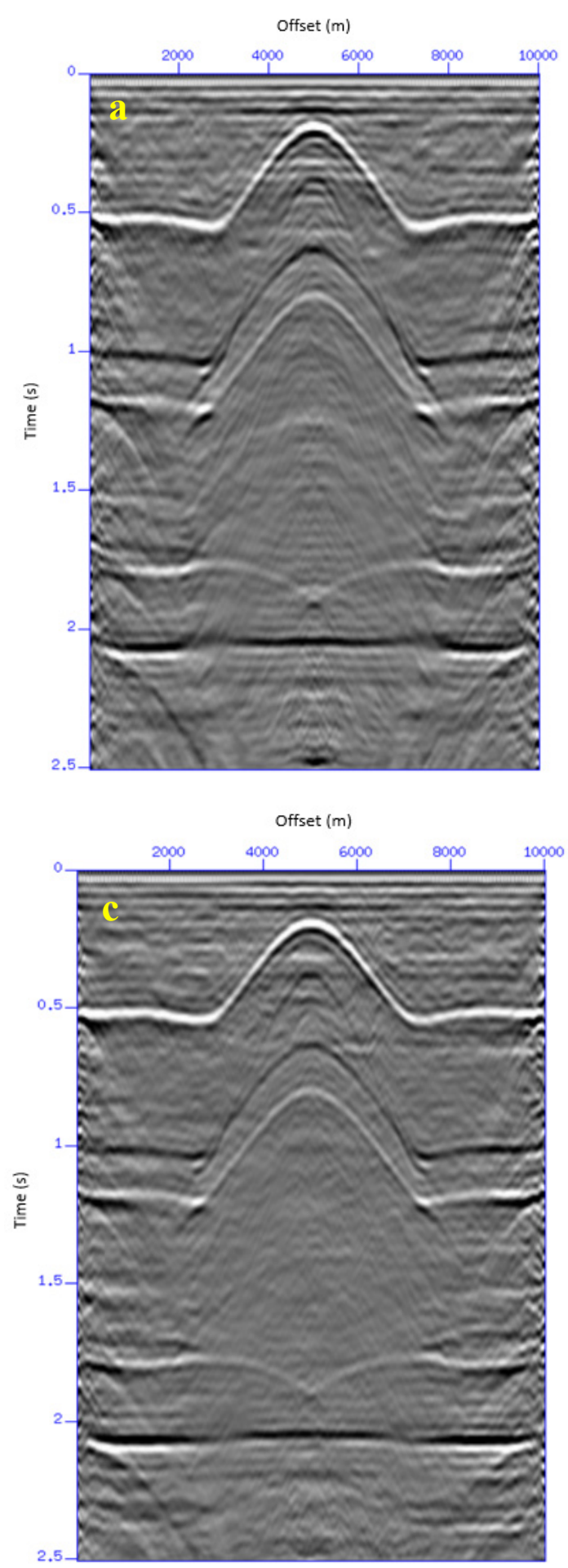
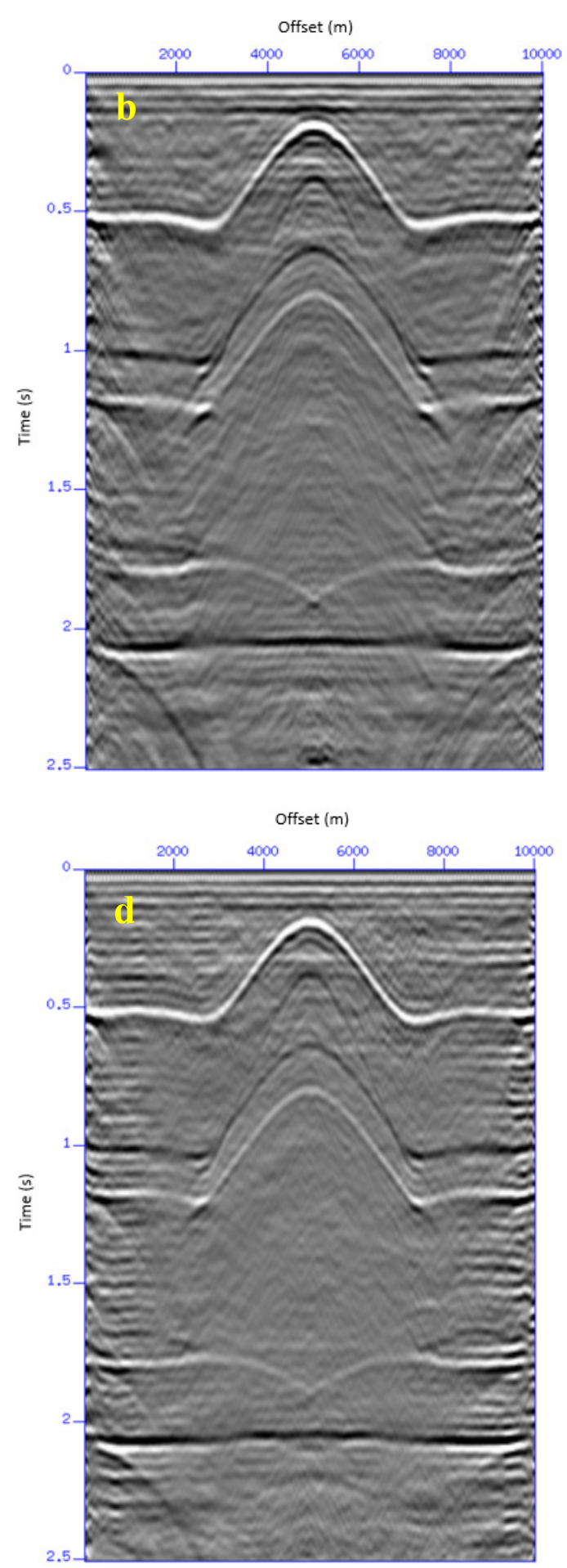

Figure 26. (a), (b), (c) and (d) shows the unmigrated zero-offset sections obtained from boundaries 1, 2, 3, and 4 for 20 sources about the anticline-syncline model, respectively. 

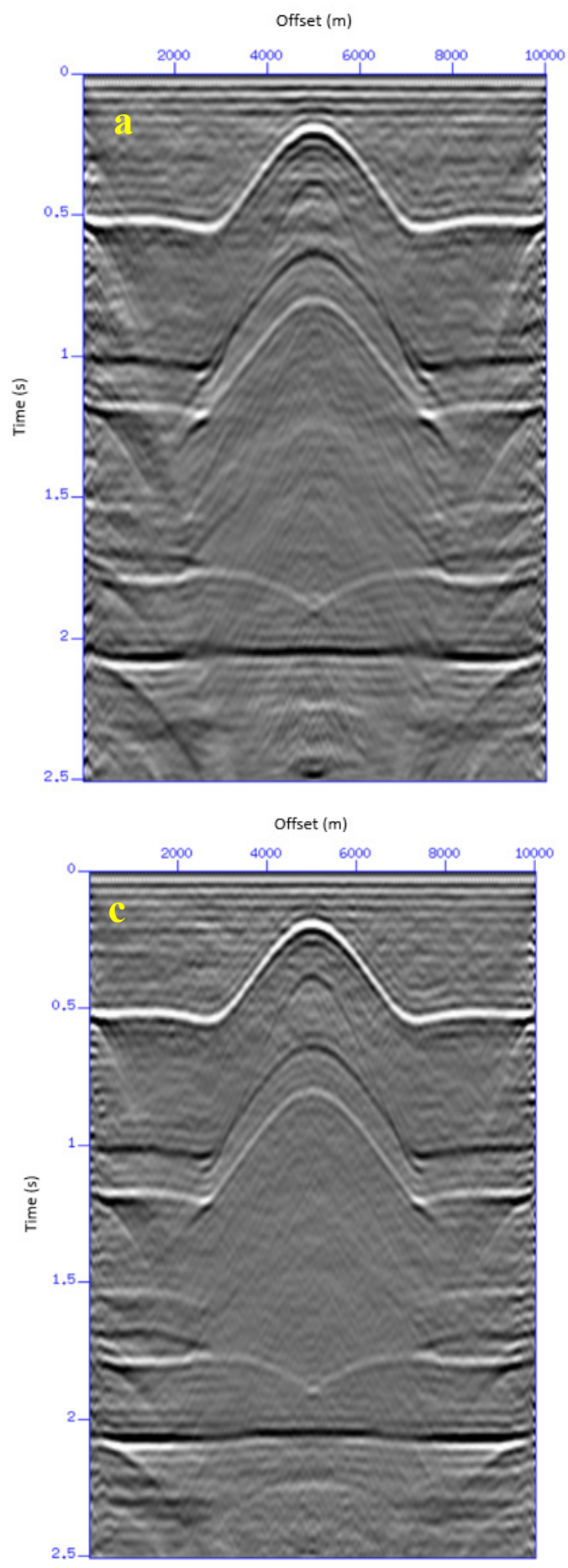
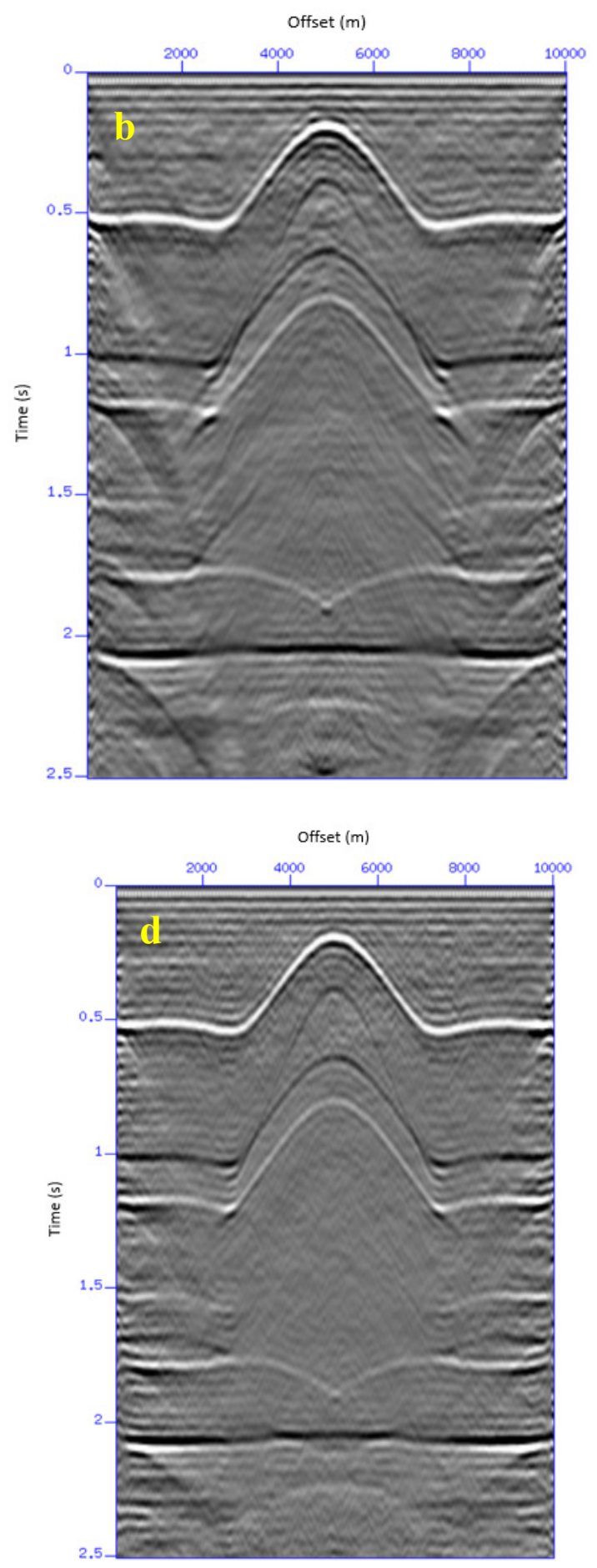

Figure 27. (a), (b), (c) and (d) shows the unmigrated zero-offset sections obtained from boundaries 1, 2, 3, and 4 for 500 sources about the anticline-syncline model, respectively. 
When examining the retrieved unmigrated stacked sections for the flat, the anticline and the anticline-syncline models, it is observed that when the number of sources increase, which means that underground is illuminated more angles, the $\mathrm{S} / \mathrm{N}$ ratio increases, and some artefacts become more improved as the noise boundary expands. These artefacts are clearly seen in the shallow parts of all models, especially when 5 sources are used.

\subsection{Zero-Offset VSP Geometry}

Figure 28 shows the noisy VSP data for the flat model using 20 randomly distributed sources in boundary 1 as an example. The horizontal axis shows the geophone number while the vertical axis shows the recording time. For the flat model, anticline model, and anticline-syncline model, I got 36 noisy VSP data for boundaries 1, 2, 3, and 4.

After running models and obtaining uncorrelated noisy VSP data, I used MATLAB software for VSP cross-correlation, and I got 36 virtual VSP shot gather for flat, anticline, and anticline-syncline models regarding all boundaries.

Figures 29-30-31 show virtual VSP shot gathers for a flat model corresponding to the four boundaries for 5, 20, and 500 sources, respectively. Figures 32-33-34 show virtual VSP shot gathers for an anticline model corresponding to the four boundaries for 5, 20, and 500 sources, respectively. Finally, Figures 35-36-37 show virtual VSP shot gathers for an anticline-syncline model corresponding to the four boundaries for 5, 20, and 500 sources, respectively. In all virtual VSP shot gathers, red arrow shows downgoing primary wave or first-break curve, yellow arrows show downgoing multiples, and blue arrows show upgoing waves. 


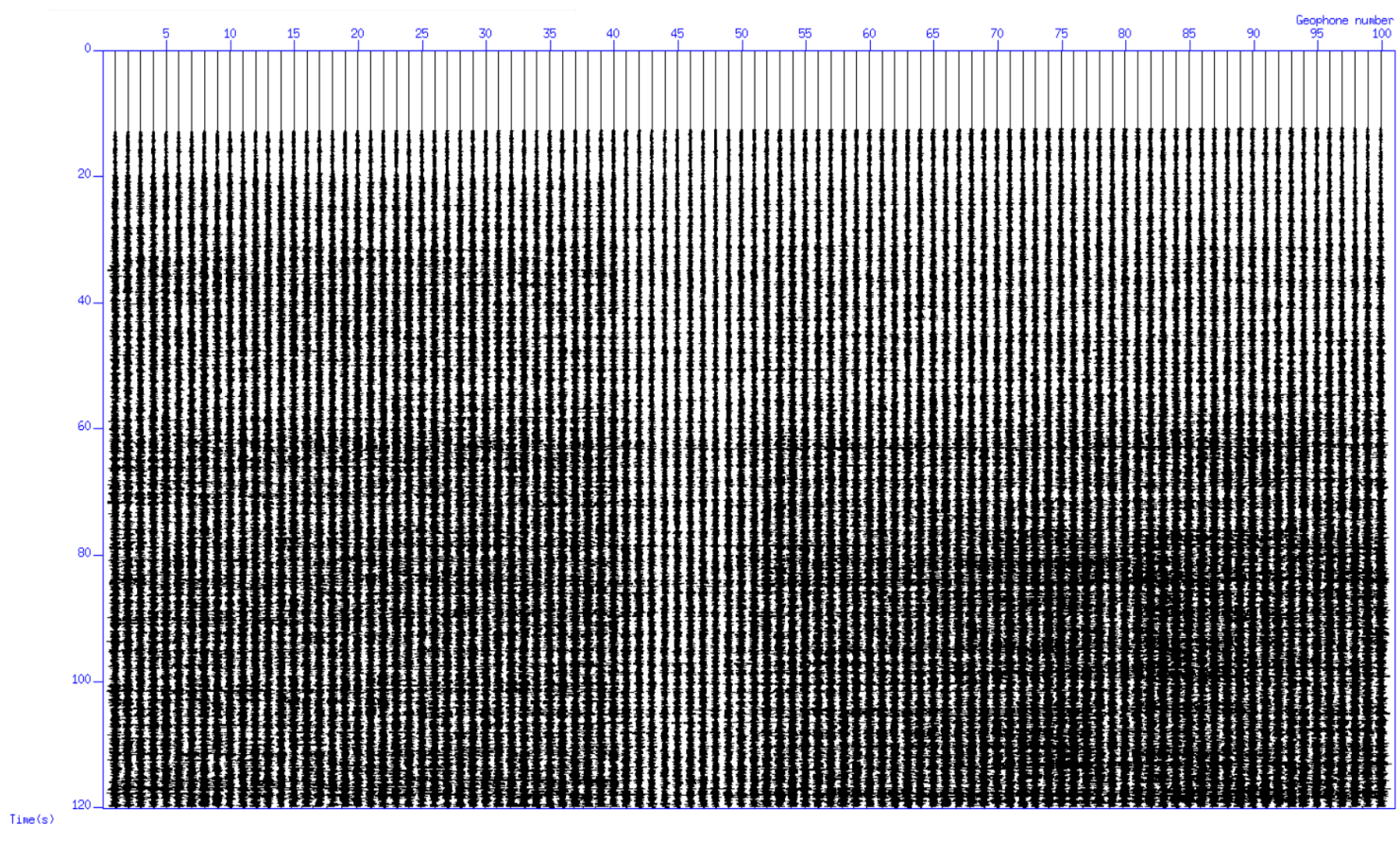

Figure 28. The noisy VSP data for the flat model using 20 randomly distributed sources in boundary 1 as an example. The horizontal axis shows the geophone number while the vertical axis shows the recording time. 
(a)

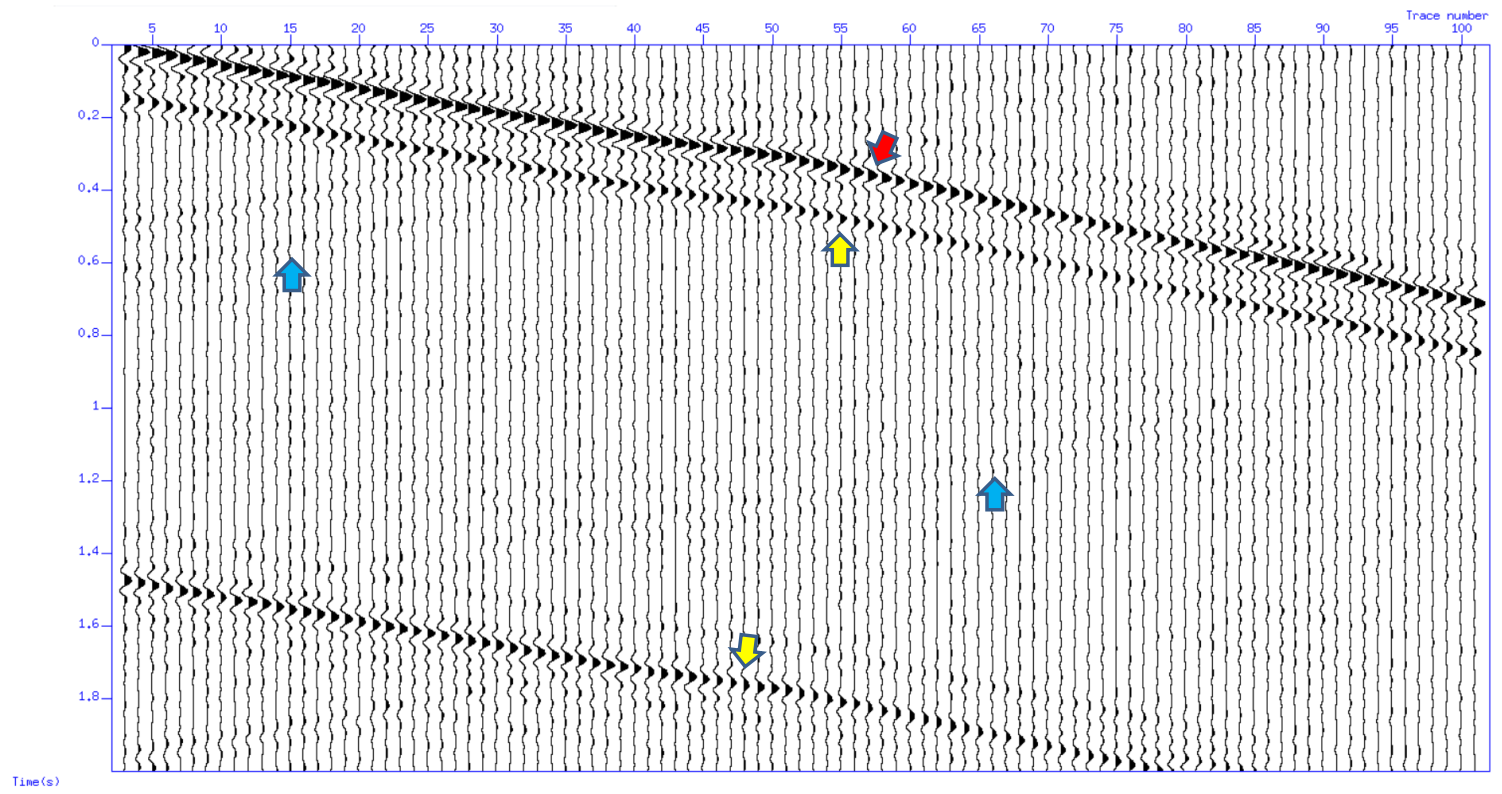

(b)

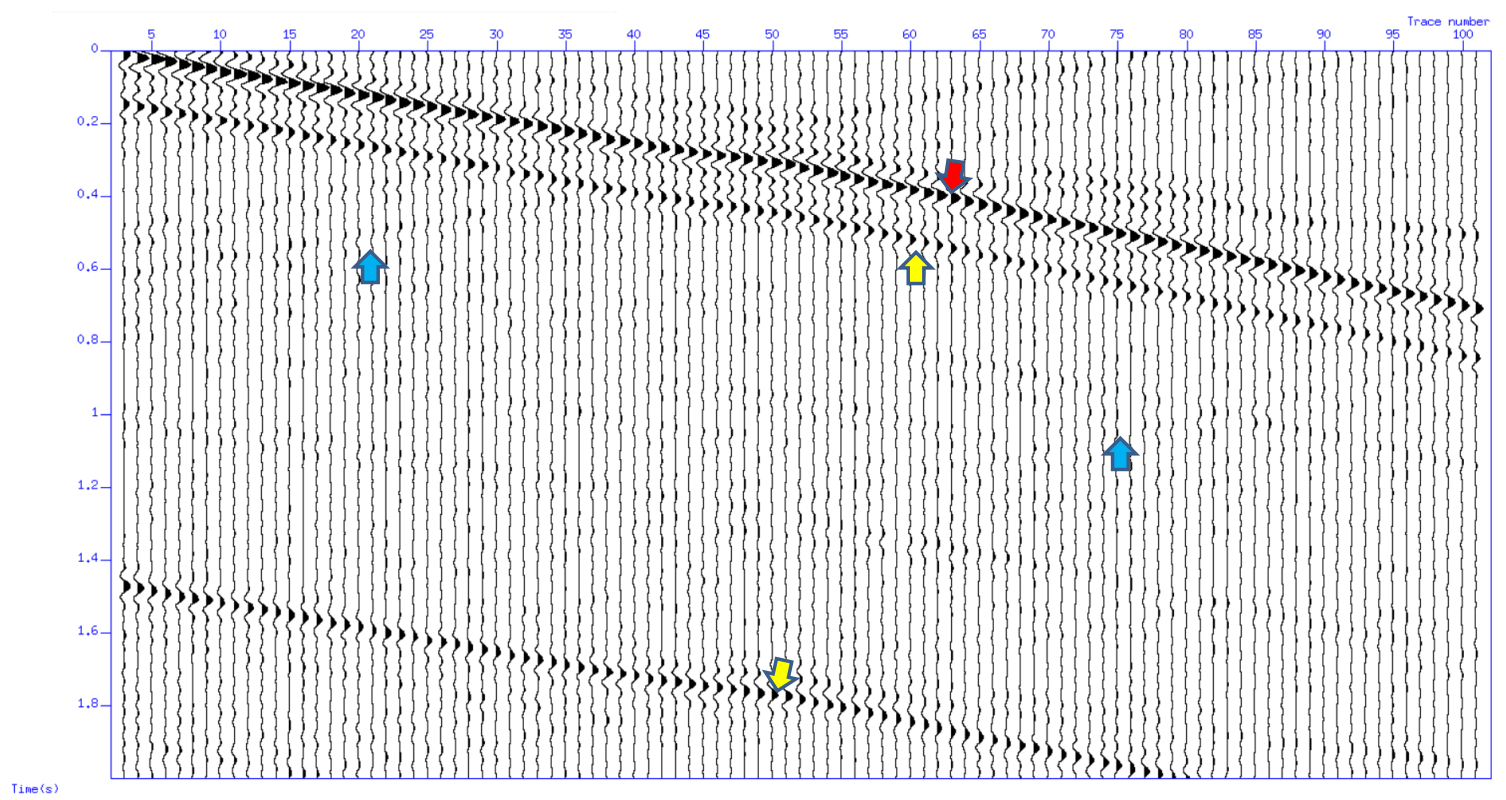


(c)

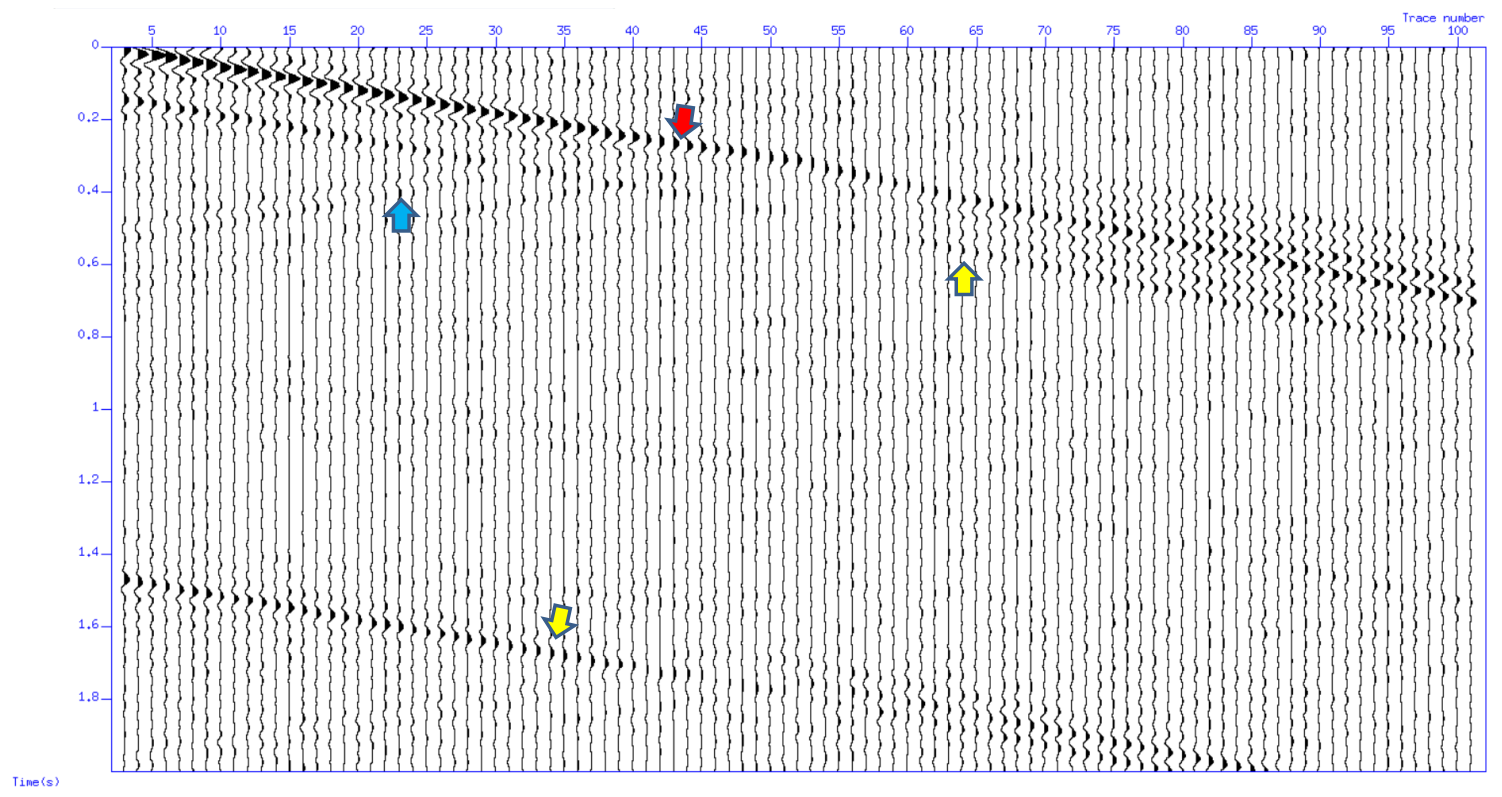

(d)

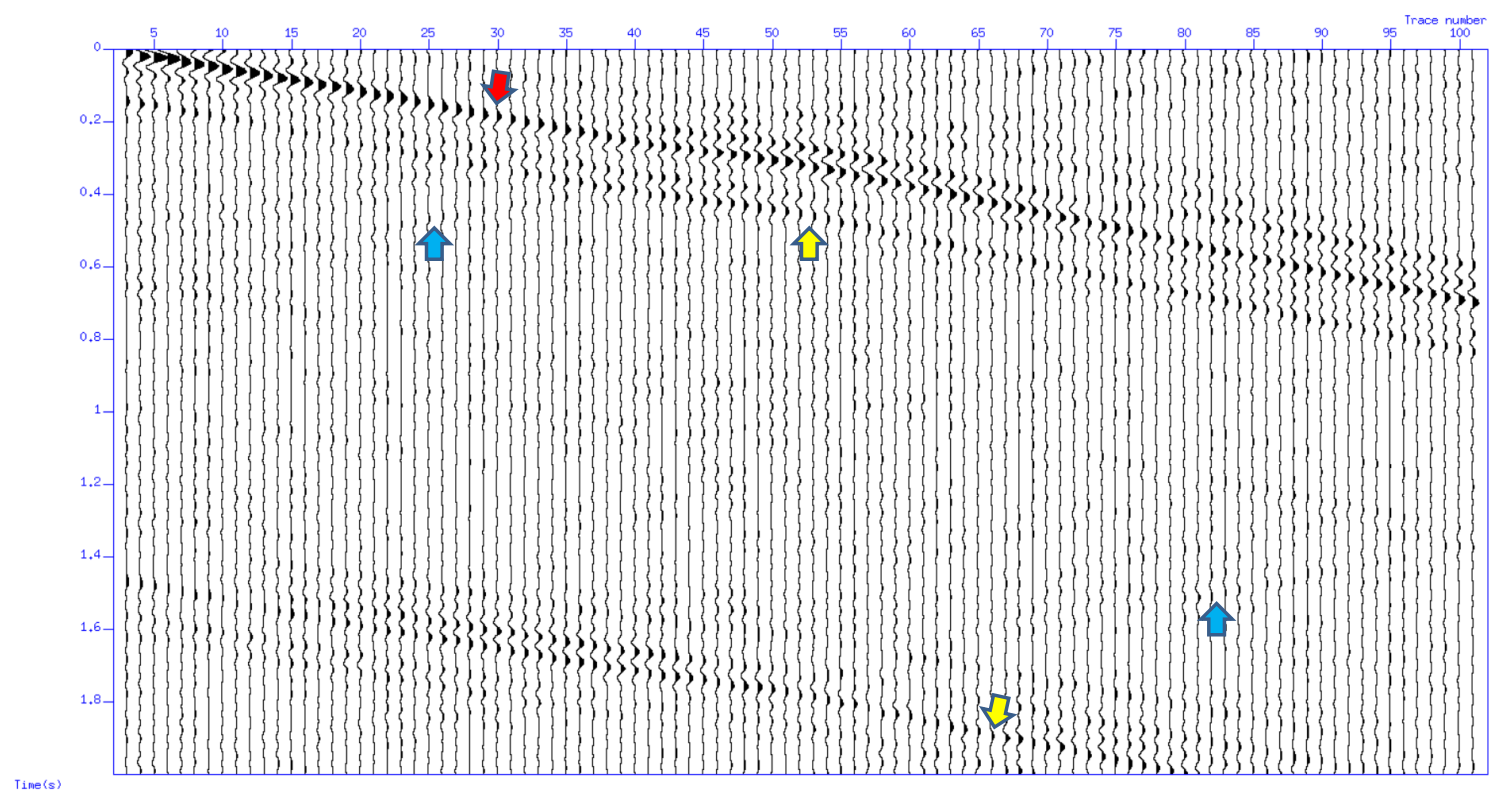

Figure 29. (a), (b), (c), and (d) show virtual VSP shot gather after cross-correlation for boundaries 1, 2, 3, and 4 for the flat model using 5 sources, respectively. The red arrow shows downgoing primary wave or first-break curve, the yellow arrows show downgoing multiples, and the blue arrows show upgoing waves. 
(a)

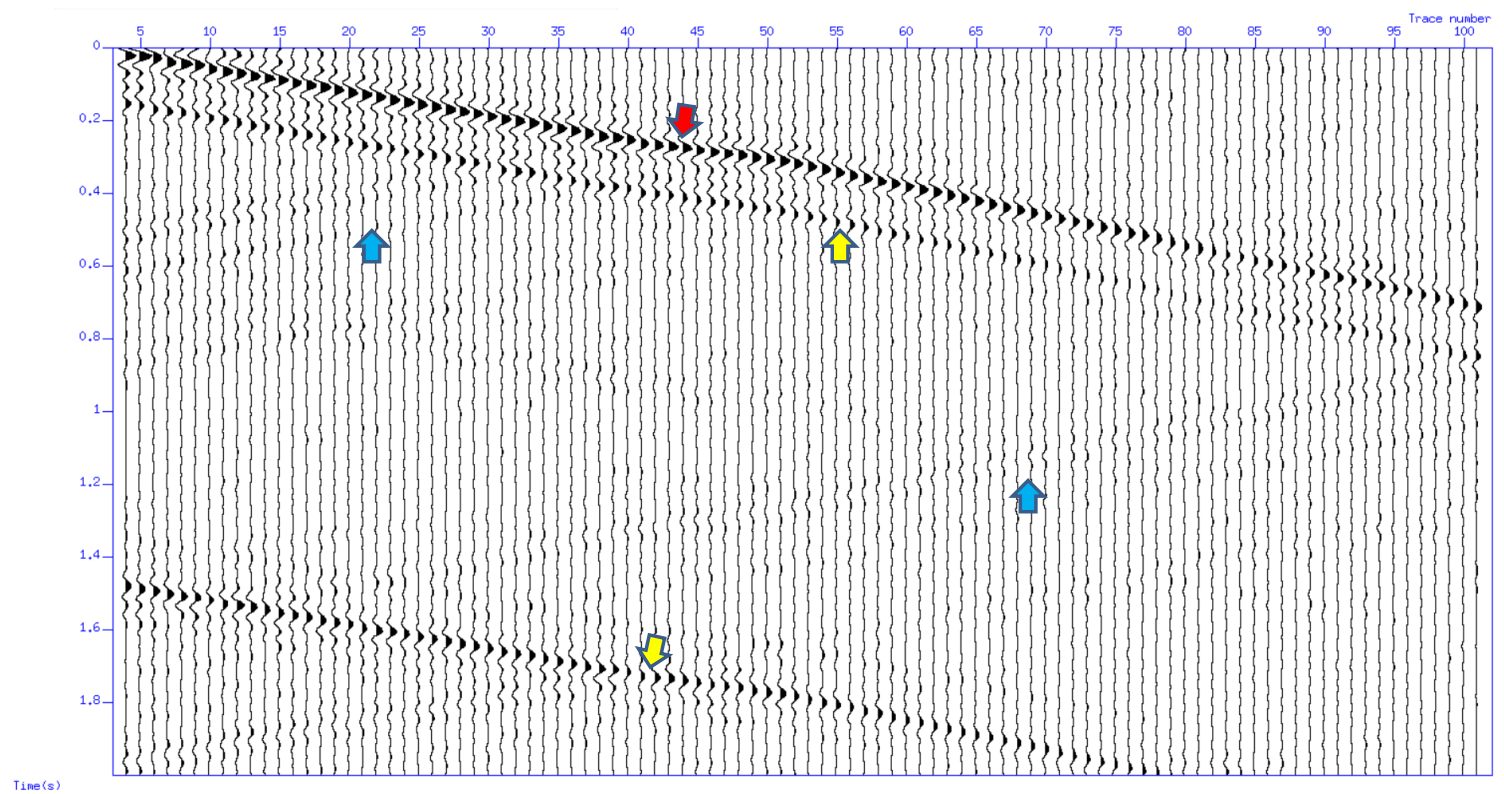

(b)

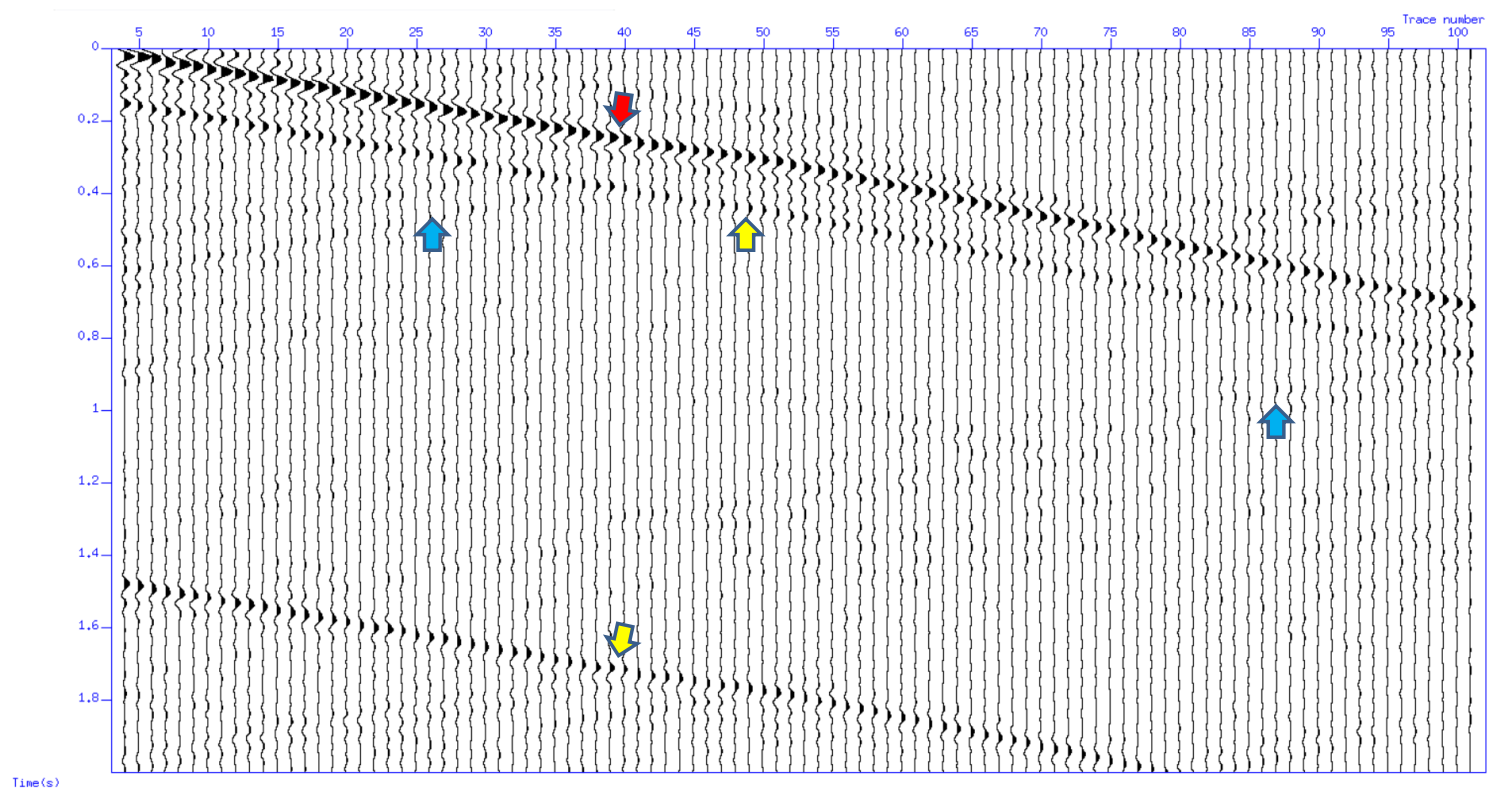


(c)

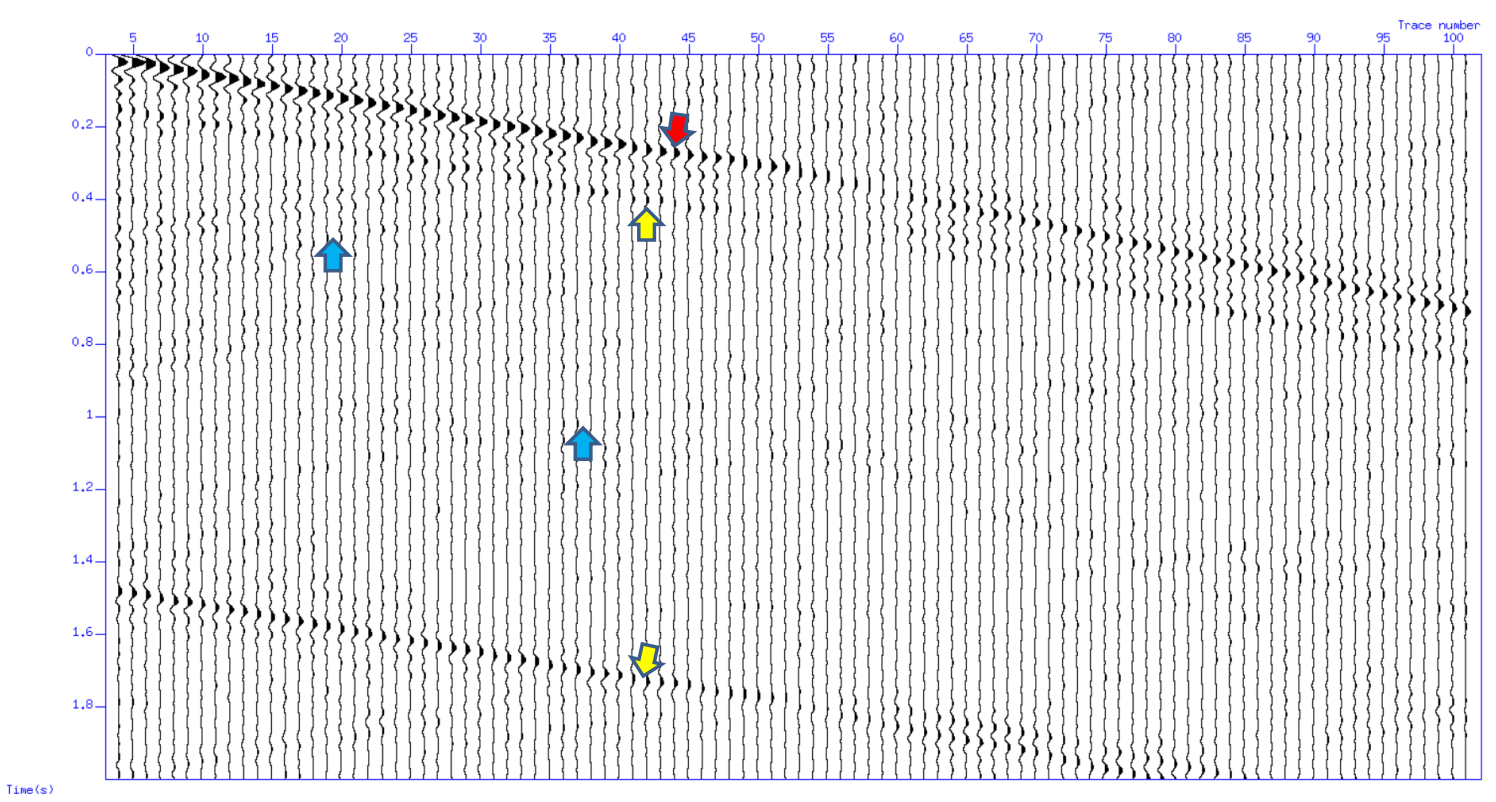

(d)

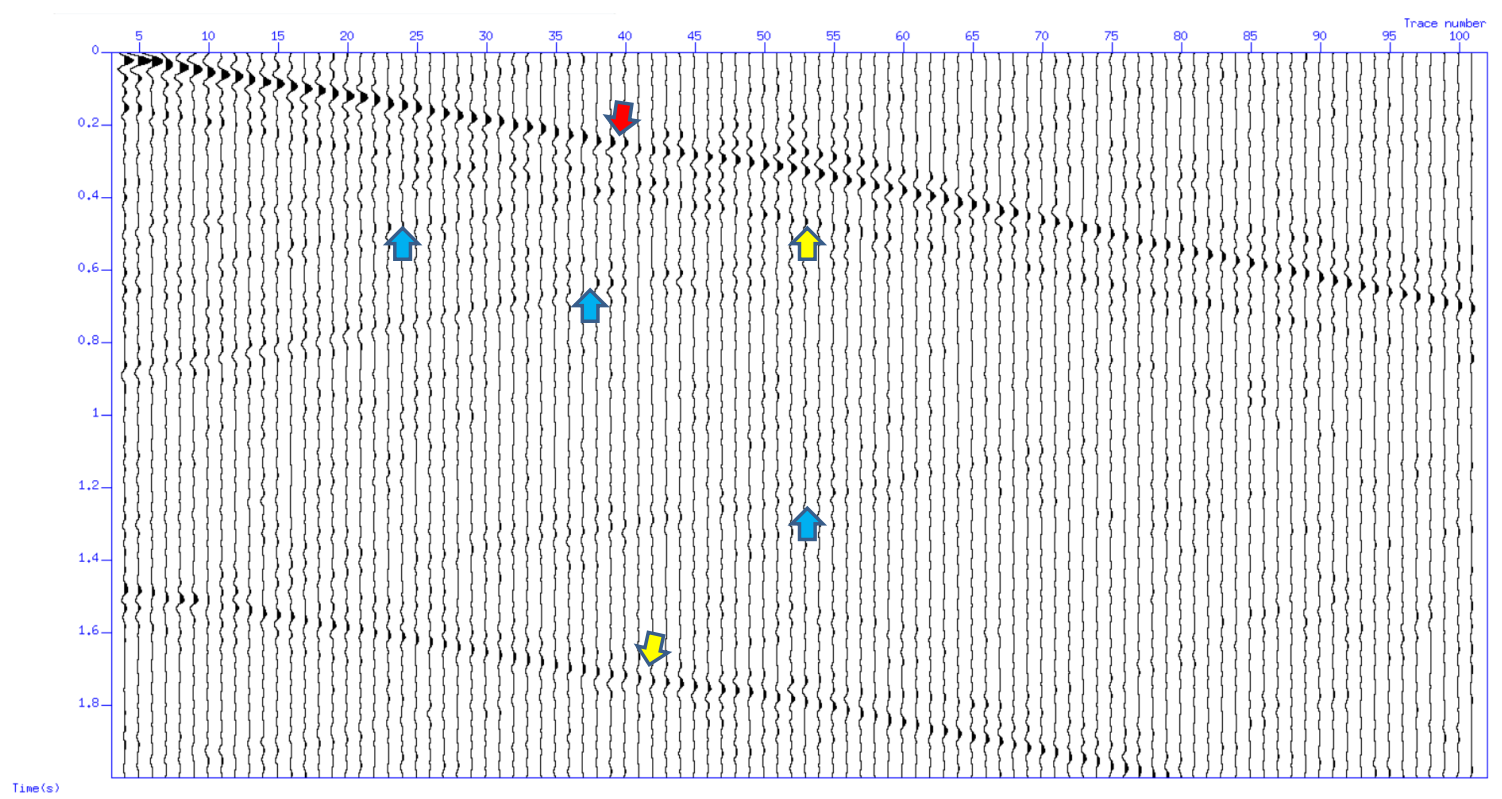

Figure 30. (a), (b), (c), and (d) show virtual VSP shot gather after cross-correlation for boundaries 1, 2, 3, and 4 for the flat model using 20 sources, respectively. The red arrow shows downgoing primary wave or first-break curve, the yellow arrows show downgoing multiples, and the blue arrows show upgoing waves. 
(a)

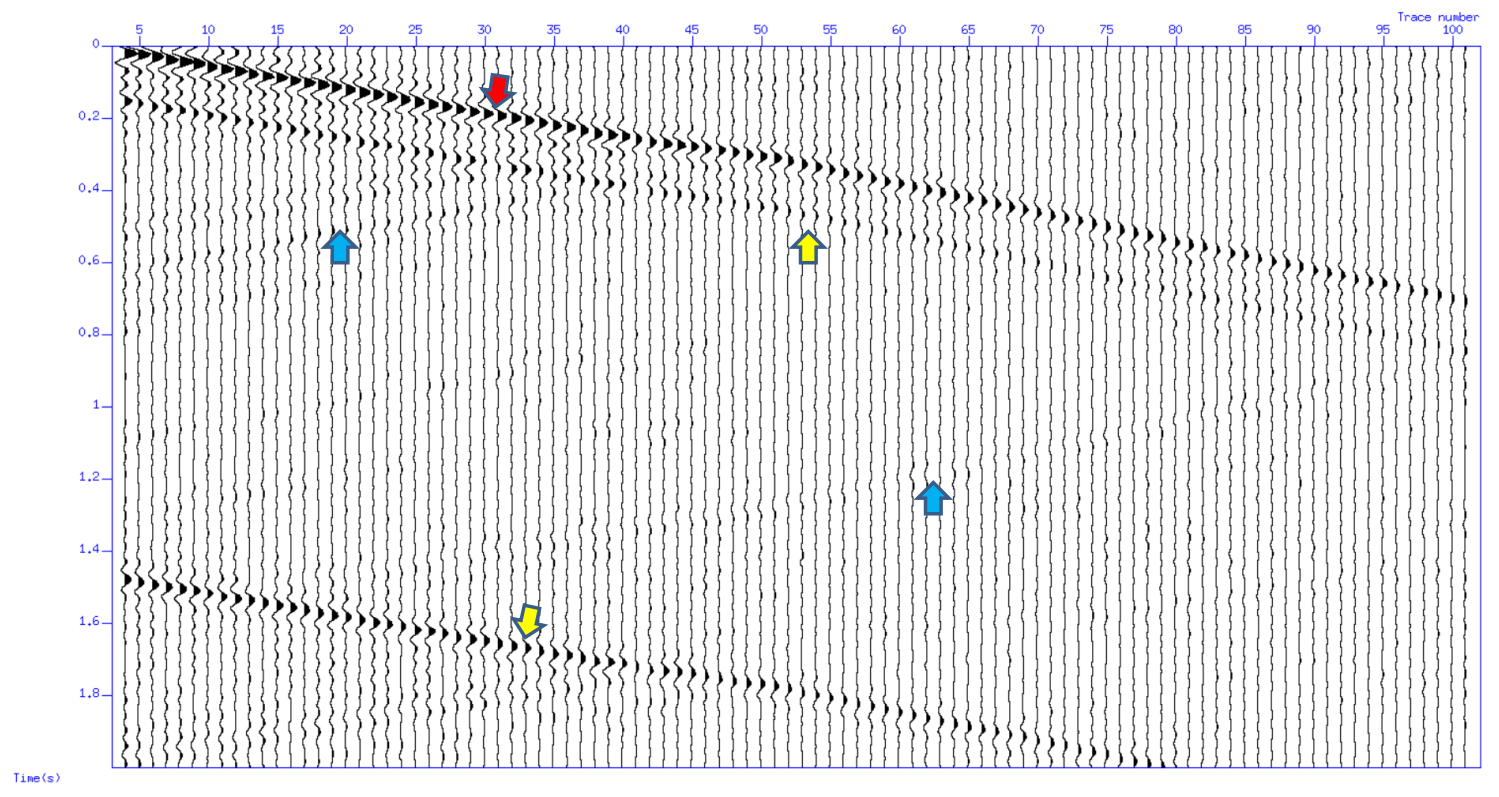

(b)

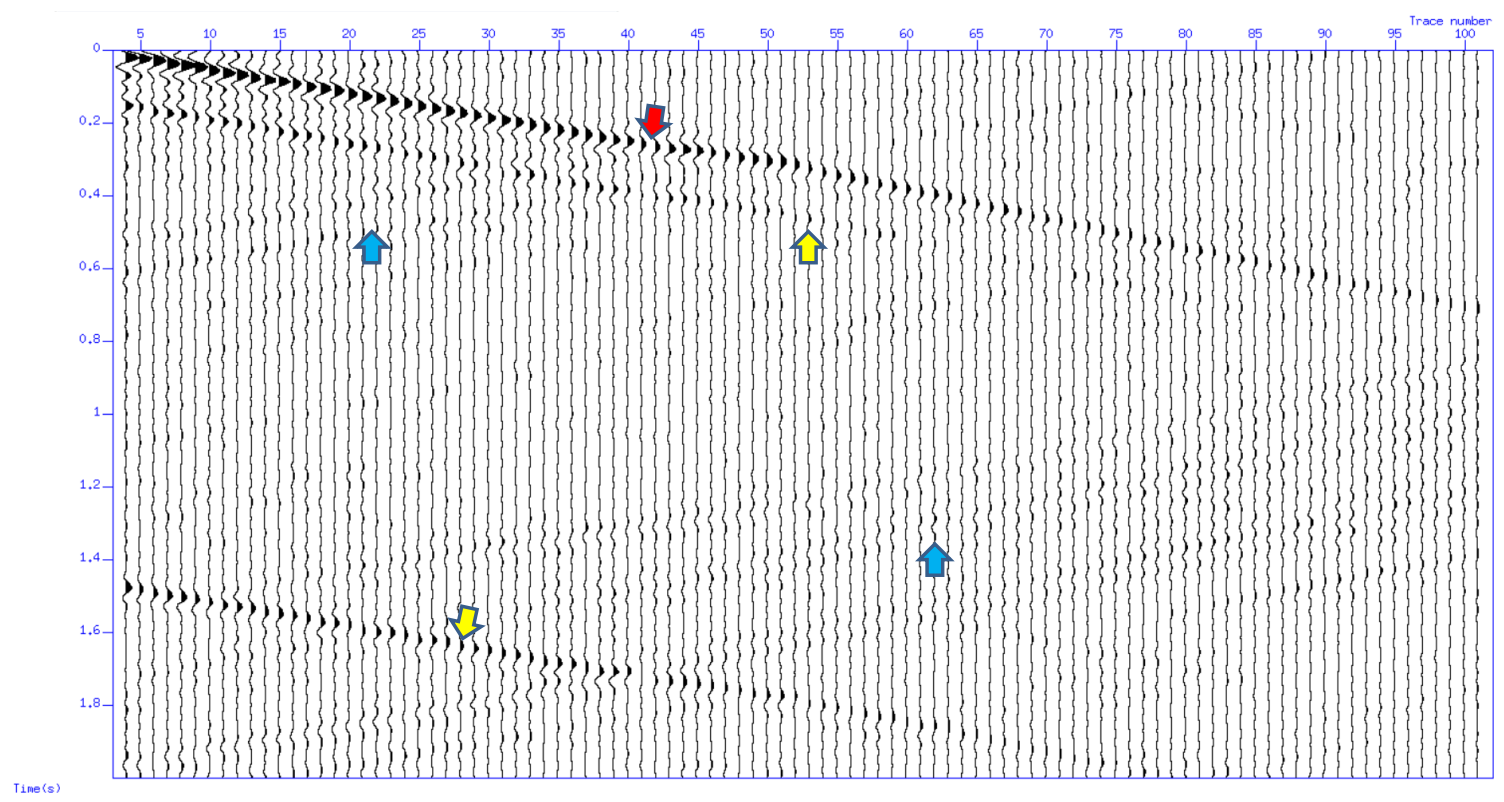


(c)

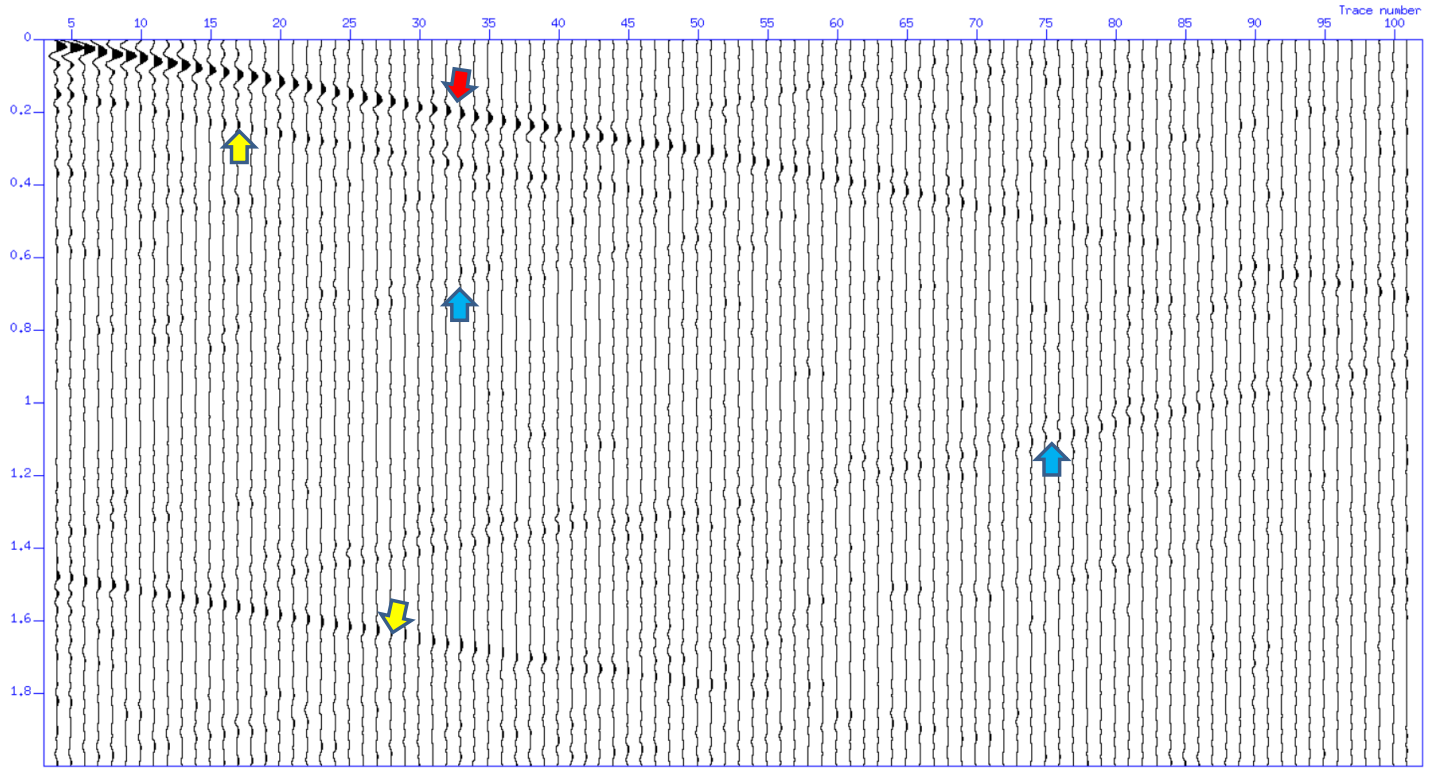

(d)

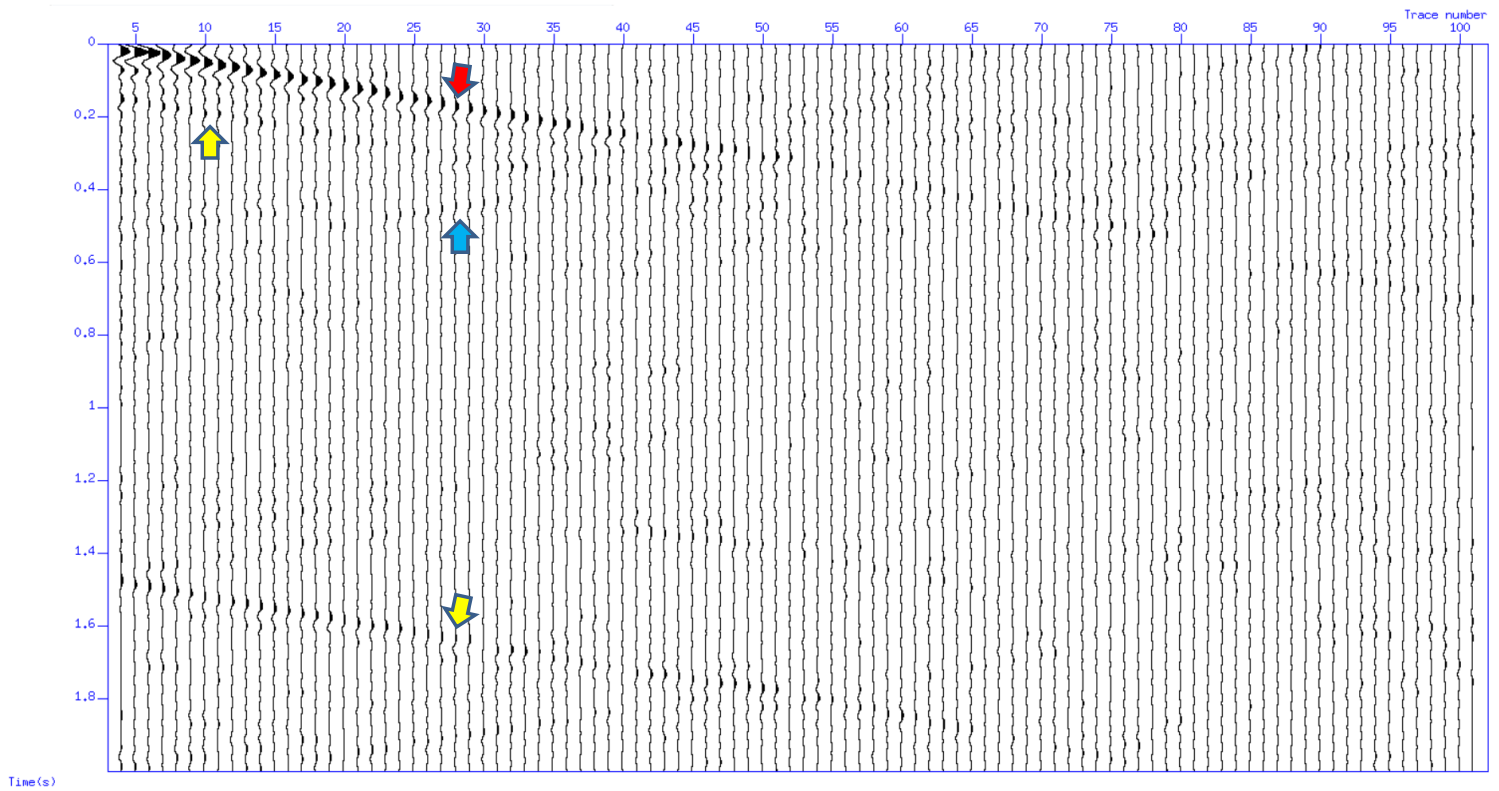

Figure 31. (a), (b), (c), and (d) show virtual VSP shot gather after cross-correlation for boundaries 1, 2, 3, and 4 for the flat model using 500 sources, respectively. The red arrow shows downgoing primary wave or first-break curve, the yellow arrows show downgoing multiples, and the blue arrows show upgoing waves. 
(a)

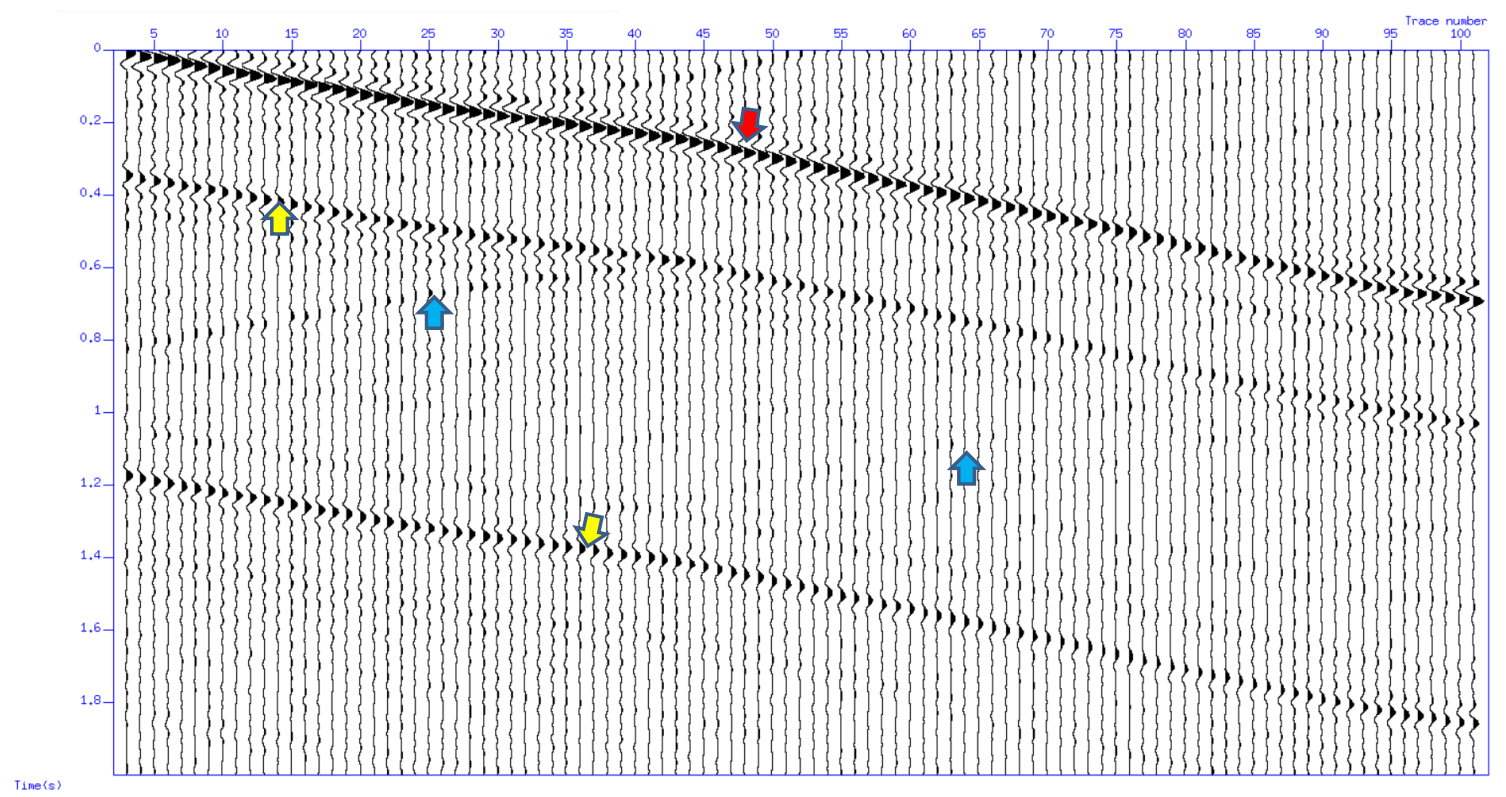

(b)

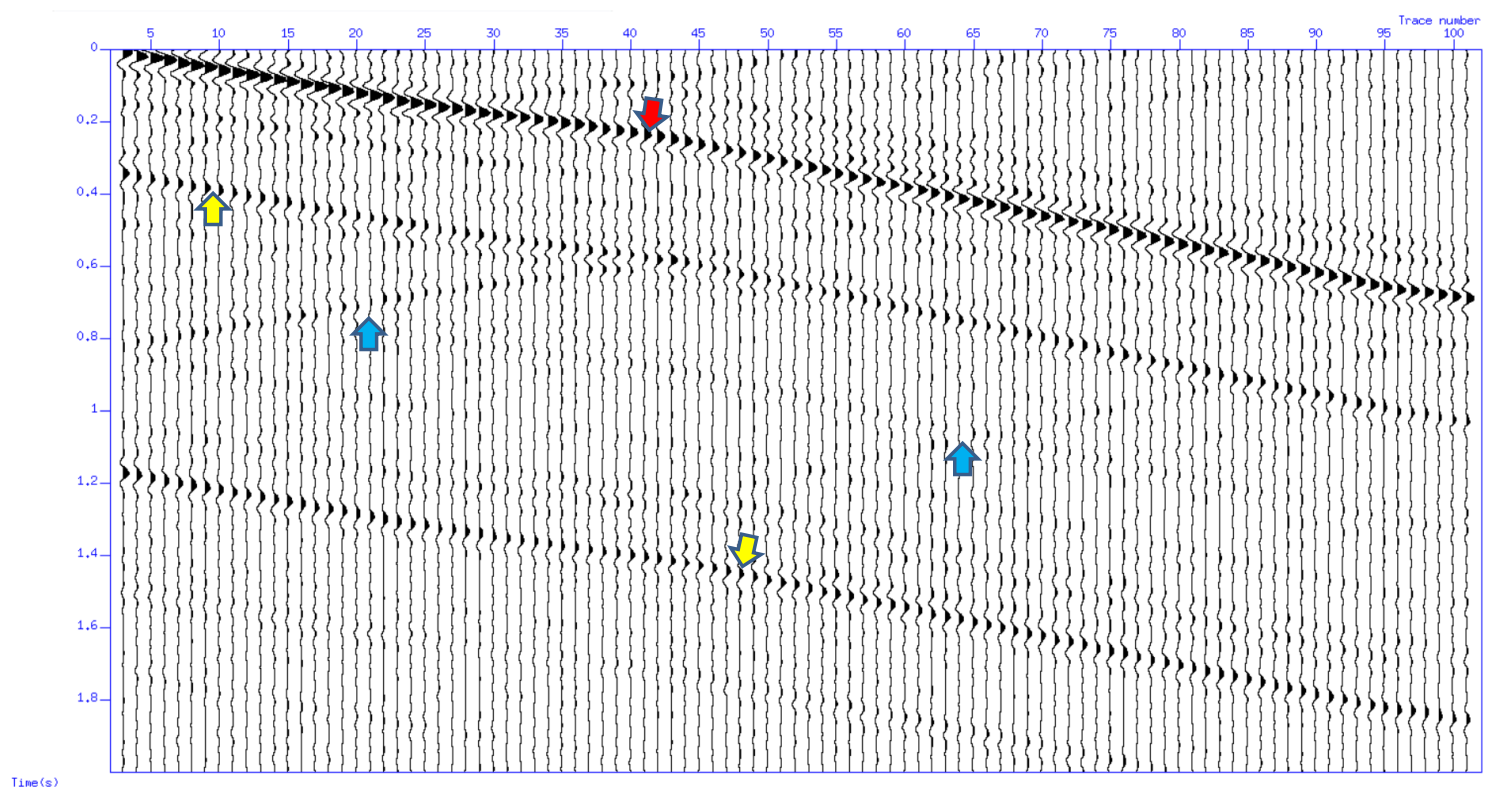


(c)

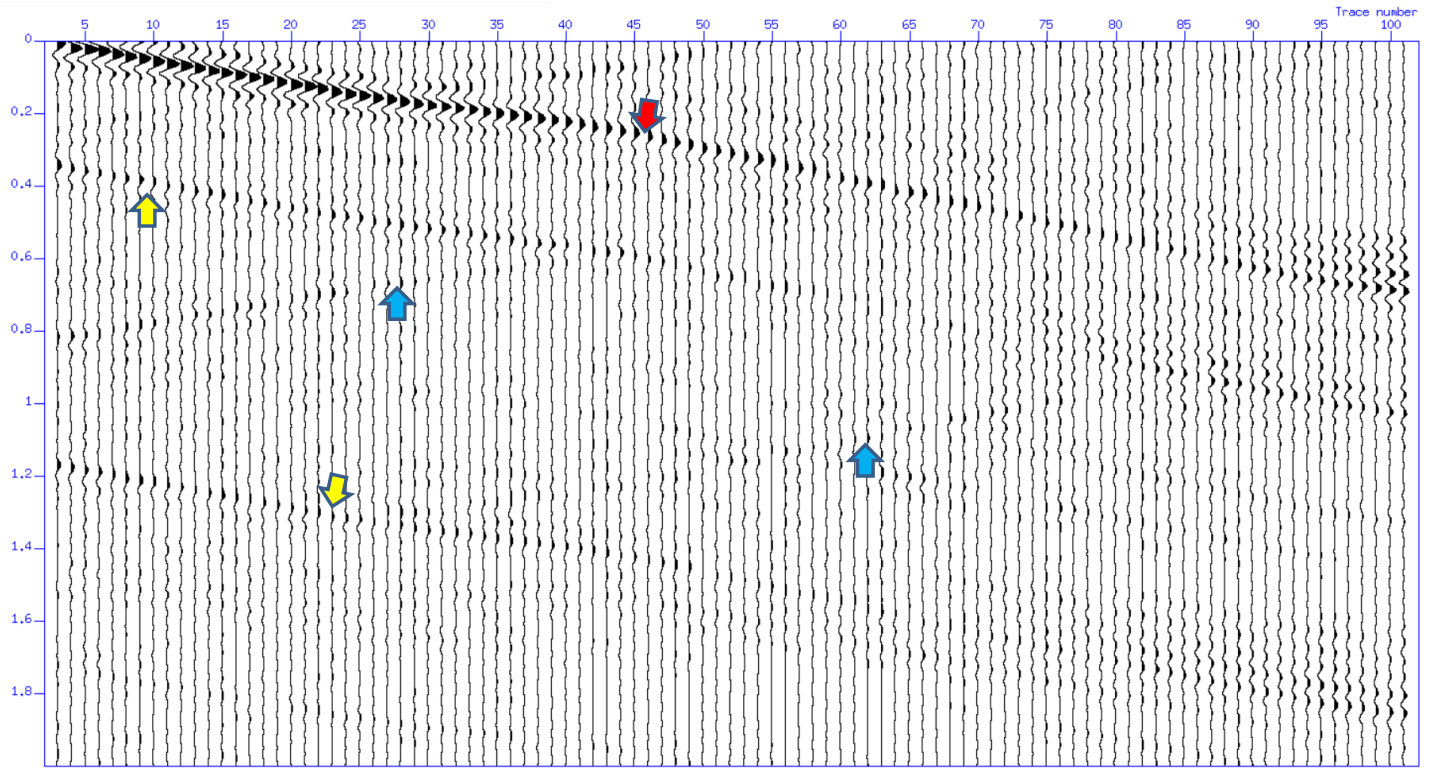

(d)

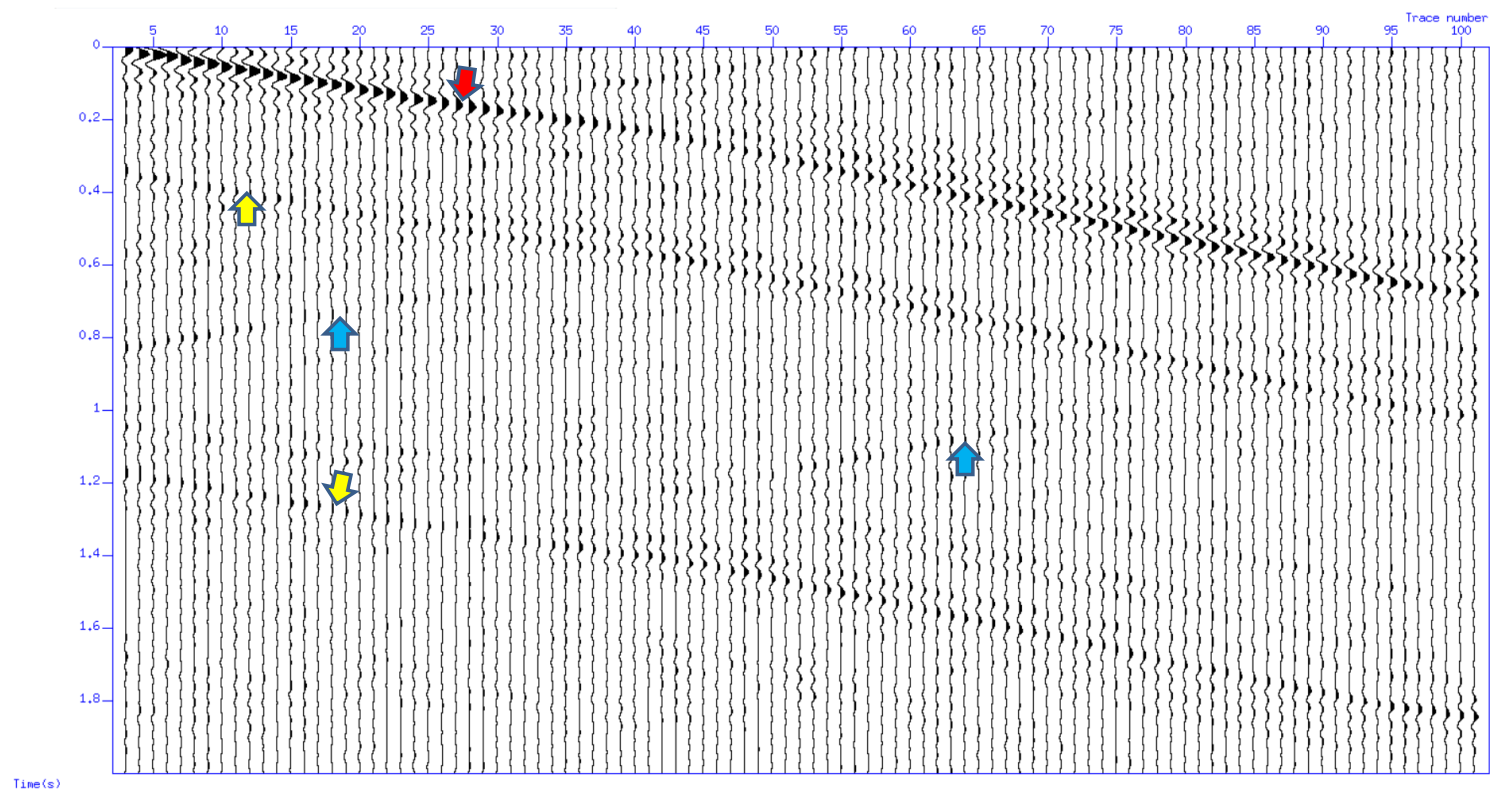

Figure 32. (a), (b), (c), and (d) show virtual VSP shot gather after cross-correlation for boundaries 1, 2, 3, and 4 for the anticline model using 5 sources, respectively. The red arrow shows downgoing primary wave or first-break curve, the yellow arrows show downgoing multiples, and the blue arrows show upgoing waves. 
(a)

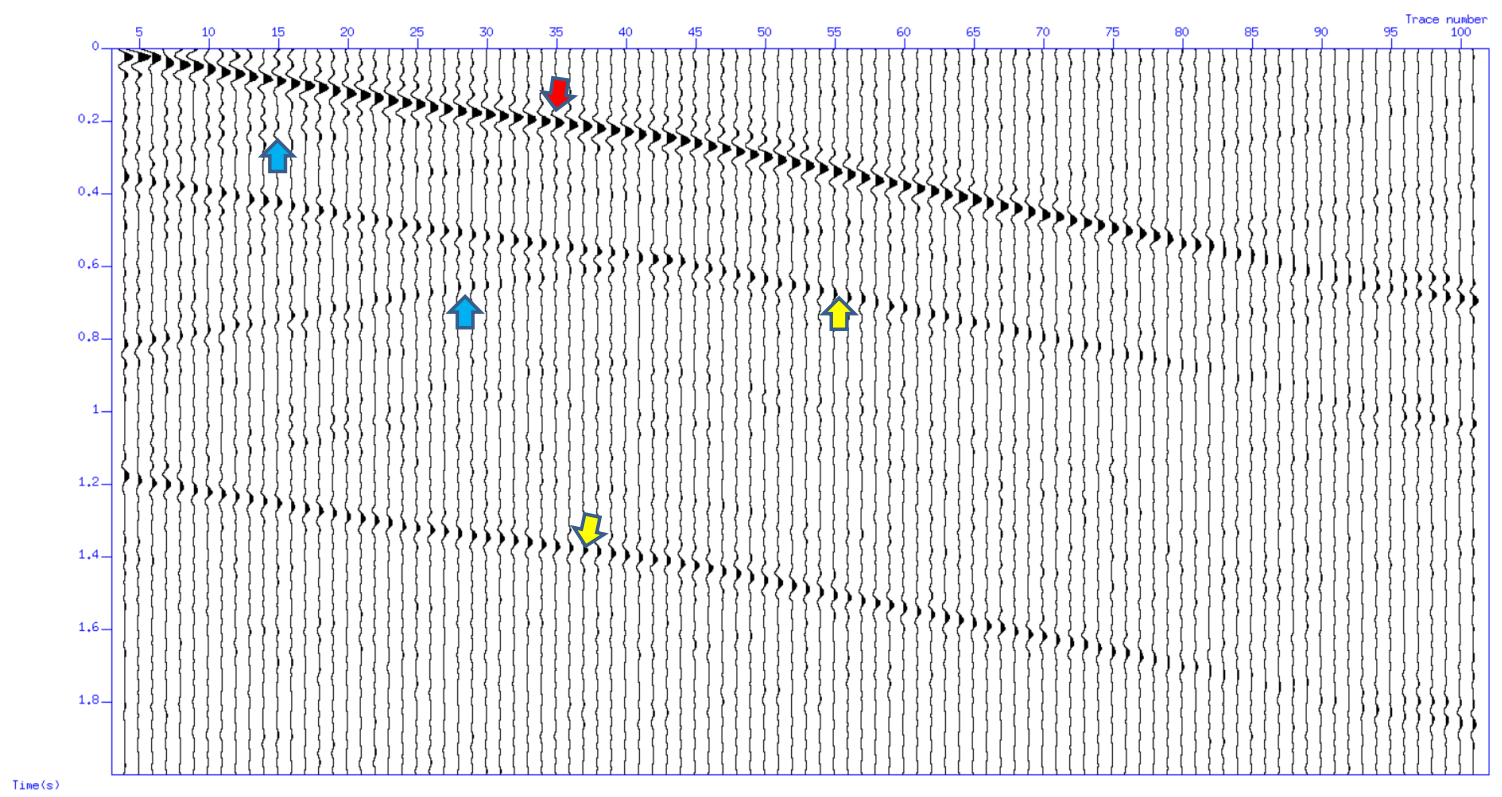

(b)

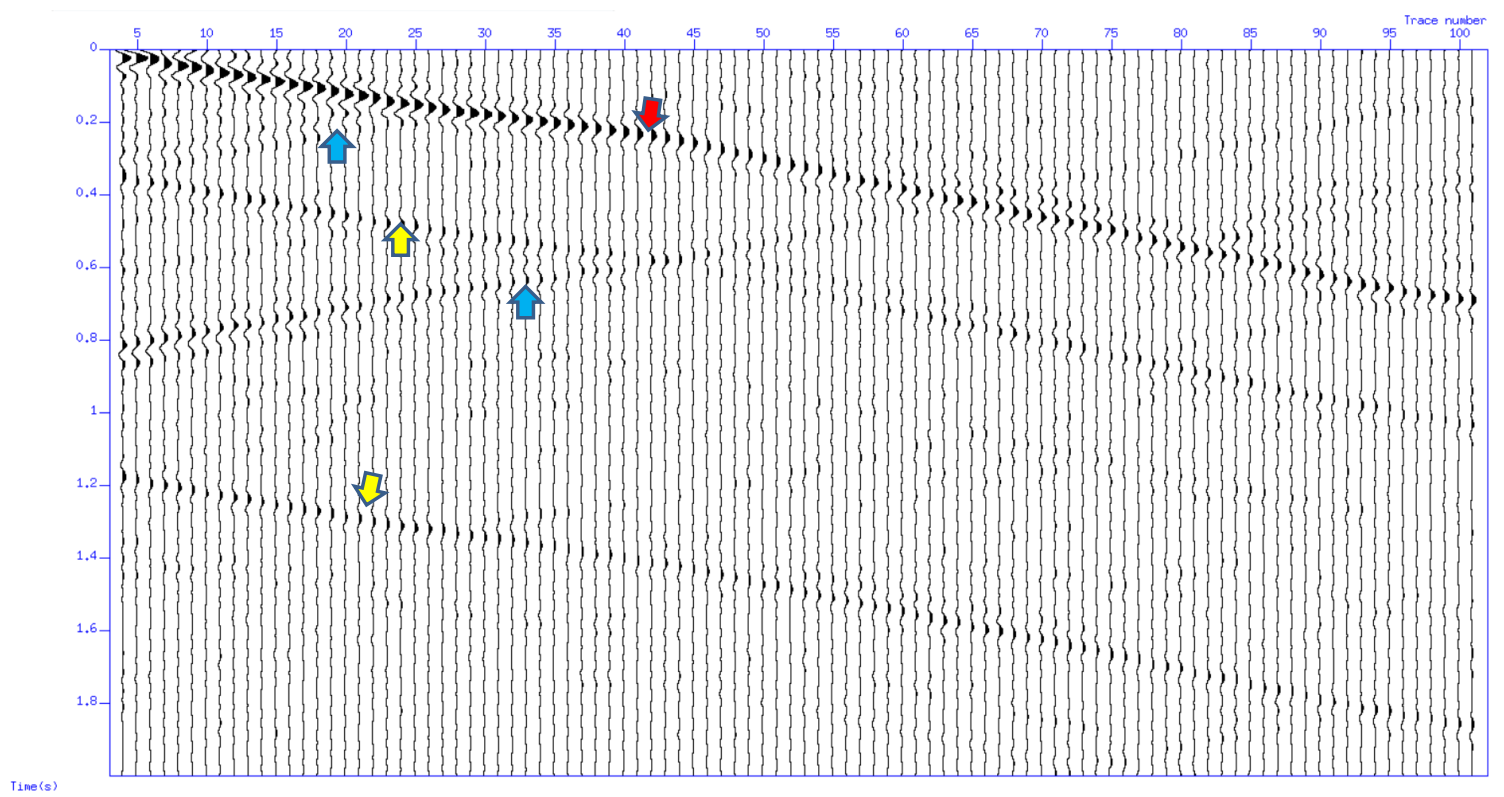


(c)

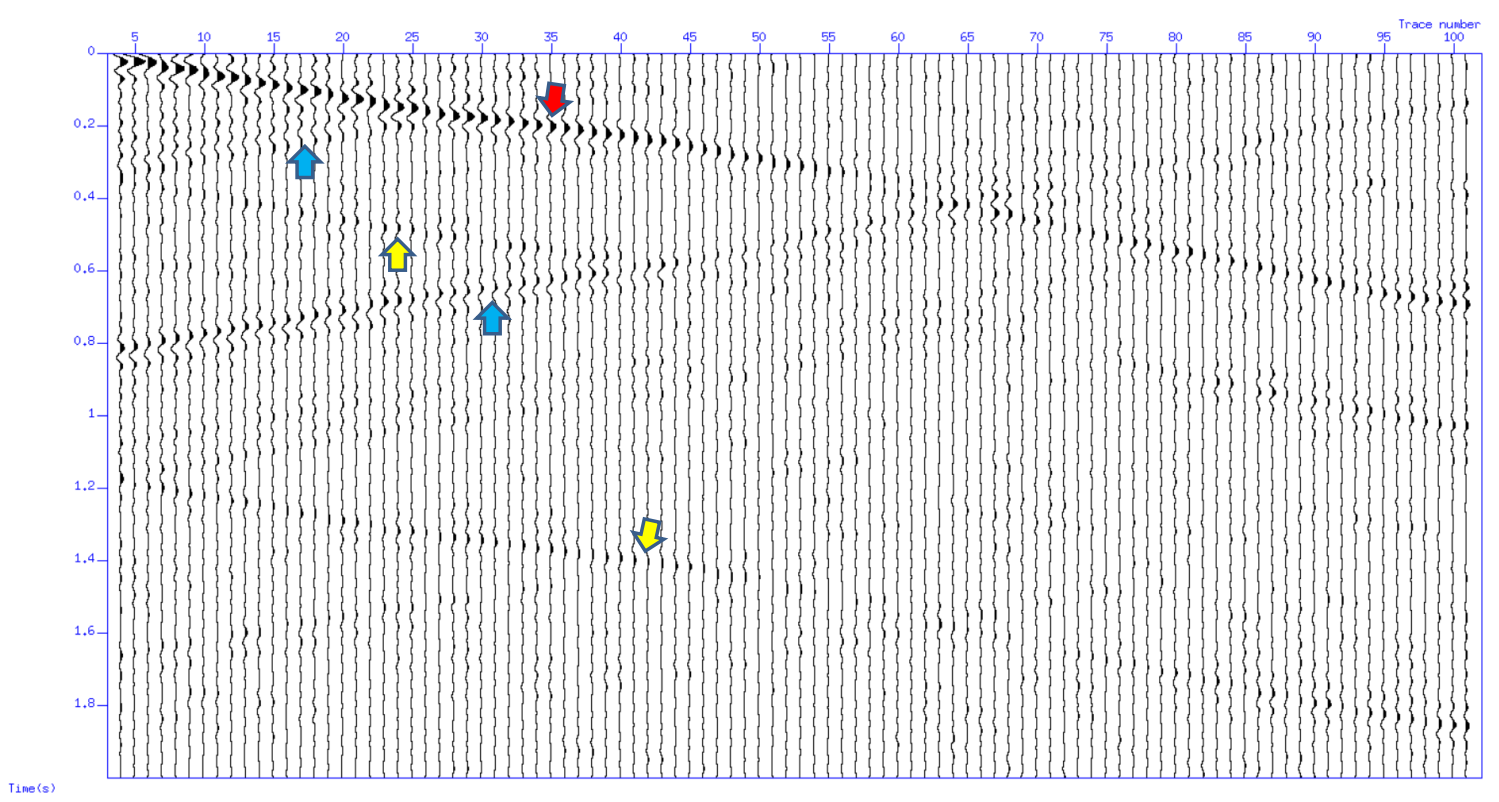

(d)

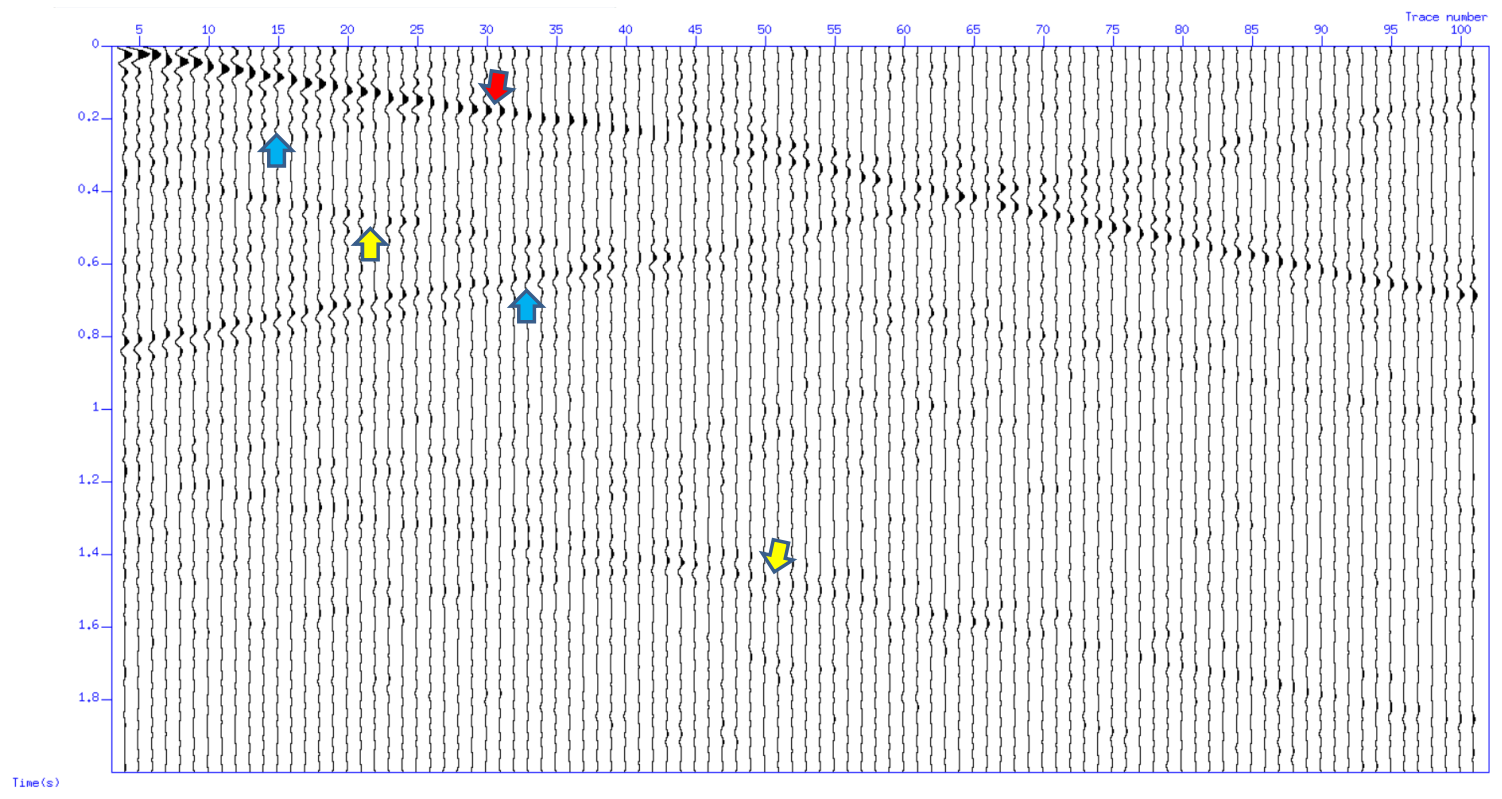

Figure 33. (a), (b), (c), and (d) show virtual VSP shot gather after cross-correlation for boundaries 1, 2, 3, and 4 for the anticline model using 20 sources, respectively. The red arrow shows downgoing primary wave or first-break curve, the yellow arrows show downgoing multiples, and the blue arrows show upgoing waves. 
(a)

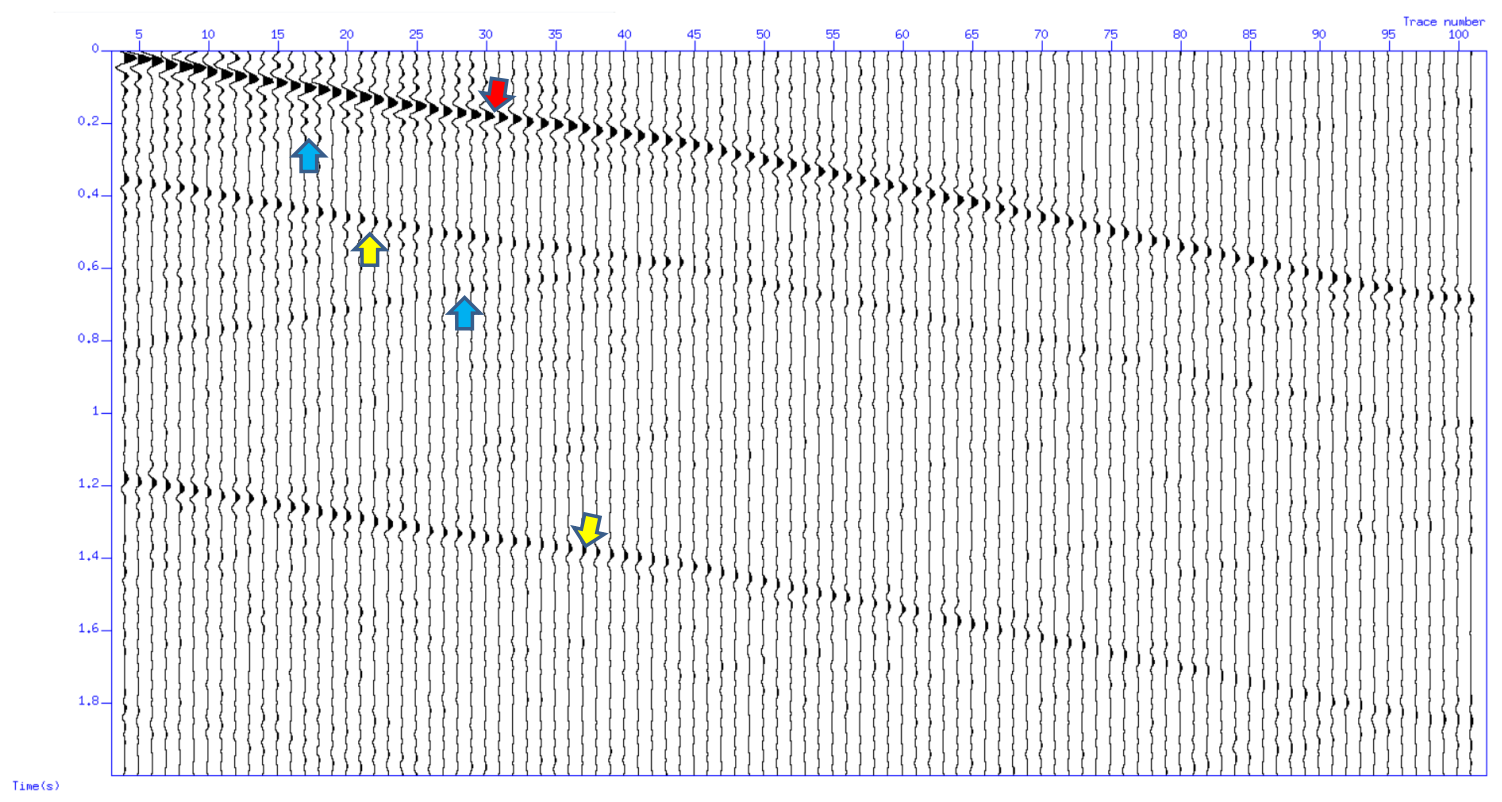

(b)

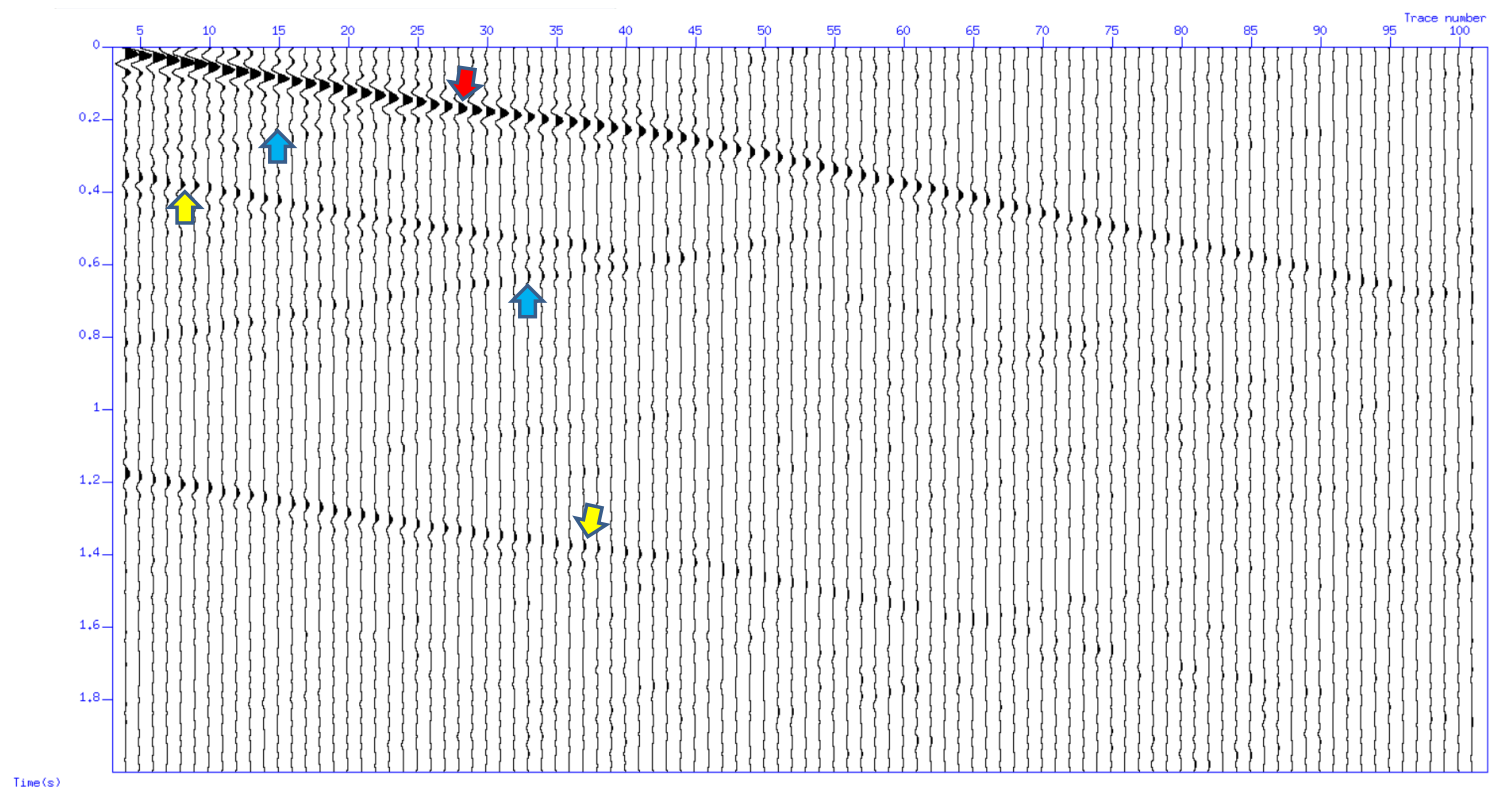


(c)

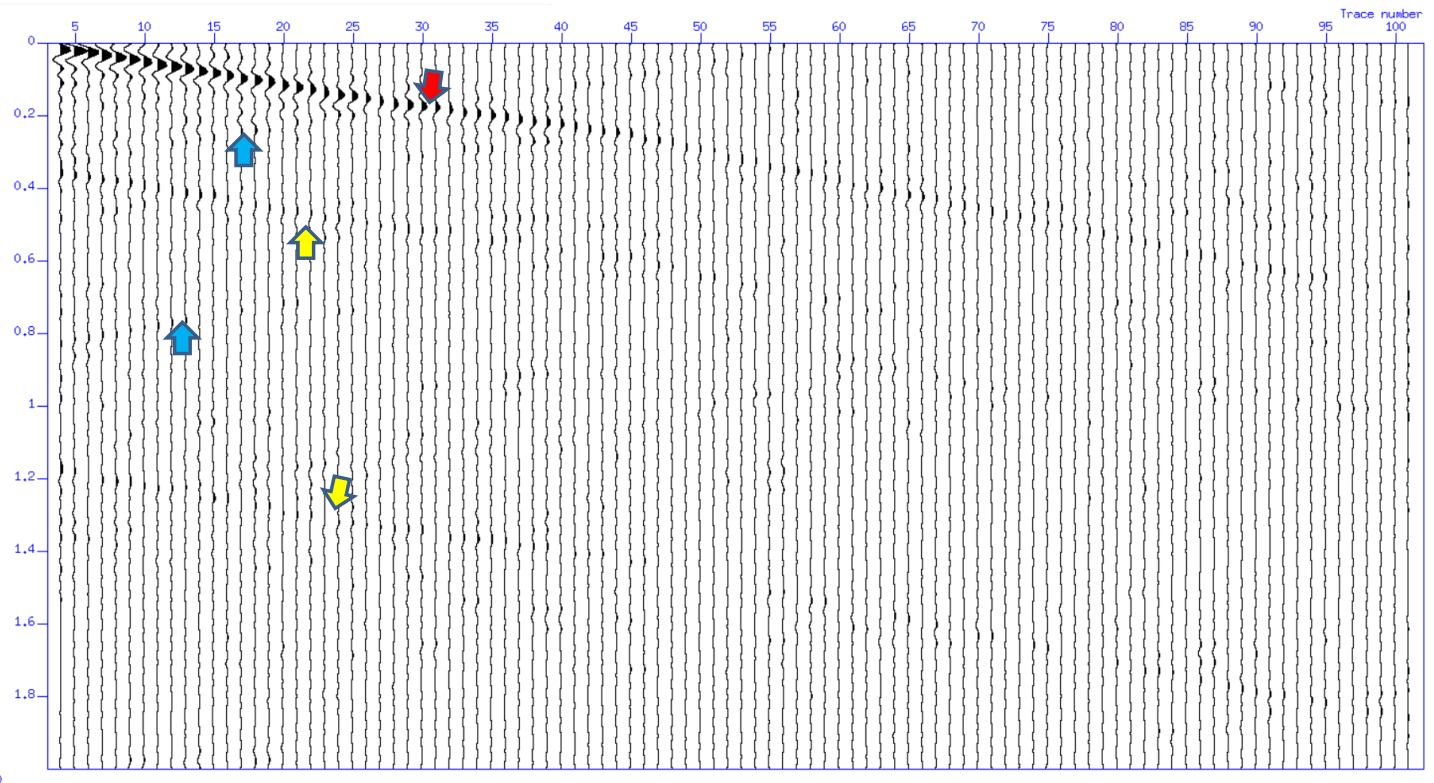

(d)

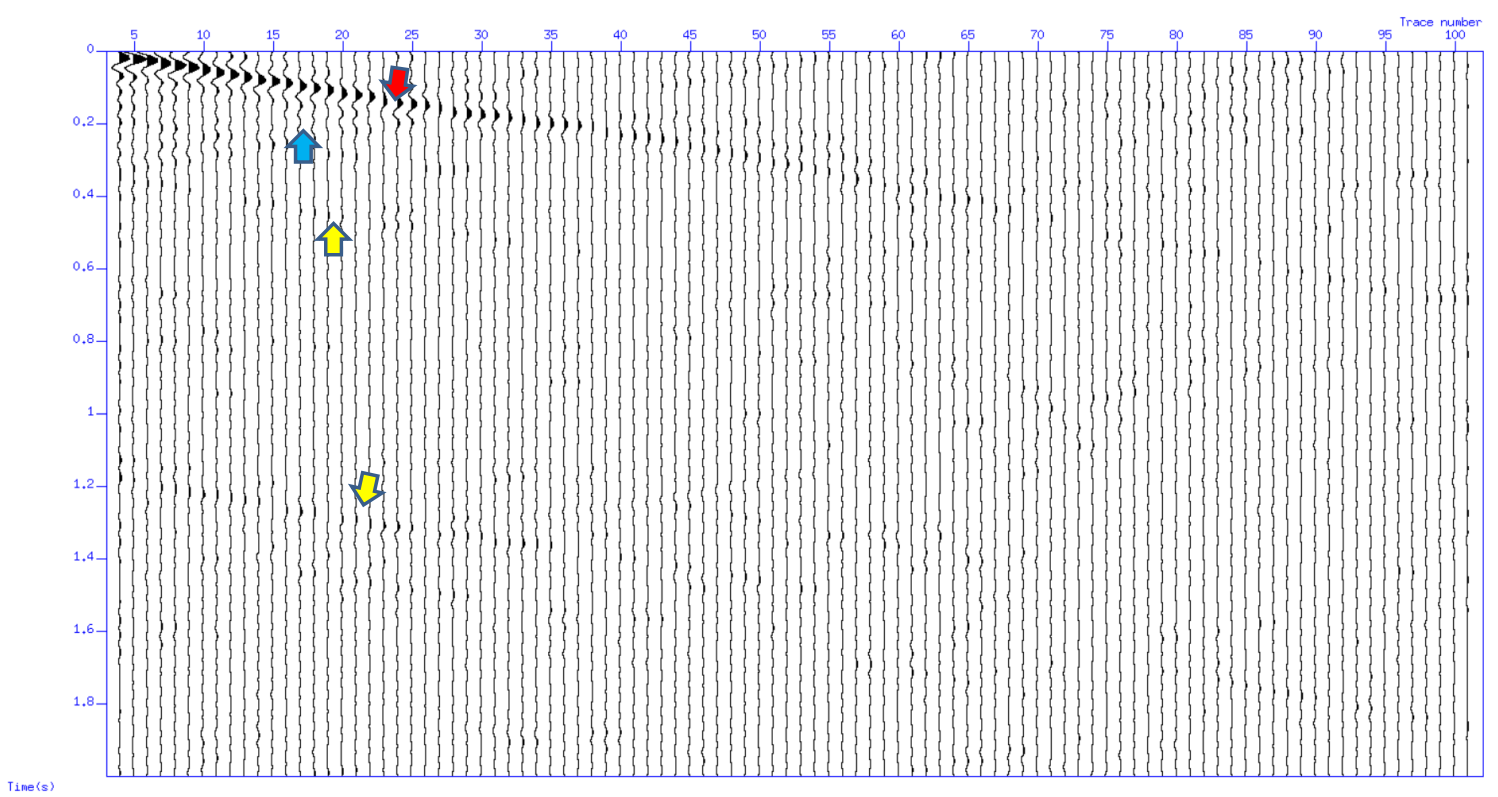

Figure 34. (a), (b), (c), and (d) show virtual VSP shot gather after cross-correlation for boundaries 1, 2, 3, and 4 for the anticline model using 500 sources, respectively. The red arrow shows downgoing primary wave or first-break curve, the yellow arrows show downgoing multiples, and the blue arrows show upgoing waves. 
(a)

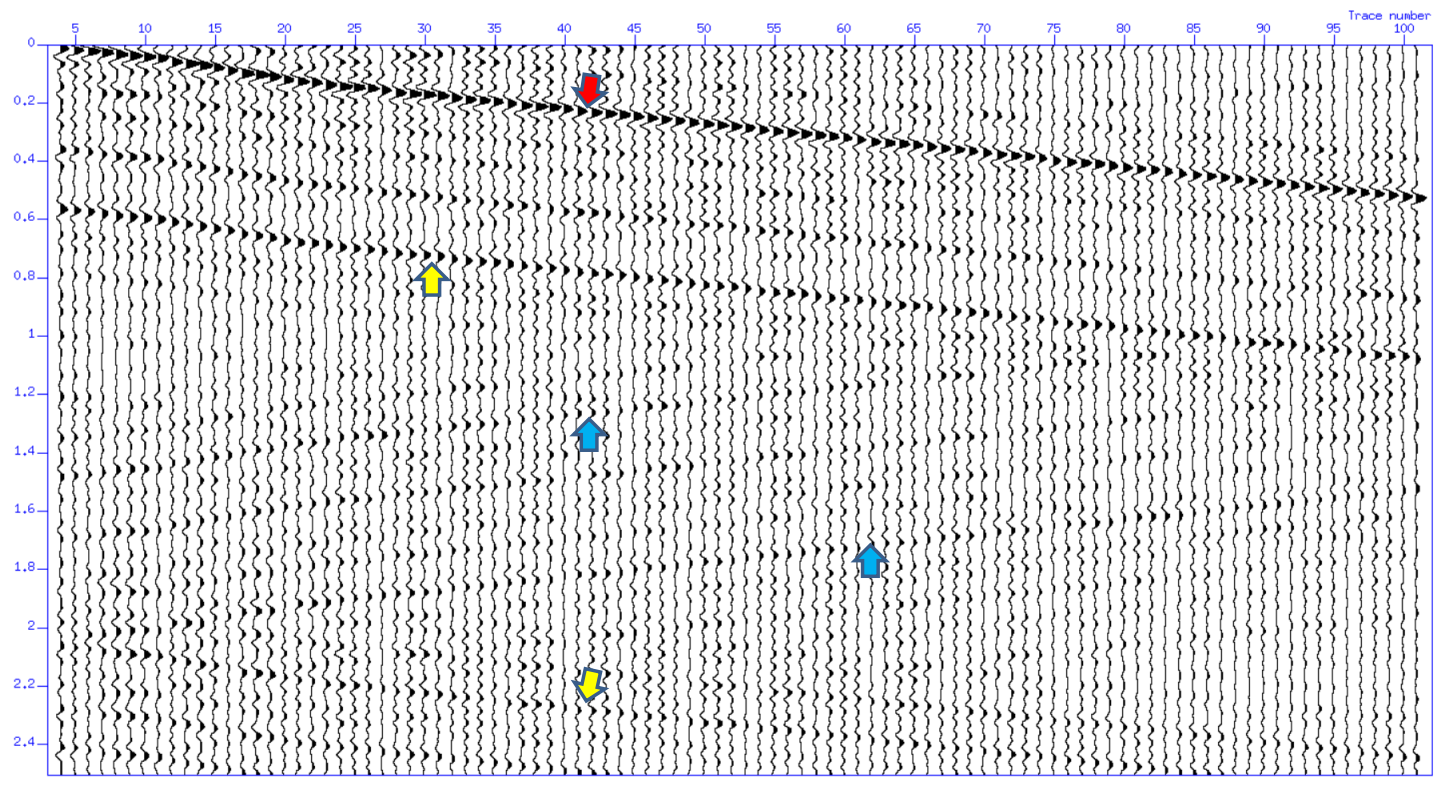

(b)

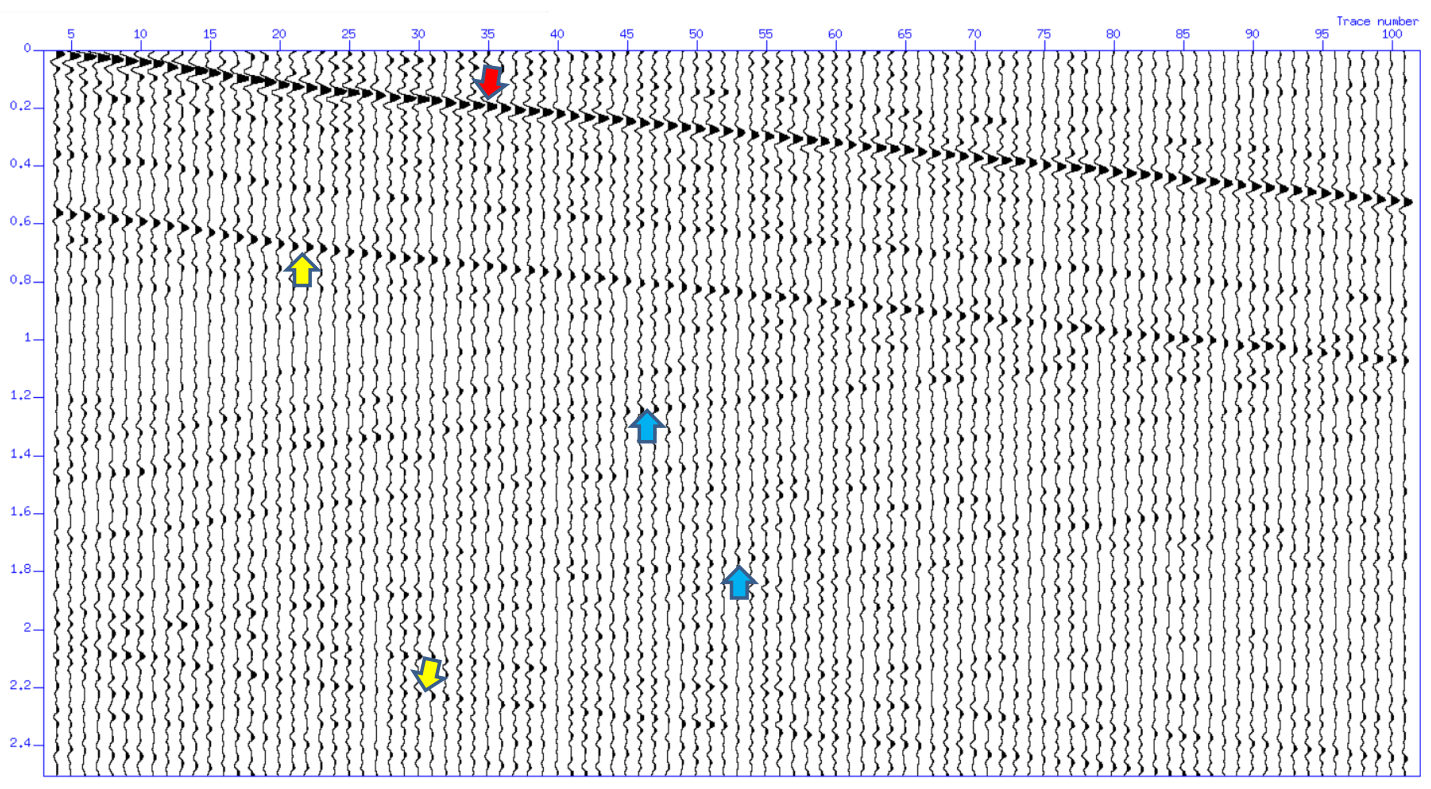


(c)

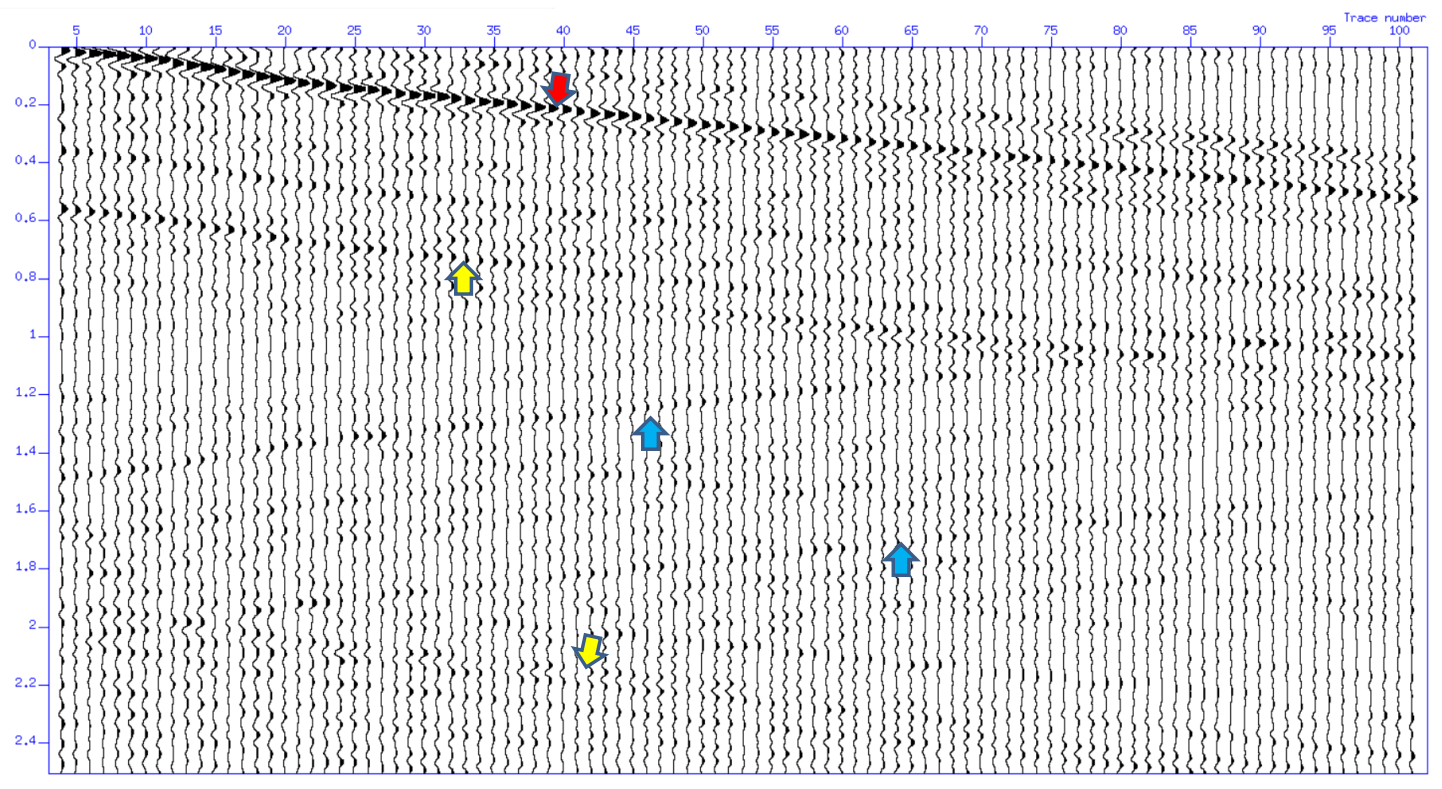

(d)

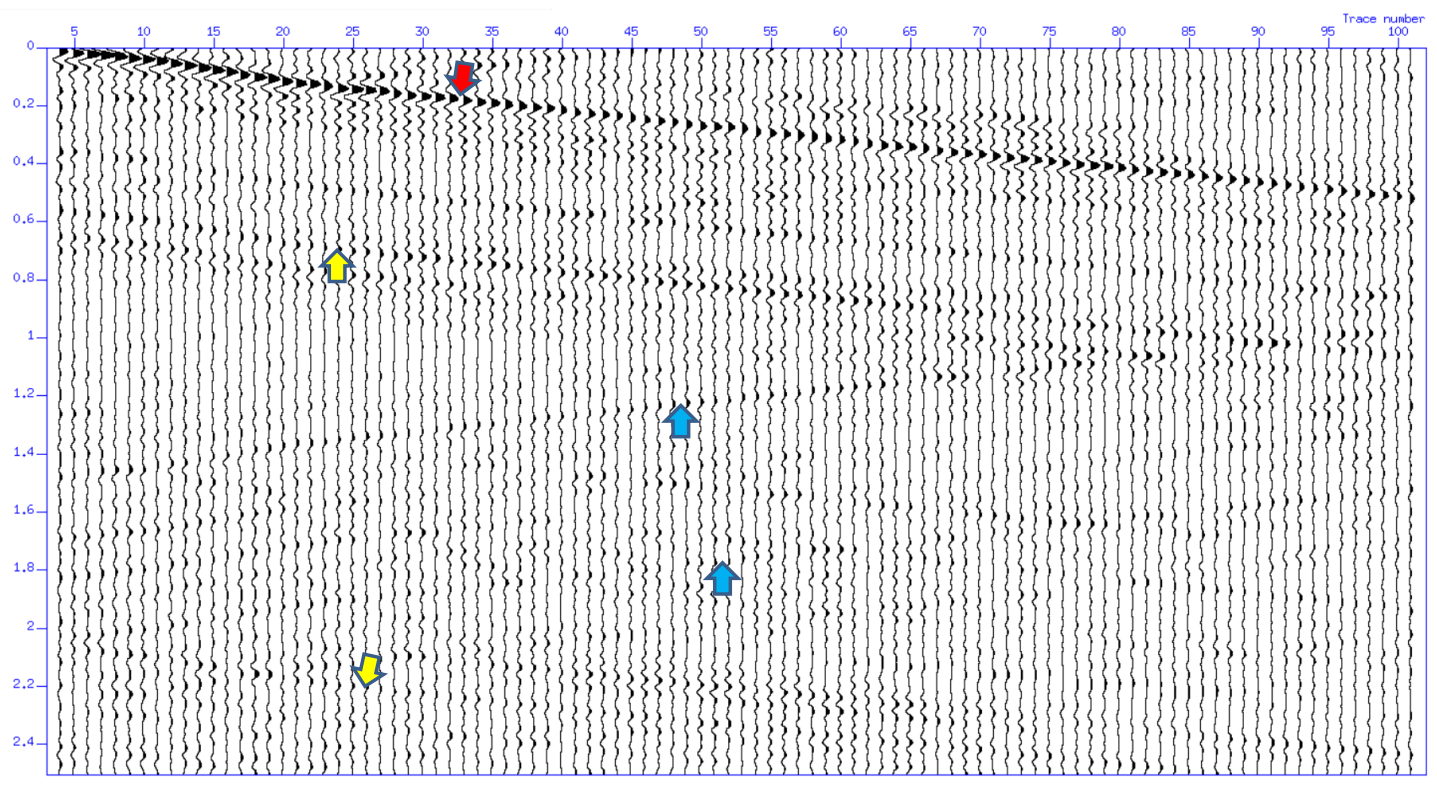

Figure 35. (a), (b), (c), and (d) show virtual VSP shot gather after cross-correlation for boundaries 1,2,3, and 4 for the anticline-syncline model using 5 sources, respectively. The red arrow shows downgoing primary wave or first-break curve, the yellow arrows show downgoing multiples, and the blue arrows show upgoing waves. 
(a)

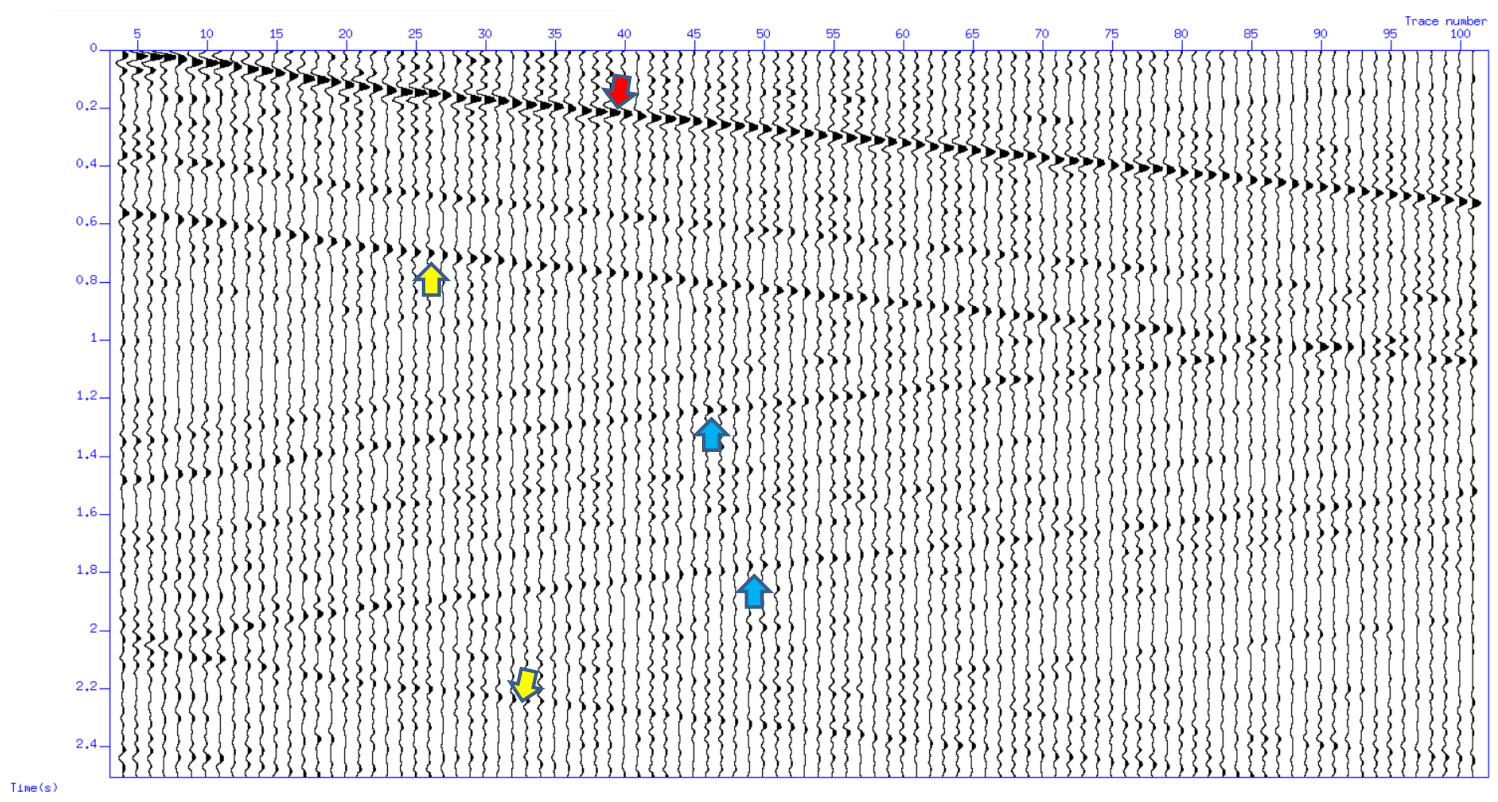

(b)

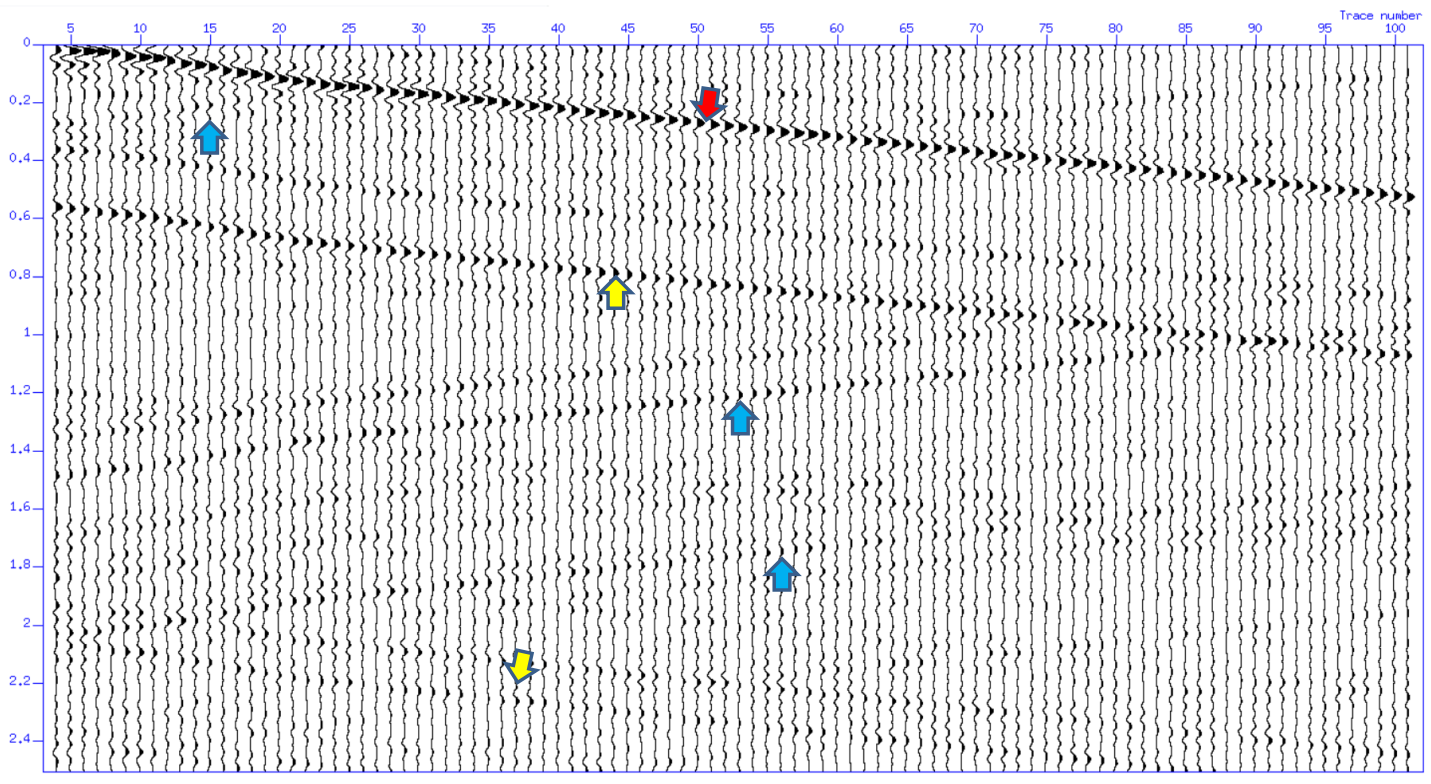


(c)

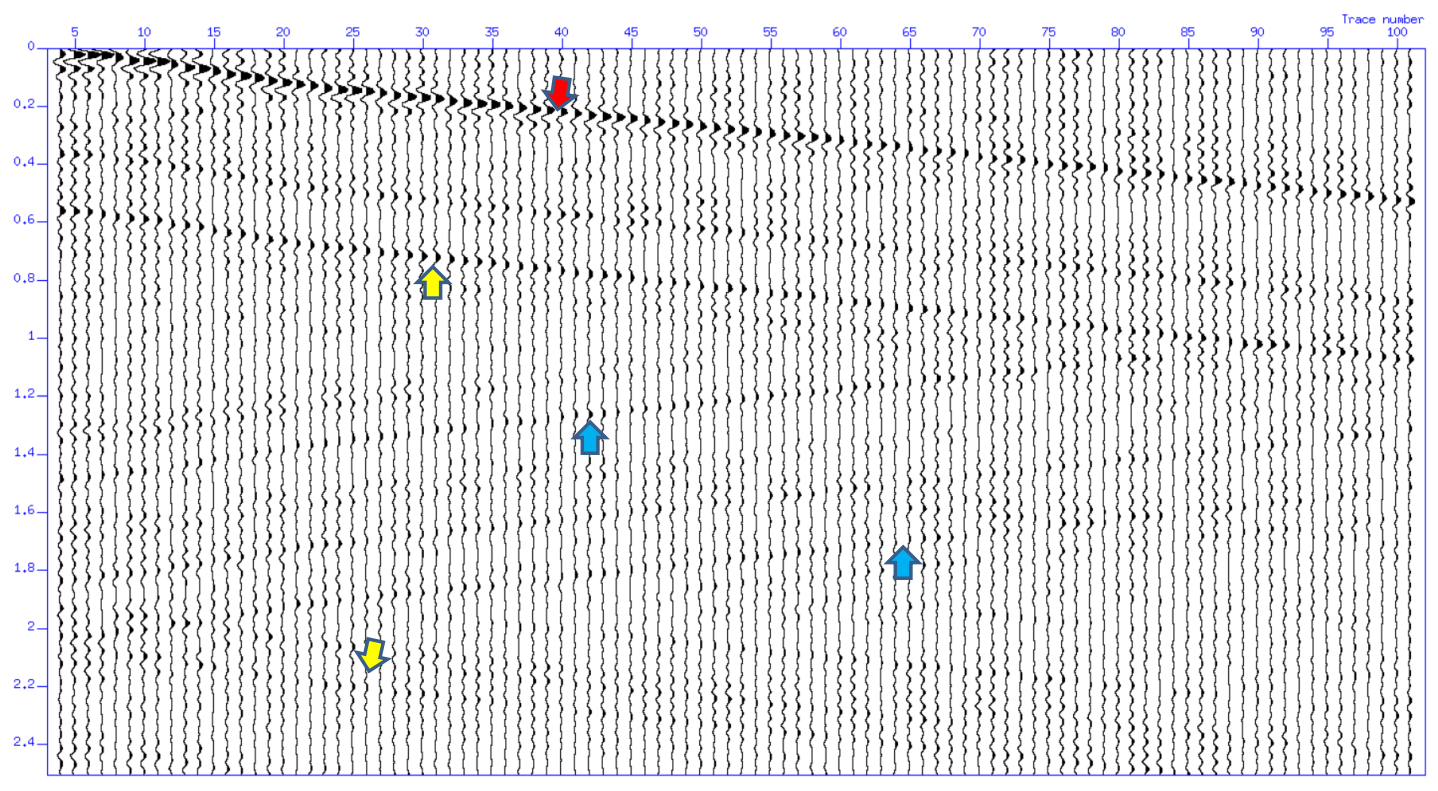

(d)

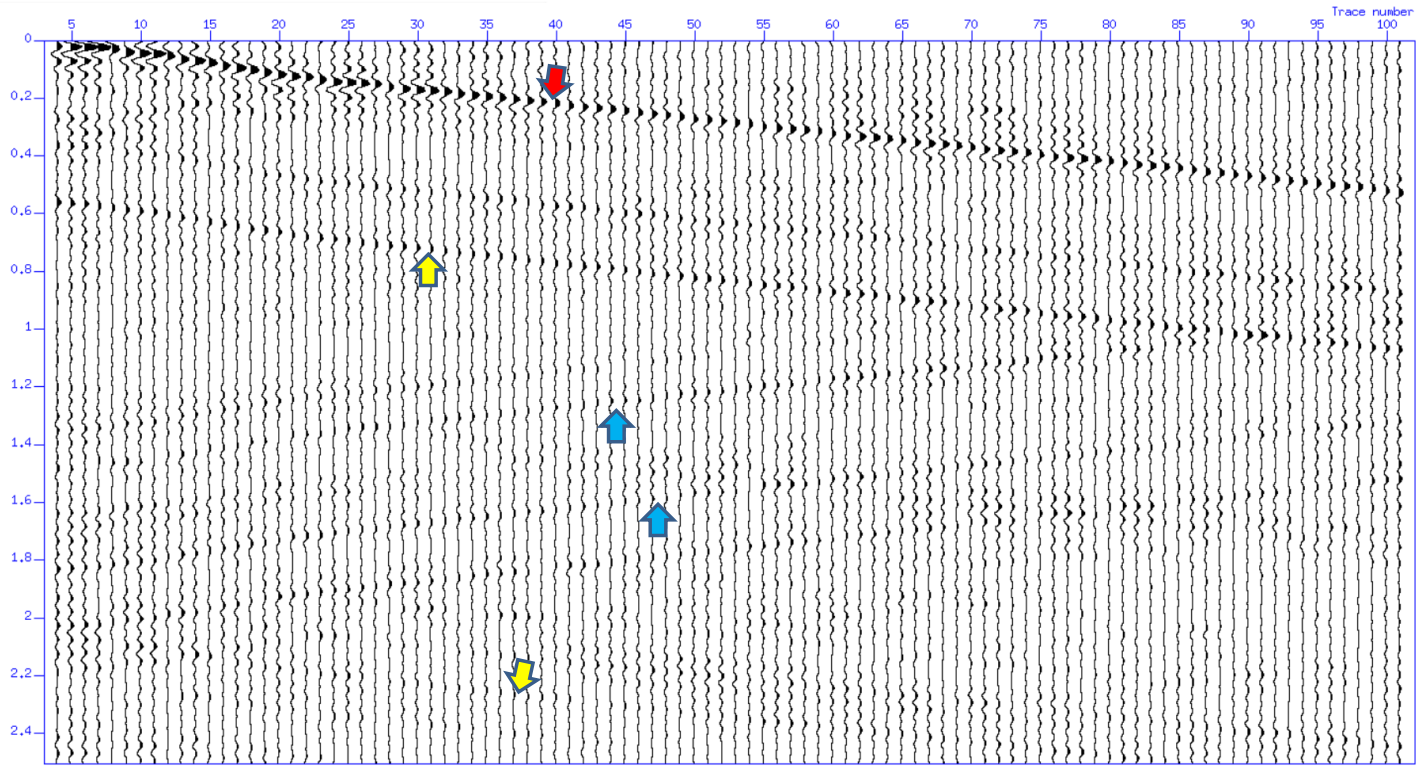

Figure 36. (a), (b), (c), and (d) show virtual VSP shot gather after cross-correlation for boundaries 1, 2, 3, and 4 for the anticline-syncline model using 20 sources, respectively. The red arrow shows downgoing primary wave or first-break curve, the yellow arrows show downgoing multiples, and the blue arrows show upgoing waves. 
(a)

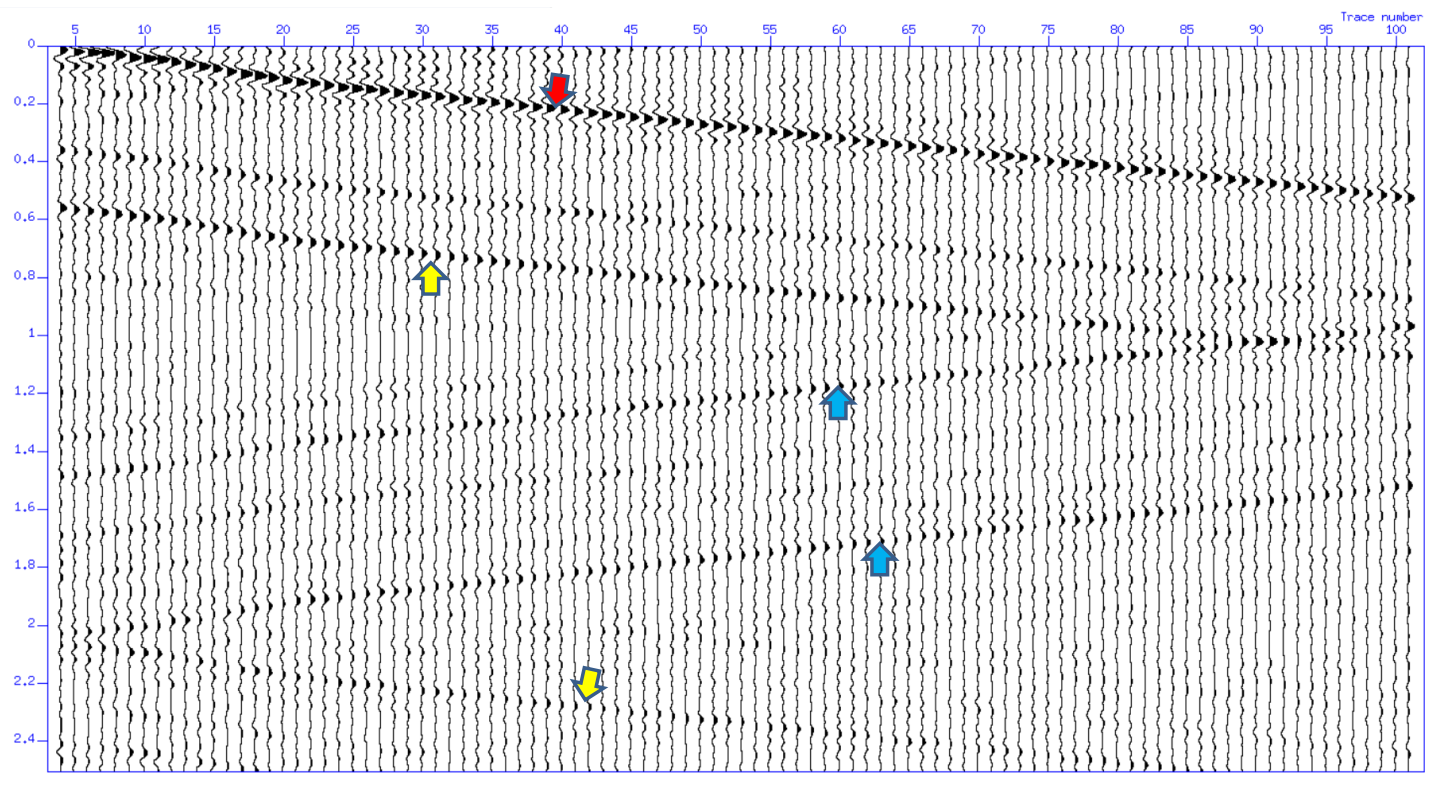

(b)

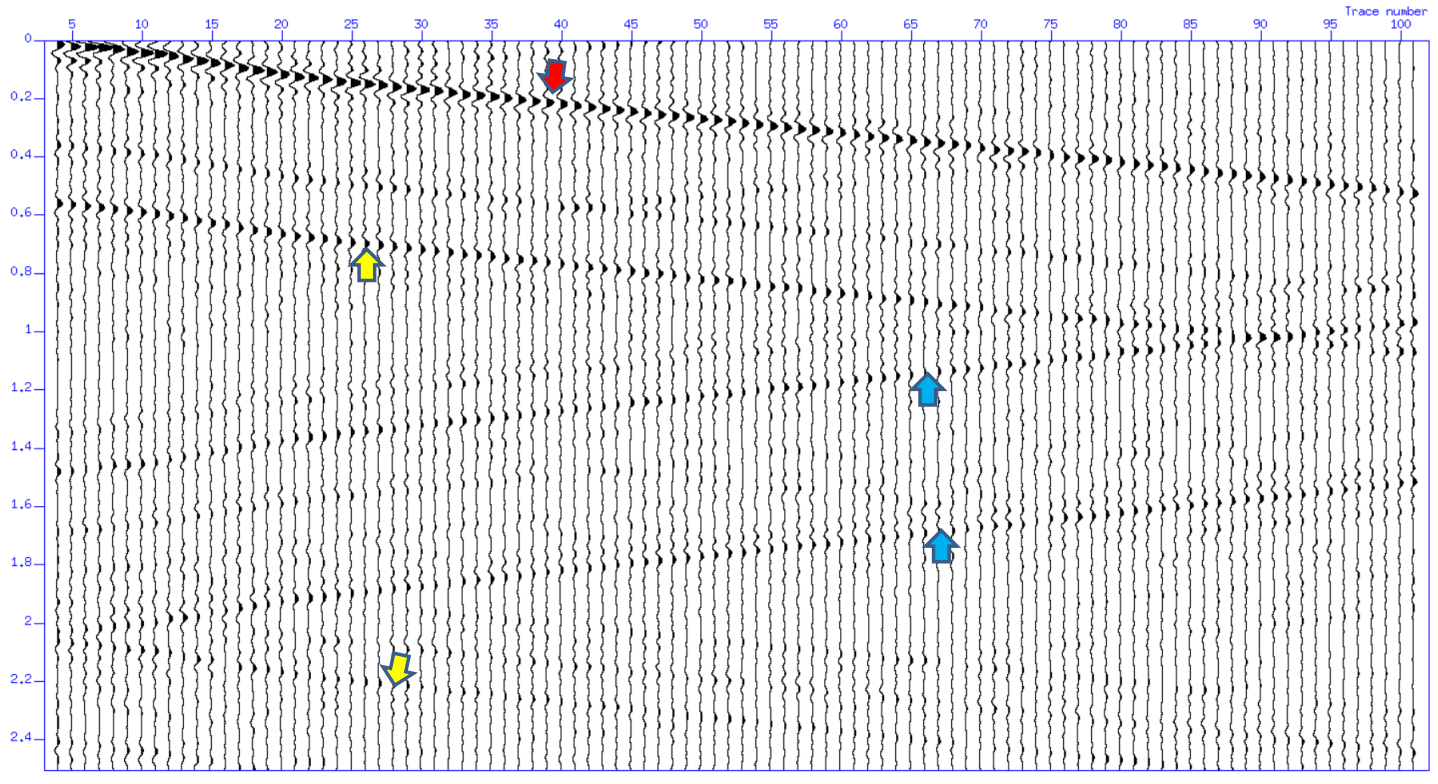


(c)

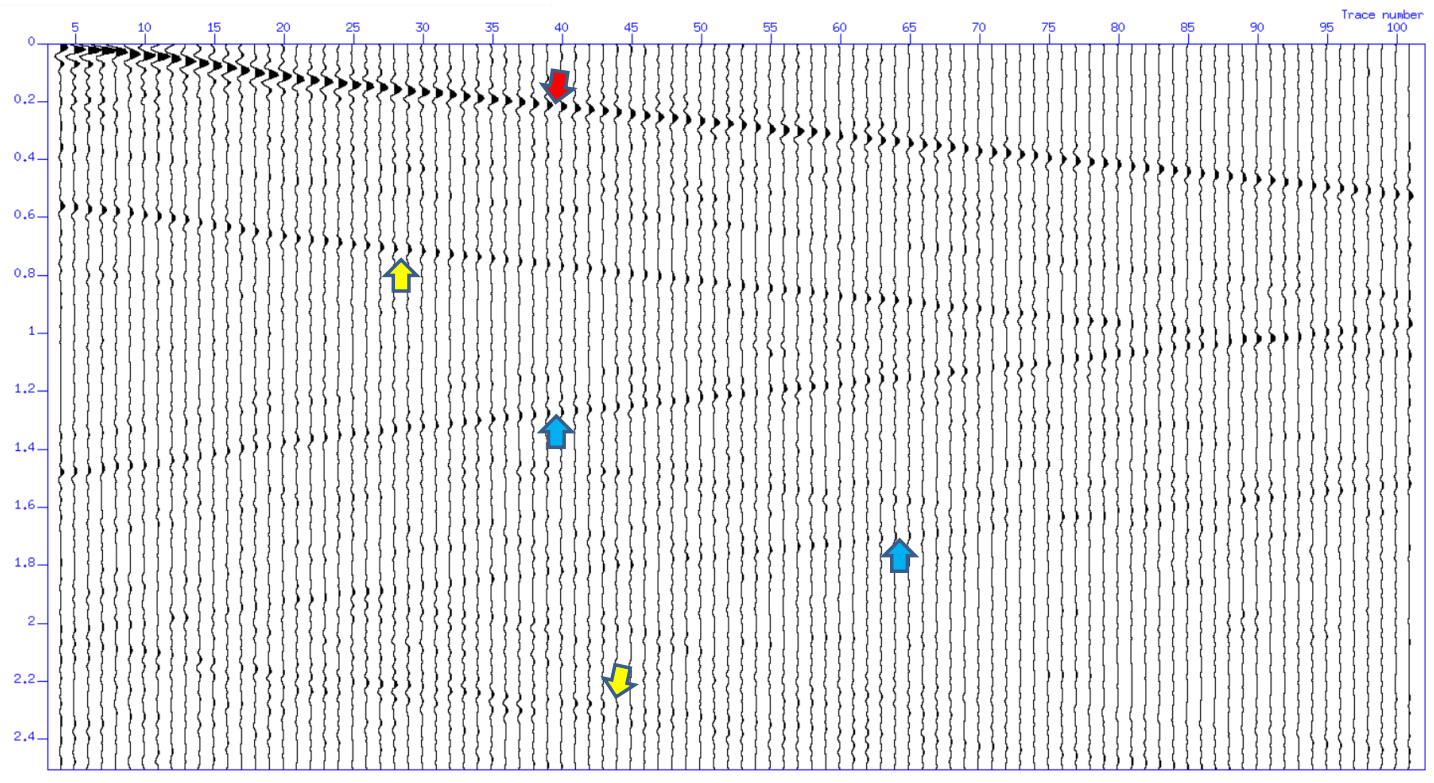

(d)

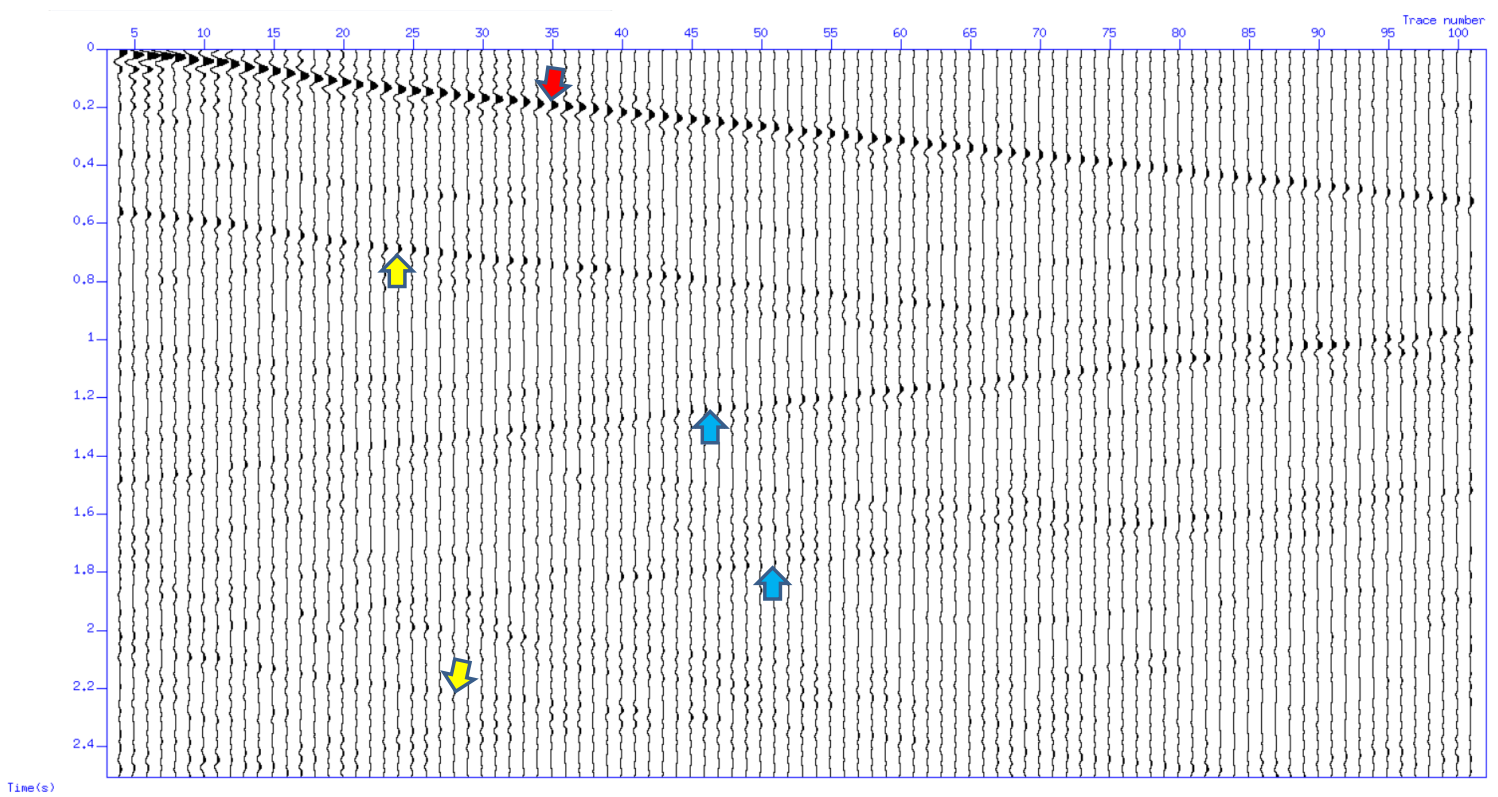

Figure 37. (a), (b), (c), and (d) show virtual VSP shot gather after cross-correlation for boundaries 1, 2, 3, and 4 for the anticline-syncline model using 500 sources, respectively. The red arrow shows downgoing primary wave or first-break curve, the yellow arrows show downgoing multiples, and the blue arrows show upgoing waves. 
After getting the 36 virtual VSP shot gather, I performed some processing steps for zero offset VSP acquisition. In the first step, I picked maximum energy center points of traces corresponding to maximum amplitude on downgoing primary trend because the traces in the VSP data I obtained after cross-correlation contain zero-phase wavelets. In Figure 38, Figure 38 a shows boundary 1 containing 20 sources in the flat model as an example. In the example data, the red line shows downgoing primary, yellow arrows show downgoing multiples, and blue arrows show upgoing waves or reflections. The rectangular area in Figure 38a shows the traces between 21 and 31 closely how to pick the arrivals' central energy points closely in Figure 38b.

(a)

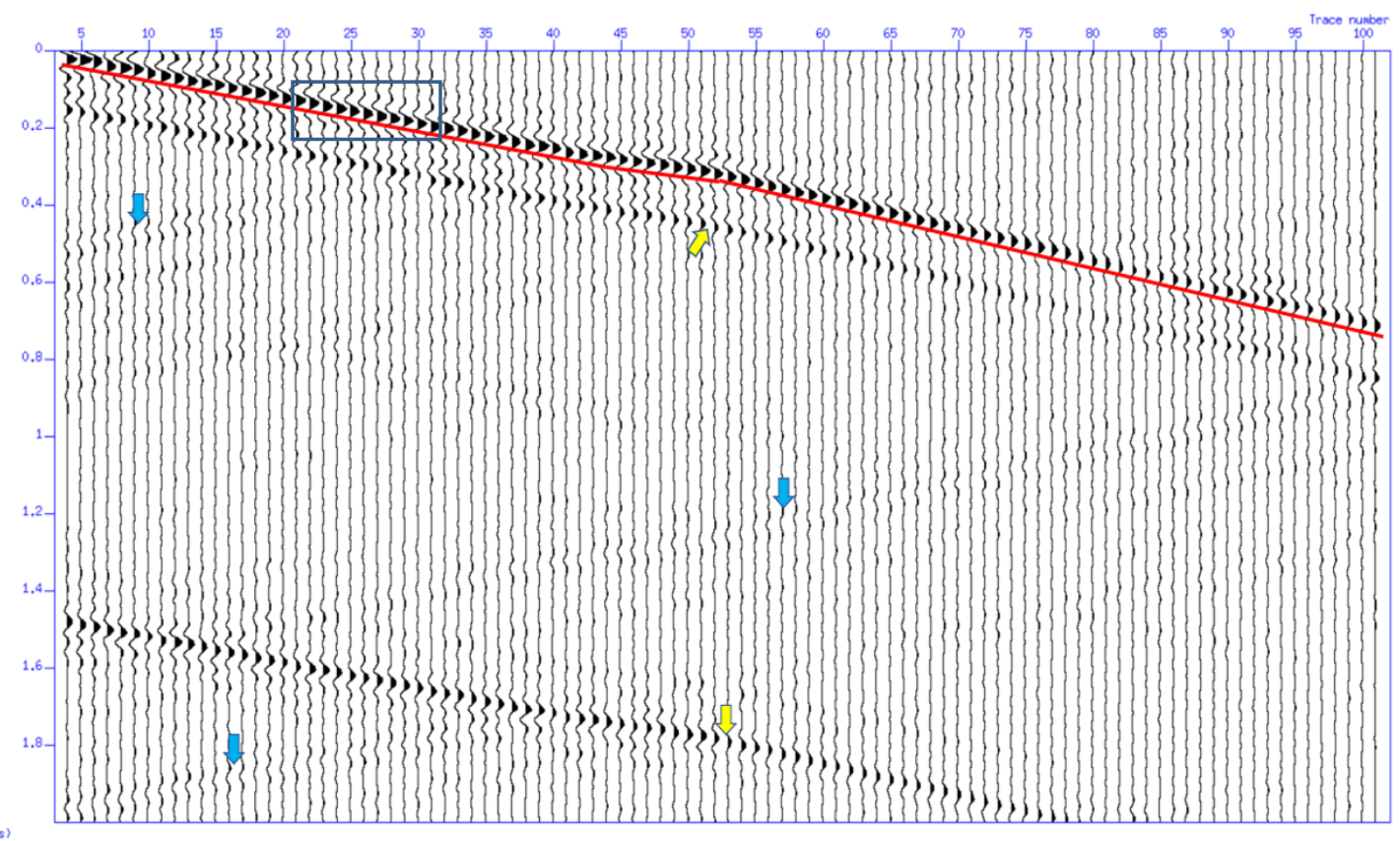


(b)

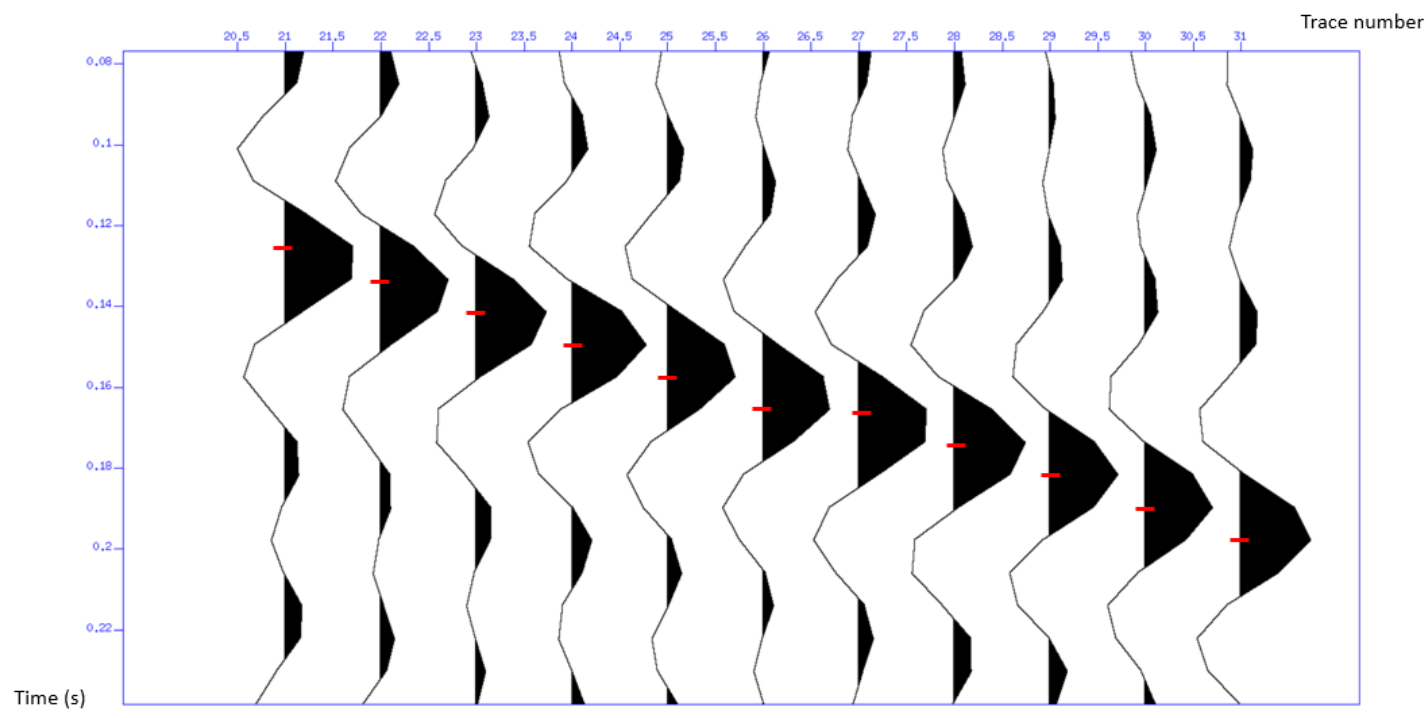

Figure 38. (a) shows the VSP data containing 20 sources for boundary 1 in the flat model. The red line shows downgoing primary, the yellow arrows show downgoing multiples, and the blue arrows show upgoing waves or reflections. (b) shows the rectangular section closer. This section was chosen as an example in terms of showing the central points of maximum energy (red dashes) picked on the traces between the 21 st and 31 st traces. $x$-axis shows trace numbers while $\mathrm{y}$-axis shows time (s).

After picking the traces, I set up the geometry to my data by loading the times picked, geophone interval, and depth values of the geophones to the header, which start from 1,000 $\mathrm{m}$ to $3,000 \mathrm{~m}$, in the borehole. Figure 39 shows this data that is loaded the geometry duration of 2 seconds. The red line shows downgoing primary wave, the yellow arrows show downgoing multiples, and the blue arrows show upgoing waves.

In the second step of the VSP processing, since the VSP data contains downgoing wavefield, as well as upgoing wavefield and they have different slopes, and they interfere with each other, these wavefields must be separated from each other. For the separation of the wavefields, two methods are used commonly. They are median filtering and frequencywavenumber filtering. I used frequency-wavenumber filtering. For this, the data is taken from the time domain to the frequency domain by 2D Fourier Transform. The downgoing wavefield has a negative slope and is located in the positive quadrant while the upgoing wavefield has a positive slope and is located in the negative quadrant. With frequencywavenumber filtering, the upgoing wavefield is separated from the downgoing wavefield (Hardage, 1985). Figure 40 shows the separation of the upgoing wavefield from the downgoing wavefield. 
depth (m)

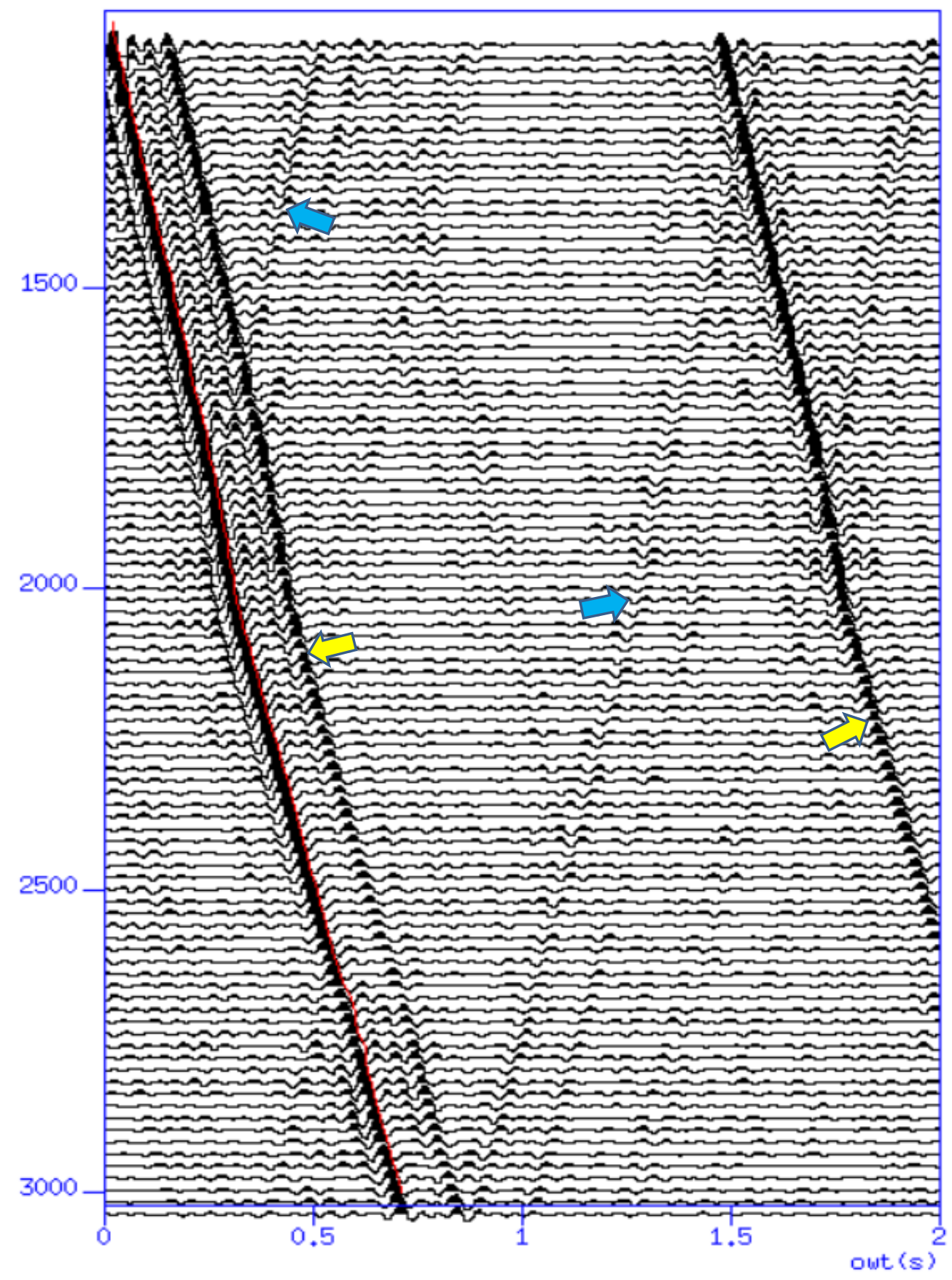

Figure 39. The VSP data obtained by adding geophone depth values in the borehole. The red line shows the picked traces in Figure 38a. The red line shows downgoing primary wave, the yellow arrows show downgoing multiples, and the blue arrows show upgoing waves. 

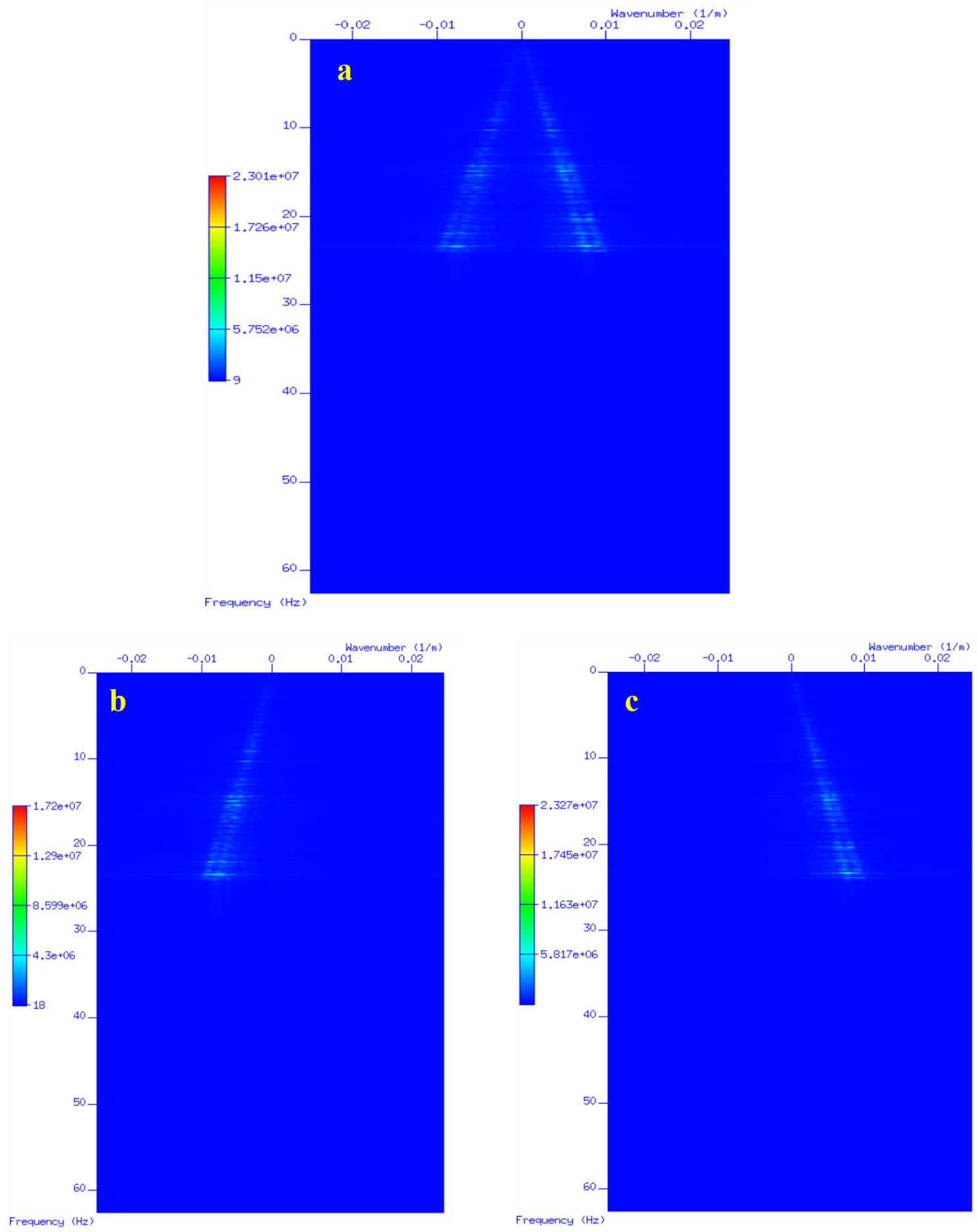

Figure 40. Separation of the downgoing wavefield and the upgoing wavefield. (a) shows before frequency-wavenumber filtering. (b) shows the upgoing wavefield positioned in the negative quadrant. (c) shows the downgoing wavefield positioned in the positive quadrant. The data that was used in the fdelmodc for VSP was $24 \mathrm{~Hz}$, which can be seen in the Figure. $\mathrm{x}$-axis shows wavenumber while the $\mathrm{y}$-axis shows frequency. 
After filtering the downgoing wavefield, both wavefields have been obtained with a timedepth figure (Figure 41 and Figure 42). As can be seen, the downgoing and upgoing that coincides are separated from each other successfully by frequency-wavenumber (f-k) filtering.

In the next step, all traces in the VSP data are shifted using the first arrival time. Therefore, upgoing events reduced to the time they can be recorded. Thereby, all traces in the upgoing wavefield are aligned and flattened (Figure 43). Thus, one-way time is transformed into two-way travel time (TWT).

The last step is stacking the traces. The flattened upgoing wavefield is used for building corridor stacks, which can be used to compare with the results of the surface seismic acquisition. There are two issues here. One is using the inside corridor stack, which contains primary events and multiples. The second is using the outside corridor stack, which is multiple-free and has only primary events. I used the outside corridor stack, containing 10 traces inside of the red region (Figure 44). Note that the one-way time (owt) is transformed into two-way travel time (TWT).

After zero-offset processing steps, I combined surface seismic data and VSP data (Figure 45). To look closely, I spliced took 10 VSP traces that have been obtained by processing into 10 seismic traces that are just behind the 10 VSP traces (Figure 46). 
$\operatorname{depth}(m)$

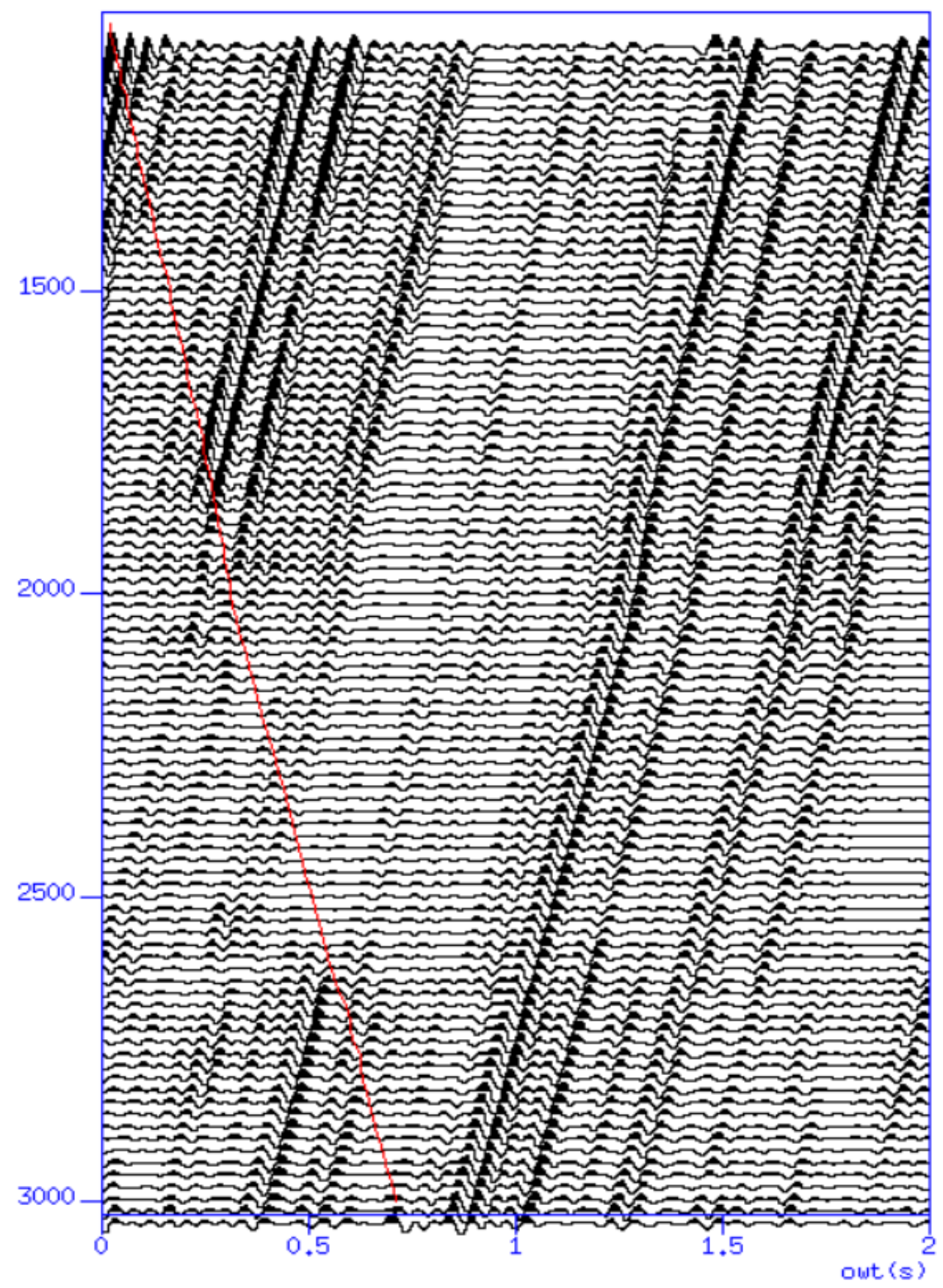

Figure 41. The separated upgoing wavefield. The red line shows the P-wave downgoing primary trend in Figure 39. 
$\operatorname{depth}(m)$

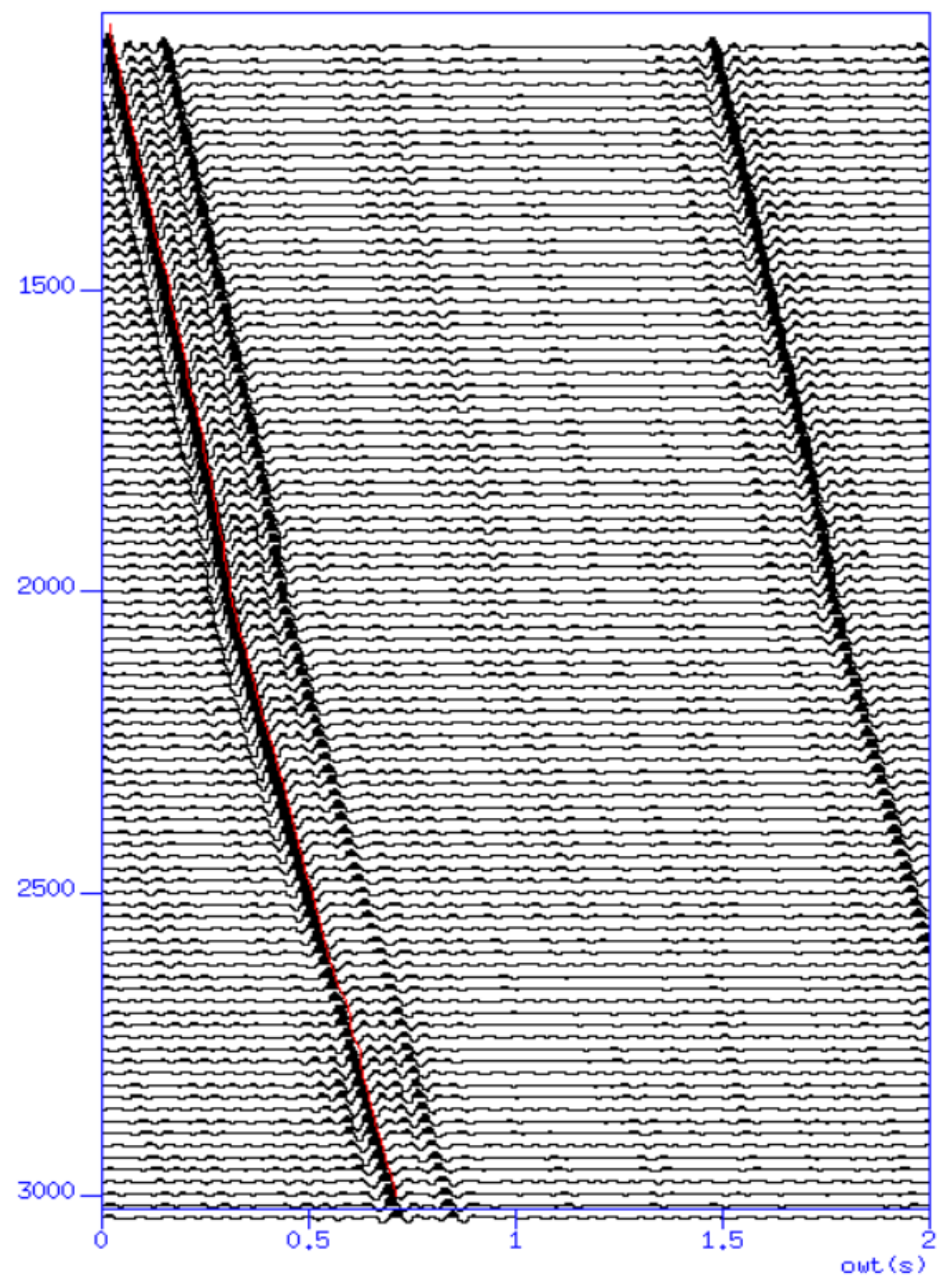

Figure 42. The separated downgoing wavefield. The red line shows the P-wave downgoing primary trend in Figure 39. 


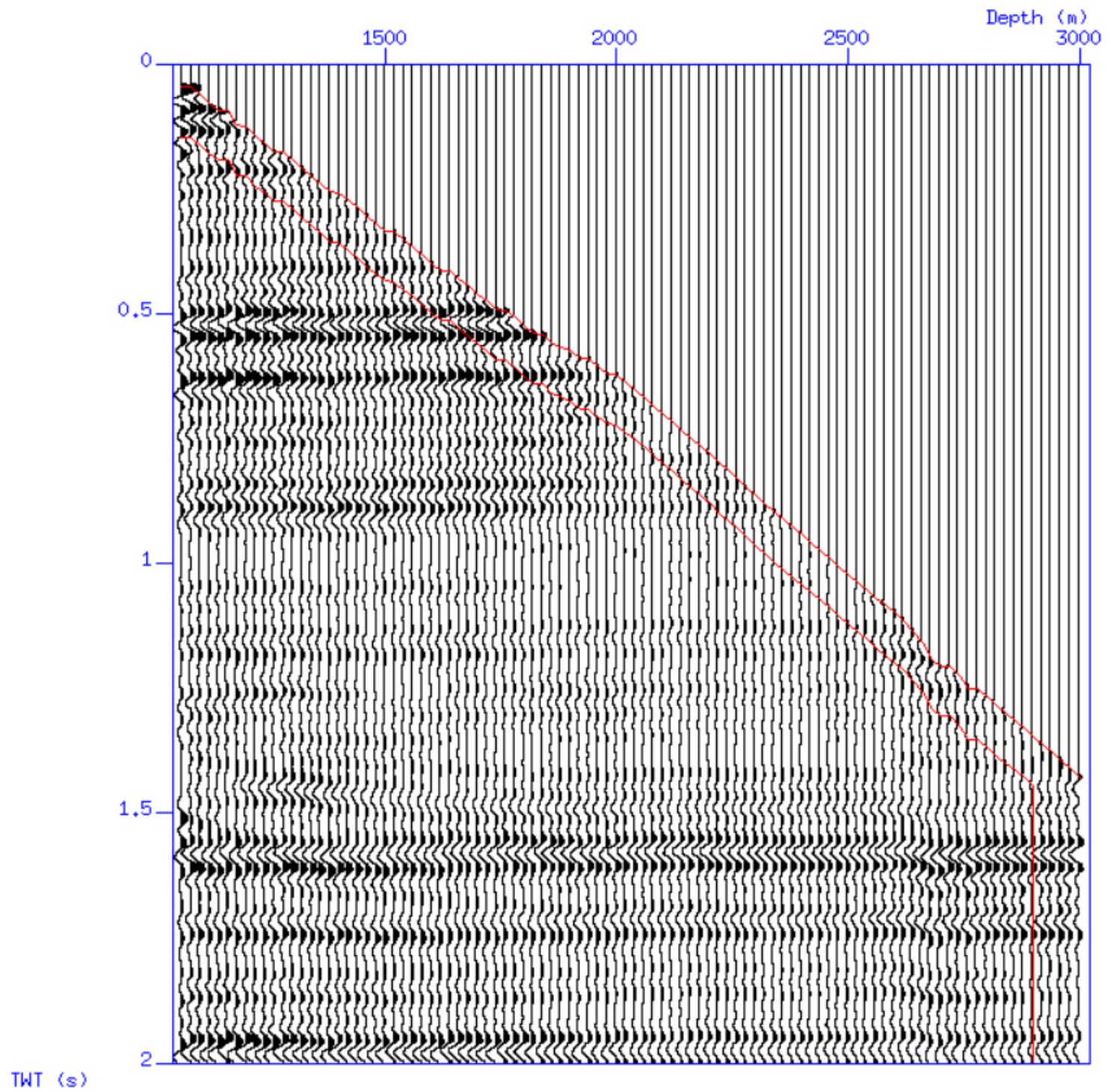

Figure 43. The flattened upgoing wavefield. The red narrow corridor corresponds to the outside corridor stack, containing 10 traces. The receivers on the VSP in the borehole are starting from $1,000 \mathrm{~m}$ to $3,000 \mathrm{~m}$. 


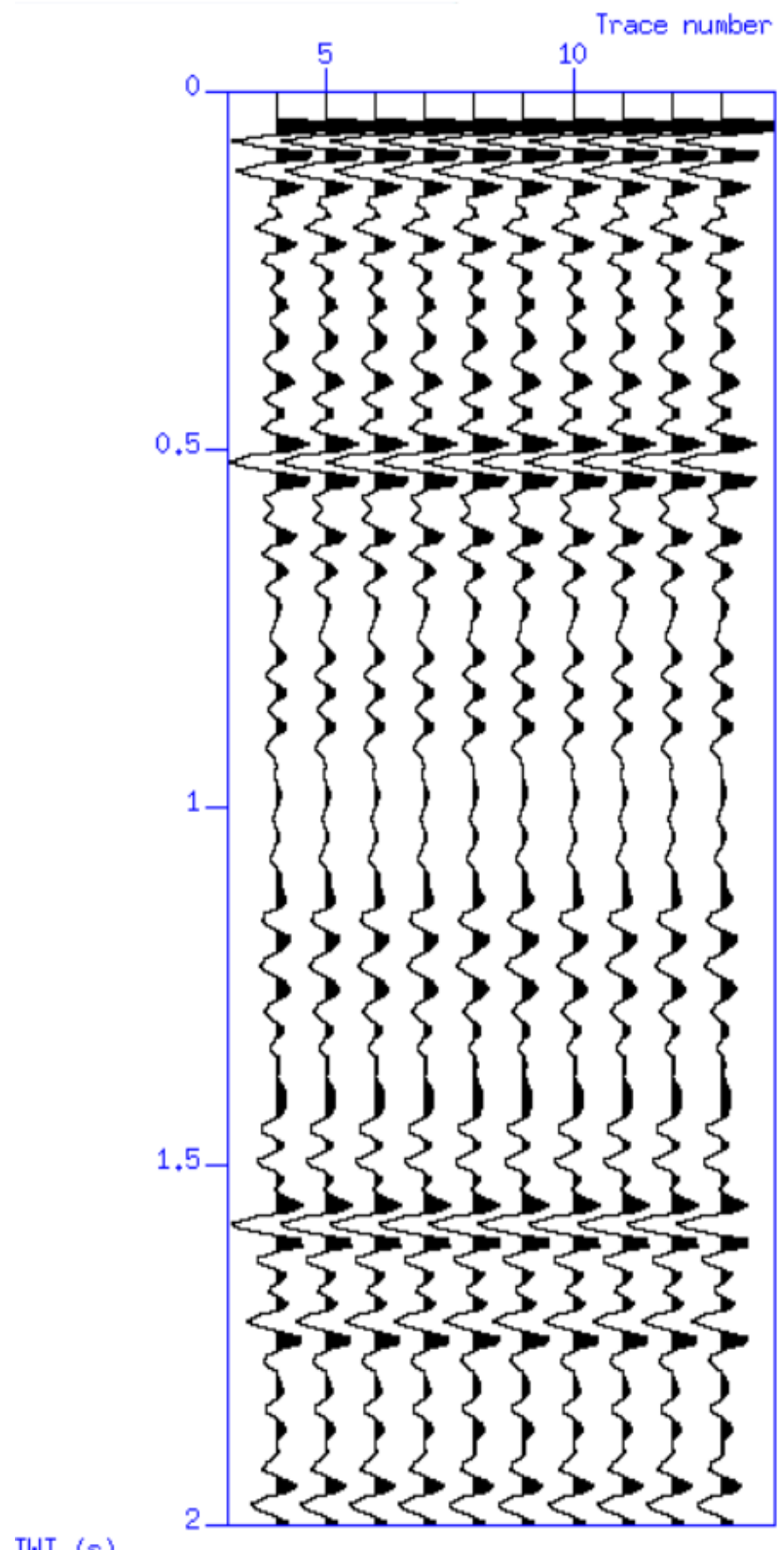

TWT (s)

Figure 44. The corridor stack that consists of 10 traces corresponding to the red narrow corridor in Figure 43. 


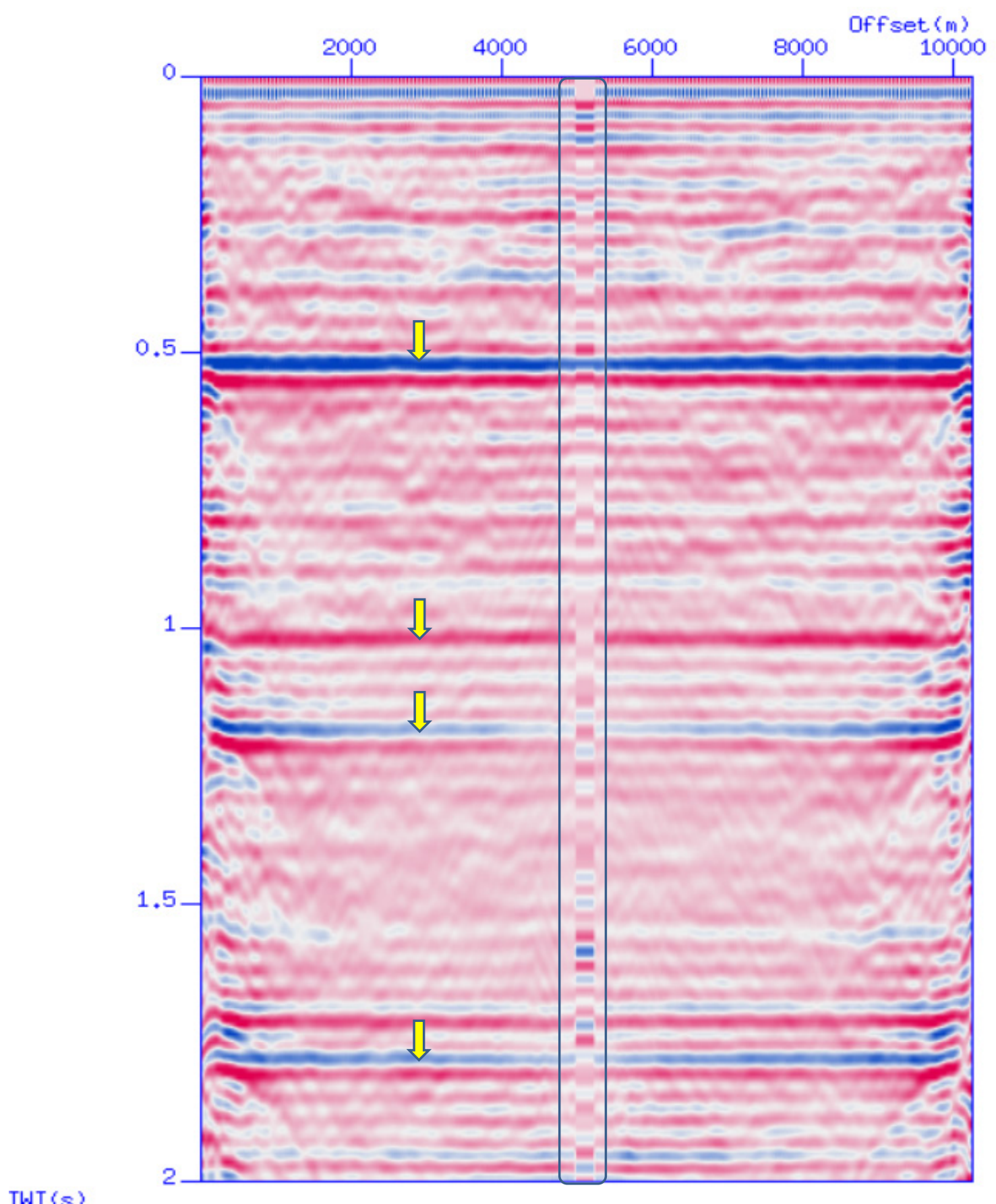

Figure 45. The combined surface seismic and zero-offset VSP data. The yellow arrows show the reflections from interfaces in the surface seismic flat model. The rectangular area in the middle corresponds to the 10 traces of the zero-offset VSP positioned offset between $5,025 \mathrm{~m}$ and $5,250 \mathrm{~m}$ on the surface. 


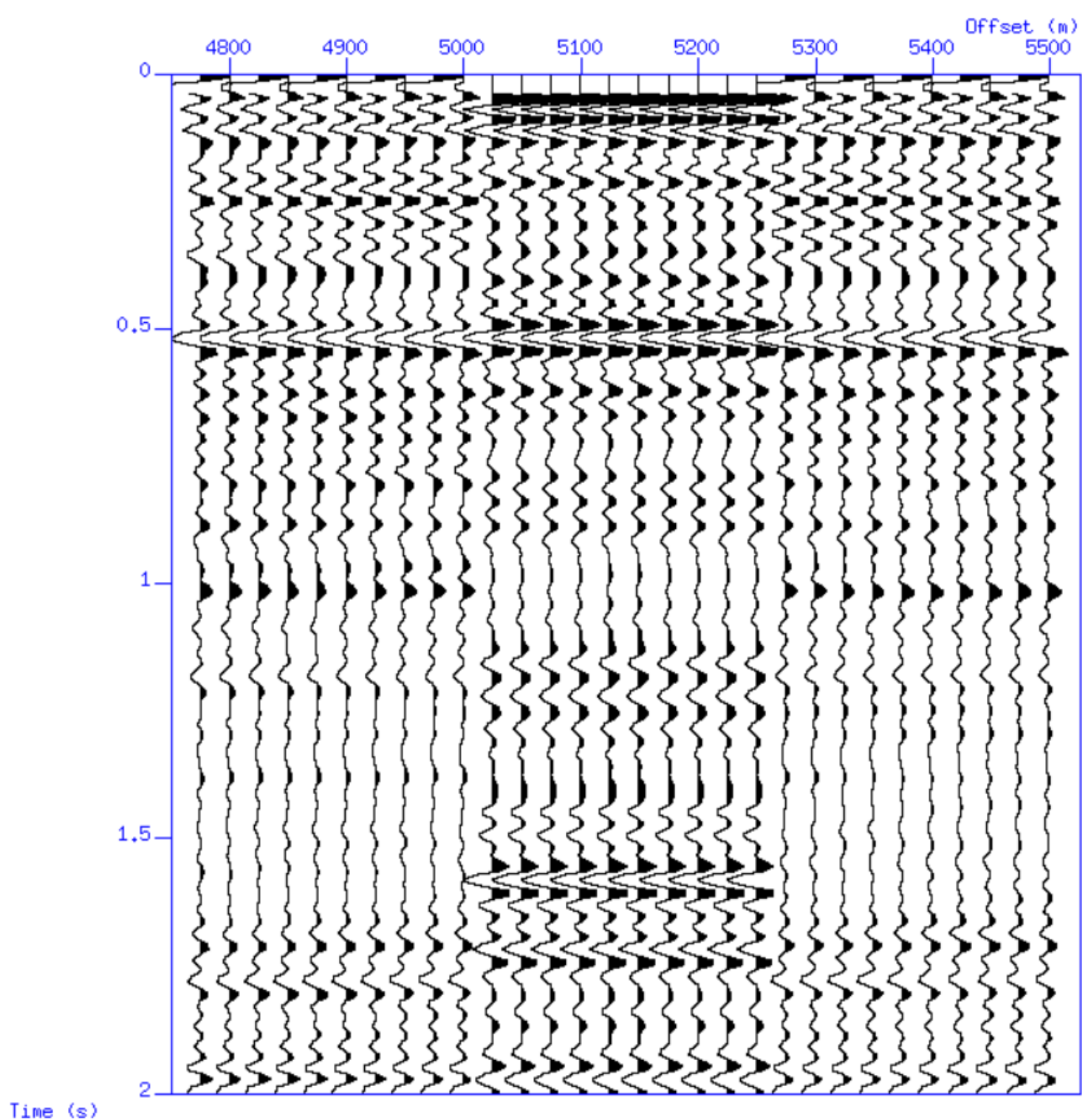

Figure 46. The wiggle Figure of splicing the corridor stack into the surface seismic between $5,025 \mathrm{~m}$ and 5,250 $\mathrm{m}$ offset. 


\subsection{Synthetic Seismogram}

A synthetic seismogram is an important tool that provides the link between well and seismic data. Thanks to synthetics, seismic interpretation becomes easier. By combining seismic, VSP, and synthetic, events corresponding to geological structures in the seismic data can be detected more easily. The events that are not seen in the surface seismic can be detected. To produce a synthetic seismogram, a model that consists of velocities and densities is required. Then, with the help of the velocities and densities, the acoustic impedances are calculated first. After, the series of reflection coefficients are calculated by acoustic impedances. To produce a synthetic seismogram, the source wavelet is convolved with this reflection coefficient series.

As the last step of this thesis, I used and developed a ready code that generates synthetic seismograms using MATLAB software to determine whether VSP or seismic is better in determining the coal layer (the region marked with yellow dashes in the models) and see whether factors such as the number of sources and noise boundaries are effective in this detection or not. In this code, I used Ricker or zero-phase wavelet as the source wavelet. Before comparison, I applied a geometric spreading correction to all seismic and VSP data because the wave amplitude decays on the wavefront when the expansion of the wavefront. I also applied automatic gain control to amplify the reflections from deeper formations. Then, I compared 10 traces for seismic, VSP, and synthetic. 


\section{Results}

By applying the cross-correlation method for the surface seismic and the zero-offset VSP methods, virtual shot gathers corresponding to the different number of sources and noise boundaries for three different models have been obtained. Figures 15-16-17 show the seismic virtual shot gathers obtained for flat model, anticline model and anticline-syncline model. For the surface seismic, zero-offset stacked sections were obtained as a result of the required data processing steps. Figures 19-20-21 show flat model results, Figure 22-23-24 show anticline model results, and Figure 25-26-27 show anticline-syncline model results, respectively.

For the VSP method, virtual shot gathers are shown in Figure 29-37. Figures 29-30-31 show flat model results, Figures 32-33-34 show anticline model results, and Figures 3536-37 show anticline-syncline model results for virtual VSP shot gathers.

After the data processing steps for surface seismic and zero-offset VSP, the surface seismic and zero-offset VSP were combined and 10 traces were selected. With the help of MATLAB software, 10 synthetic traces were also produced and two methods were compared each other.

Figures 47-58 show the retrieved surface seismic and combined with VSP, the seismic seismogram, VSP seismogram, and synthetic seismogram comparison for boundary 1,2 , 3 , and 4, which has 5, 20, and 500 sources for the flat model, respectively. The reflections from four interfaces are seen in $0.53 \mathrm{~s}, 1.03 \mathrm{~s}, 1.19 \mathrm{~s}$, and $1.79 \mathrm{~s}$ for flat model, 12 results in total. In these figures, the yellow arrow shows the reflection from the coal layer, and the red rectangular boxes show the reflections from interfaces.

According to the flat model results, the surface seismic method is more successful to solve the coal layer than the zero-offset VSP method (the 2 nd red rectangular box in (c) in Figures 47-58). The surface seismic method also solves the other reflections better than the zerooffset VSP method. Both methods sometimes have been unsuccessful to solve the fourth reflection (Figure 47-48-49-50). We also see that when the number of source increase, meaning higher signal/noise ratio, surface seismic starts solving the reflections from the 4th interfaces, gradually (in (c) in Figures 51-58).

Figures 59-70 show the retrieved surface seismic and combined with VSP, the seismic seismogram, VSP seismogram, and synthetic seismogram comparison for boundary 1, 2, 3 , and 4, which have 5, 20, and 500 sources for the anticline model, respectively. The reflections from four interfaces are seen in $0.33 \mathrm{~s}, 0.83 \mathrm{~s}, 1.11 \mathrm{~s}$, and $1.63 \mathrm{~s}$ for anticline model, 12 results in total. In these figures, the yellow arrow shows the reflection from the coal layer, and the red rectangular boxes show the reflections from interfaces.

According to the anticline model results, the zero-offset VSP method is more successful to solve the coal layer than the surface seismic method (the red rectangular box in (b) corresponding to second anticline structure by the yellow arrow around $0.81 \mathrm{~s}$ ). Generally, 
both methods are good to solve the 1st reflection together in (b) and in (c). The surface seismic method is successful to solve the 3rd reflection from (the red rectangular box around $1.11 \mathrm{~s}$ ) better than the zero-offset VSP method. Both methods, in general, have failed to solve the 4 th reflection.

Figures 71-82 show the retrieved surface seismic and combined with VSP the seismic seismogram, VSP seismogram, and synthetic seismogram comparison for boundary 1, 2, 3 , and 4 , which has 5,20 , and 500 sources for the anticline-syncline model, respectively. The reflections from four interfaces are seen in $0.2 \mathrm{~s}, 0.65 \mathrm{~s}, 0.81 \mathrm{~s}$, and $1.91 \mathrm{~s}$ for anticline model, 12 results in total. In these figures, the yellow arrow shows the reflection from the coal layer, and the red rectangular boxes show the reflections from interfaces.

As for the most complex model, anticline-syncline, sometimes the surface seismic method is successful to solve the 3rd reflection from (the red rectangular box around $0.81 \mathrm{~s}$ ) better than the zero-offset VSP method, sometimes the zero-offset VSP method is better than the surface seismic method. When we look at the Figures, in general, the VSP has been unsuccessful to detect the reflections. The surface seismic method solves the 1st reflection better than the zero-offset VSP method. Also, both methods have been unsuccessful to detect the reflection from the 4 th interface. 


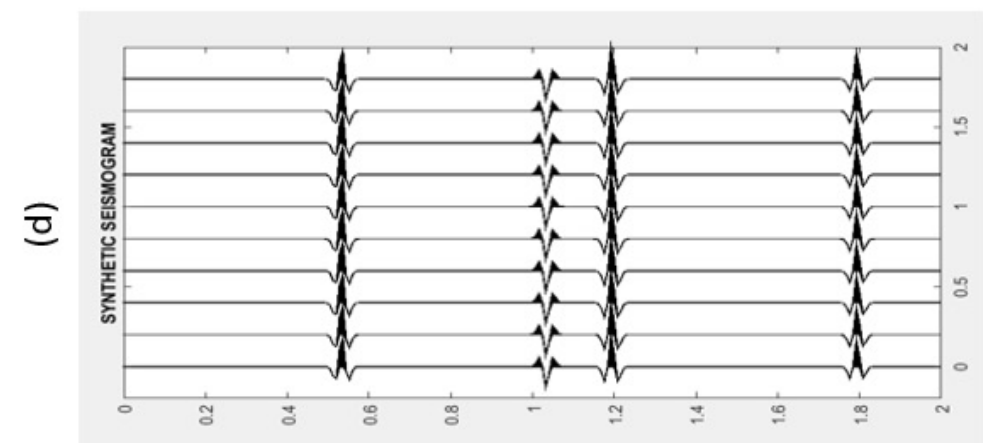

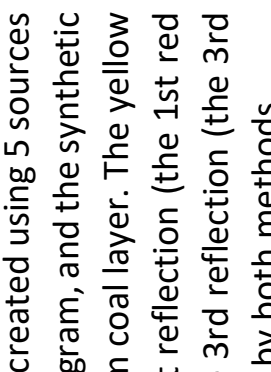

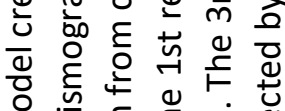

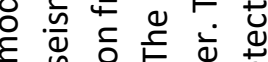

넝
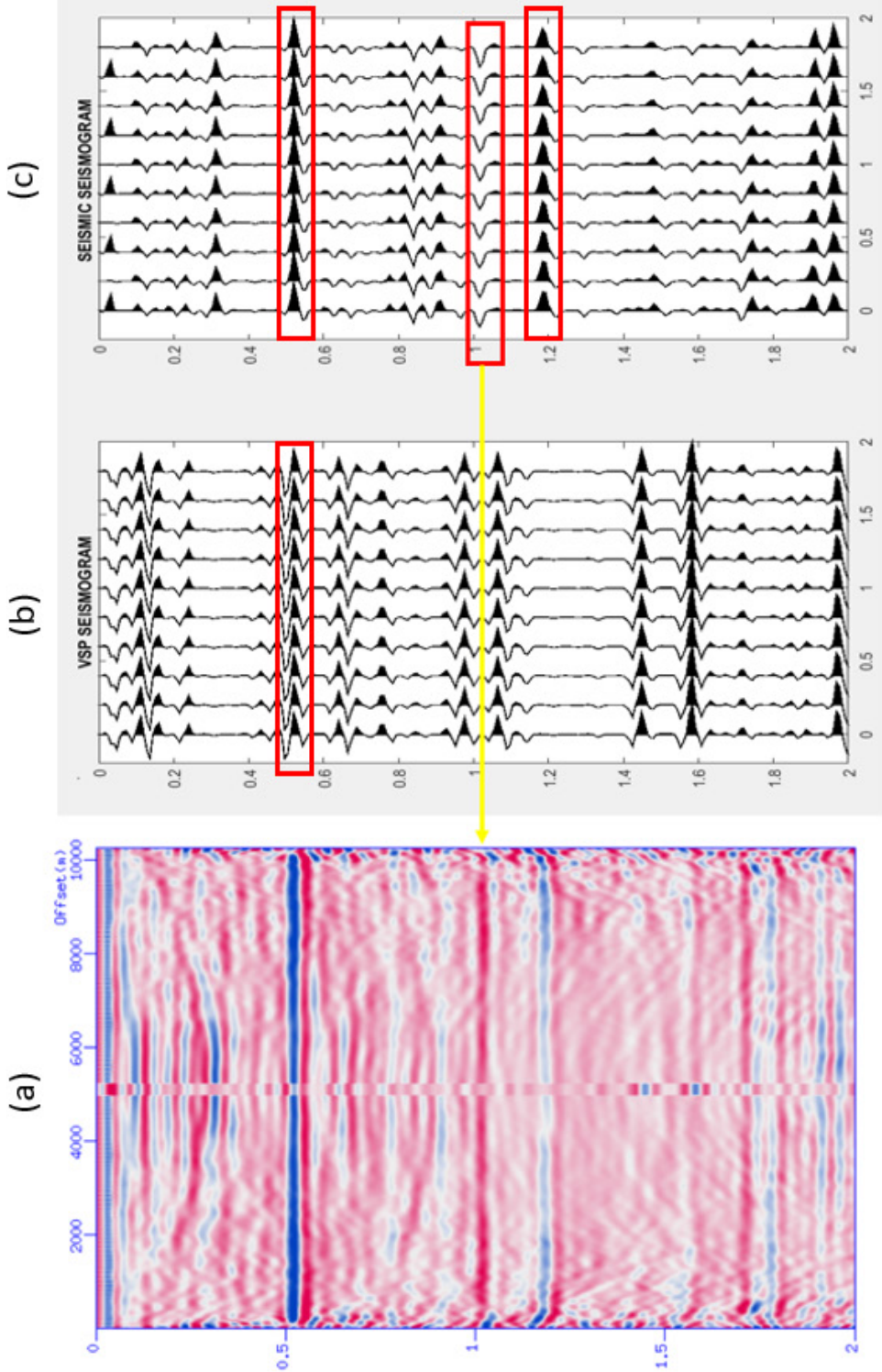

药

क व 0

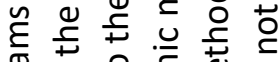

एँ

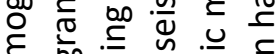

员

U $\frac{n}{d}$ है

थ थ 仓 원

艺

जิ

듄

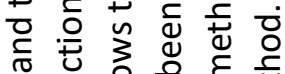

त

जิ ज ज

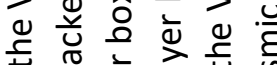

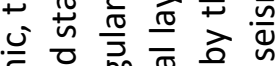

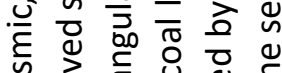

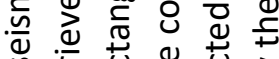

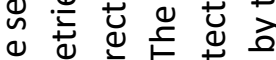

џ ब

Чั

ธิ

永

으웡 巳

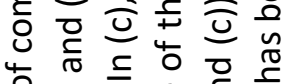

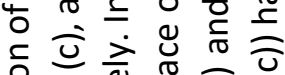

응

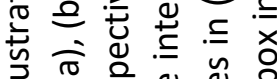

ब

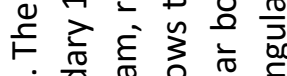

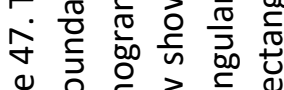

ข

话市 


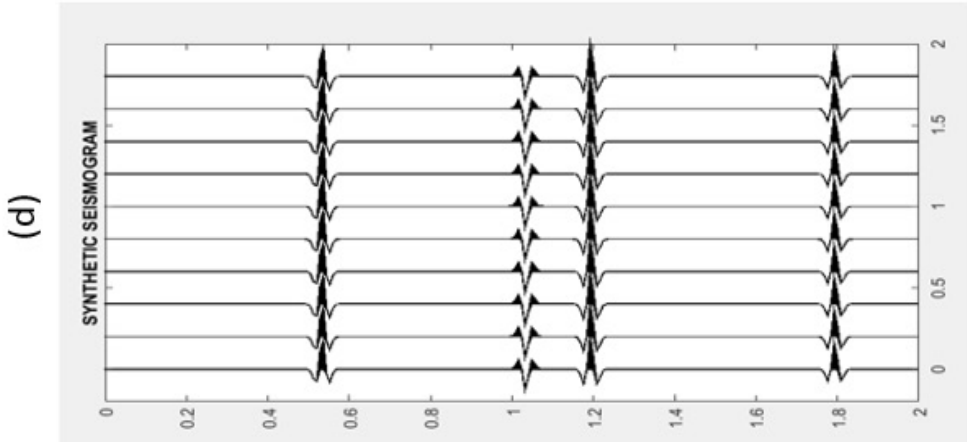

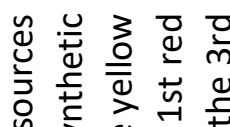

号至

10

言 它

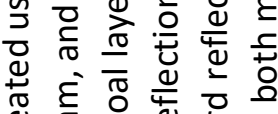

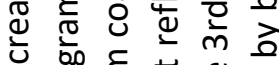

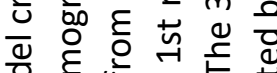

ठํ

ह

䒕 $\frac{\cup}{\varepsilon}$ 导
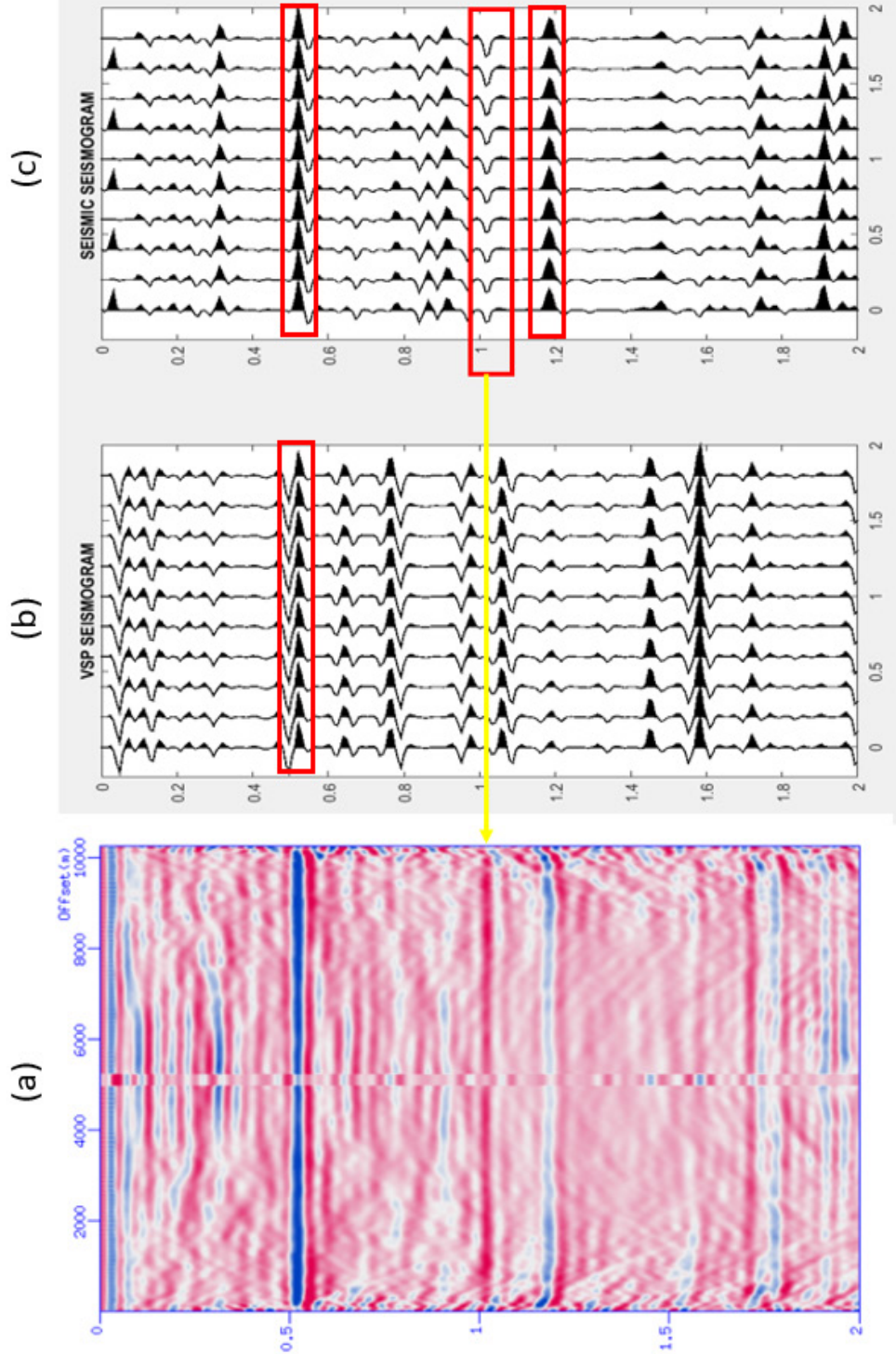

๘

ํํำ

ரัँ

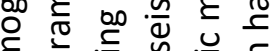

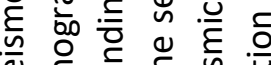

ज ह

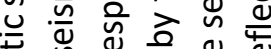

थ

艺 令

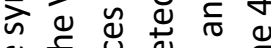

¿

† ᄃ்

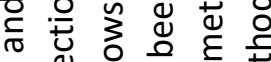

जं

๗

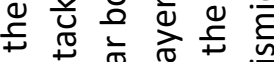

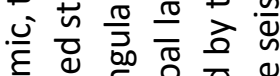

产

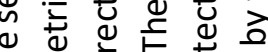

$\stackrel{\circ}{+}$

Чั

ธ

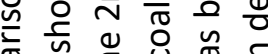

帝

Uั口

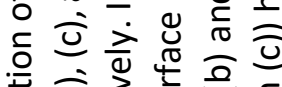

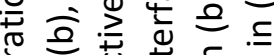

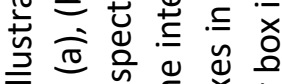

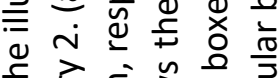

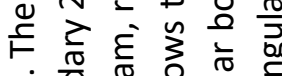

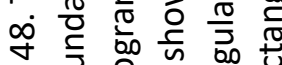

ฯ 일

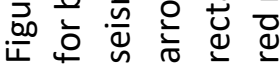



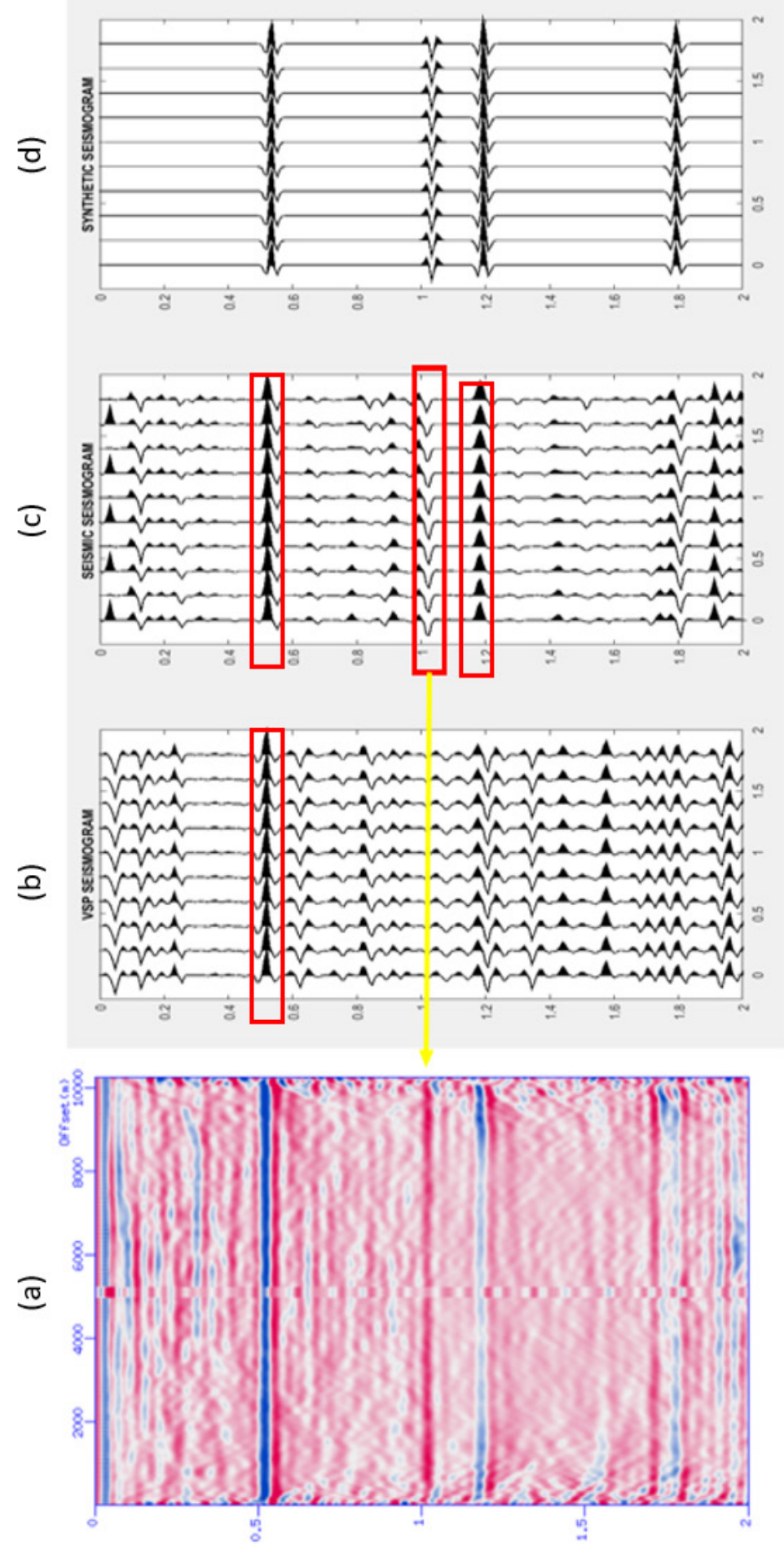

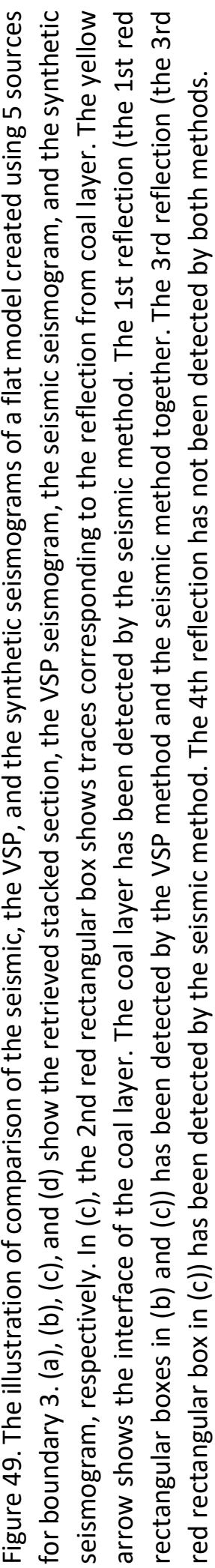




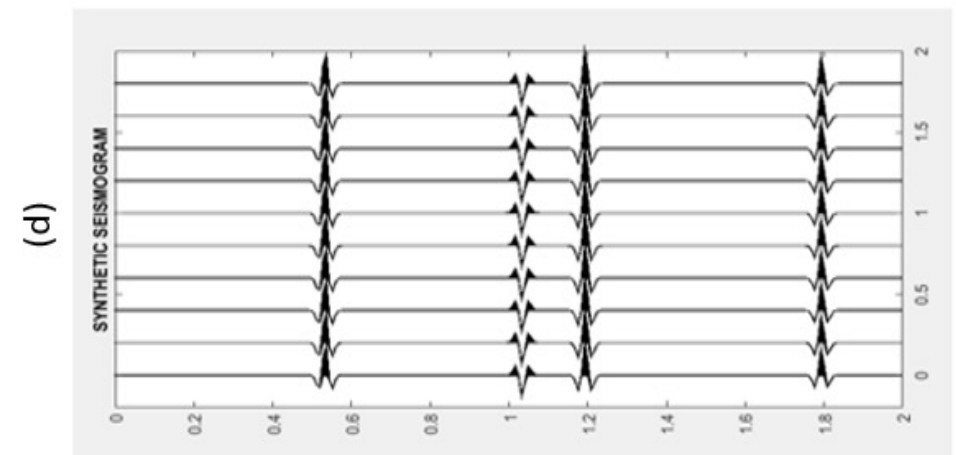

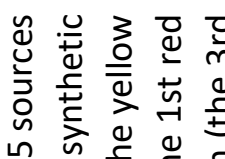

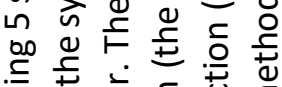

ড ড

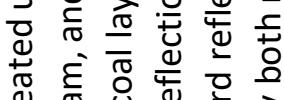

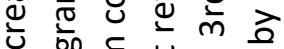

守

동

出
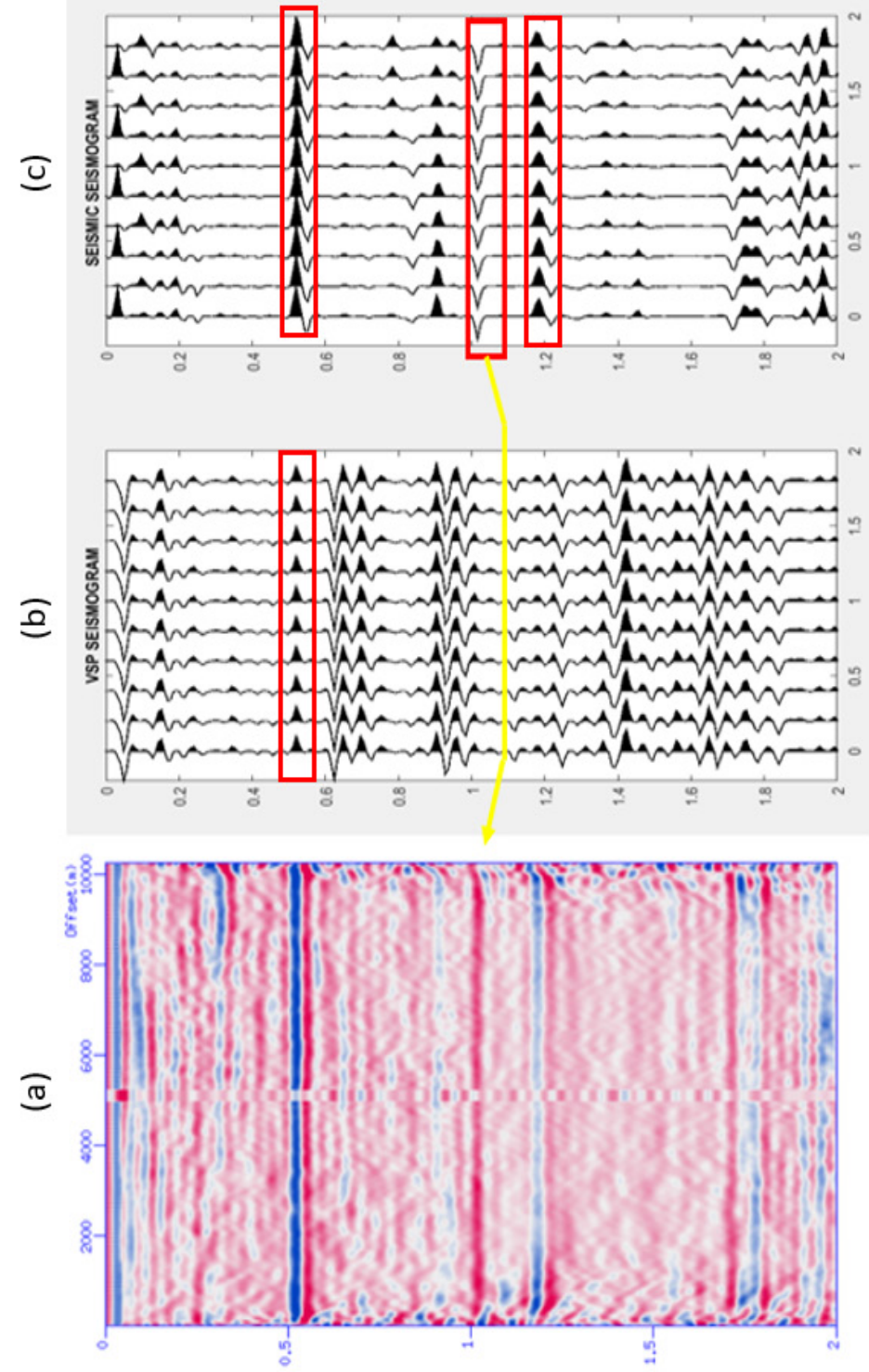

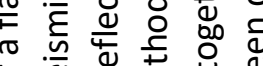

पั

ยิ

ฮั

ग0

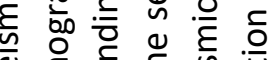

凹

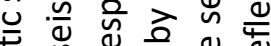

थ

華 令

जे

凹

†

ᄃ

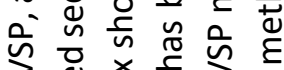

๗

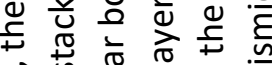

u

ह

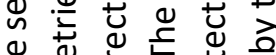

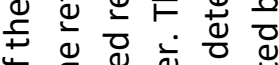

廿ั

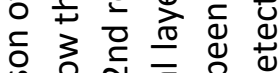

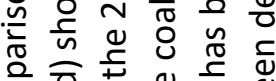

을워 웡

ชั은

U

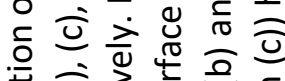

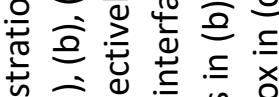

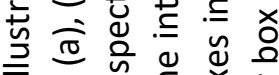

ฆ

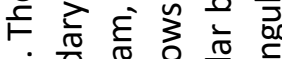

กำ

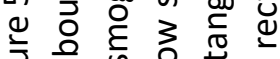

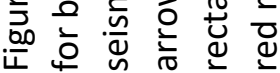




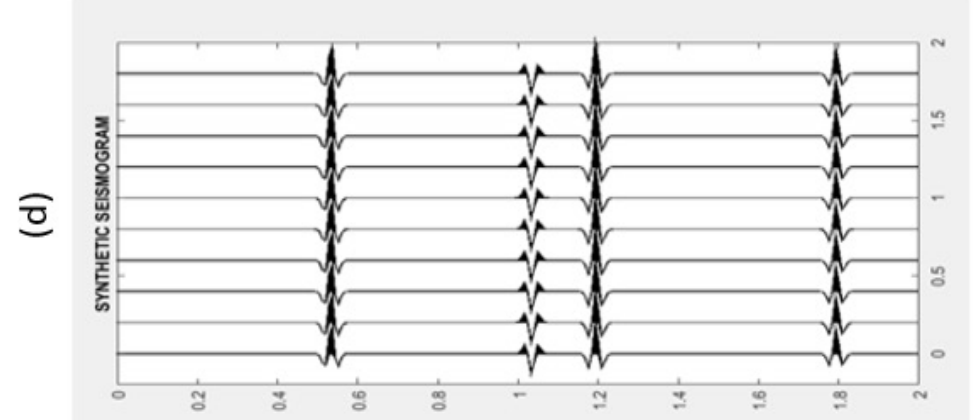

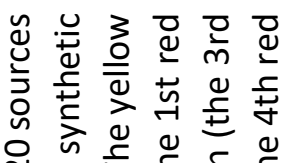

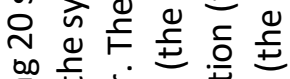

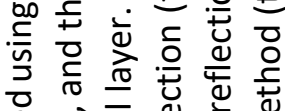

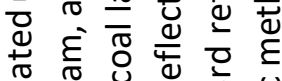

过

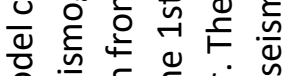

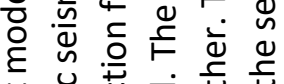

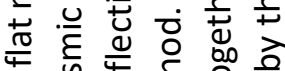
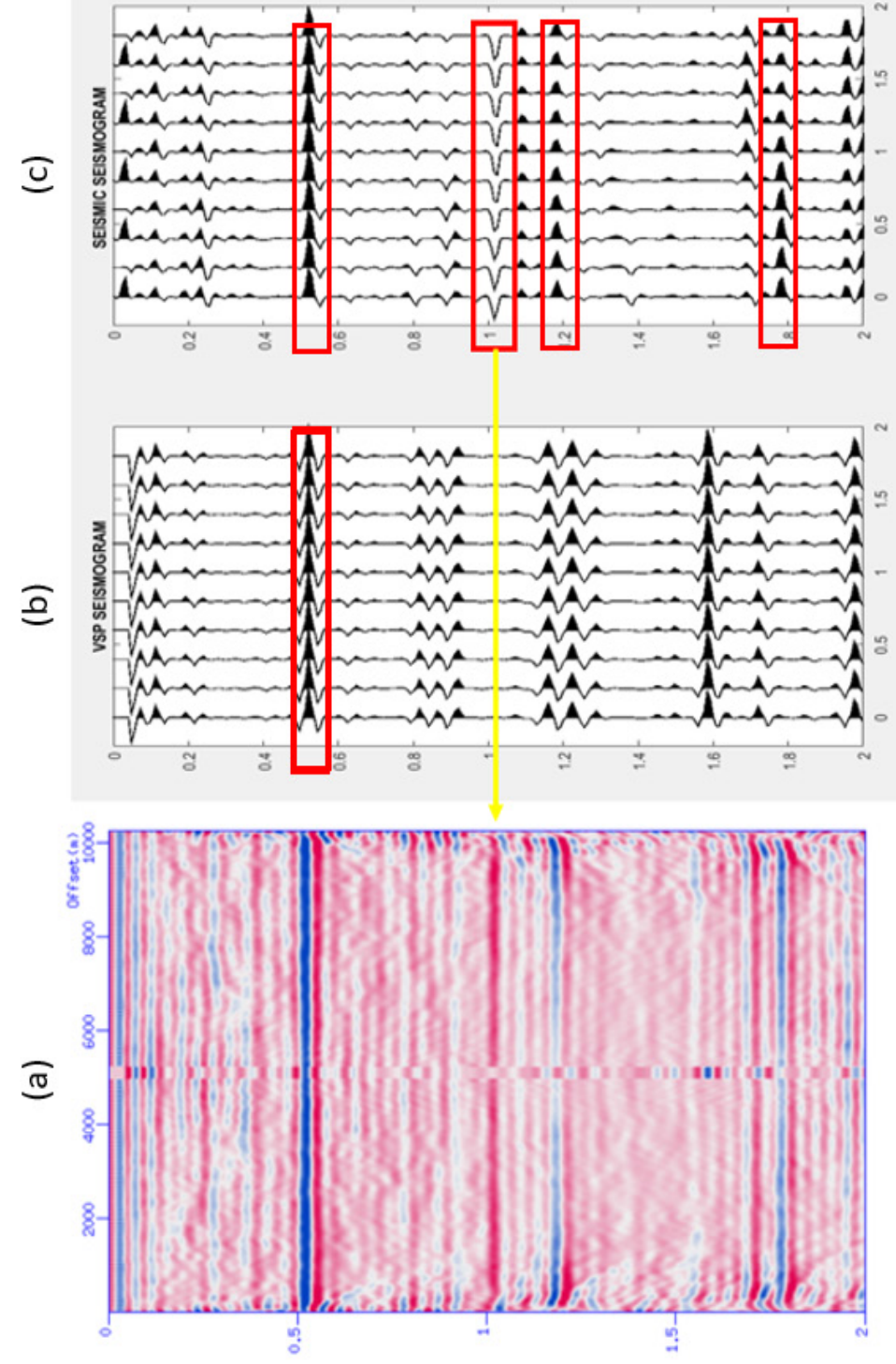

ப थ

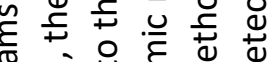

तั है +

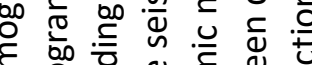

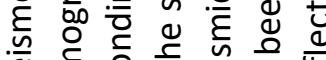

ज $\frac{\varepsilon}{n}$ ठ

.

苞 心

号

जे

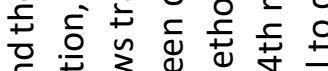

它

ก

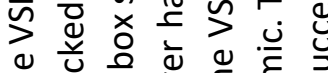

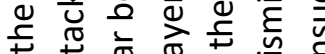

u 芯 $\frac{\pi}{5}$ 元

ह

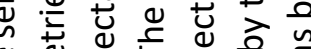

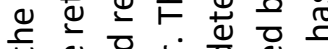

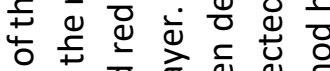

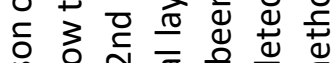

는 술

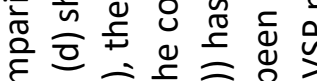

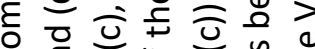

它

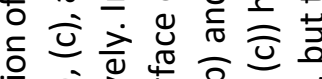

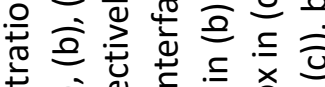

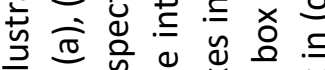

言

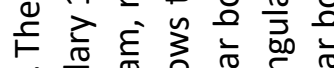

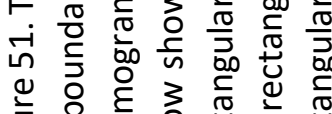

证 衣 

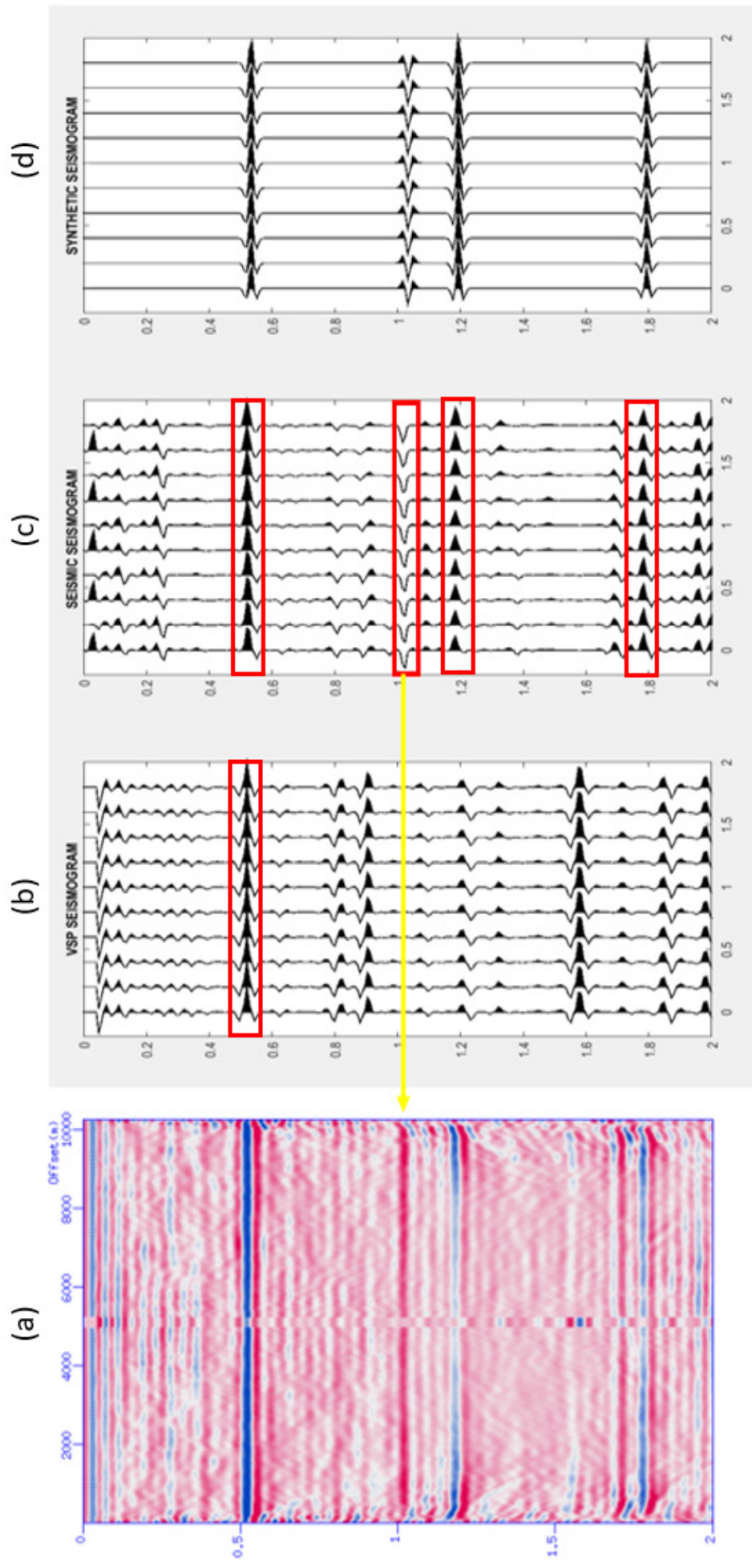

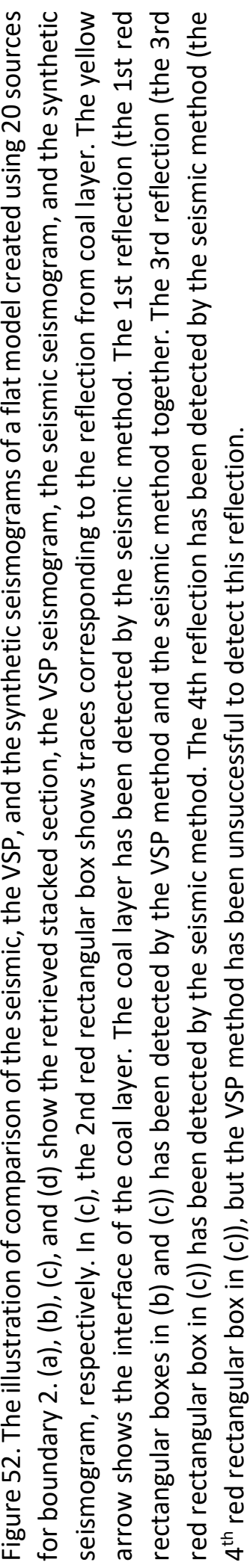



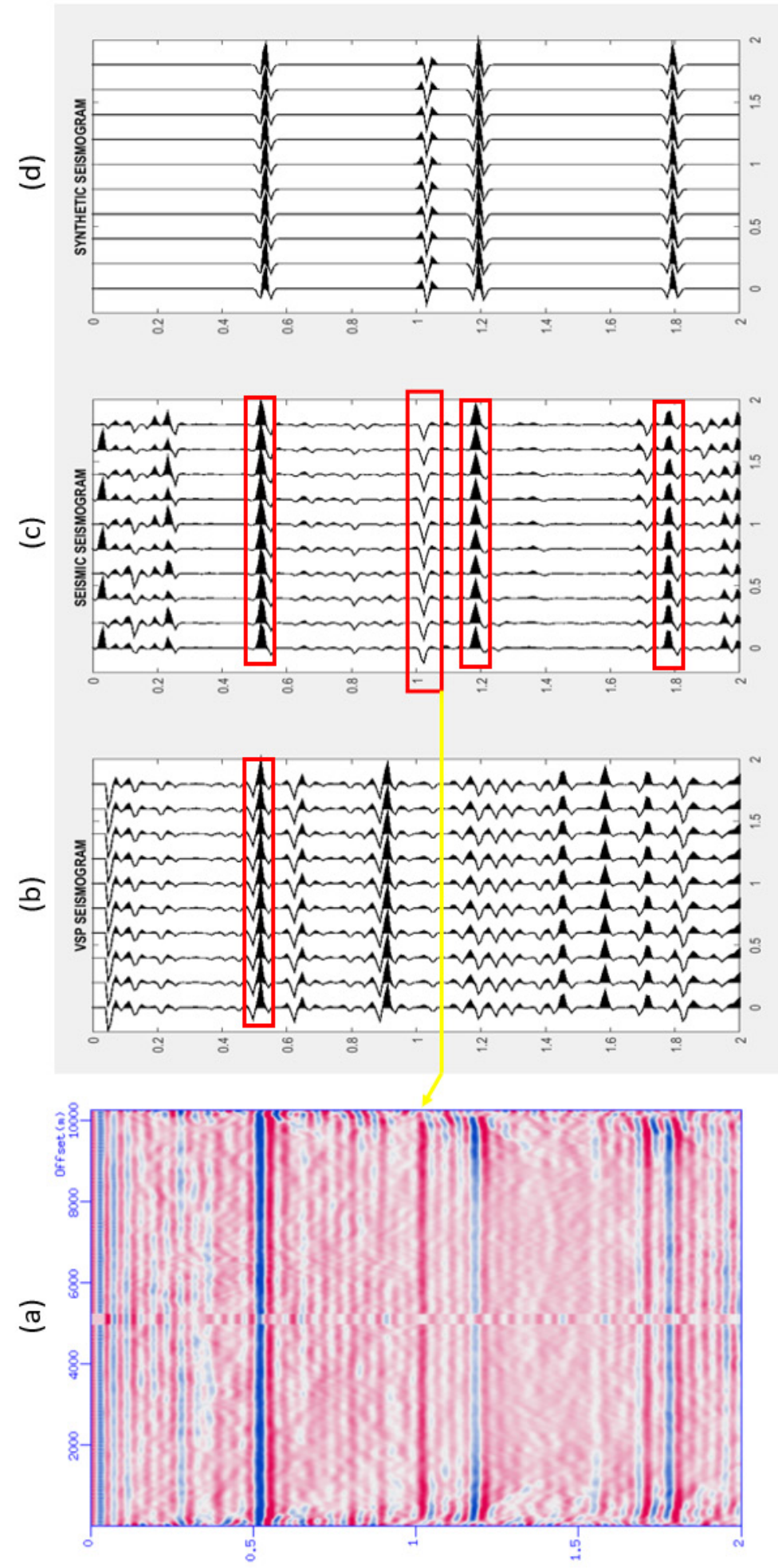

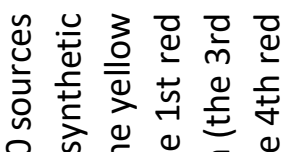

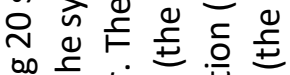

品 声

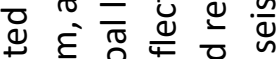

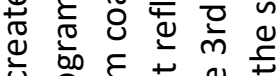

ป 잉

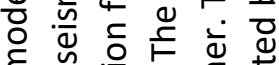

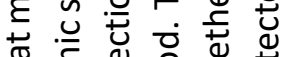

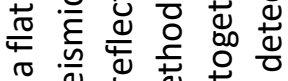

ᄂ

ยิ

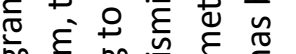

कू है

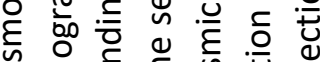

产

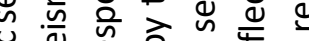

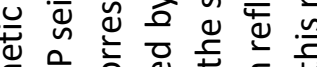

突 就

ऽ

के

\&

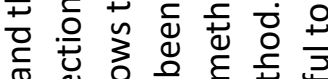

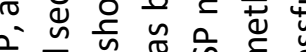

जे

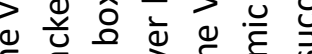

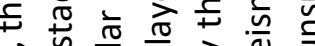

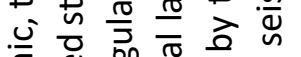

है

.

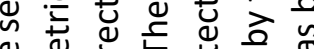

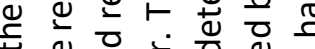

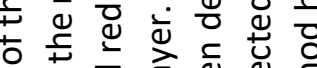

ว

눈

을 워월

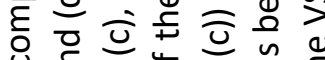

U.

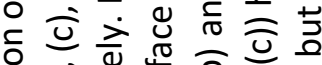

융

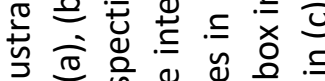

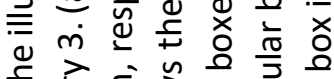

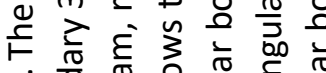

กี่

ข

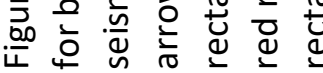



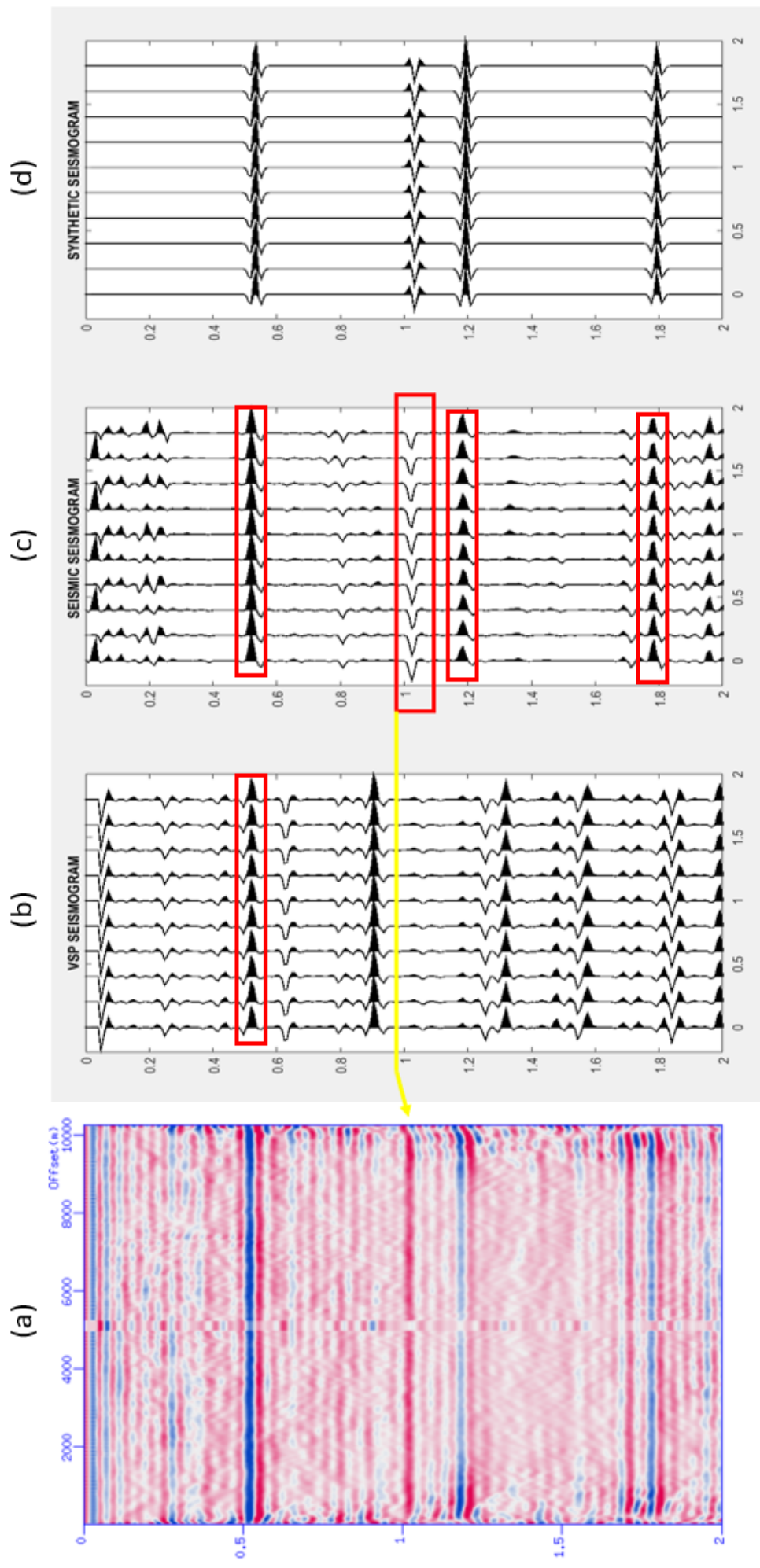

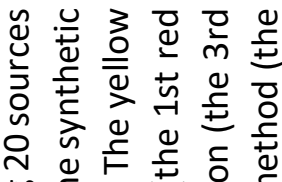

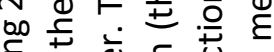

:

प्ष

凹

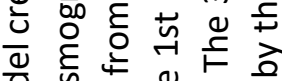

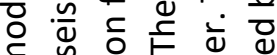

E

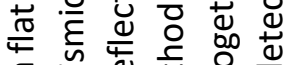

एँ

ڤै

要

它

嵌

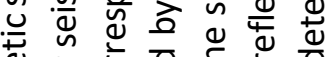

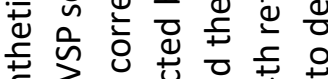

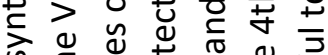

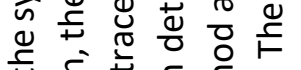

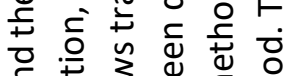

吕

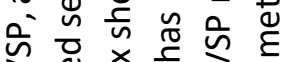

$>$ बै

峁

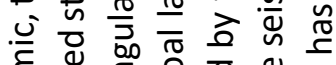

है

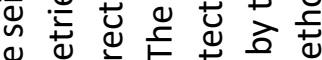

巳

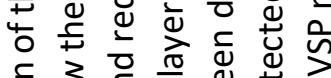

군

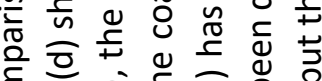

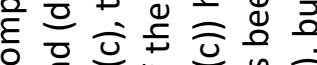

U.

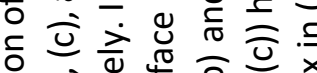

.응

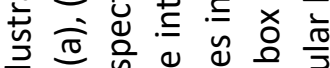

我焉

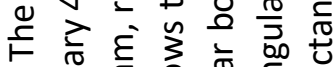

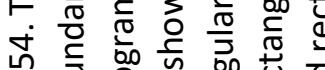

ஸी

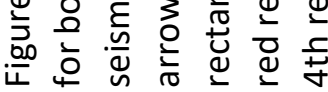




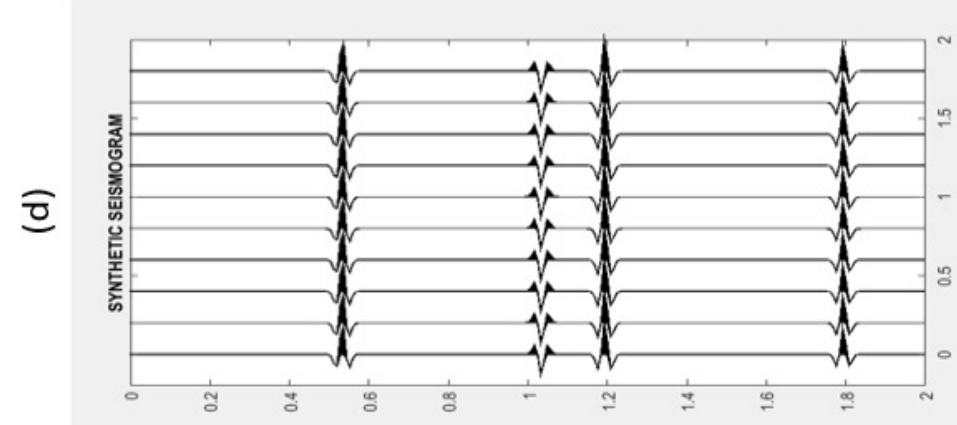

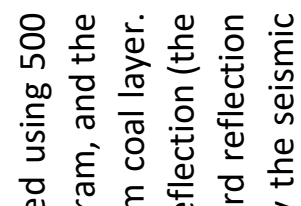

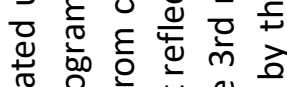

ญ

ब

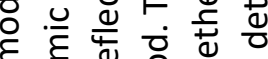

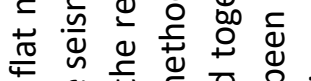

$\sigma$ ॠ

फั
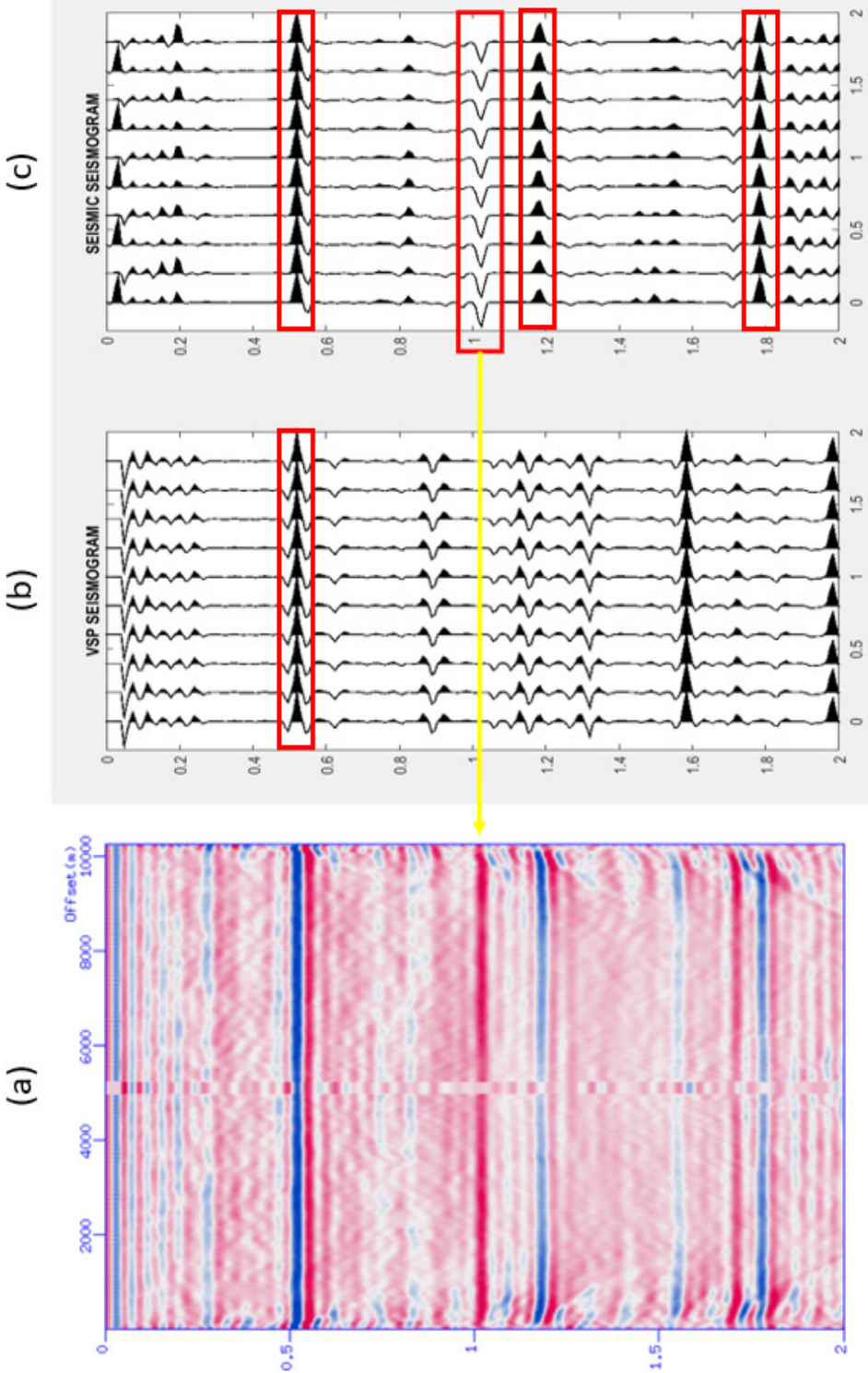

ज हो

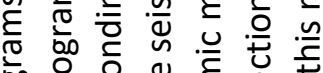

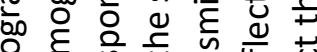

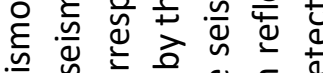

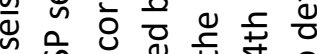

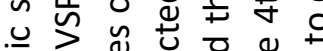

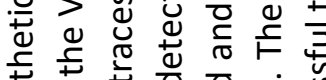

خ

जे

\pm ज

ত

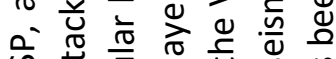

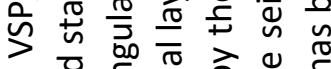

\& व

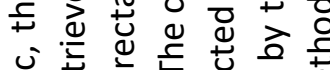

Uั

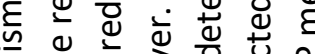

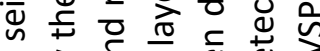

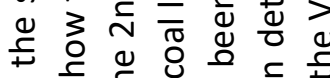

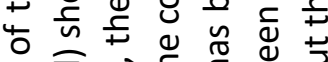

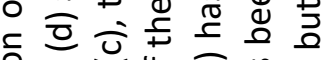

品

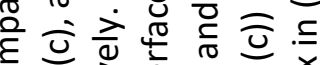

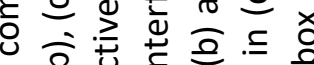

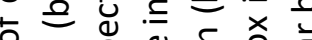

ᄃ

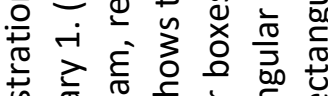

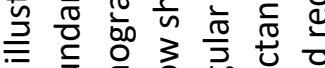

产

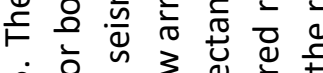

เं केष

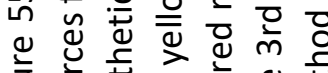

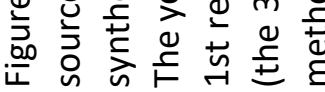



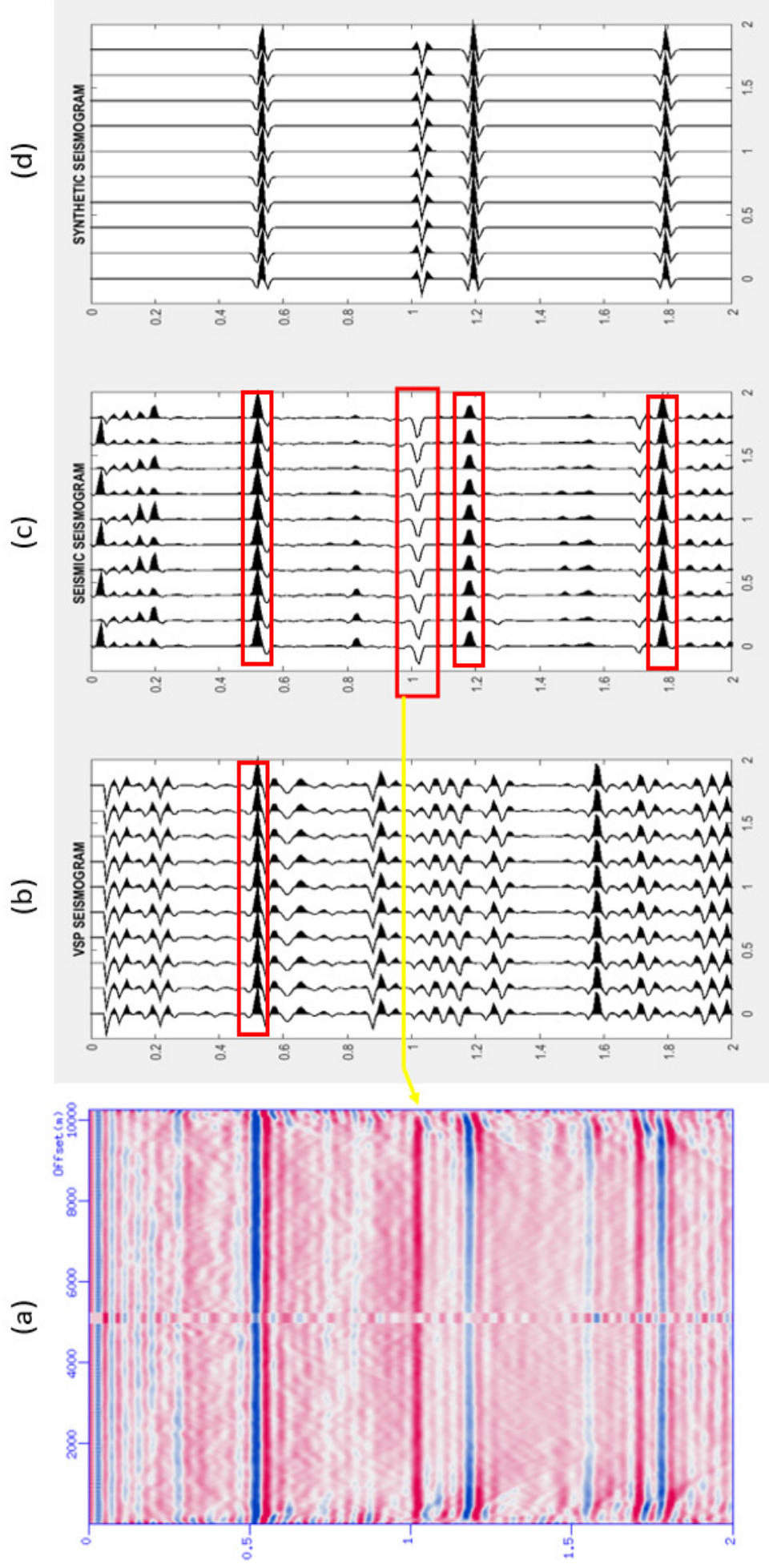

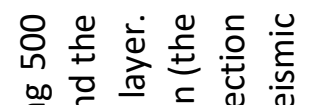

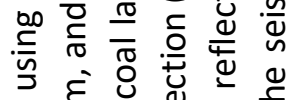

व

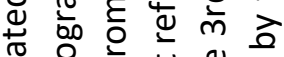

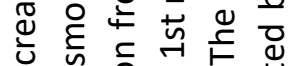

过

ठ .

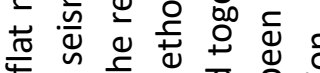

$\sigma$ 品

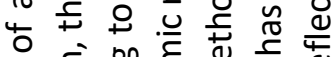

हो है हो

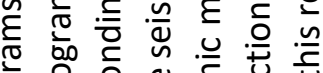

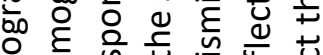

है

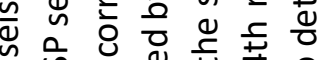

บ n

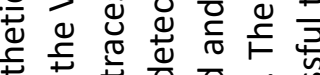

हं

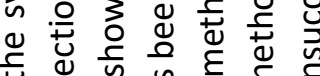

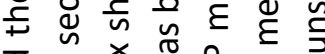

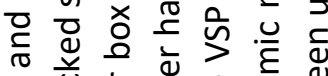

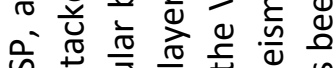

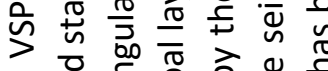

๑ ర

¿

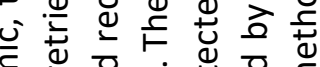

है

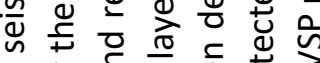

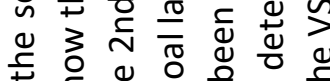

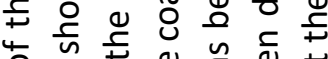

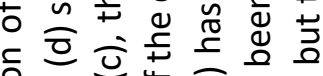

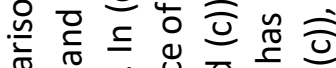

눈

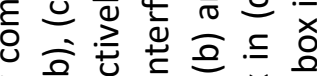

눙

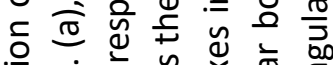

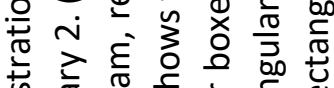

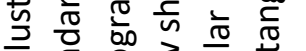

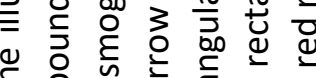

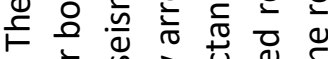

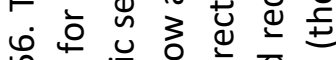

ᄂ

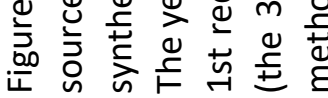



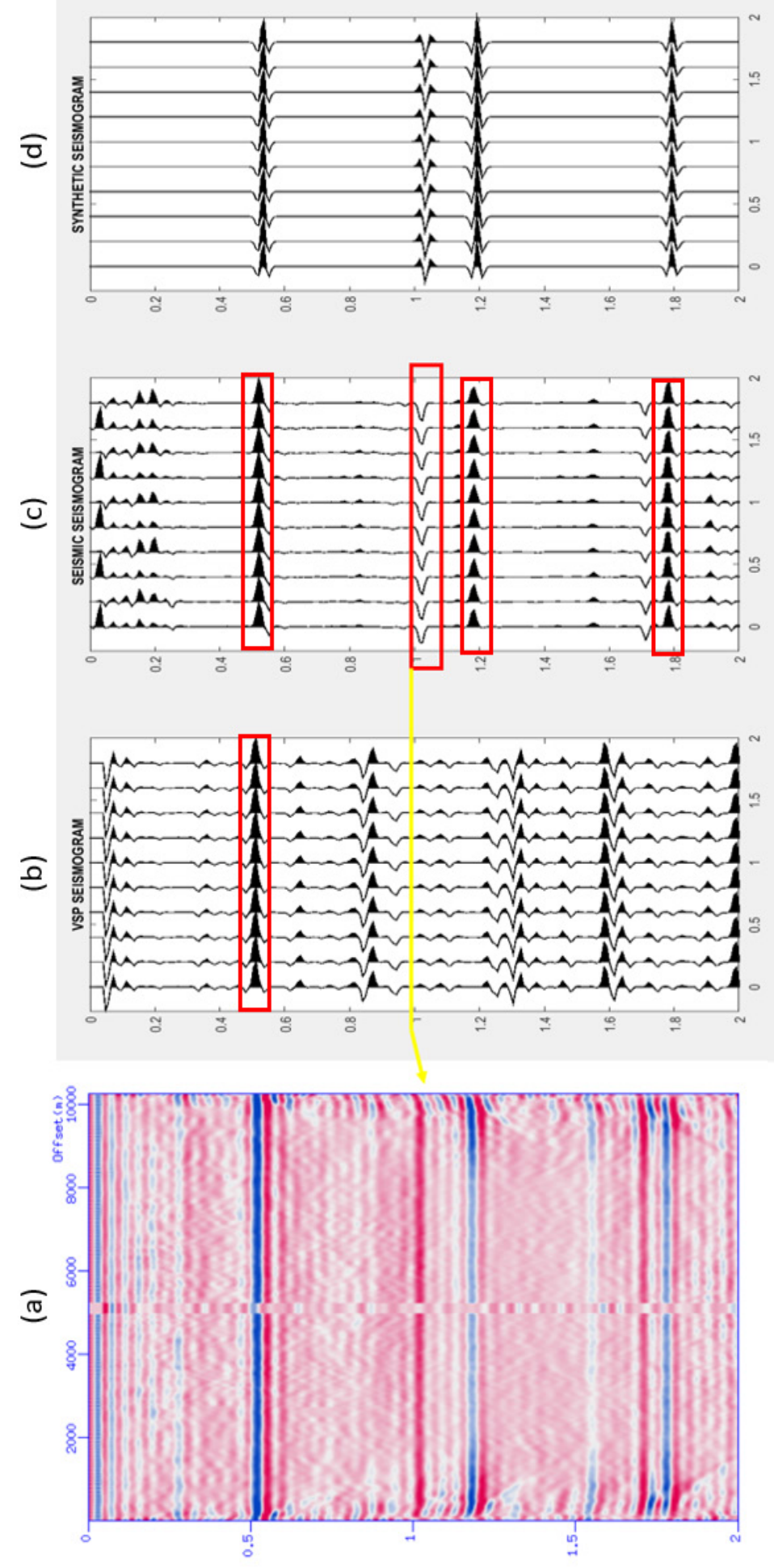

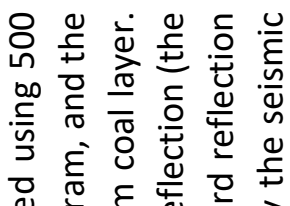

웡

ญ

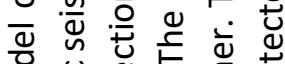

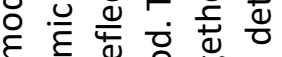

䒕

$\sigma$ \&

ᄂ

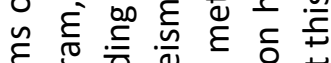

它

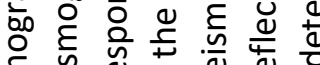

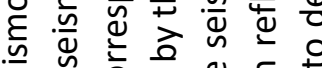

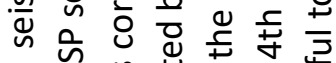

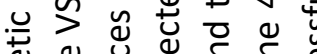

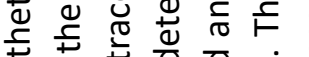

ปิ

जै

$+\infty x$ 出 $\varepsilon$ d

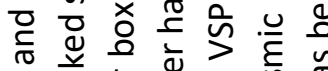

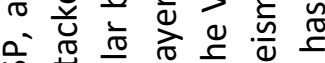

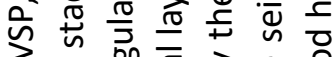

व

$\digamma$ य

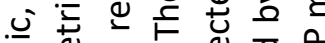

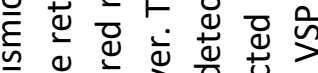

凹

¿

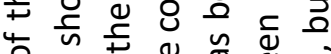

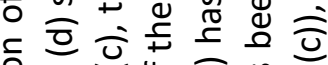

인

है

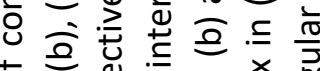

प。

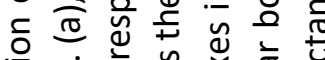

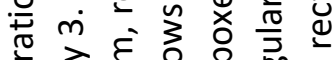

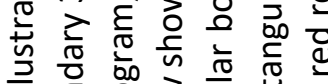

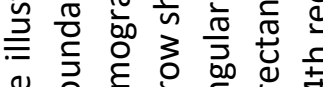

\&

ㄷํㄴ

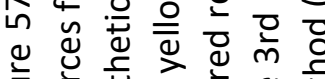

壳 

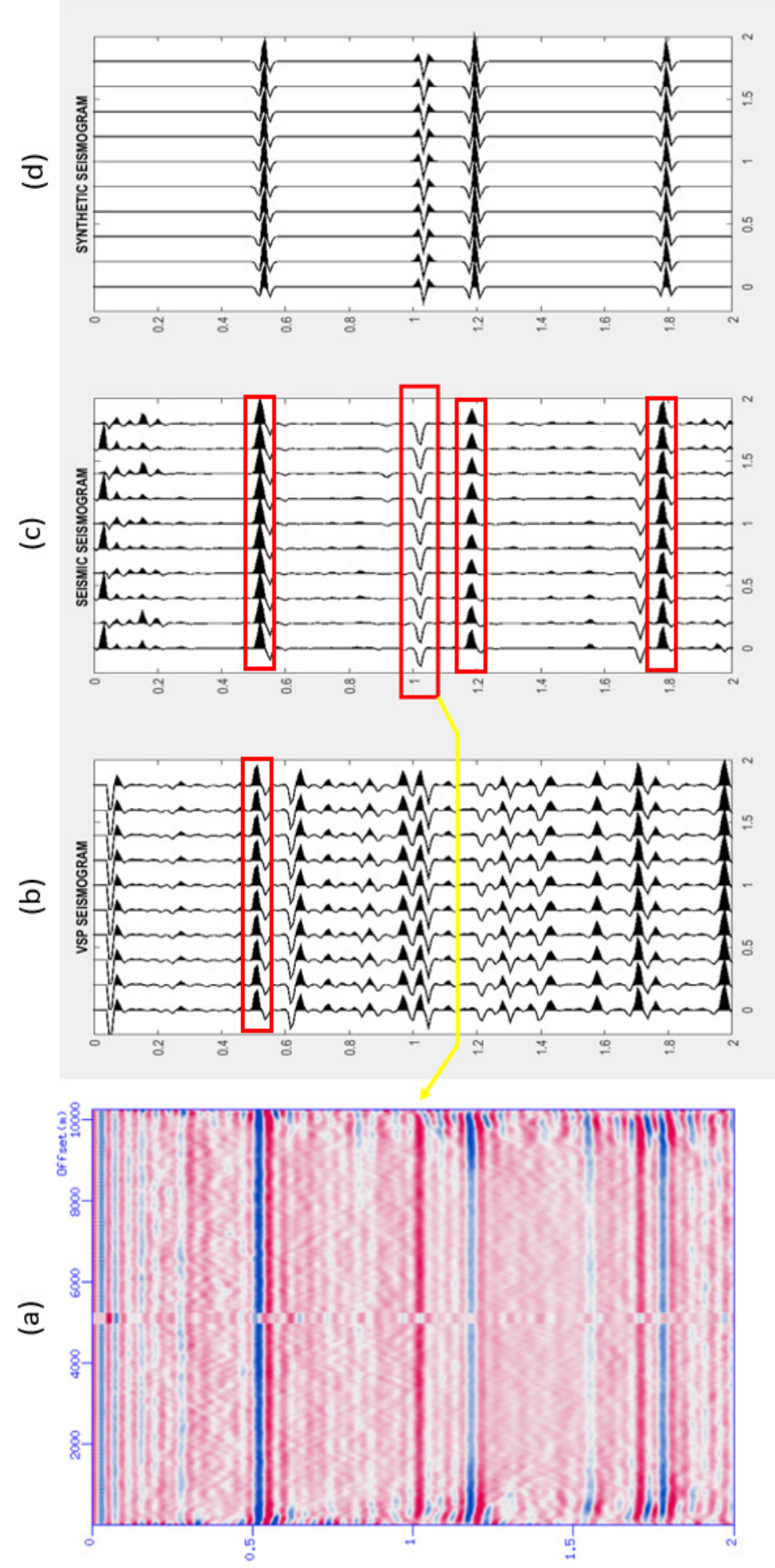

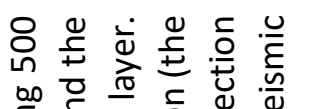

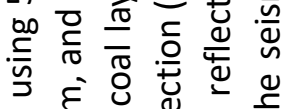

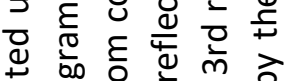

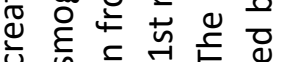

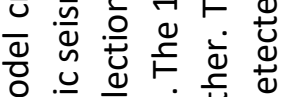

है

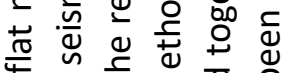

$\sigma$ 吼

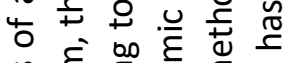

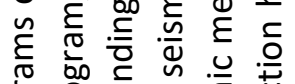

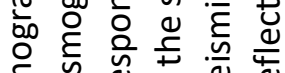

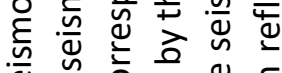

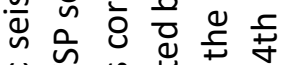

ปั

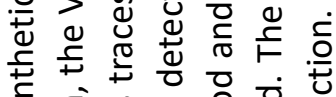

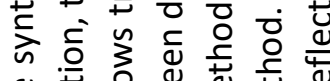

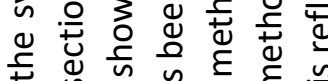

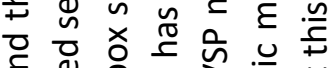

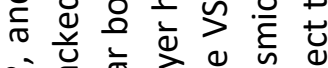

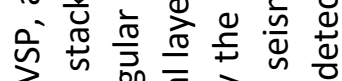

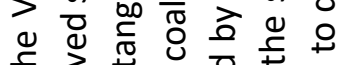

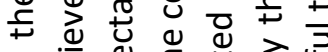

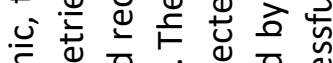

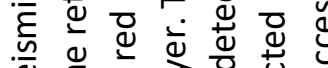

过

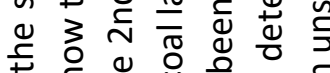

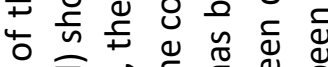

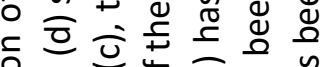

은 동

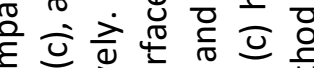

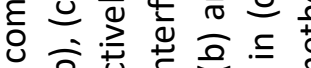

4 远政

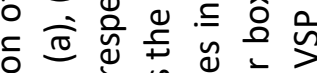

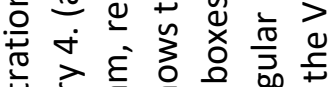

구의

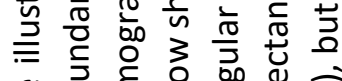

点

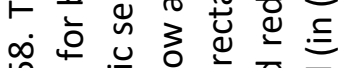

ก

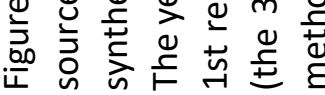



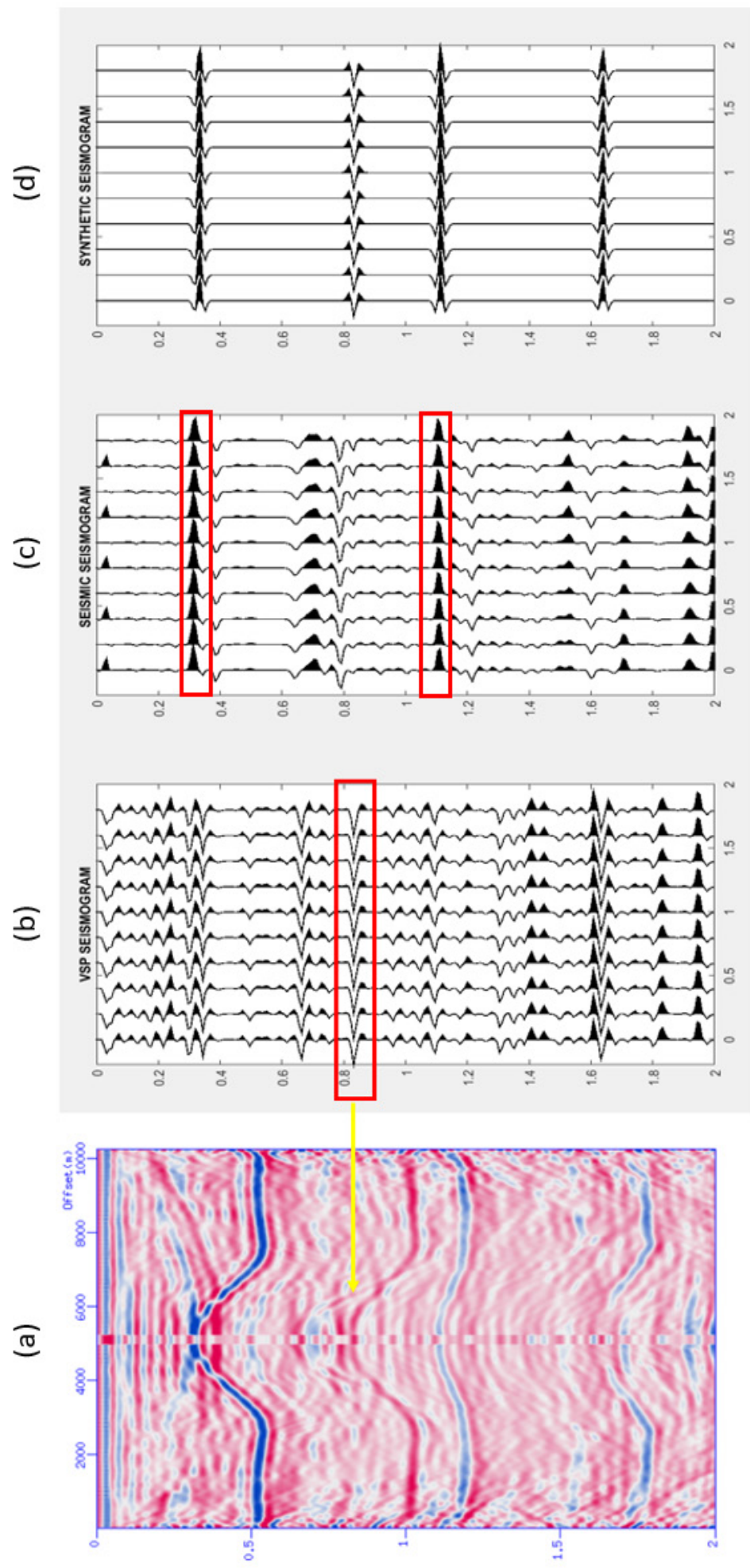

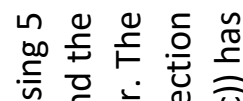

产 $\frac{0}{\sigma} \dot{\bar{\nu}} \frac{\mathrm{d}}{4}$

व

त

บั

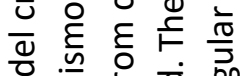

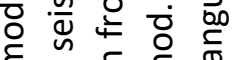

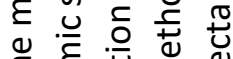

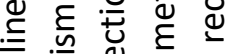

脑 $\frac{d}{4}$ 品

त \&

동

पँ है

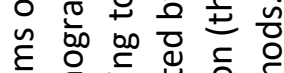

竞

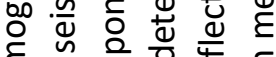

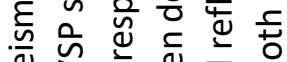

๗

는

む

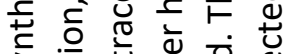

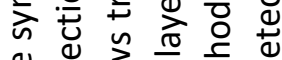

d

†过

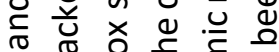

म

ज ठ

Ð む)

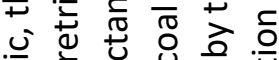

ह

$\frac{5}{4} \leftrightarrows \frac{\Phi}{5} \frac{Q}{4}$

ज 3 प

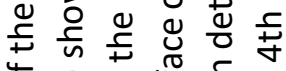

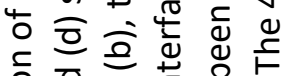

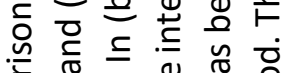

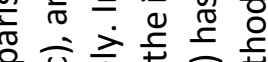

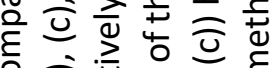

엉

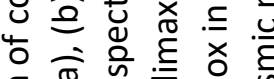

일

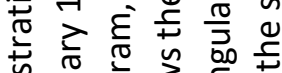

苟曹

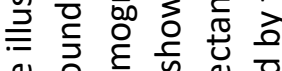

๘

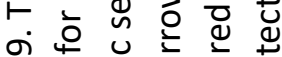

ก

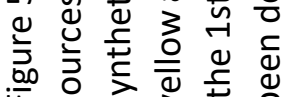



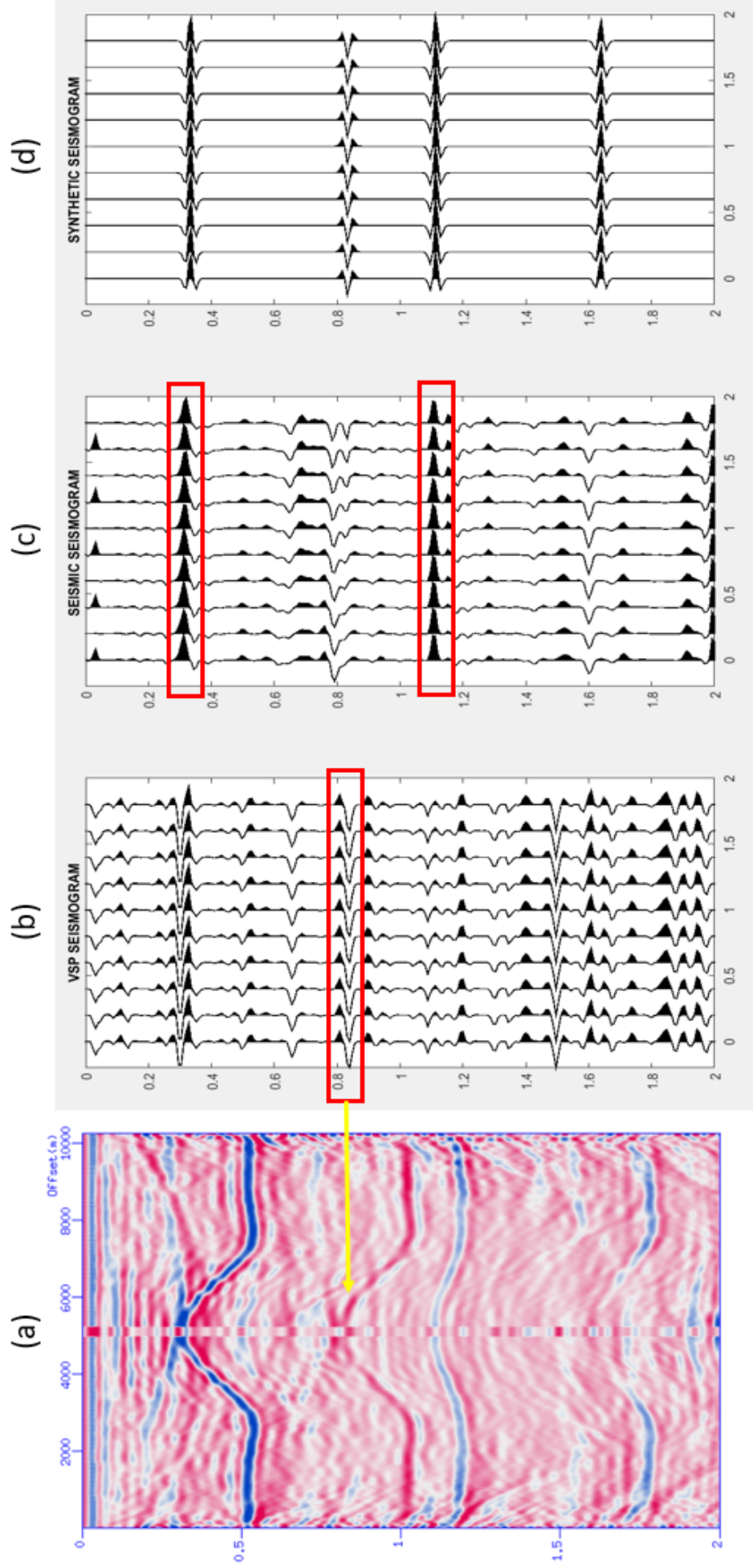

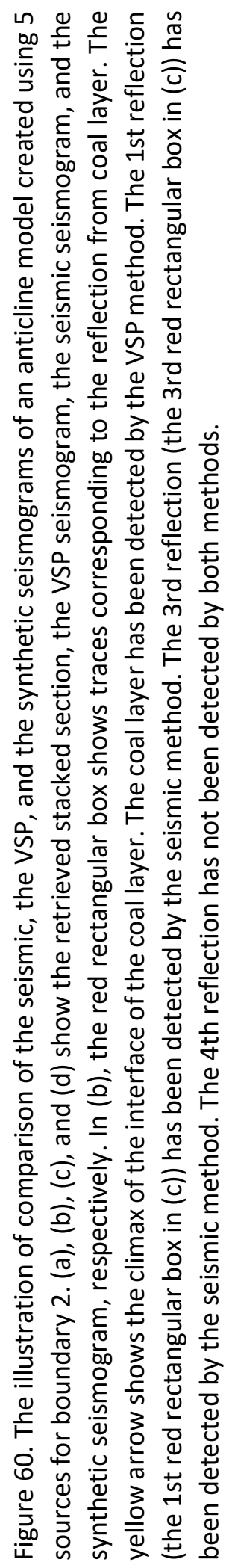



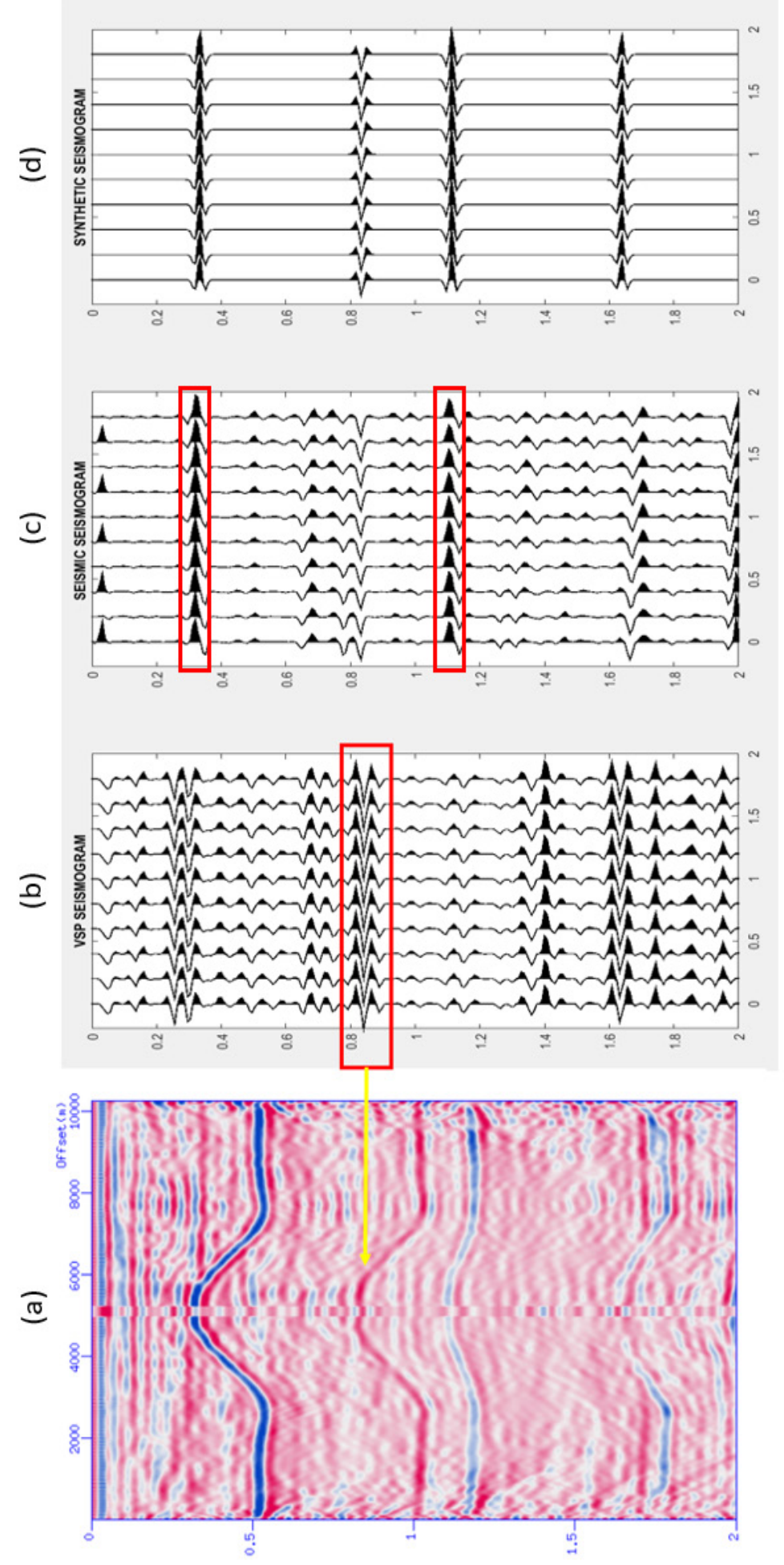

ᄂ

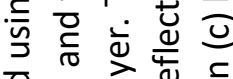

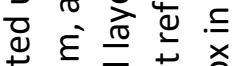

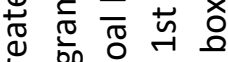

บั

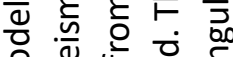

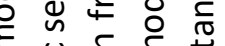

ब 을 음

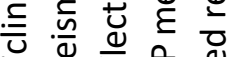

它 $\frac{d}{4}$ जे

त

曲

प实

है कू

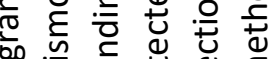

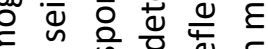

जิ ฏ 巳

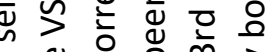

얼

¿ 仓

文 은

ज㐫

엉 उ

ত

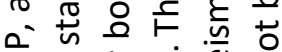

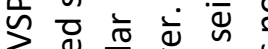

¿ d

厂 $\stackrel{0}{=}$

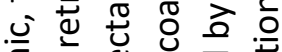

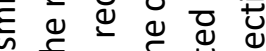

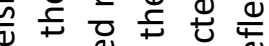

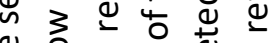

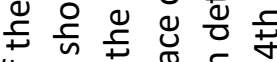

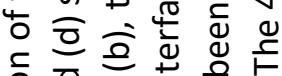

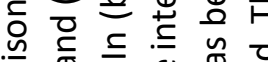

元

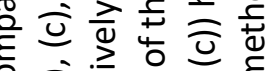

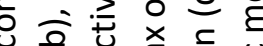

पㄷำ

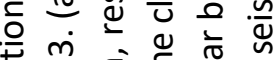

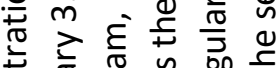

ज行

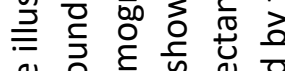

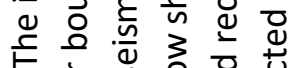

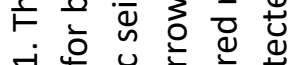

ठं

0

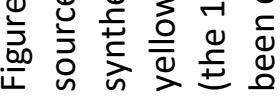




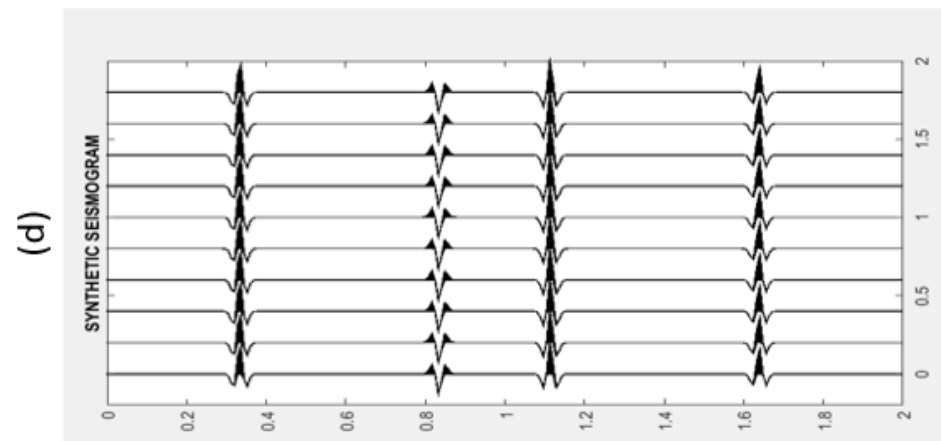

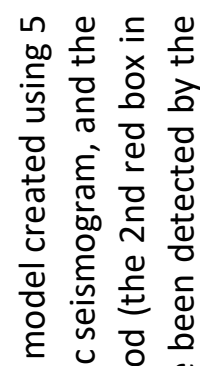

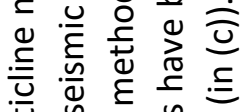

贲
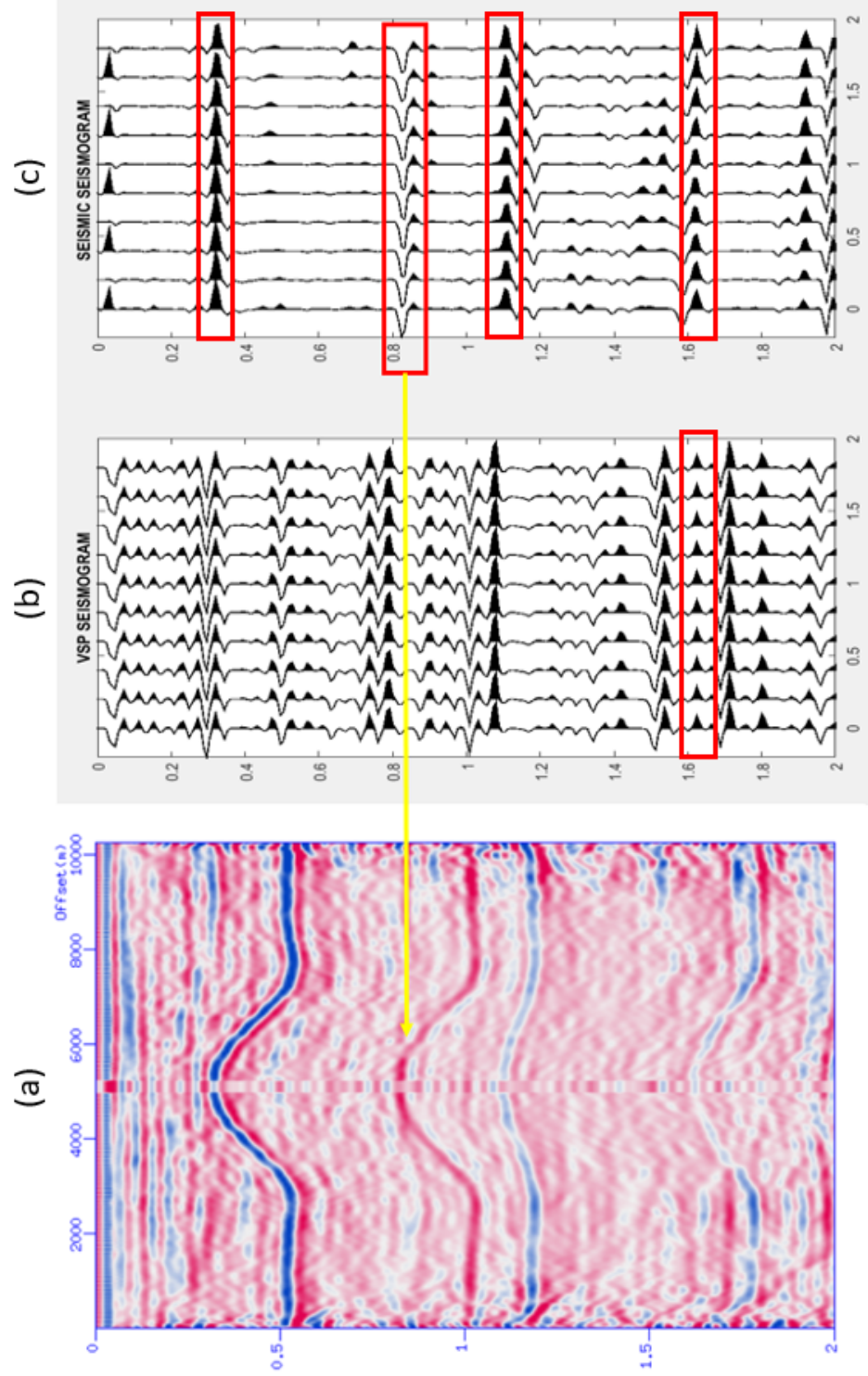

त ह प्य

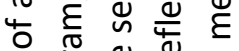

气

है

员

है जे ث્屯

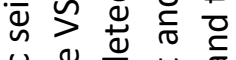

음

的䙳

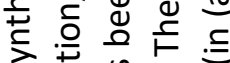

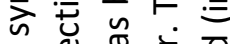

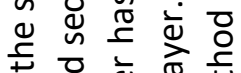

产竞离

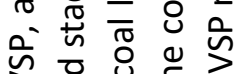

जั

๑ \.

ডي

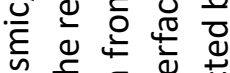

$\frac{\mathrm{M}}{\mathrm{N}}$ 古

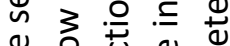

过 号

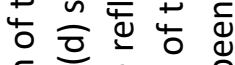

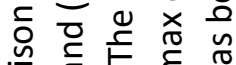

次

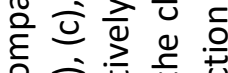

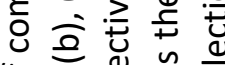

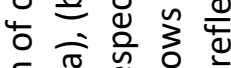

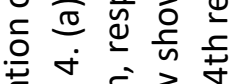

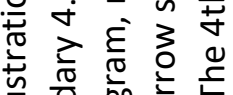

气 흥

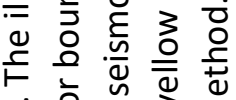

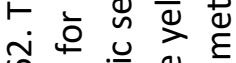

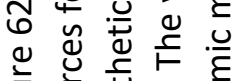

证 


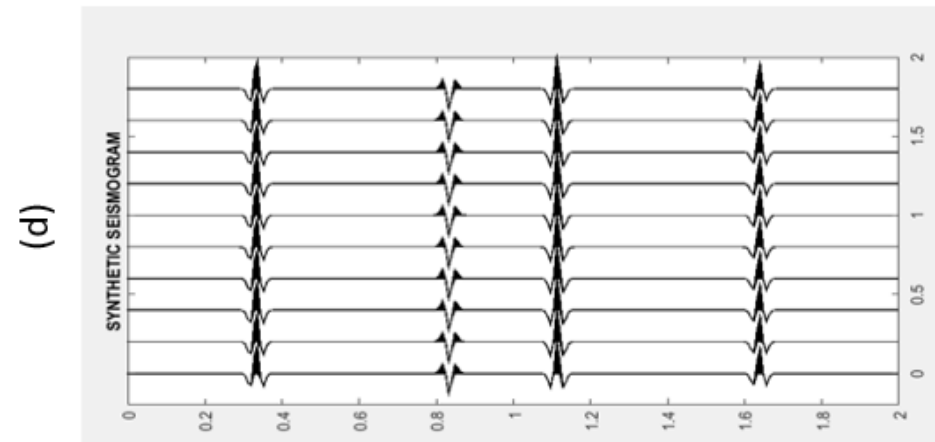

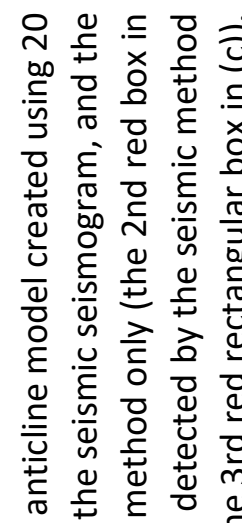
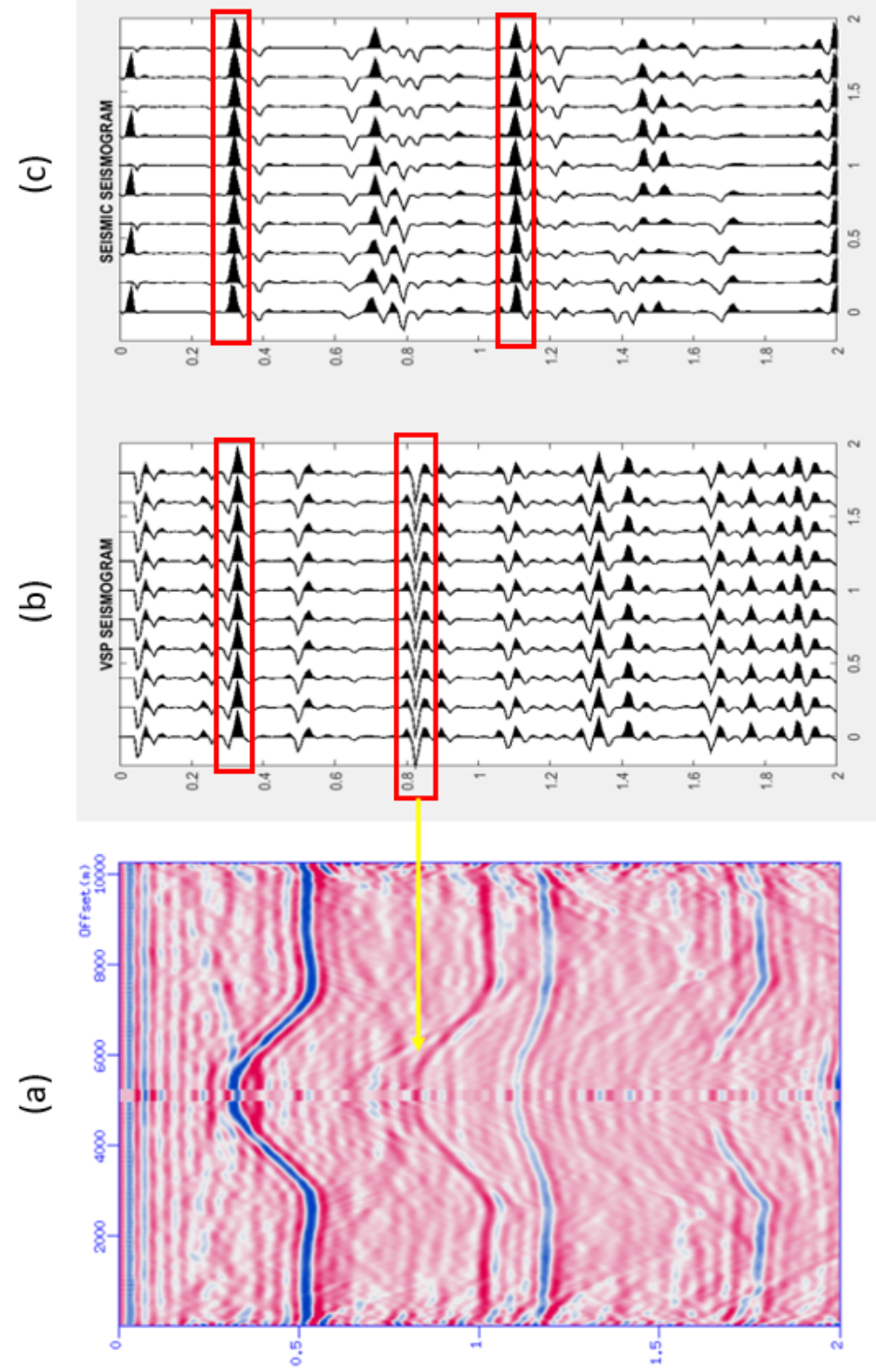

ते हे जे

प๐

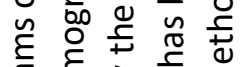

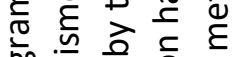

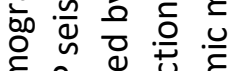

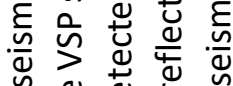

U⿺辶寸

过 d

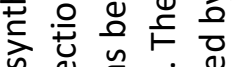

जे

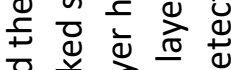

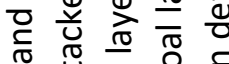

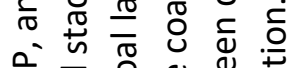

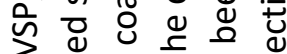

む)

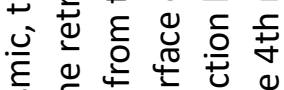

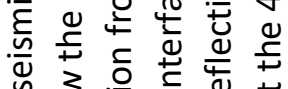

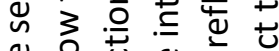

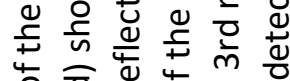

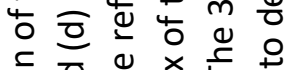

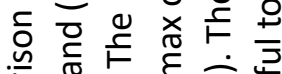

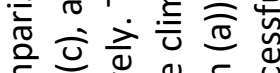

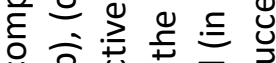

प्र

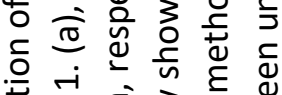

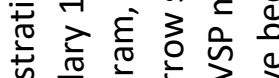

䒿

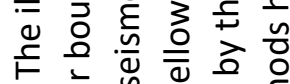

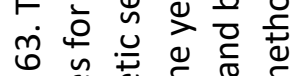

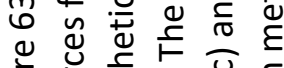

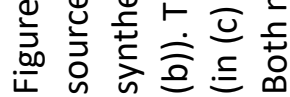



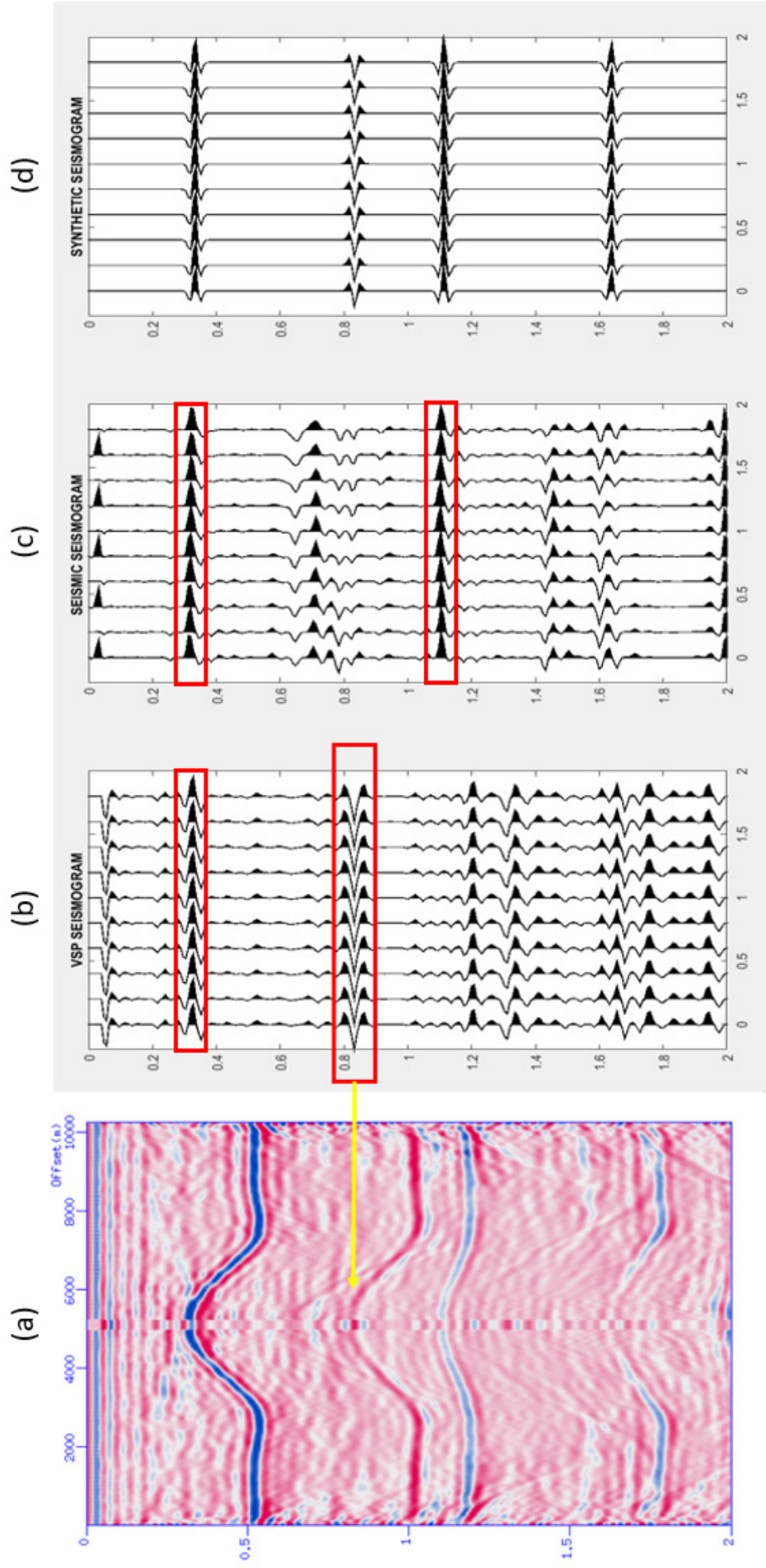

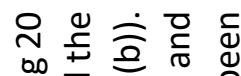

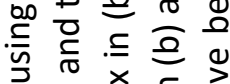

ปั

贾

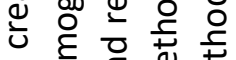

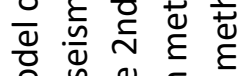

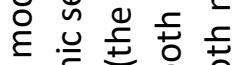

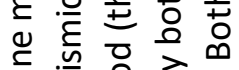

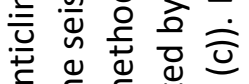

元

त हो जे 造

훙 $>\frac{8}{0}$

है

는

。ำ

है 造 究

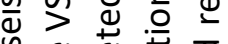

웡

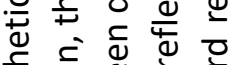

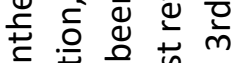

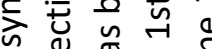

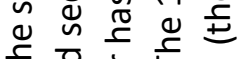

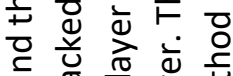

证

जे ठㅇ⿺

远

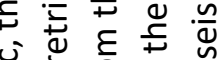

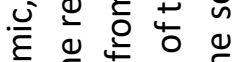

क

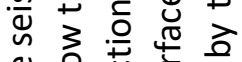

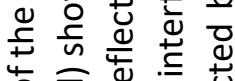

पे

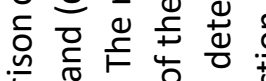

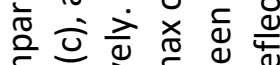

है

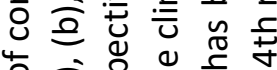

훙

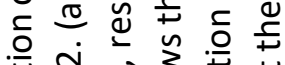

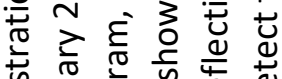

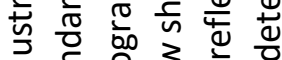

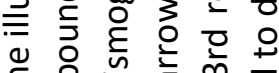

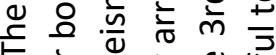

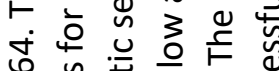

ठั

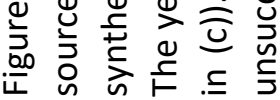




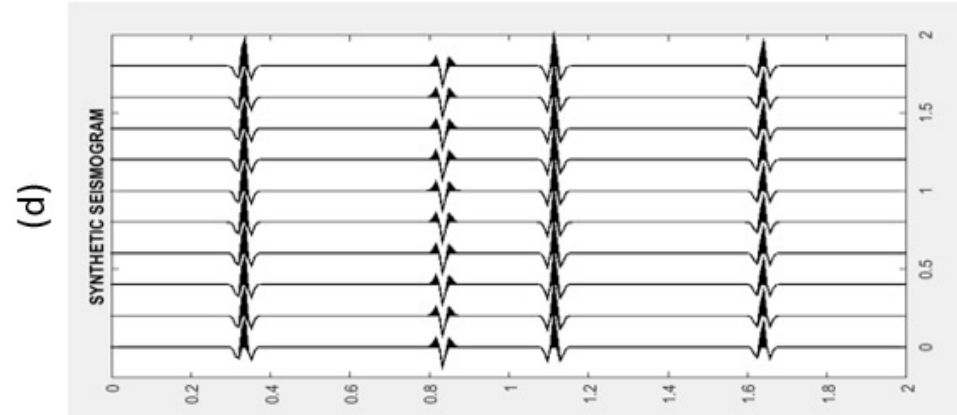

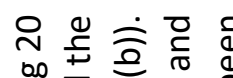

玄

ปั

苋

Чั山

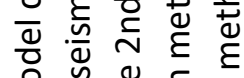

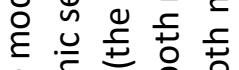

\& है 중 용

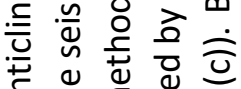
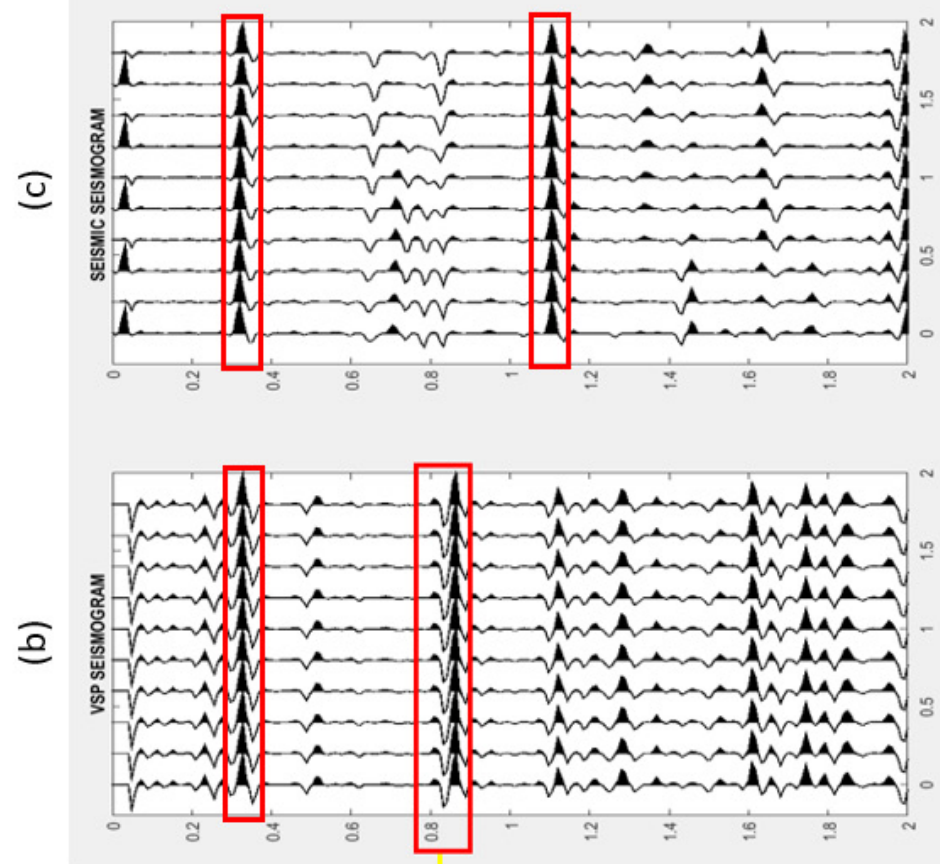

हो

पँ

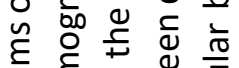

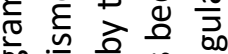

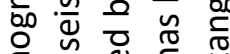

है जे ث્屯 :

$\Phi)$

U.

屯 ¿

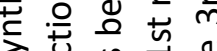

令焉先

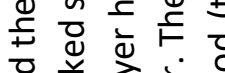

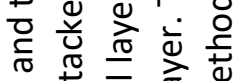

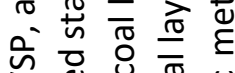

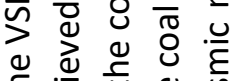

†.

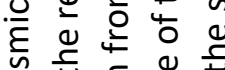

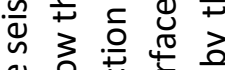

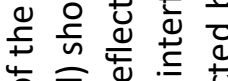

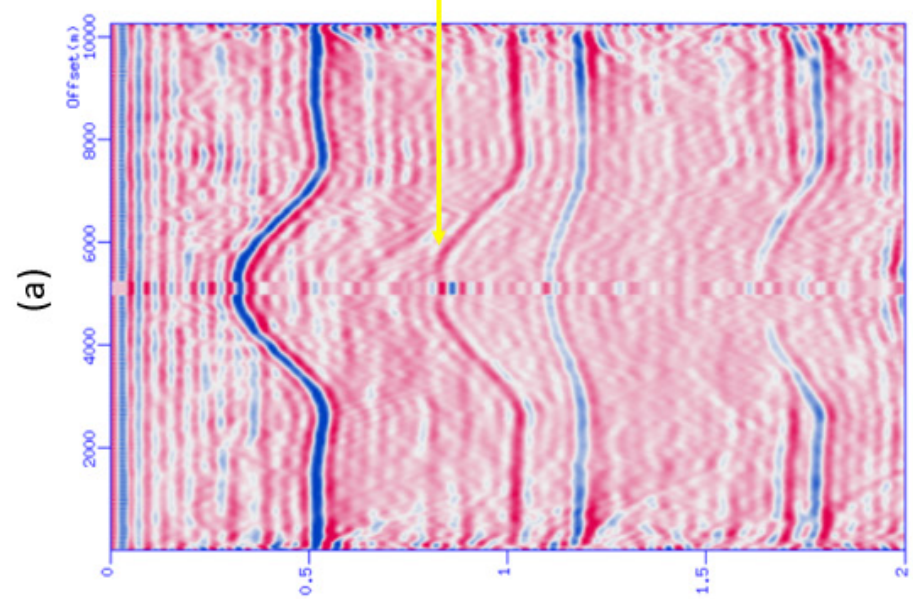

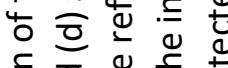

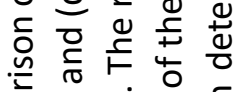

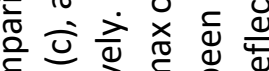

है

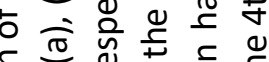

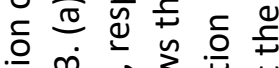

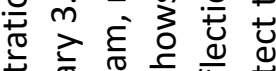

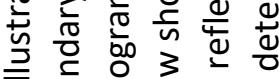

굴

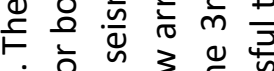

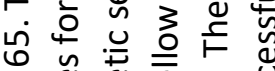

峁 嵌

该 

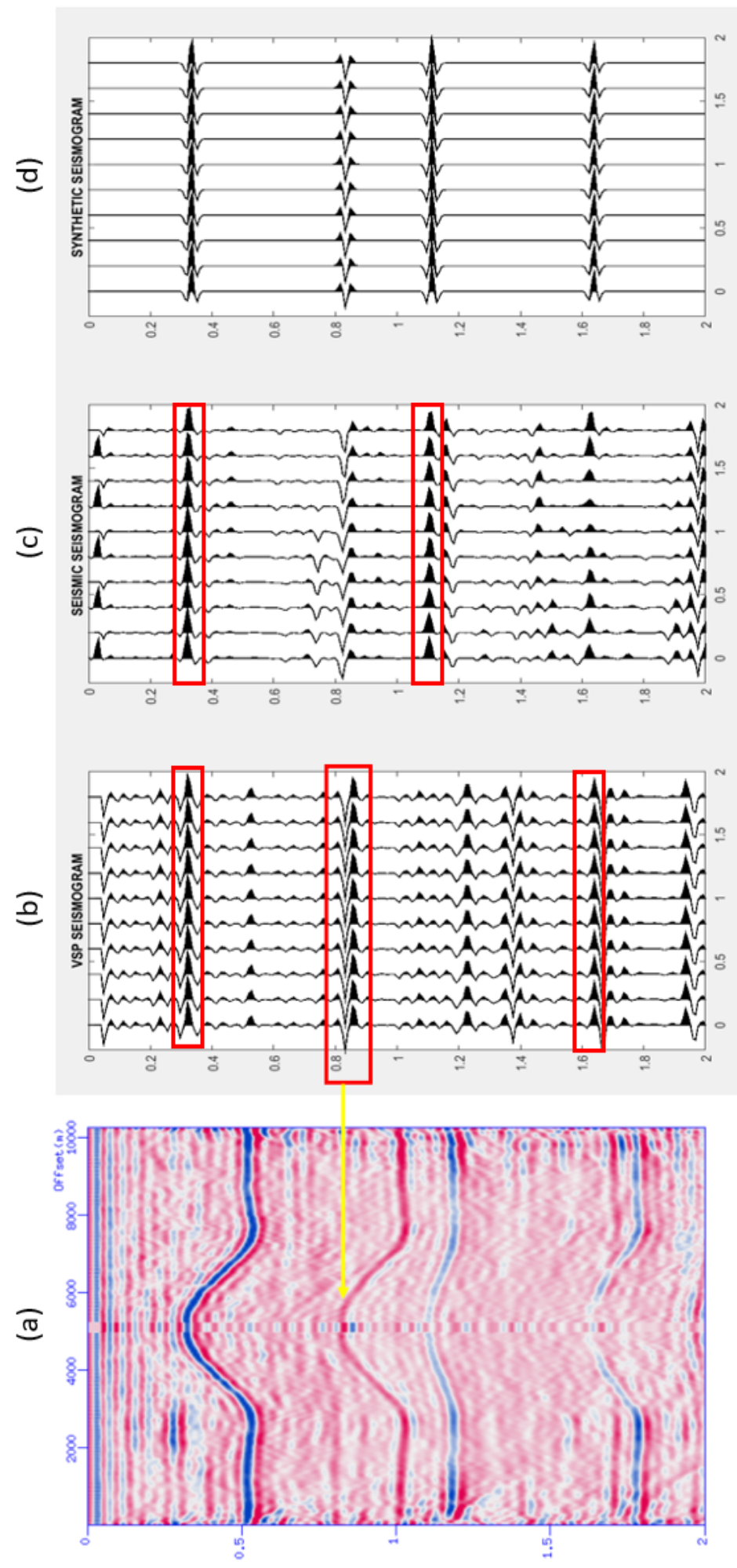

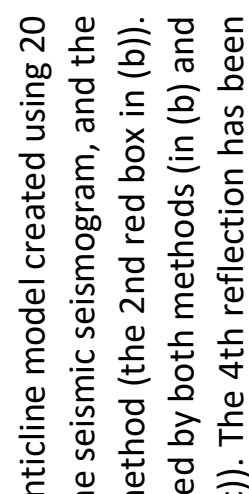

స

ए

प

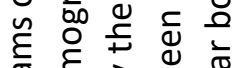

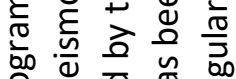

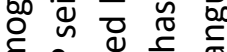

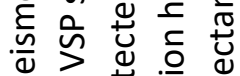

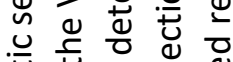

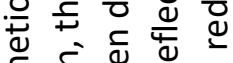

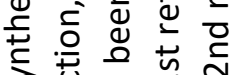

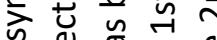

ڤ

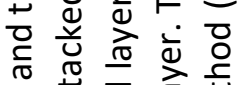

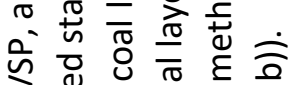

$>\stackrel{0}{0} 0$

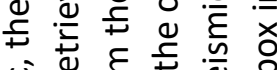

ù

है

出

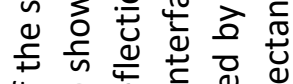

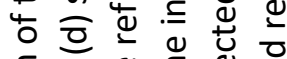

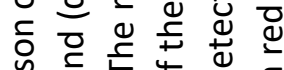

依

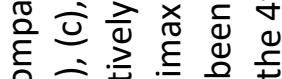

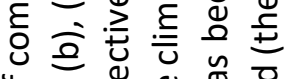

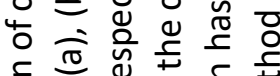

음

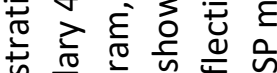

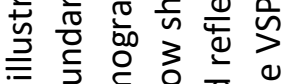

일

F

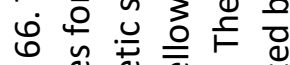

过 峁 㐫

㑢 

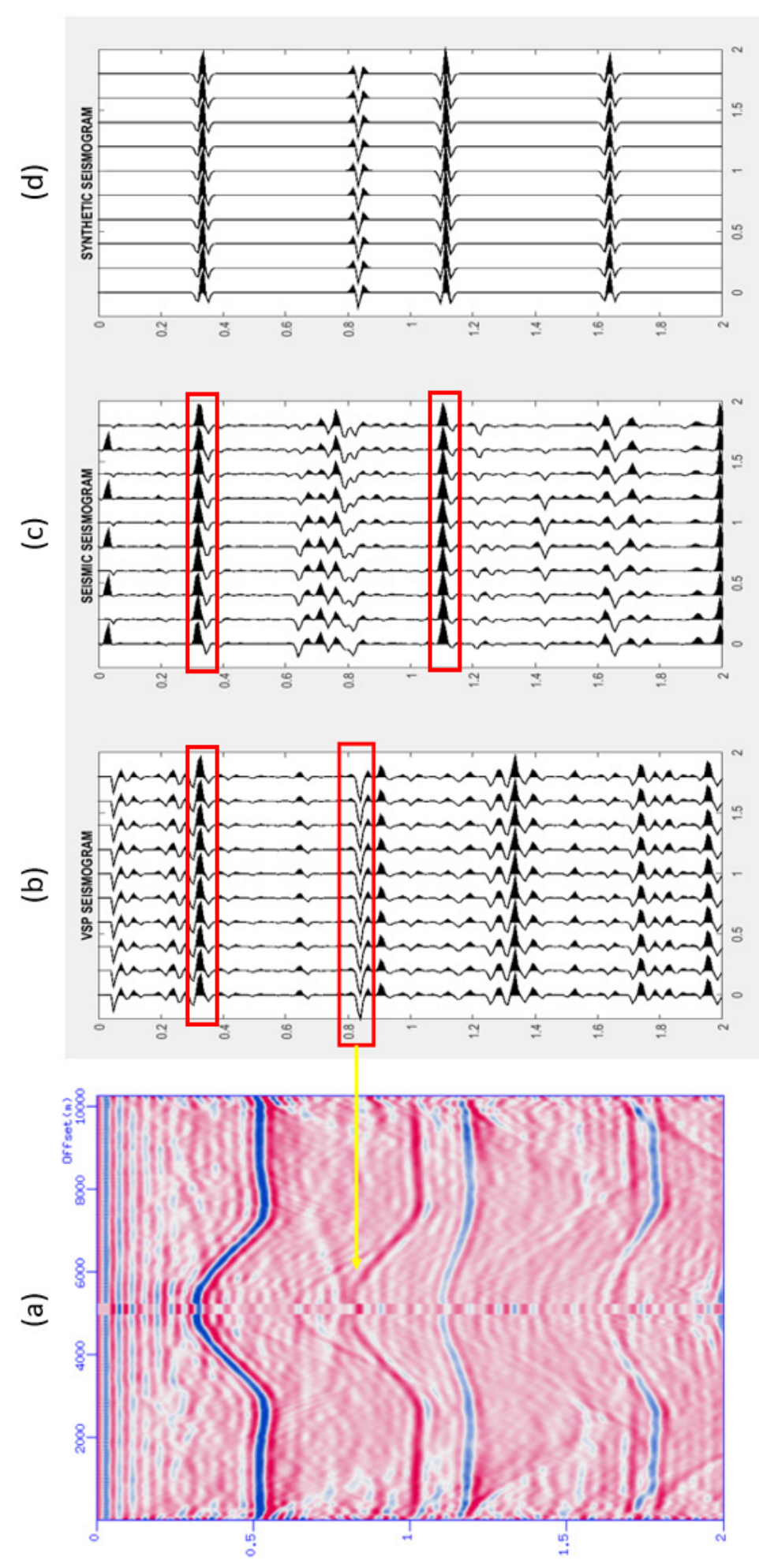

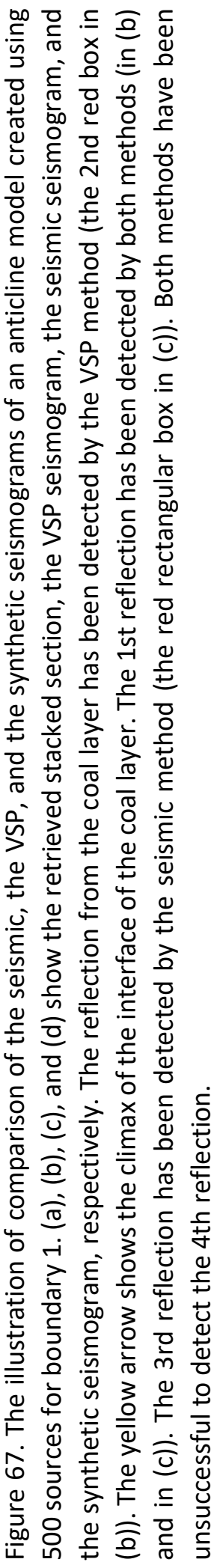



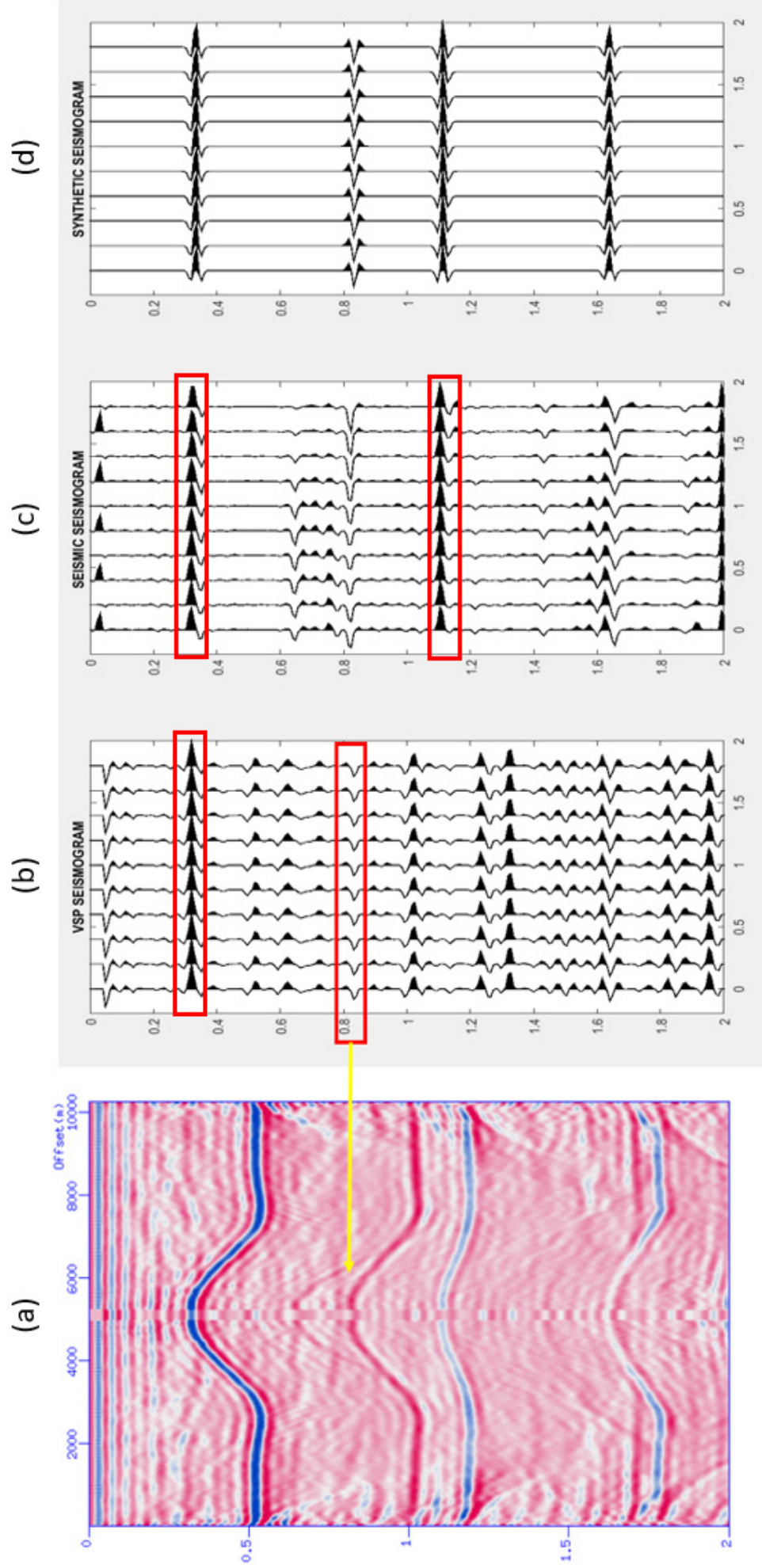

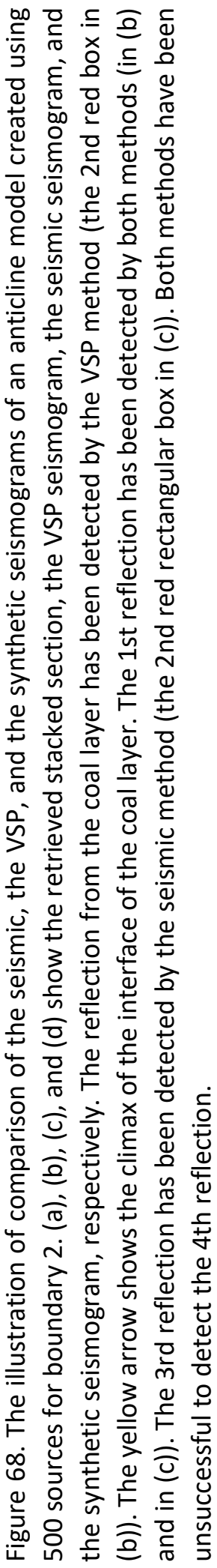



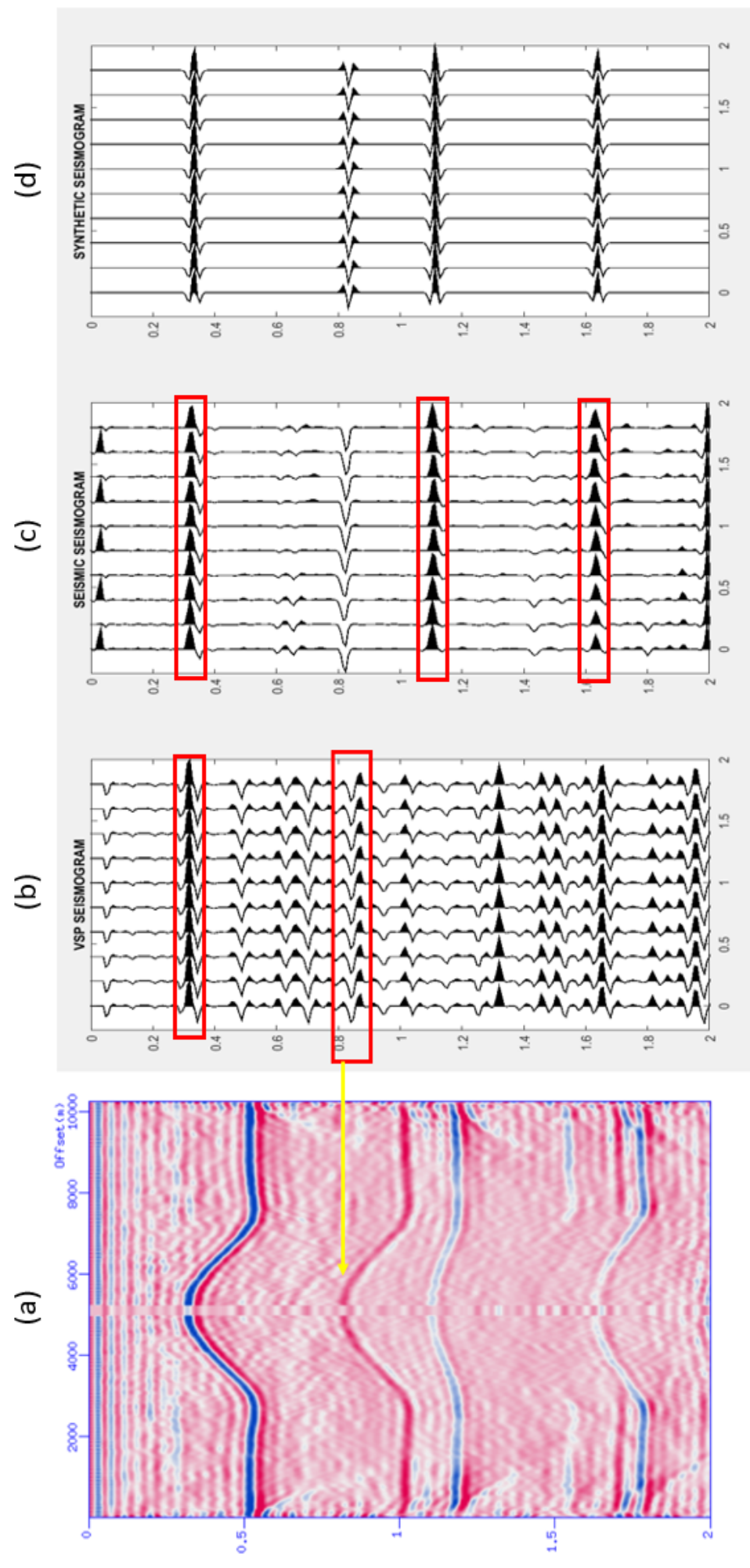

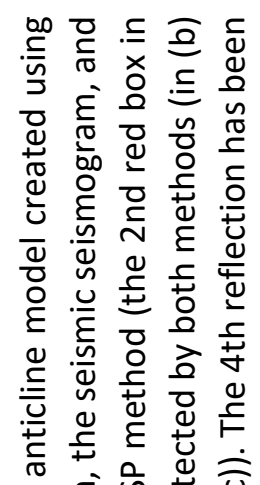

त हो जे 㫕

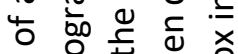

ย

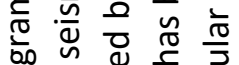

है जे प્屯

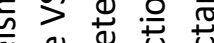

凹

U 웜

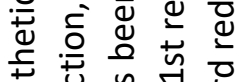

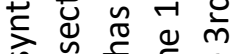

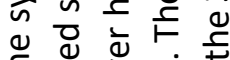

帝市市

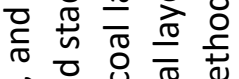

बं ठำ

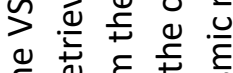

$\stackrel{ \pm}{\leftrightarrows}$

uั

है

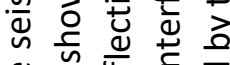

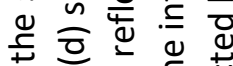

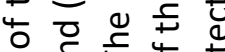

ᄃ

论

증

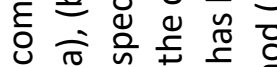

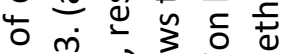

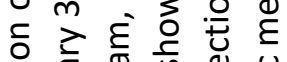

苋 元

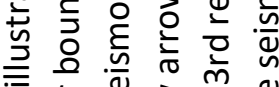

ฮั

눈 는 을

बi

Q

证 윤 

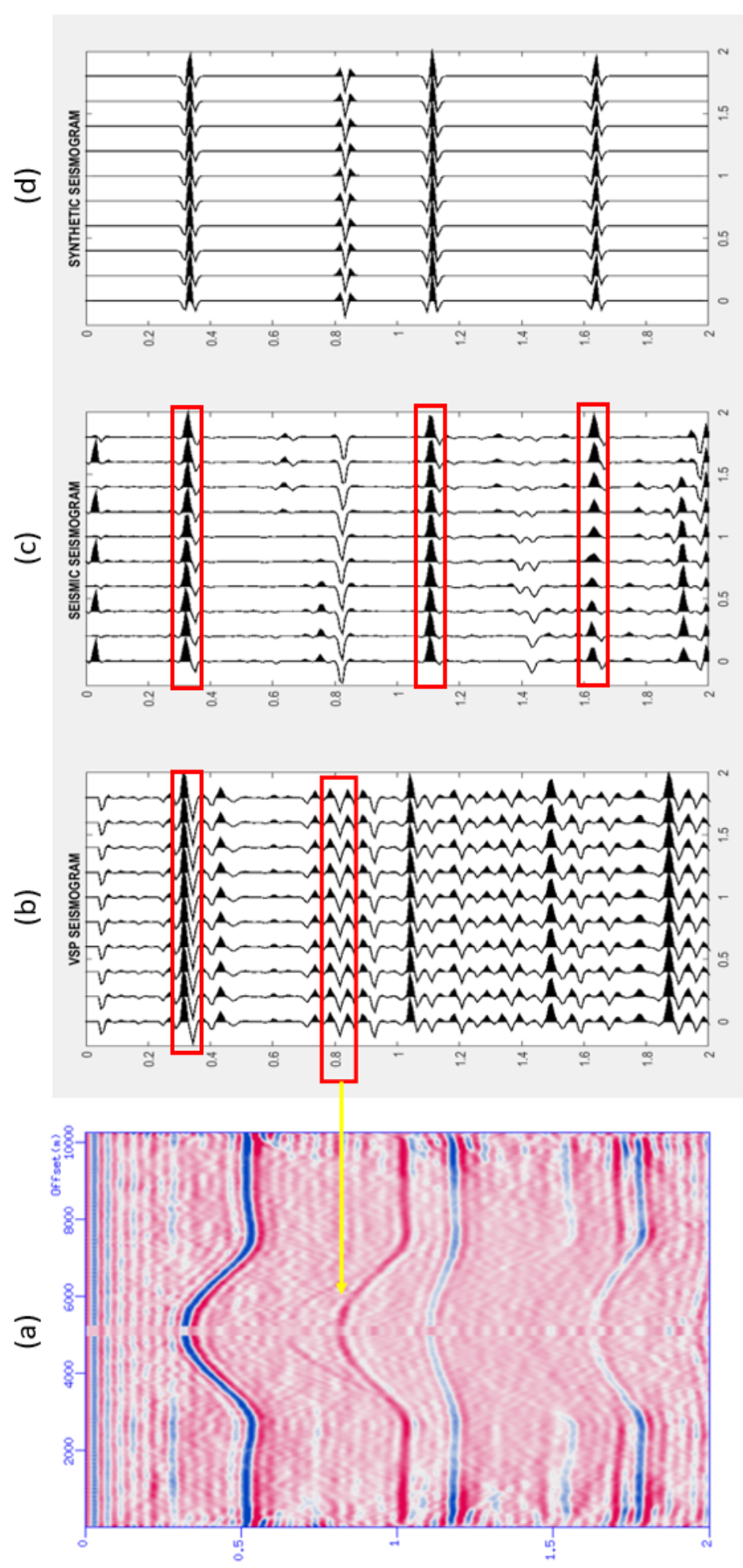

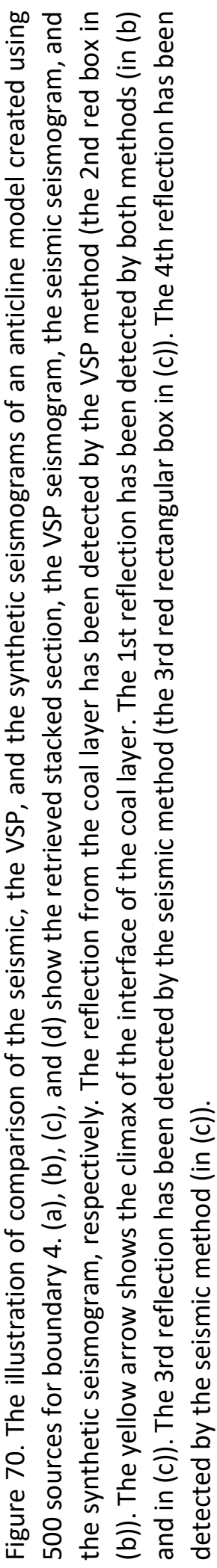



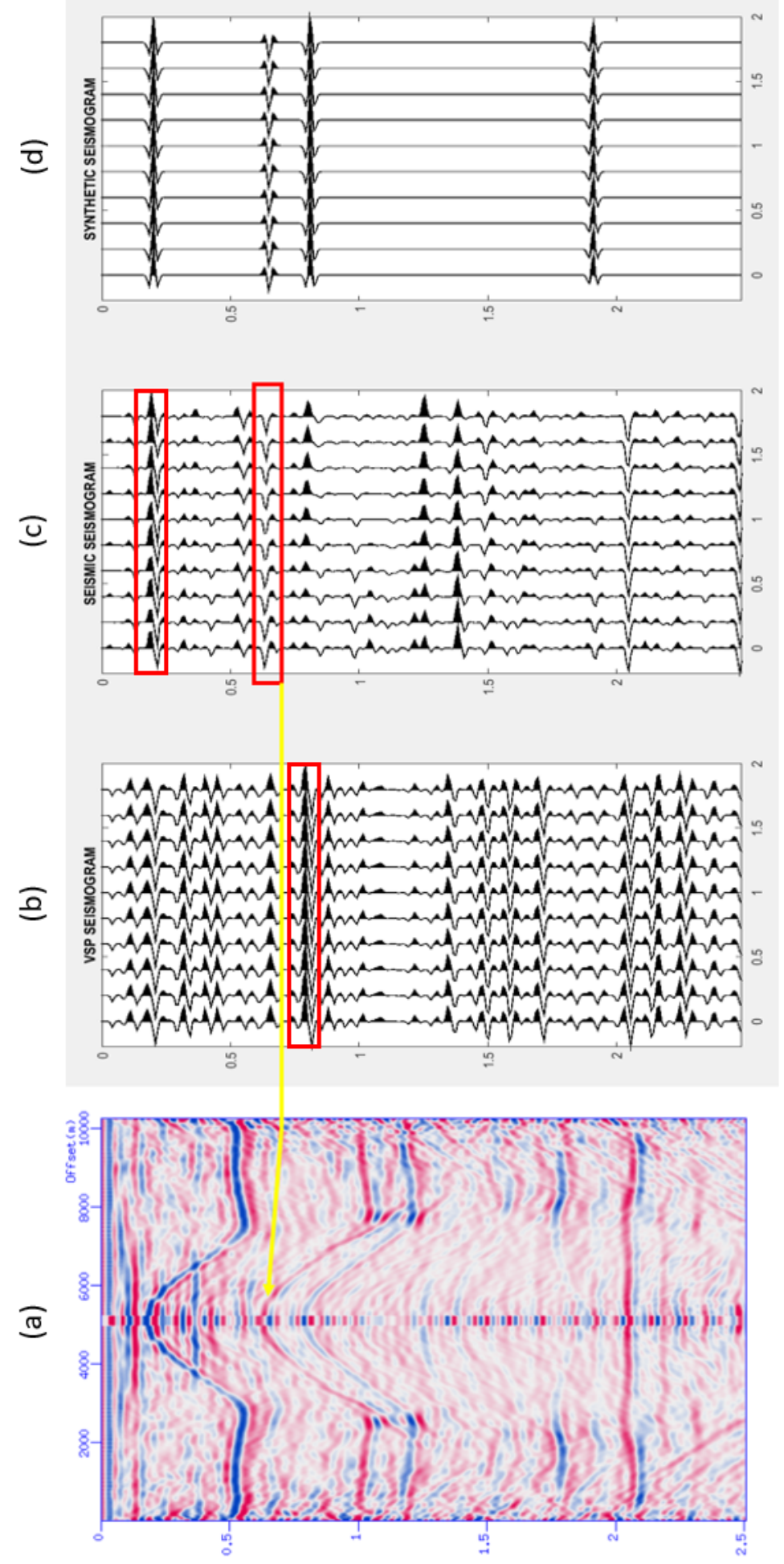

ติ

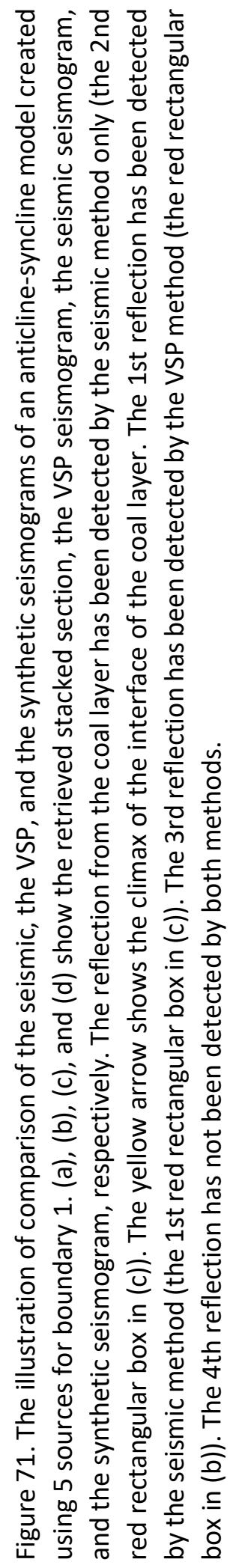



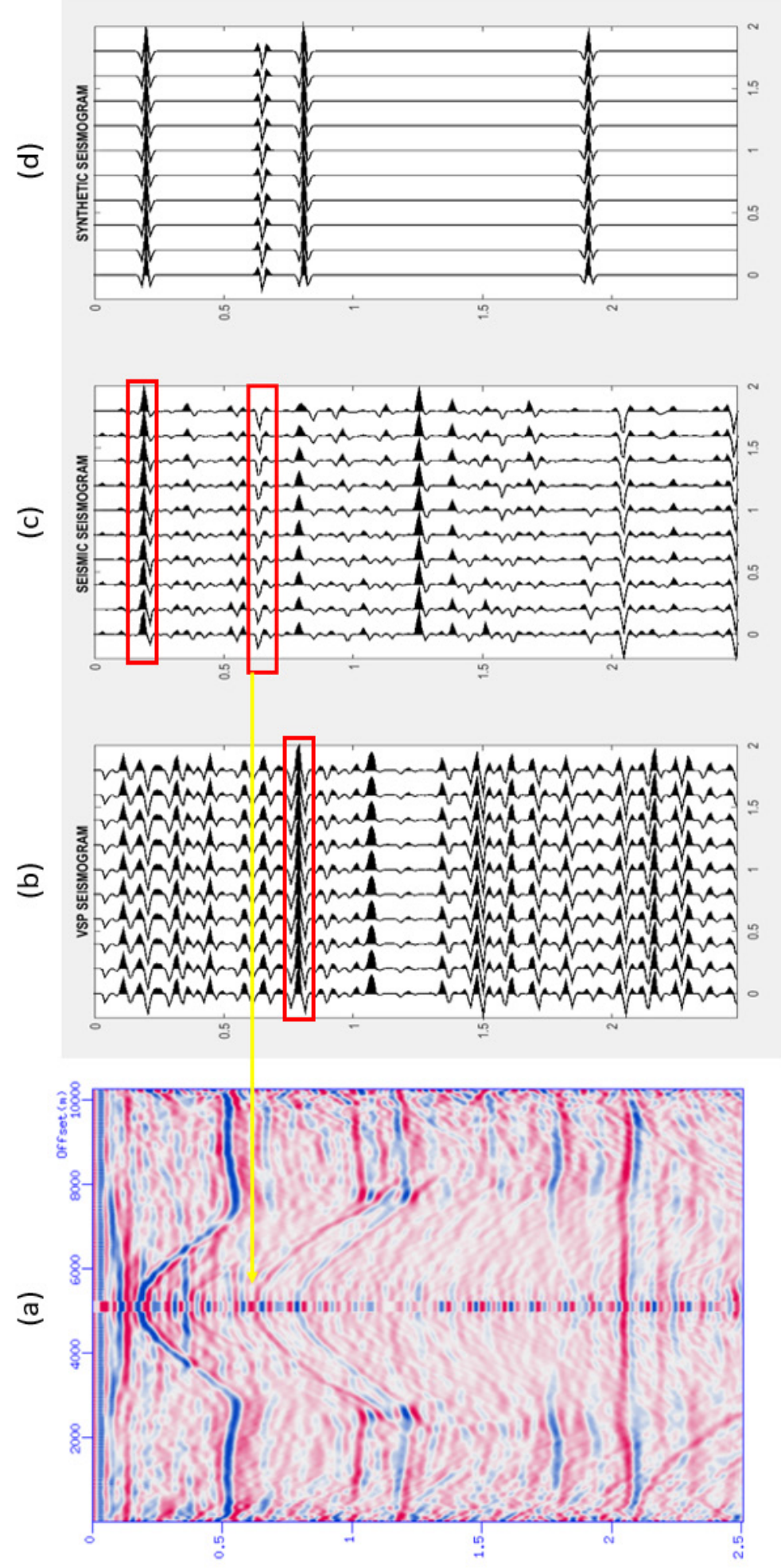

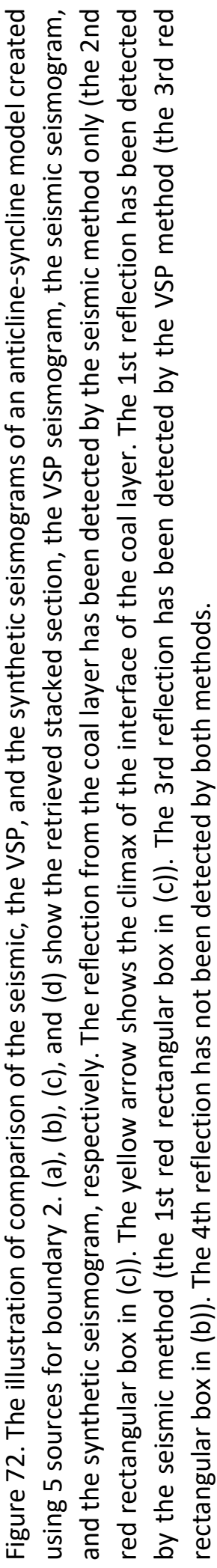



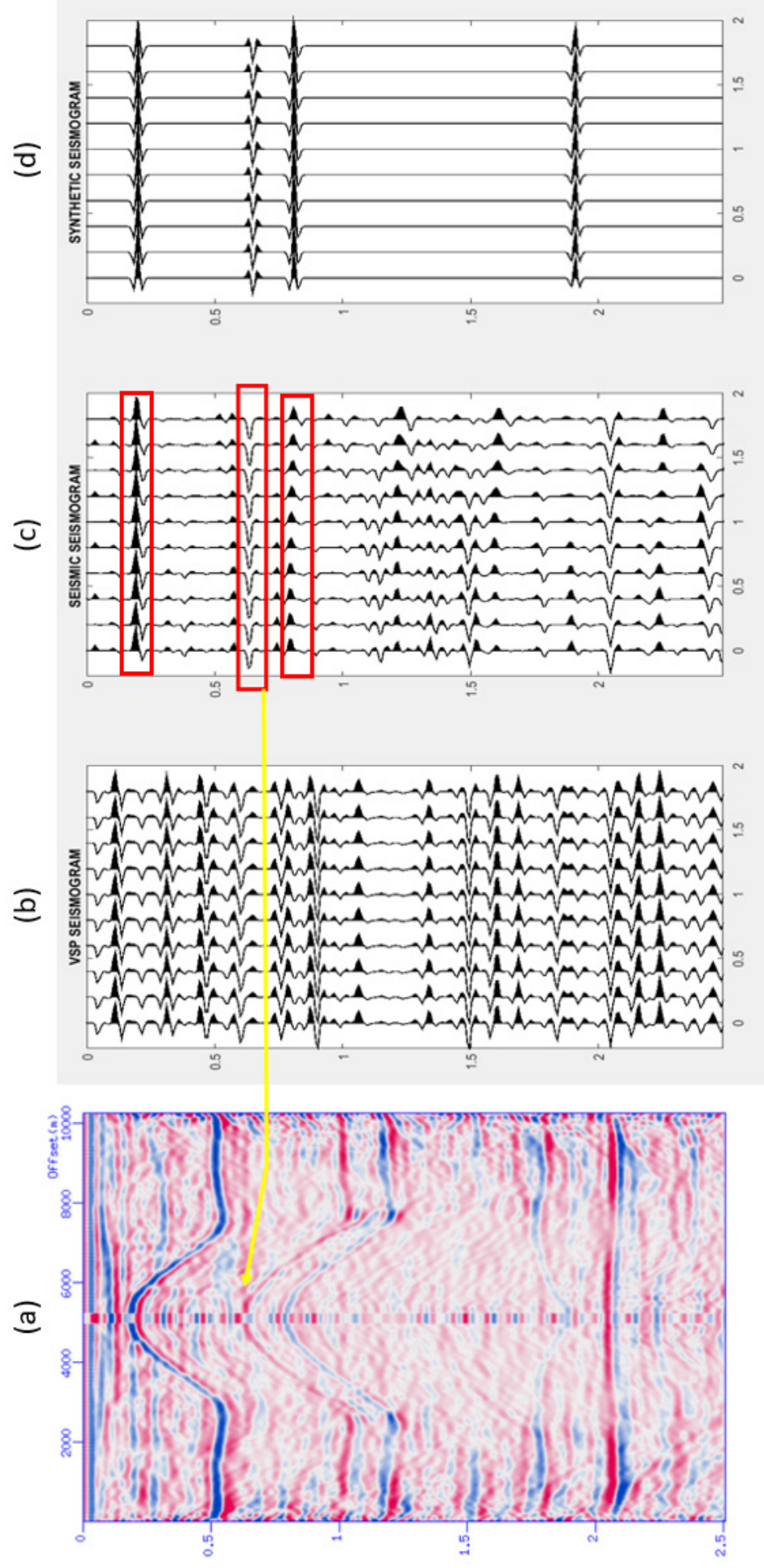

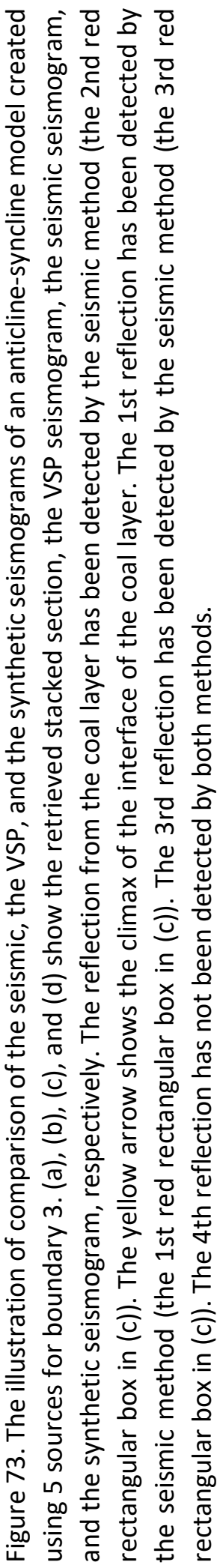




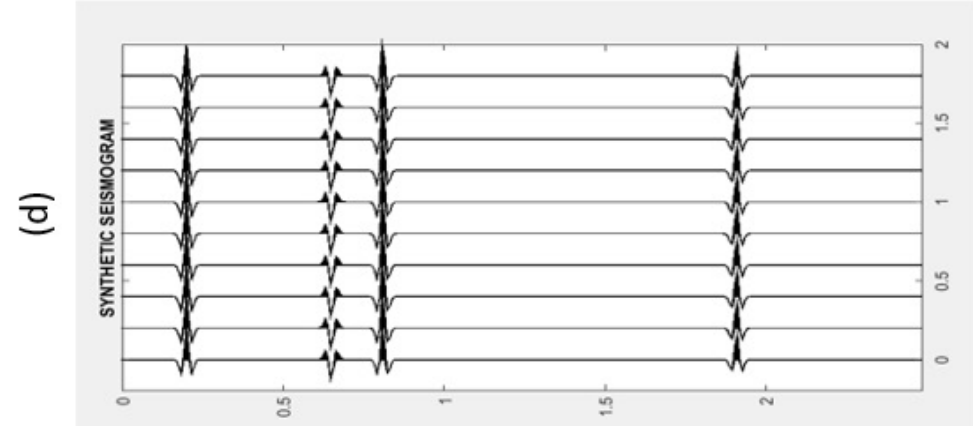

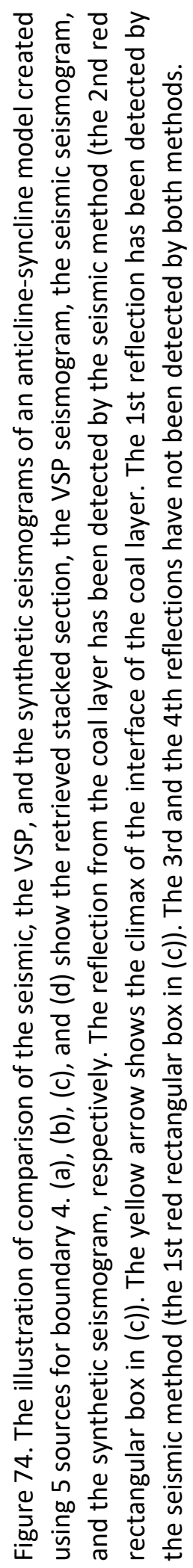

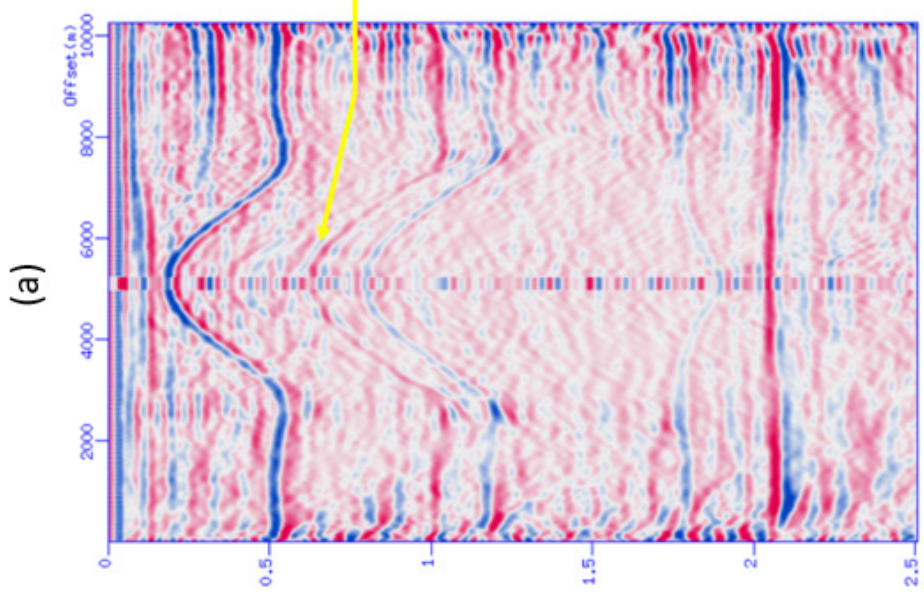




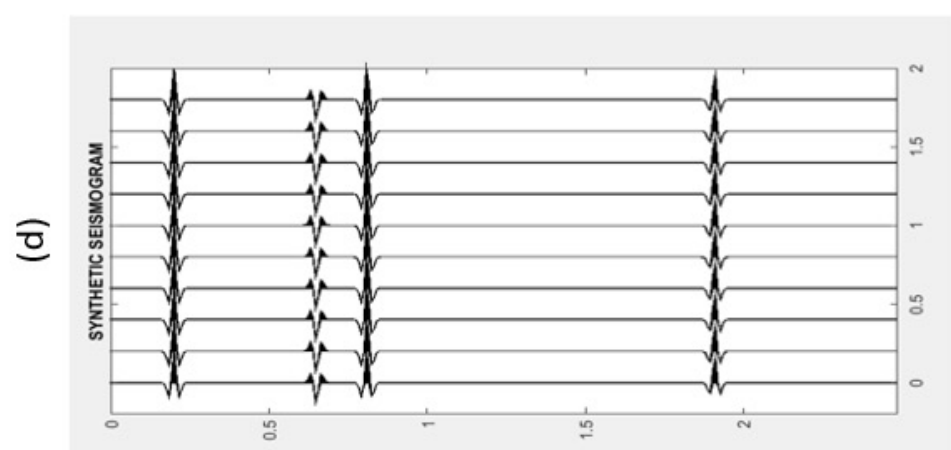

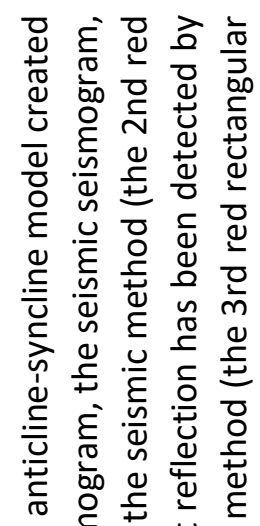

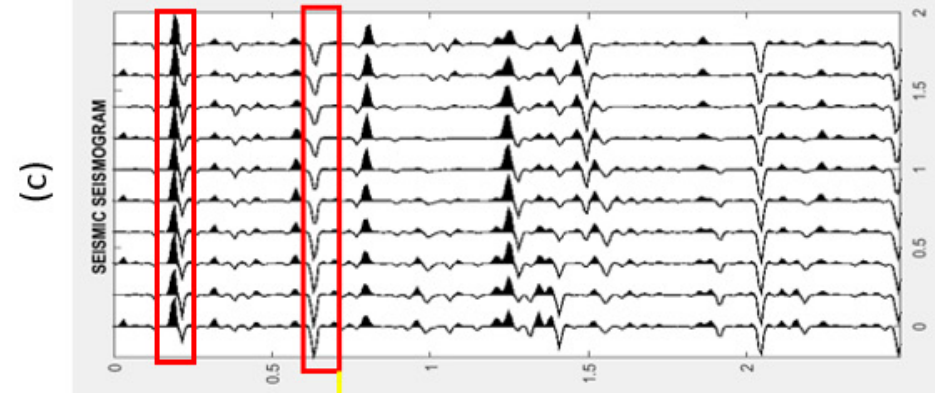

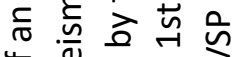

प ज व

है जे 屯્ّ

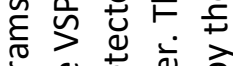

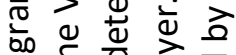

두웡

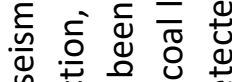

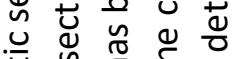

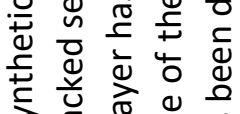

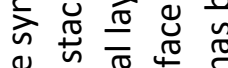
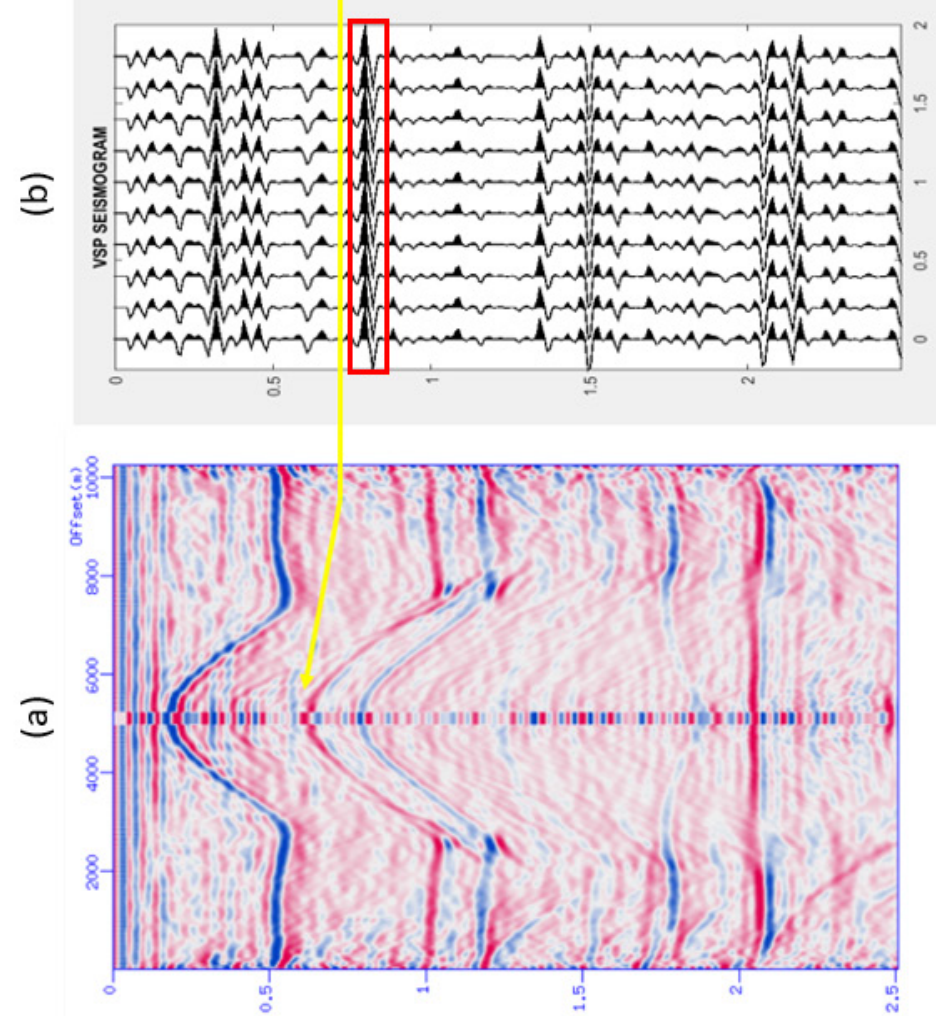

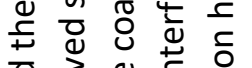

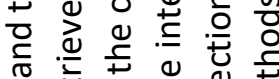

जं

ป d

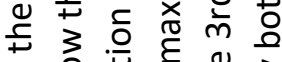

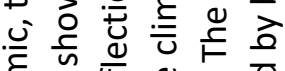

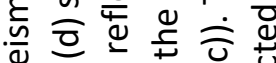

허

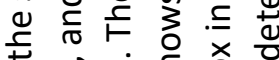

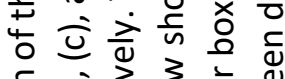

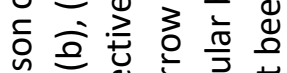

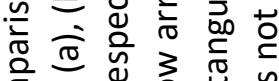

है

인 छ

ᄂ

ธ

苞

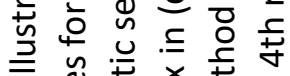

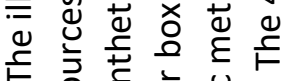

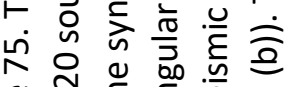

๗

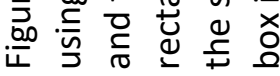



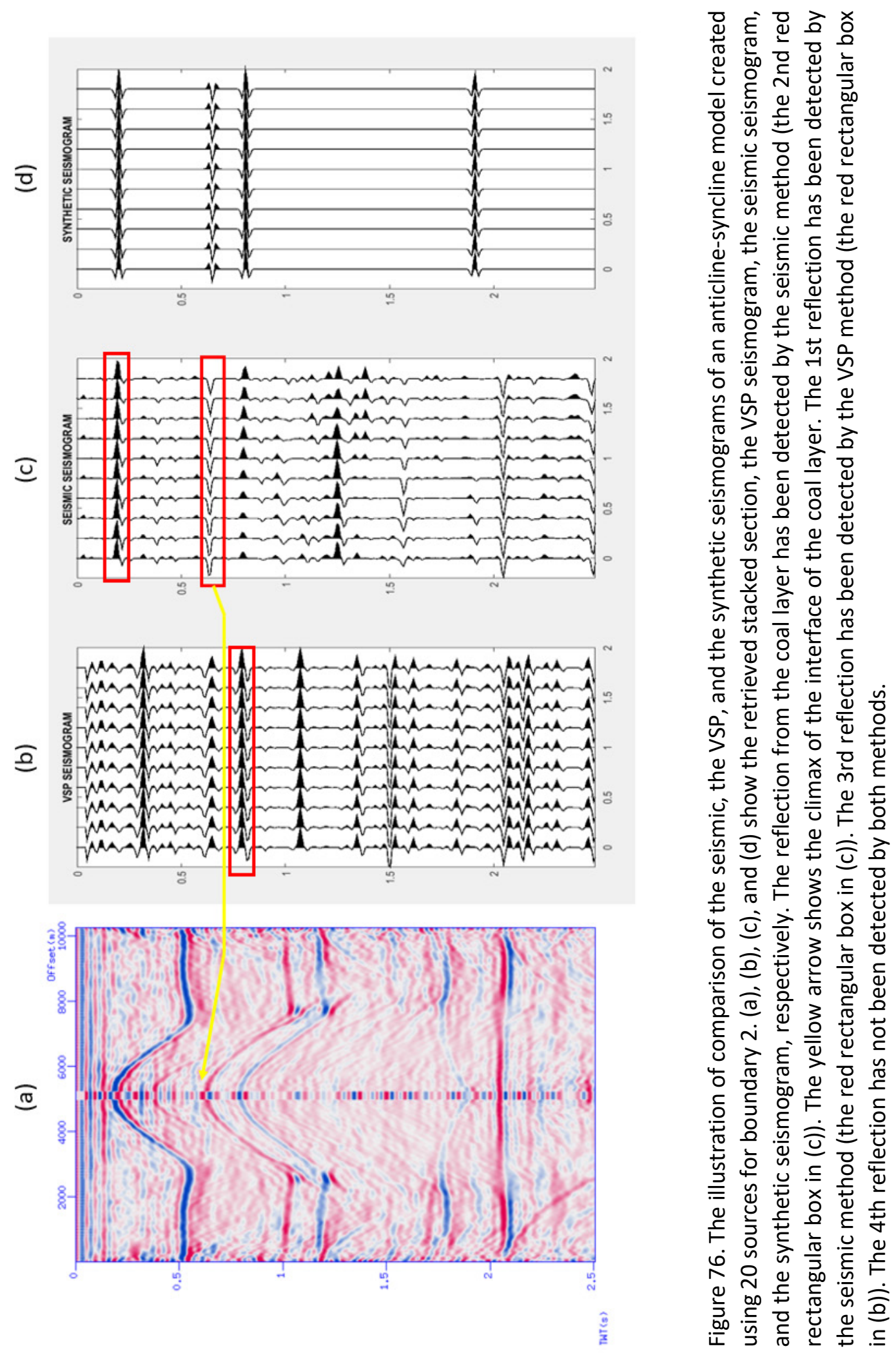

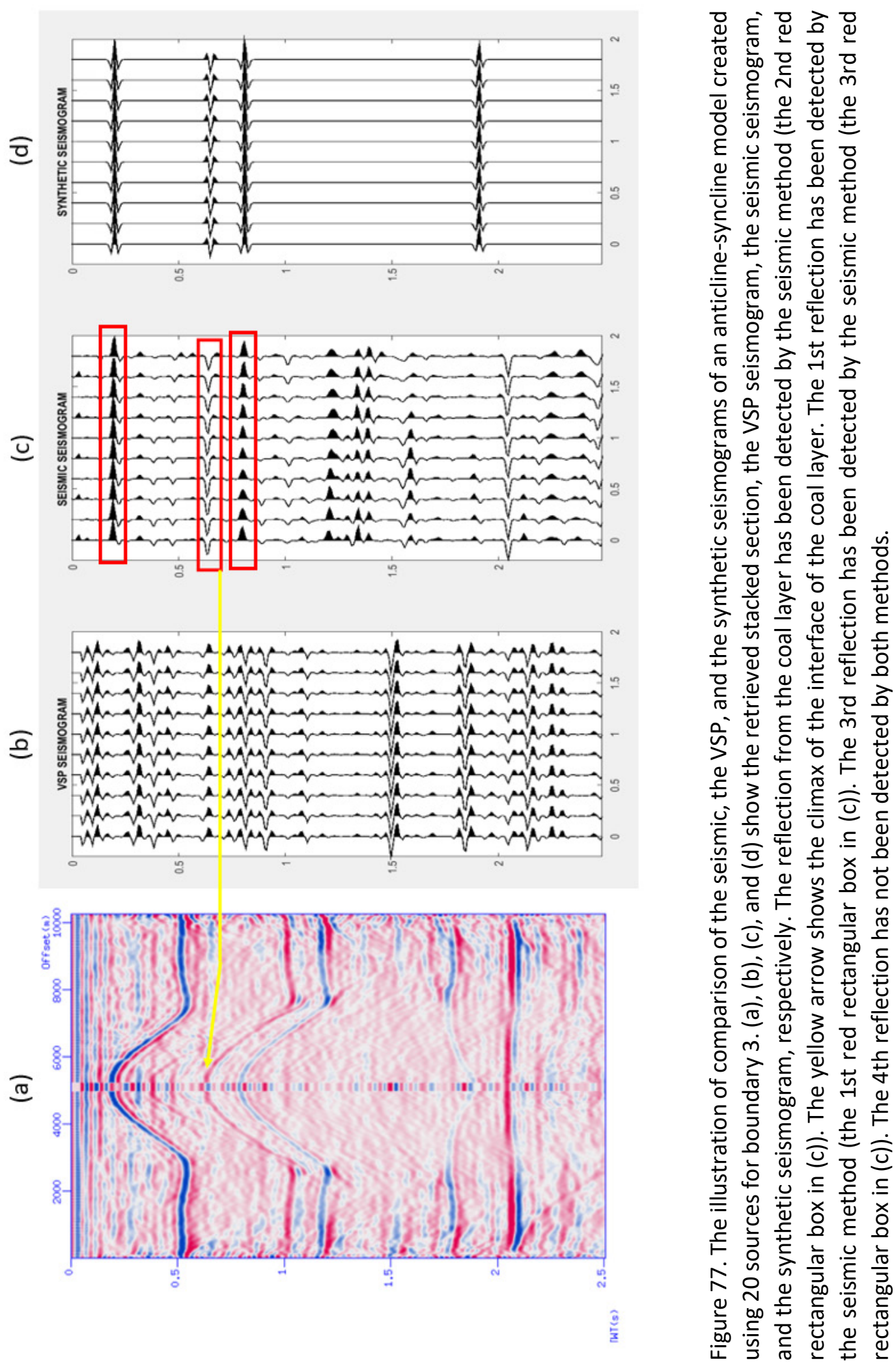

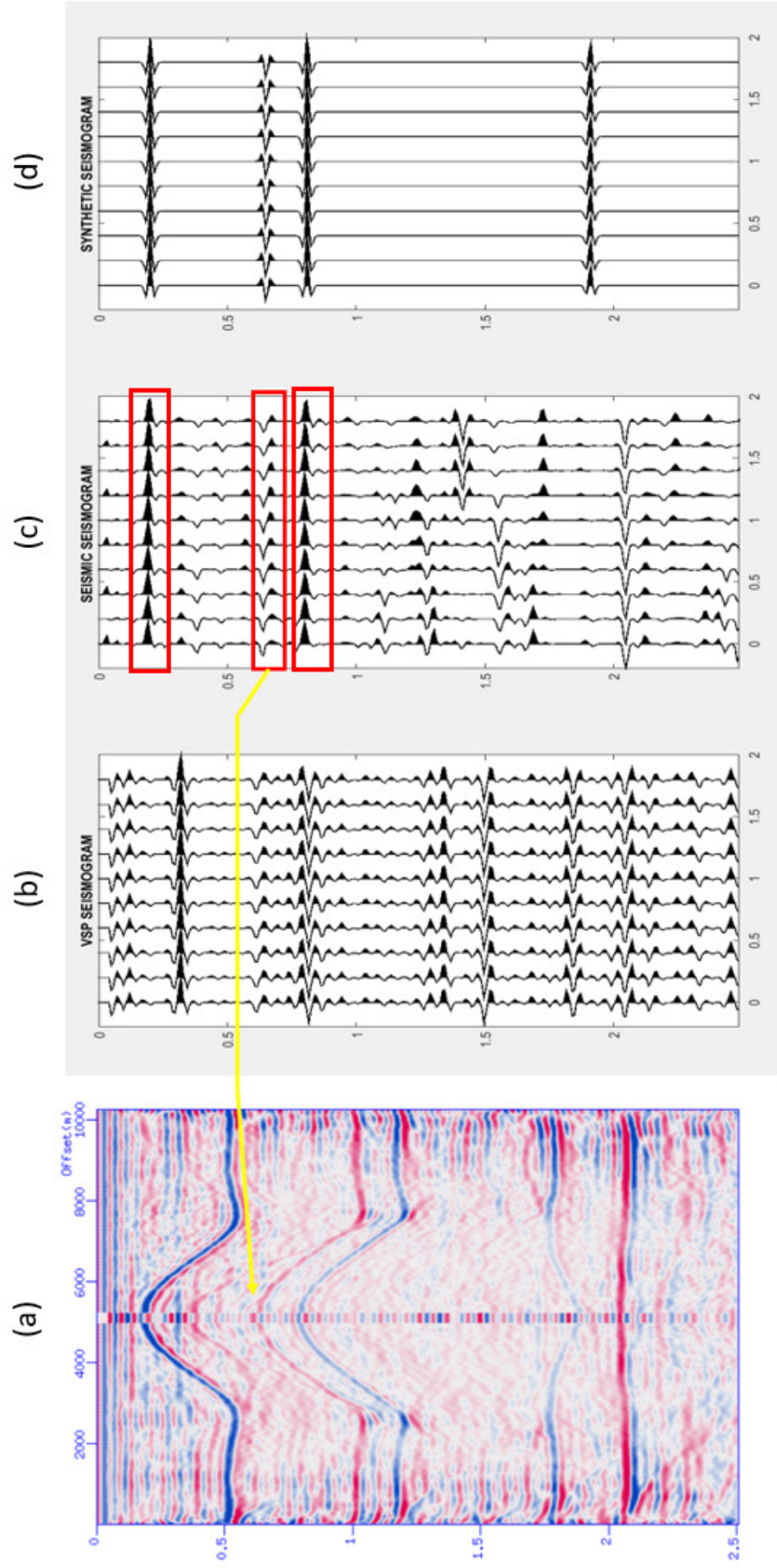

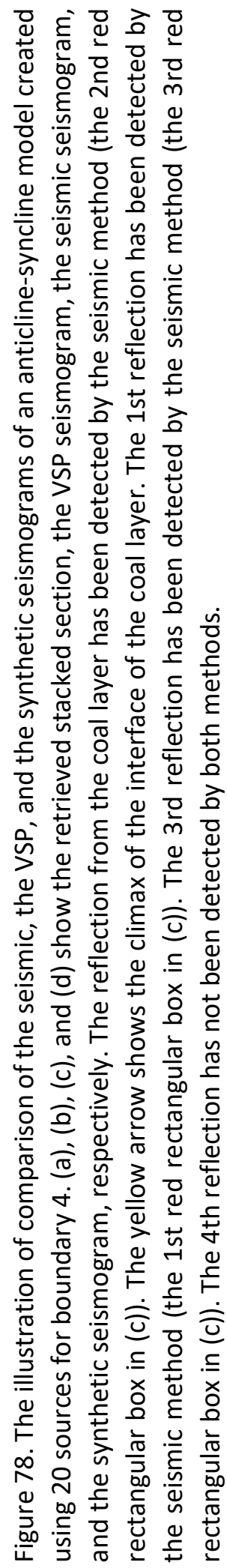



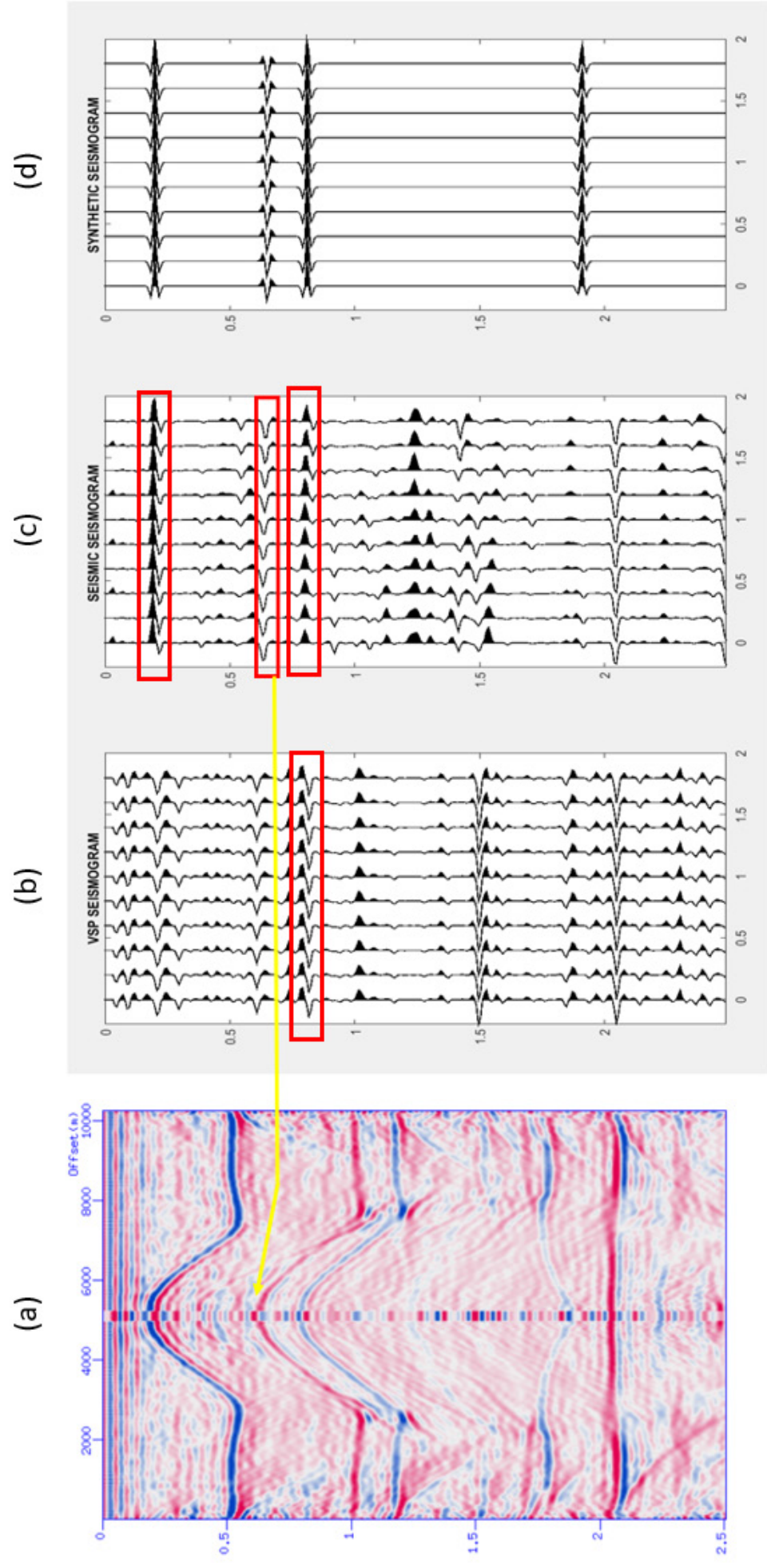

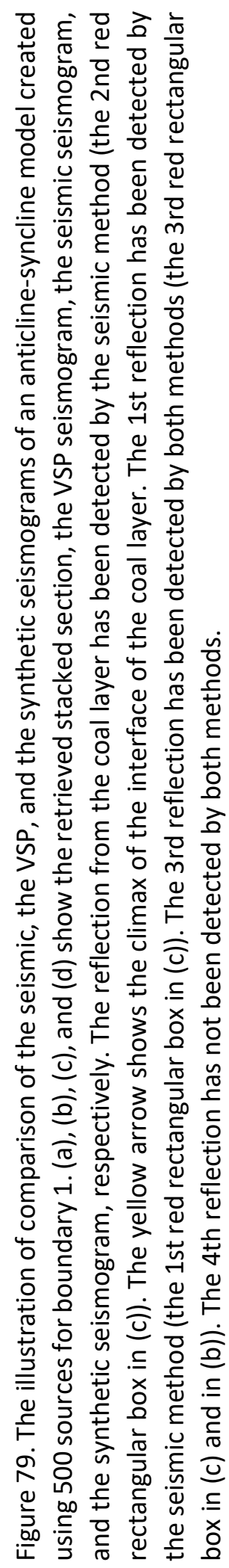




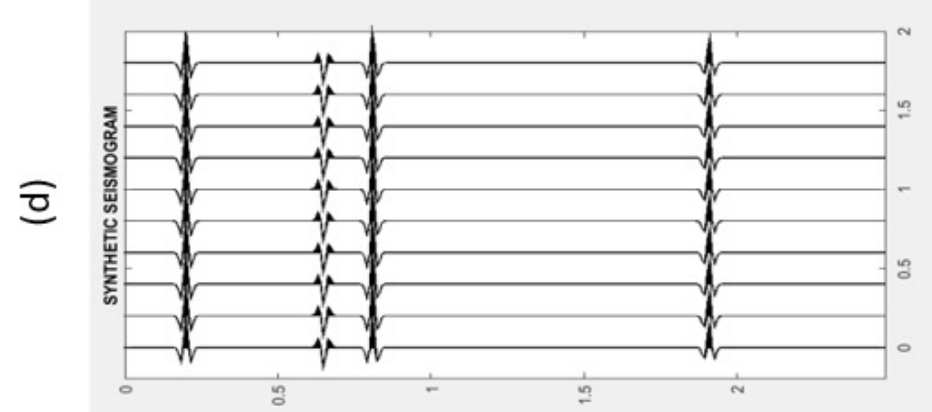

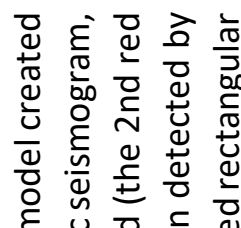

它

言竞

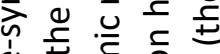

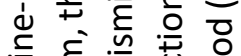

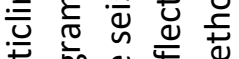

त

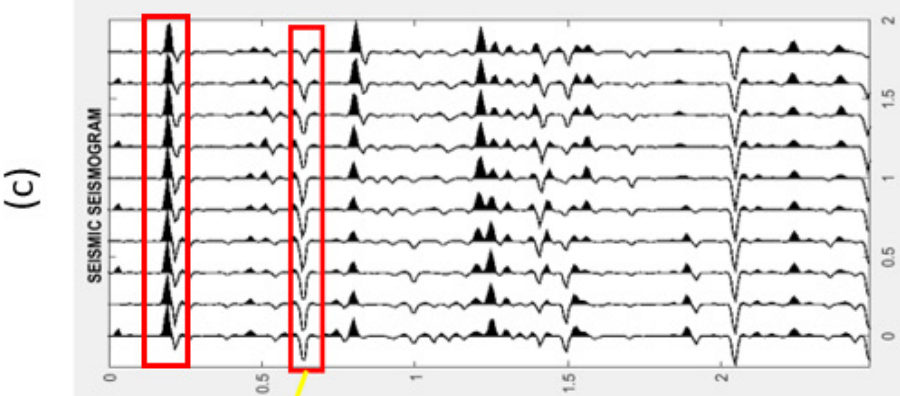

ते $\frac{E}{2}$ ठे

प०

है जे ث્屯

ข

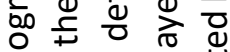

ह

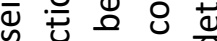

넌

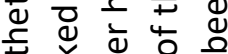

突

के

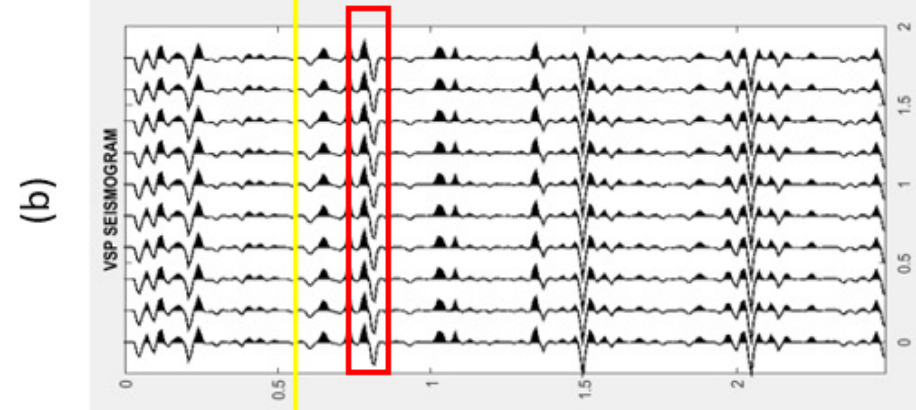

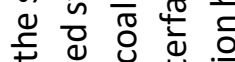

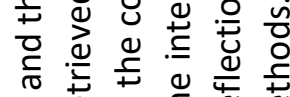

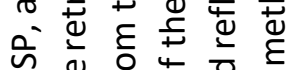

ऽ d

凹 3 을

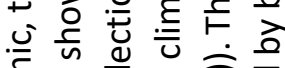

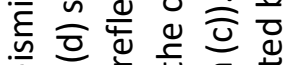

岛 ত্

凹

फ

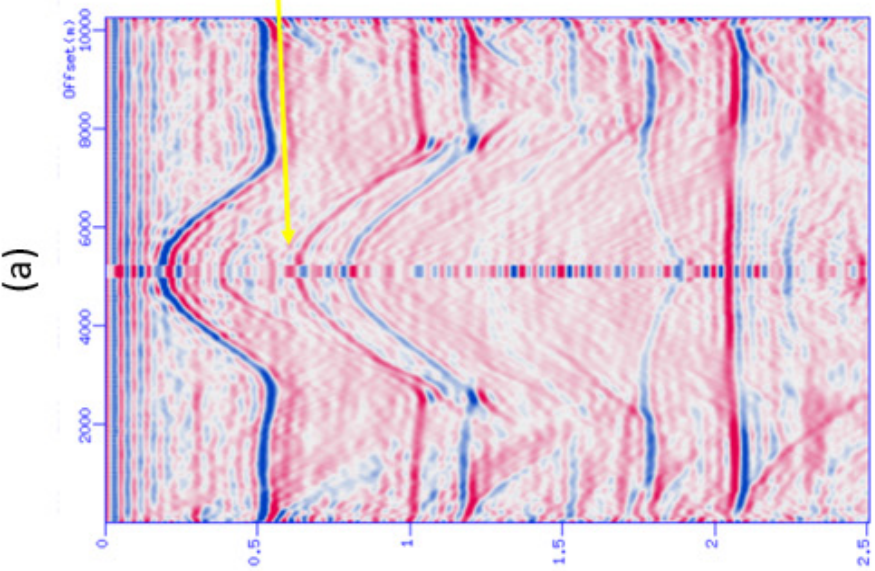

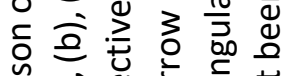

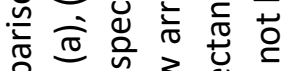

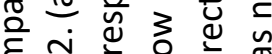

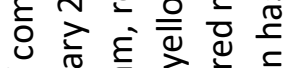

पढ त

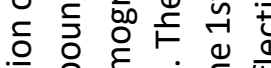

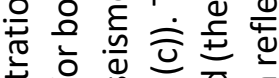

苞苍出.

ఏ d

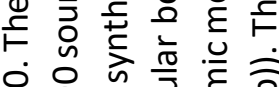

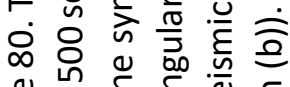

ए

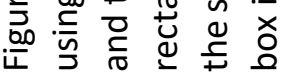




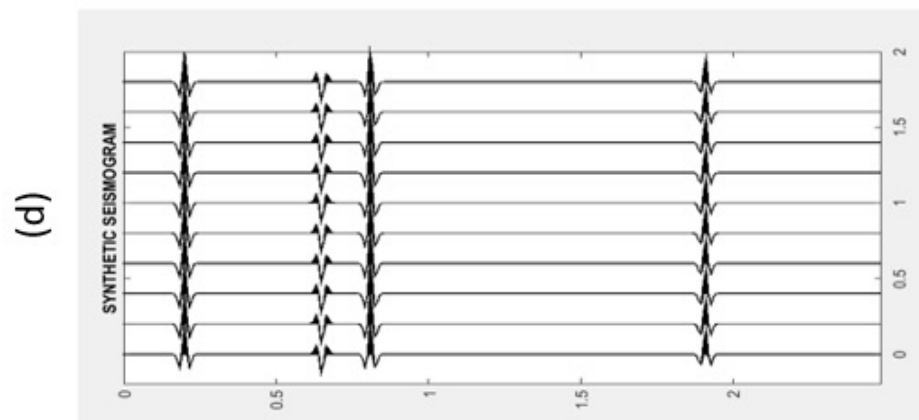

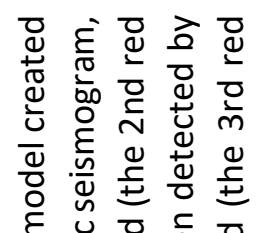

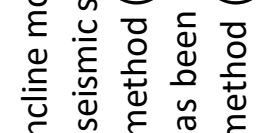

के

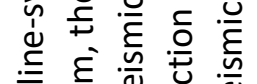
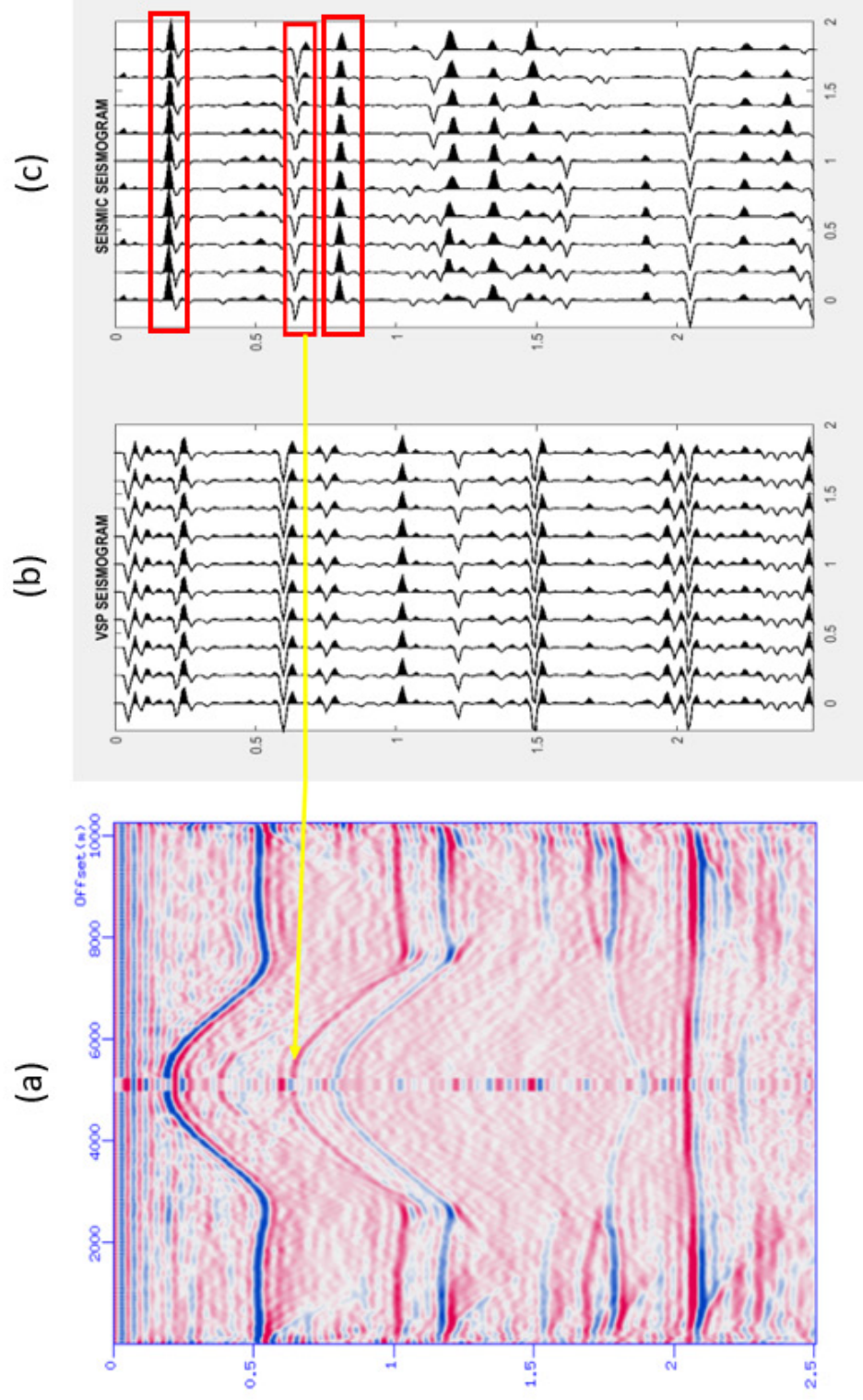

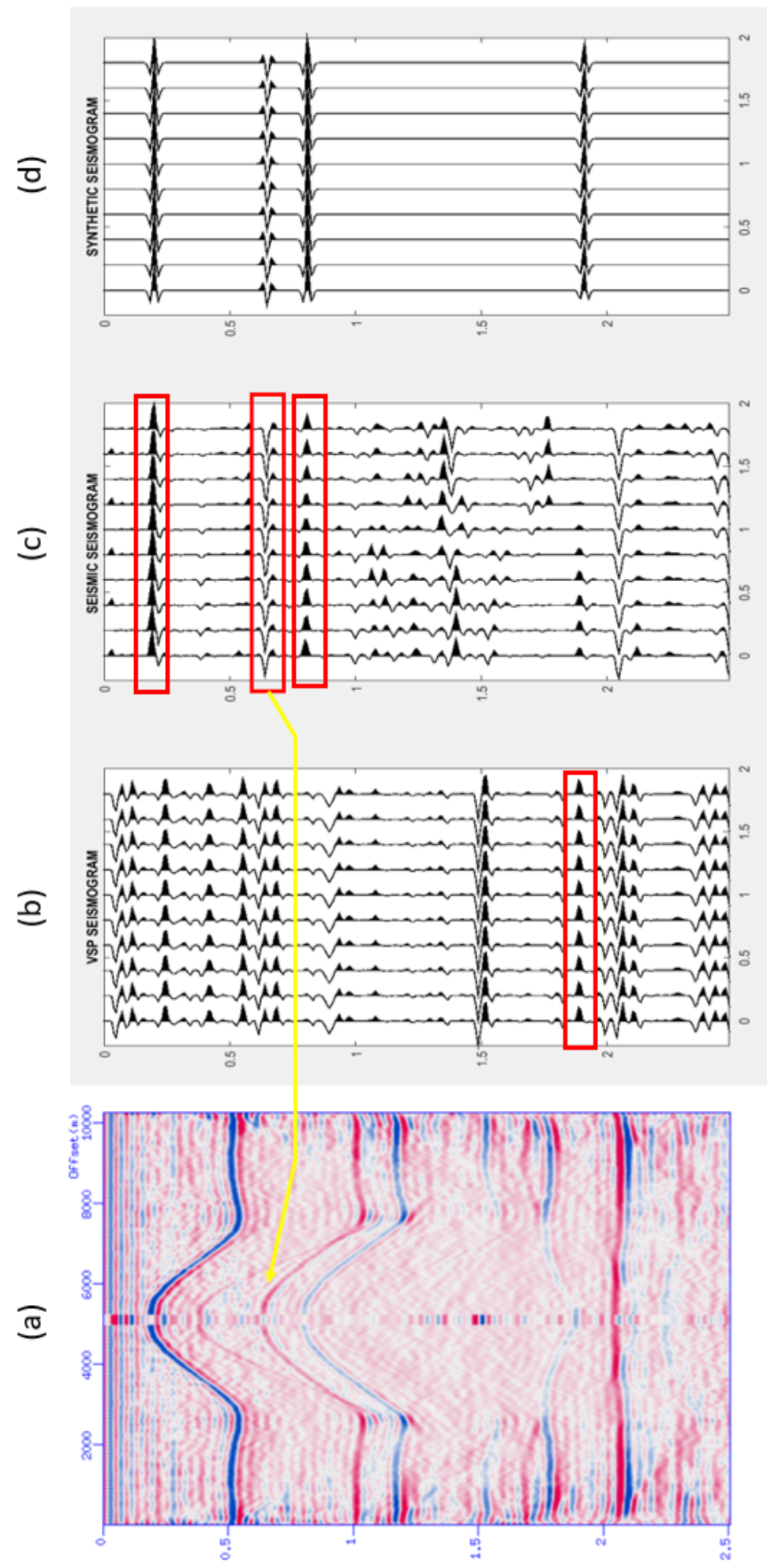

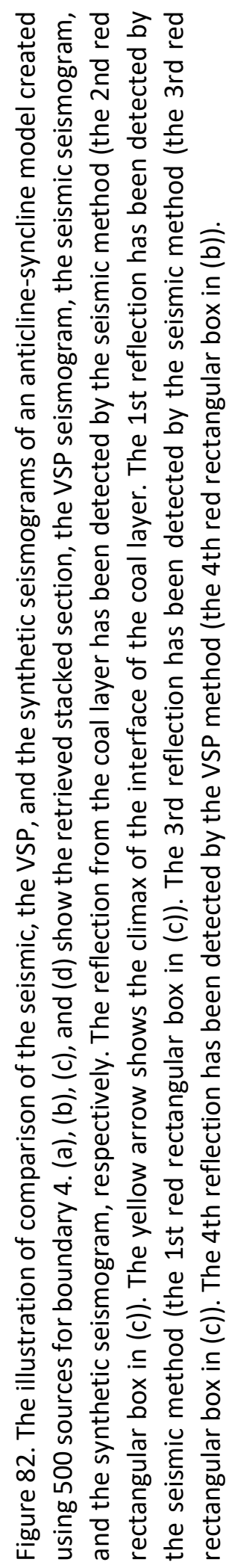




\section{Discussion}

Vertical Seismic Profiling (VSP) can be used to complement the surface seismic data acquisition in ANSI surveys. According to my results, the VSP method is sometimes helpful in determining the coal/reservoir (target layer) layer. The performance of VSP highly depends on the distribution of subsurface noises. If the noise sources are located close to the VSP borehole, in particular, below it, cross-correlation more constructively produces virtual VSP shot gathers, hence, VSP can reveal some subsurface layers. At the same time, the surface seismic renders poor results because the performance of the surface seismic highly depends a wide and homogenous distribution of seismic noises.

On the other hand, if the noise distribution is wide, the surface seismic can provide satisfactory results because not only this noise geometry can cause cross-correlation to produce more coherent virtual surface shot records, the multiple artifacts are also attenuated. At the same time, the zero-offset VSP performs weakly because this source geometry does not produce the VSP virtual shot records with a high signal to noise ratio.

While I showed some promising results of the zero-offset VSP, I would like to highlight that the VSP results would have been further convincing if more realistic simulation had been run. I did not consider the intrinsic absorption in my simulations (visco-acoustic modeling). Since in the surface seismic, way paths are longer compared to the zero-offset VSP, I expect that VSP signals should be less affected by the intrinsic absorption, and hence, have a higher quality in the real scenarios. In addition, unlike VSP, the surface seismic data acquisition always suffers from the surface waves (Rayleigh waves), and therefore, it is another factor that negatively affects the performance of the surface seismic results compared to VSP in the real ANSI surveys. 


\section{Conclusions}

Ambient noise seismic interferometry (ANSI) has been increasingly used in recent years as it utilizes underground noise sources. It can be used in challenging terrains, urban areas, and is cost-efficient compared to seismic that uses active sources such as vibroseis, dynamite, air gun, or hammer. In using these underground noise sources, numerical modeling is an important tool retrieving reflections of underground structures.

In this study, with the help of 2D finite modeling, we examined three different velocity models, which contain a model that consists of anticlines structures, flat layers, and a model consisting of anticlines and syncline, for surface seismic and zero-offset VSP methods. We created a target (coal layer) zone and tried to determine under which conditions the surface seismic method or zero-offset VSP method can detect the coal layer more successfully by using the different number of sources and different noise boundaries.

We observed that the zero-offset VSP method was generally successful in anticline model to detect the coal layer whereas the surface seismic method was more successful in the flat model and anticline-syncline model to detect the coal layer.

The increase in the number of sources and extending the noise boundaries improve the $\mathrm{S} / \mathrm{N}$ ratio of the retrieved reflections. In flat model, the surface seismic solves reflections from coal layer (the one that is shown the yellow arrow) better than the VSP method. As for the anticline model, the zero-offset VSP method solves the reflections from the coal layer (the one that is shown the yellow arrow) better than the seismic method. Finally, for the anticline-syncline model, the surface seismic method solves the reflections from coal layer (the one is shown the yellow arrow) better than the zero-offset VSP method.

In real conditions, noise distribution is not spatially wide (limited) and we would not have many sources. Also, geological layers are not flat. In a reservoir, for example, most of noise activity comes from some reservoir changes like fracturing or fault movement. Regarding our research, we concluded that when the model becomes complex, source distribution is narrow and when we have a few sources, the VSP method can help detect the layers that cannot detected by the seismic method. I propose that the VSP method may be helpful for seismic method if the geological conditions are not ideal. Generally, the surface seismic method is better but the VSP method can help to have a better insight of subsurface for complex situations. So, the zero-offset VSP method can be complementary to the surface seismic method, and it can be reached more accurate results if surface seismic and the zerooffset VSP methods used together.

Producing more and more models, using deconvolution or cross-coherence methods instead of the cross-correlation method, increasing the number of noise sources and recording times, and utilizing commercial software that can use some special processing steps that cannot be done by Seismic Unix software can lead us to better results for future studies. 


\section{References}

Bakulin, A., \& Calvert, R. (2004). Virtual source: new method for imaging and 4D below complex overburden. In SEG Technical Program Expanded Abstracts 2004 (pp. 24772480). Society of Exploration Geophysicists.

Bojarski, N. N. (1983). Generalized reaction principles and reciprocity theorems for the wave equations, and the relationship between the time-advanced and time-retarded fields. The Journal of the Acoustical Society of America, 74(1), 281-285.

Boullenger, B., Verdel, A., Paap, B., Thorbecke, J., \& Draganov, D. (2015). Studying CO 2 storage with ambient-noise seismic interferometry: A combined numerical feasibility study and field-data example for Ketzin, Germany. Geophysics, 80(1), Q1-Q13.

Brenguier, F., Shapiro, N. M., Campillo, M., Ferrazzini, V., Duputel, Z., Coutant, O., \& Nercessian, A. (2008). Towards forecasting volcanic eruptions using seismic noise. Nature Geoscience, 1(2), 126-130.

Campillo, M., \& Paul, A. (2003). Long-range correlations in the diffuse seismic coda. Science, 299(5606), 547-549.

Cao, H., \& Askari, R. (2019). Comparison of seismic interferometry techniques for the retrieval of seismic body waves in $\mathrm{CO} 2$ sequestration monitoring. Journal of Geophysics and Engineering, 16(6), 1094-1115.

Chen, Y., Li, Y., Zhao, H., Gao, X., \& Qiu, Y. (2013, July). Study of first arrival time of VSP data. In Near Surface Geophysics Asia Pacific Conference, Beijing, China 17-19 July 2013 (pp. 97-101). Society of Exploration Geophysicists, Australian Society of Exploration Geophysicists, Chinese Geophysical Society, Korean Society of Earth and Exploration Geophysicists, and Society of Exploration Geophysicists of Japan.

Claerbout, J. F. (1968). Synthesis of a layered medium from its acoustic transmission response. Geophysics, 33(2), 264-269.

Curtis, A. (2009). Source-receiver seismic interferometry. In SEG Technical Program Expanded Abstracts 2009 (pp. 3655-3659). Society of Exploration Geophysicists.

Curtis, A., \& Halliday, D. (2010). Source-receiver wave field interferometry. Physical Review E, 81(4), 046601.

Curtis, A., Gerstoft, P., Sato, H., Snieder, R., \& Wapenaar, K. (2006). Seismic interferometry-Turning noise into signal. The Leading Edge, 25(9), 1082-1092.

Derode, A., Larose, E., Campillo, M., \& Fink, M. (2003). How to estimate the Green's function of a heterogeneous medium between two passive sensors? Application to acoustic waves. Applied Physics Letters, 83(15), 3054-3056. 
Derode, A., Larose, E., Tanter, M., De Rosny, J., Tourin, A., Campillo, M., \& Fink, M. (2003). Recovering the Green's function from field-field correlations in an open scattering medium (L). The Journal of the Acoustical Society of America, 113(6), 2973-2976.

Draganov, D., Campman, X., Thorbecke, J., Verdel, A., \& Wapenaar, K. (2009). Reflection images from ambient seismic noise. Geophysics, 74(5), A63-A67.

Draganov, D., Wapenaar, K., Mulder, W., Singer, J., \& Verdel, A. (2007). Retrieval of reflections from seismic background-noise measurements. Geophysical Research Letters, 34(4).

Fichtner, A., Stehly, L., Ermert, L., \& Boehm, C. (2016). Generalised interferometry-I. Theory for inter-station correlations. Geophysical Journal International, ggw420.

He, R., Hornby, B., \& Schuster, G. (2006). 3D wave-equation interferometric migration of VSP multiples. In SEG Technical Program Expanded Abstracts 2006 (pp. 3442-3446). Society of Exploration Geophysicists.

Hohl, D., \& Mateeva, A. (2006). Passive seismic reflectivity imaging with ocean-bottom cable data. In SEG Technical Program Expanded Abstracts 2006 (pp. 1560-1564). Society of Exploration Geophysicists.

Hornby, B. E., \& Yu, J. (2007). Interferometric imaging of a salt flank using walkaway VSP data. The Leading Edge, 26(6), 760-763.

Jiang, Z., Sheng, J., Yu, J., Schuster, G. T., \& Hornby, B. E. (2007). Migration methods for imaging different-order multiples. Geophysical Prospecting, 55(1), 1-19.

Kumar, M. R., \& Bostock, M. G. (2006). Transmission to reflection transformation of teleseismic wavefields. Journal of Geophysical Research: Solid Earth, 111(B8).

Larose, E., Margerin, L., Derode, A., van Tiggelen, B., Campillo, M., Shapiro, N., ... \& Tanter, M. (2006). Correlation of random wavefields: An interdisciplinary review. Geophysics, 71(4), SI11-SI21.

Lobkis, O. I., \& Weaver, R. L. (2001). On the emergence of the Green's function in the correlations of a diffuse field. The Journal of the Acoustical Society of America, 110(6), 3011-3017.

Lu, R., Willis, M., Campman, X., Ajo-Franklin, J., \& Toksöz, M. N. (2008). Redatuming through a salt canopy and target-oriented salt-flank imaging. Geophysics, 73(3), S63-S71.

Lumley, D. E. (2001). Time-lapse seismic reservoir monitoring. Geophysics, 66(1), 50-53. 
Matsushima, J., Ali, M. Y., \& Bouchaala, F. (2016). Seismic attenuation estimation from zero-offset VSP data using seismic interferometry. Geophysical Journal International, 204(2), 1288-1307.

Nakahara, H. (2006). A systematic study of theoretical relations between spatial correlation and Green's function in one-, two-and three-dimensional random scalar wavefields. Geophysical Journal International, 167(3), 1097-1105.

Paap, B., Verdel, A., Meekes, S., Steeghs, P., Vandeweijer, V., \& Neele, F. (2014). Four years of experience with a permanent seismic monitoring array at the Ketzin $\mathrm{CO} 2$ storage pilot site. Energy Procedia, 63, 4043-4050.

Panea, I., Draganov, D., Almagro Vidal, C., \& Mocanu, V. (2014). Retrieval of reflections from ambient noise recorded in the Mizil area, Romania. Geophysics, 79(3), Q31-Q42.

Ramírez, A. C., \& Weglein, A. B. (2009). Green's theorem as a comprehensive framework for data reconstruction, regularization, wavefield separation, seismic interferometry, and wavelet estimation: A tutorial. Geophysics, 74(6), W35-W62.

Rickett, J., \& Claerbout, J. (1996). Passive seismic imaging applied to synthetic data. Stanford Exploration Project, 92, 83-90.

Roux, P., Sabra, K. G., Gerstoft, P., Kuperman, W. A., \& Fehler, M. C. (2005). P-waves from cross-correlation of seismic noise. Geophysical Research Letters, 32(19).

Ruigrok, E., Campman, X., \& Wapenaar, K. (2011). Extraction of P-wave reflections from microseisms. Comptes Rendus Geoscience, 343(8-9), 512-525.

Sabra, K. G., Gerstoft, P., Roux, P., Kuperman, W. A., \& Fehler, M. C. (2005). Surface wave tomography from microseisms in Southern California. Geophysical Research Letters, 32(14).

Sager, K., Ermert, L., Boehm, C., \& Fichtner, A. (2018). Towards full waveform ambient noise inversion. Geophysical Journal International, 212(1), 566-590.

Sager, K., Boehm, C., Ermert, L., Krischer, L., \& Fichtner, A. (2020). Global-Scale FullWaveform Ambient Noise Inversion. Journal of Geophysical Research: Solid Earth, 125(4), e2019JB018644.

Schuster, G. T. (2001, June). Theory of daylight/interferometric imaging-tutorial. In 63rd EAGE conference \& exhibition (pp. cp-15). European Association of Geoscientists \& Engineers.

Schuster, G. T., Yu, J., Sheng, J., \& Rickett, J. (2004). Interferometric/daylight seismic imaging. Geophysical Journal International, 157(2), 838-852. 
Sens-Schönfelder, C., \& Wegler, U. (2006). Passive image interferometry and seasonal variations of seismic velocities at Merapi Volcano, Indonesia. Geophysical research letters, 33(21).

Shapiro, N. M., \& Campillo, M. (2004). Emergence of broadband Rayleigh waves from correlations of the ambient seismic noise. Geophysical Research Letters, 31(7).

Shapiro, N. M., Campillo, M., Stehly, L., \& Ritzwoller, M. H. (2005). High-resolution surface-wave tomography from ambient seismic noise. Science, 307(5715), 1615-1618.

Snieder, R. (2004). Extracting the Green's function from the correlation of coda waves: A derivation based on stationary phase. Physical Review E, 69(4), 046610.

Thorbecke, J. (2013). 2d finite-difference wavefield modelling. Book, 1-47.

Thorbecke, J. W., \& Draganov, D. (2011). Finite-difference modeling experiments for seismic interferometry. Geophysics, 76(6), H1-H18.

Tkalčić, H., Phạm, T. S., \& Wang, S. (2020). The Earth's coda correlation wavefield: Rise of the new paradigm and recent advances. Earth-Science Reviews, 103285.

Tonegawa, T., Nishida, K., Watanabe, T., \& Shiomi, K. (2009). Seismic interferometry of teleseicmic S-wave coda for retrieval of body waves: an application to the Philippine Sea slab underneath the Japanese Islands. Geophysical Journal International, 178(3), 15741586.

Tsuji, T., Ikeda, T., Johansen, T. A., \& Ruud, B. O. (2016). Using seismic noise derived from fluid injection well for continuous reservoir monitoring. Interpretation, 4(4), SQ1SQ11.

van Manen, D. J., Robertsson, J. O., \& Curtis, A. (2005). Modeling of wave propagation in inhomogeneous media. Physical Review Letters, 94(16), 164301.

Wang, B. L., Zhu, G. M., \& Gao, J. H. (2010). Joint interferometric imaging of walkaway VSP data. Applied Geophysics, 7(1), 41-48.

Wapenaar, K. (2004). Retrieving the elastodynamic Green's function of an arbitrary inhomogeneous medium by cross correlation. Physical review letters, 93(25), 254301.

Wapenaar, K., Fokkema, J., \& Snieder, R. (2005). Retrieving the Green's function in an open system by cross correlation: A comparison of approaches (L). The Journal of the Acoustical Society of America, 118(5), 2783-2786.

Wapenaar, K., \& Fokkema, J. (2006). Green's function representations for seismic interferometry. Geophysics, 71(4), SI33-SI46. 
Wapenaar, K., Thorbecke, J., \& Draganov, D. (2004). Relations between reflection and transmission responses of three-dimensional inhomogeneous media. Geophysical Journal International, 156(2), 179-194.

Weaver, R. L., \& Lobkis, O. I. (2001). Ultrasonics without a source: Thermal fluctuation correlations at MHz frequencies. Physical Review Letters, 87(13), 134301.

Willis, M. E., Lu, R., Campman, X., Nafi Toksöz, M., Zhang, Y., \& Hoop, M. V. D. (2006). A novel application of time-reversed acoustics: Salt-dome flank imaging using walkaway VSP surveys. Geophysics, 71(2), A7-A11.

Xiao, X., Zhou, M., \& Schuster, G. T. (2006). Salt-flank delineation by interferometric imaging of transmitted P-to S-waves. Geophysics, 71(4), SI197-SI207.

Xu, Z., Juhlin, C., Gudmundsson, O., Zhang, F., Yang, C., Kashubin, A., \& Lüth, S. (2012). Reconstruction of subsurface structure from ambient seismic noise: an example from Ketzin, Germany. Geophysical Journal International, 189(2), 1085-1102.

Yao, H., van Der Hilst, R. D., \& De Hoop, M. V. (2006). Surface-wave array tomography in SE Tibet from ambient seismic noise and two-station analysis-I. Phase velocity maps. Geophysical Journal International, 166(2), 732-744.

Zhan, Z., Ni, S., Helmberger, D. V., \& Clayton, R. W. (2010). Retrieval of Moho-reflected shear wave arrivals from ambient seismic noise. Geophysical Journal International, 182(1), 408-420.

Zhou, W., \& Paulssen, H. (2017). P and S velocity structure in the Groningen gas reservoir from noise interferometry. Geophysical Research Letters, 44(23), 11-785. 


\section{Appendix A) Zero-Offset VSP Cross-correlation MATLAB Code}

\% Zero-Offset VSP Cross-correlation Script

$\% \%$ Reading .segy file

[Data,SegyTraceHeaders,SegyHeader]=ReadSegy(filename)

xlabel('Trace Number');ylabel('Time (s)'

$\% \%$ Cross-correlation of master trace with the other traces

master_trc=Data(:,1);

for $\mathrm{i}=1$ :size(Data,2)

CORR_Trc(:,i)=xcorr(master_trc(:,1),Data(:,i));

end

$\%$ Writing .segy file as an output

WriteSegyStructure(filename,SegyHeader,SegyTraceHeaders,CORR_Trc(15001:30001,: ),'revision',1) 


\section{Appendix B) Zero-Offset VSP Processing on Seismic Unix}

$\# ! /$ bin/csh

set nrec $=98$

set tmax $=2.0$

sushw $<$ rejected.su key=scalel,gelev a $=-1,10600 \mathrm{~b}=0,200>$ rejected_geom.su

awk ' $\{$ print $\$ 1, \$ 2\}$ ' pickfile_excel.txt > pickfile_excel.txt.tmp

\# display timepick

suwind $<$ rejected_geom.su tmax=\$tmax $\mid$ suxwigb windowtitle="timepick" perc=99 $\backslash$ curve=pickfile_excel.txt.tmp npair $=\$$ nrec, 1 curvecolor $=$ red style $=v s p$ key $=$ gelev label1="owt(s)" label2=depth"(m)"\&

\# inject the timepick to data

\# prefer to inject in full wavefield, so the headers kept in subsequent process

awk '\{print $\$ 1 * 1000\}$ ' pickfile_excel.txt.tmp > pickfile_excel.txt.tmp2

$\mathrm{a} 2 \mathrm{~b}<$ pickfile_excel.txt.tmp2 $\mathrm{n} 1=1>$ rejected_fa.bin

b2a $<$ rejected_fa.bin $n 1=1>$ tmp.ascii

sushw $<$ rejected_geom.su infile=rejected_fa.bin key=tstat $>$ rejected_geom.su.pick 
\# check if headers are present

\#sugethw $<$ \$inp.pick key=tstat,tracf $\mid$ head $-\mathrm{n} 10$

\# f-k filtering

\# downgoing

sudipfilt $<$ rejected_geom. su.pick $\mathrm{dx}=20 \mathrm{dt}=0.008$ slopes $=-0.0005,-0.0002,0.0$

amps $=0,0,1>$ rejected_geom.su.pick.dn.fkfilt.su

\# upgoing

sudipfilt $<$ rejected_geom.su.pick $\mathrm{dx}=20 \mathrm{dt}=0.008$ slopes $=0.0,0.0002,0.0005 \mathrm{amps}=1,0,0$ $>$ rejected_geom.su.pick.up.fkfilt.su

suwind tmax $=\$ \operatorname{tmax}<$ rejected_geom.su.pick.dn.fkfilt.su $\mid$ suxwigb windowtitle="Dn after FK" perc $=99 \backslash$

curve=pickfile_excel.txt.tmp npair $=\$$ nrec, 1 curvecolor $=$ red style $=$ vsp key $=$ gelev label1="owt(s)" label2=depth"(m)"\&

suwind tmax $=\$ \operatorname{tmax}<$ rejected_geom.su.pick.up.fkfilt.su $\mid$ suxwigb windowtitle="Up after $\mathrm{FK} "$ perc $=99 \backslash$

curve $=$ pickfile_excel.txt.tmp npair $=\$$ nrec, 1 curvecolor $=$ red style $=\mathrm{vsp}$ key $=\mathrm{gelev}$ label1="owt(s)" label2=depth"(m)"\&

\# again, check if headers present after $\mathrm{f}-\mathrm{k}$

sugethw $<$ rejected_geom.su.pick.up.fkfilt.su key=tstat,tracf,gelev $\mid$ head -n 10

\# flattening the upgoing

awk '\{print $\$ 1 * 2, \$ 2\}$ ' pickfile_excel.txt.tmp > pickfile_excel.txt.twt.tmp2 
sustatic $<$ rejected_geom.su.pick.up.fkfilt.su hdrs=1 sign=-1 > rejected_geom.su.pick.up.fkfilt.flat.su

\#suwind tmax=\$tmax $<$ \$inp.pick.up.fkfilt.flat.su $\mid$ suxwigb windowtitle="input FK Flat" perc $=99 \backslash$

\# $\quad$ curve $=\$$ tt.twt.tmp2 npair $=\$$ nrec, 1 curvecolor=red key=gelev label1="twt(s)" label2=depth"(m)"\&

\# corridor stack

\# set parameter

\# corridor design, mute before Transit Time

\# add \$corridor window after transit time

\# last 5 traces stacked entirely, not as corridor

\# compare with display

set corridor $=0.1 \mathrm{\# S}$

set except_last_trace $=5$

(a) last_rec $=($ \$nrec - \$except_last_trace $)$

awk $-\mathrm{v}$ corridor $=\$$ corridor $-\mathrm{v}$ last_rec $=\$$ last_rec $\backslash$

'BEGIN $\{\mathrm{i}=1\}\{$ if $(\mathrm{i}<=$ last_rec $)\{$ print $((\$ 1 * 2)+$ corridor $), \$ 2\}$ else $\{$ print $9, \$ 2\}$ $\mathrm{i}++\}$ ' pickfile_excel.txt.tmp $\backslash$

| awk ' $\{$ printf "\%4f \%dln", \$1, \$2 \}'> pickfile_excel.txt.inside

awk ' $\{$ printf "\%4f \%d $/ n ", \$ 1 * 2, \$ 2\}$ ' pickfile_excel.txt.tmp > pickfile_excel.txt.outside 


\section{\# depth header}

awk ' \{print $\$ 2 * 10\}$ ' pickfile_excel.txt.tmp > xfile.tmp

$\mathrm{a} 2 \mathrm{~b}<$ xfile.tmp $\mathrm{n} 1=\$$ nrec $>$ xfile.bin

awk '\{print \$1\}' pickfile_excel.txt.inside > tfile.inside.tmp

$\mathrm{a} 2 \mathrm{~b}<$ tfile.inside.tmp $\mathrm{n} 1=\$$ nrec $>$ tfile.inside.bin

awk '\{print $\$ 1$ \}' pickfile_excel.txt.outside > tfile.outside.tmp

$\mathrm{a} 2 \mathrm{~b}<$ tfile.outside.tmp $\mathrm{n} 1=\$$ nrec $>$ tfile.outside. bin

\# display corridor window

\# mute before TT

sumute $<$ rejected_geom.su.pick.up.fkfilt.flat.su key $=$ gelev nmute $=\$$ nrec $x$ file $=x$ file.bin tfile $=$ tfile. outside. bin mode $=0 \backslash$

| suwind tmin $=0$ tmax $=\$ \operatorname{tmax} \mid$

| suxwigb perc=99.9 windowtitle="Flat Upgoing after FK (nodecon)" key=gelev $\backslash$

curve=pickfile_excel.txt.inside,pickfile_excel.txt.outside npair $=\$$ nrec, $\$$ nrec curvecolor=red label1='TWT (s)' label2='depth'\&

\section{\# mute between corridor and stack}

sumute $<$ rejected_geom.su.pick.up.fkfilt.flat.su key $=$ gelev nmute $=\$$ nrec $x$ file $=x$ file.bin tfile $=$ tfile.outside. bin mode $=0 \backslash$

| sumute $\mathrm{key}=\mathrm{gelev}$ nmute $=\$$ nrec $x$ file $=\mathrm{xfile}$. bin tfile $=$ tfile.inside.bin mode $=1 \backslash$

| sustack repeat $=10$ normpow $=1.0$ verbose $=1>$

rejected_geom.su.pick.up.fkfilt.flat.cstack.su 


\section{\# display corridor stack}

suwind $<$ rejected_geom.su.pick.up.fkfilt.flat.cstack.su tmin $=0$ tmax $=\$ \operatorname{tmax} \backslash$ | suxwigb perc $=99$ xbox $=610$ wbox $=200$ labell $=$ 'TWT $(\mathrm{s}){ }^{\prime}$ windowtitle='Corridor Stack'\&

\section{\# get surface seismic}

suwind $<$ stack_after_NMO.su key $=$ tracl $\min =100 \max =200$ tmin $=0$ tmax $=\$$ tmax $\mid$ suchw key $1=$ tracl key $2=$ tracl $a=-15>$ left.tmp.su

suwind $<$ stack_after_NMO.su key $=$ tracl $\min =200 \max =300$ tmin $=0$ tmax $=\$$ tmax $\mid$ suchw key $1=\operatorname{tracl}$ key $\overline{2}=\operatorname{tracl} \mathrm{a}=+15>$ right.tmp.su

suwind $<$ rejected_geom.su.pick.up.fkfilt.flat.cstack.su tmin $=0$ tmax $=\$$ tmax $\mid$ suchw keyl $=$ tracl key $2=$ tracl $a=+100>$ middle.cstack.tmp.su

\# combining surface seismic \& VSP

cat left.tmp.su middle.cstack.tmp.su right.tmp.su > splice.tmp.su

suximage $<$ splice.tmp.su perc $=99$ cmap $=$ rgb1 windowtitle $=$ 'Corridor Stack Spliced into Surface Seismic'\&

$$
\begin{aligned}
& \text { \# clean up } \\
& \text { \# rm *tmp* } \\
& \text { \# rm *bin* }
\end{aligned}
$$




\title{
11. Appendix C) Generating Synthetic Seismogram MATLAB Code
}

\author{
clc; \\ clear; \\ close all; \\ $\% \%$ \\ $\%$ Simple script to create a 1D synthetic seismogram by "convolving" a Ricker \\ $\%$ wavelet with a reflection coefficient series. Uses the function \\ $\%$ "ricker.m", written by Dan Lizarralde, to define the source wavelet. \\ $\%$ \\ $\%$ OUTPUT: Plots of the source function, and a three-panel plot showing: \\ $\% \quad$ (1) the velocity-depth function; \\ $\% \quad$ (2) the reflection coefficient series, and \\ $\% \quad$ (3) the synthetic seismogram \\ $\%$ \\ $\%$ \\ $\%$ INPUT: $\mathrm{dt}=$ time (seconds) interval of seismogram \\ $\% \quad$ thick $=$ vector containing thicknesses of each layer (Note that \\ $\% \quad$ these are thicknesses, NOT depths! \\ $\% \quad \mathrm{v}=$ vector containing velocity in each layer, plus one value for velocity \\ $\% \quad$ beneath the last boundary (e.g., if "thick" has 5 entries, then v must \\ $\%$ have 6 , etc.). \\ $\% \quad$ rho $=$ vector containing the density of each layer; the same number of \\ entries as $\mathrm{v}$. \\ $\% \quad \mathrm{fw}=$ center frequency of Ricker wavelet \\ clear all; close all; \\ $\mathrm{dt}=0.008$ \\ $\mathrm{fw}=24$;

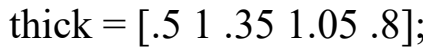 \\ $\%$ Thickness of layer above each reflector(s) \\ $\mathrm{v}=\left[\begin{array}{lllll}3.0 & 4.0 & 2.5 & 4.0 & 5.5\end{array}\right]$; \\ $\%$ Velocity above each reflector; last velocity is for \\ underlying half-space \\ rho $=\left[\begin{array}{lllll}1.0 & 1.4 & 1.5 & 1.8 & 2.2\end{array}\right] ; \quad \quad \%$ Density above each reflector; last density is for \\ underlying half-space \\ $\mathrm{Nt}=$ floor $(2 * \operatorname{sum}($ thick$) / \min (\mathrm{v}) / \mathrm{dt}) ; \quad \%$ Length of seismogram (\# of samples); using \\ minimum velocity ensure long enough trace \\ $\operatorname{Tmax}=(\mathrm{Nt}-1) * \mathrm{dt} ; \quad \%$ Maximum time of trace
}




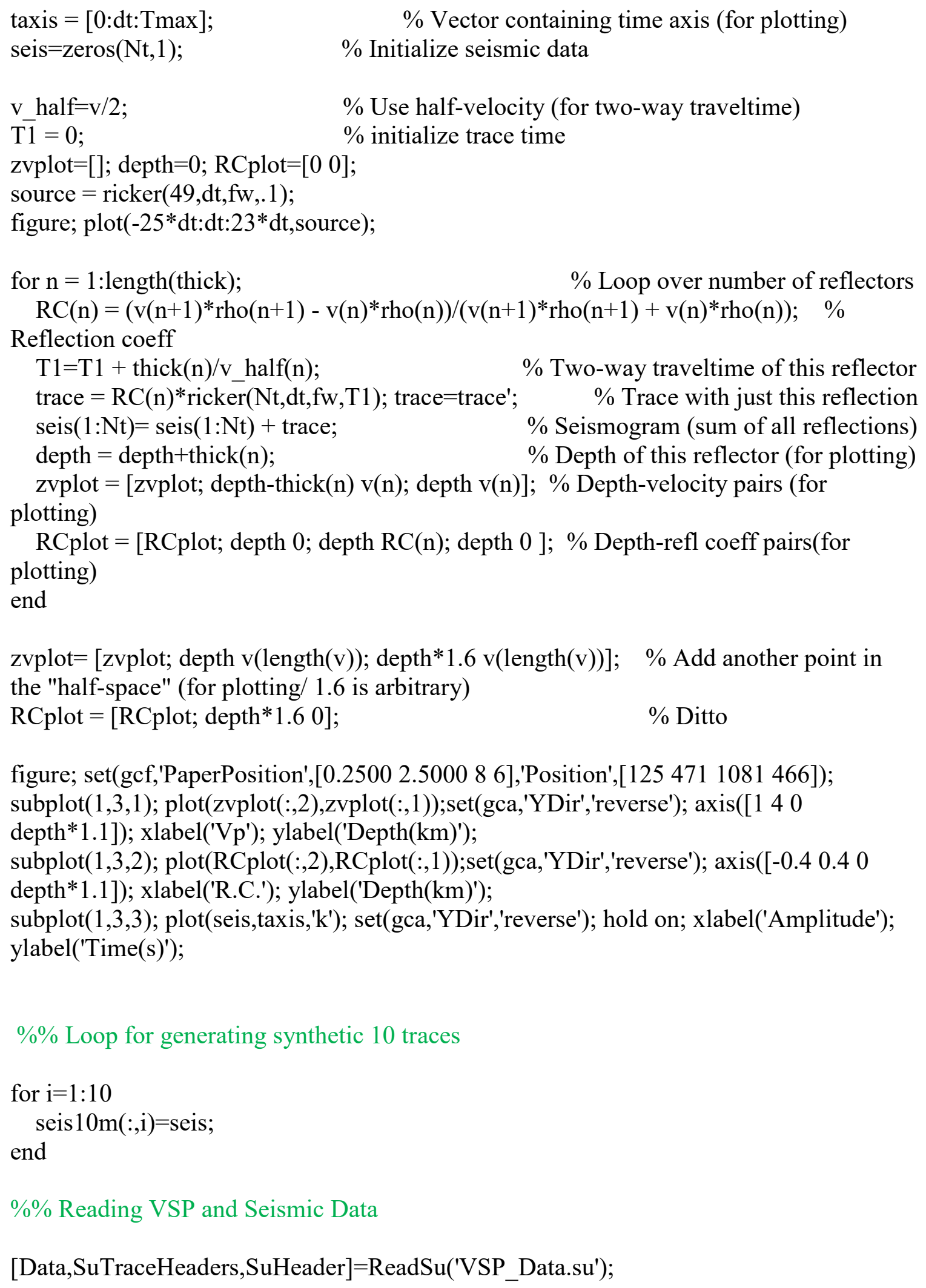


[Data2,SuTraceHeaders2,SuHeader2]=ReadSu('Seismic_Data.su');

$\% \%$ Plotting VSP-Seismic-Synthetic Seismogram

Figure

subplot $(1,3,1)$

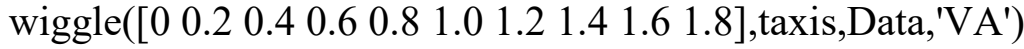

title('VSP SEISMOGRAM')

$\%$

subplot $(1,3,2)$

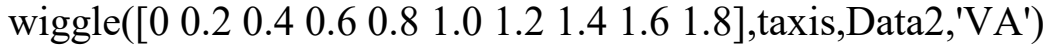

title('SEISMIC SEISMOGRAM')

$\%$

subplot $(1,3,3)$

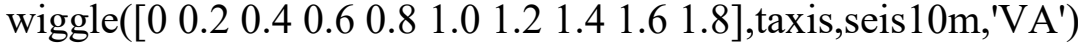

title('SYNTHETIC SEISMOGRAM')

$\% \%$ Generating Ricker Function

function $[\mathrm{S}]=\operatorname{ricker}(\mathrm{Nt}, \mathrm{dt}, \mathrm{fo}, \mathrm{t} 0)$

pi $=3.14159265$;

br $1=\left(\mathrm{pi}^{*} \mathrm{fo}\right)^{\wedge} 2$;

for it $=1: \mathrm{Nt}$

time $=(\mathrm{it}-1) * \mathrm{dt}$;

br $2=$ br $1 *(\text { t0 }- \text { time })^{\wedge} 2$;

$\mathrm{S}(\mathrm{it})=(1-2 * \mathrm{br} 2) * \exp (-1 * \mathrm{br} 2)$; end

end 